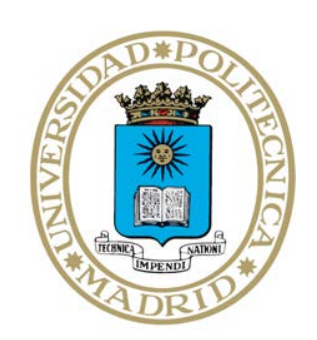

TECHNICAL UNIVERSITY OF MADRID

ESCUELA DE INGENIEROS DE

CAMINOS, CANALES Y PUERTOS

\title{
LONG-TERM OPTIMAL HYDROPOWER RESERVOIR OPERATION WITH MINIMUM FLOWS AND MAXIMUM RAMPING RATES
}

Thesis submitted for the degree of Doctor in Civil Engineering Systems

by

Ignacio Guisández González

Ingeniero de Caminos, Canales y Puertos (UPM)

Máster Universitario en Investigación en Ingeniería Eléctrica, Electrónica y Control Industrial (UNED)

July 2016 


\section{DEPARTMENT OF HYDRAULIC, ENERGY AND ENVIRONMENTAL ENGINEERING}

ESCUELA DE INGENIEROS DE CAMINOS, CANALES Y PUERTOS

\section{LONG-TERM OPTIMAL HYDROPOWER RESERVOIR OPERATION WITH MINIMUM FLOWS AND MAXIMUM RAMPING RATES}

Author: Ignacio Guisández González

Ingeniero de Caminos, Canales y Puertos (UPM)

Máster Universitario en Investigación en Ingeniería

Eléctrica, Electrónica y Control Industrial (UNED)

Supervisors: Juan Ignacio Pérez Díaz

Doctor Ingeniero de Caminos, Canales y Puertos (UPM) José Román Wilhelmi Ayza

Doctor Ingeniero de Caminos, Canales y Puertos (UPM)

July 2016 
This thesis was awarded in 2018 by both the Technical University of Madrid and the José Entrecanales Ibarra Foundation. The research that it contains was funded by the Spanish Ministry of Science and Innovation (CGL2009-14258), the Department of Hydraulic, Energy and Environmental Engineering of the Technical University of Madrid (Carlos González Cruz Scholarship), and SINTEF Energy Research (three-month research stay at Trondheim). 


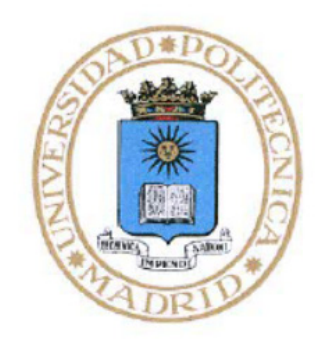

\section{UNIVERSIDAD POLITÉCNICA DE MADRID ESCUELA DE INGENIEROS DE CAMINOS, CANALES Y PUERTOS}

Departamento de Ingeniería Civil: Hidráulica, Energía y Medio Ambiente

Tribunal nombrado por el Magnífico y Excelentísimo Sr. Rector de la Universidad Politécnica de Madrid para juzgar la Tesis Doctoral
Presidente
D. Andrés Ramos Galán
Vocal
D. Michael M. Belsnes
Vocal
D. Edgardo D. Castronuovo
Vocal
D. Javier García González
Secretario
D. Luis Mediero Orduña

Calificación

Sobresaliente

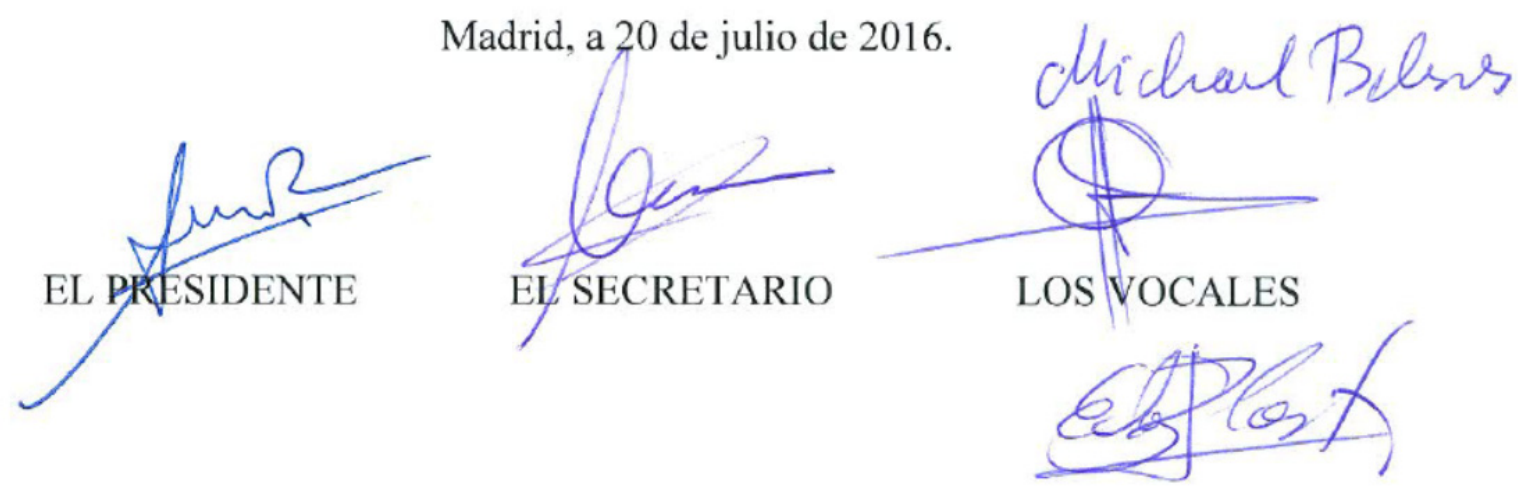

Madrid, a 20 de julio de 2016. 
a mis padres 


\section{Contents}

$\begin{array}{lr}\text { Abstract } & 1\end{array}$

$\begin{array}{ll}\text { Resumen } & 3\end{array}$

$\begin{array}{lr}\text { Coautores } & 5\end{array}$

$\begin{array}{ll}\text { 1. INTRODUCTION } & 7\end{array}$

1.1. Overview ........................... . . . 7

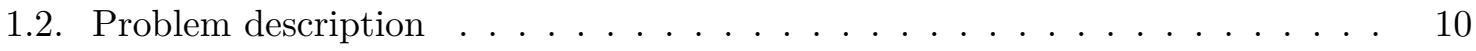

1.3. Scope and objectives . . . . . . . . . . . . . . . . . . . . . . . . . 12

1.4. Structure of the thesis . . . . . . . . . . . . . . . . . . . . 13

2. LITERATURE REVIEW $r \mid 15$

2.1. Decision support tools . . . . . . . . . . . . . . . . . . . . 15

2.1.1. Decision-making approaches . . . . . . . . . . . . . . . 15

2.1.2. Uncertainty treatments . . . . . . . . . . . . . . . . 16

2.1.3. Control approaches . . . . . . . . . . . . . 16

2.2. Optimisation techniques . . . . . . . . . . . . . . . . . . . 17

2.2.1. Linear programming . . . . . . . . . . . . . . . . 17

2.2.2. Dynamic programming . . . . . . . . . . . . . . . 20

2.3. Input variables . . . . . . . . . . . . . . . . . . . . . . . . 24

2.3.1. Markov chain theory . . . . . . . . . . . . . . . . . . 25

2.3.2. Water inflow ................. . . 26

2.3.3. Energy price . . . . . . . . . . . . . . . . . 27

2.3.4. Evaporation . . . . . . . . . . . . . . 27

2.4. Generation characteristics . . . . . . . . . . . . . . . . . . . 28

2.4.1. Performance curves . . . . . . . . . . . . . . . . . . . 28

2.4.2. Instantaneous load dispatch . . . . . . . . . . . . . . . . . . . . . 29

2.5. Hydro scheduling models with minimum flows and maximum ramping rates . 30

2.5.1. Linear programming models . . . . . . . . . . . . . . . . . . . . 30

2.5.2. Non-linear programming models . . . . . . . . . . . . . . . . 35

2.5.3. Dynamic programming models . . . . . . . . . . . . . 36

2.5.4. Other models . . . . . . . . . . . . . . . . . . . . . 37

2.5.5. Common absences of the cited models relevant for this work . . . . . . 38 
3. ASSESING THE LONG-TERM EFFECTS OF MINIMUM FLOWS AND MAXIMUM RAMPING RATES ON HYDROPEAKING

3.1. Assessment of the economic impact of environmental constraints on annual hydropower plant operation . . . . . . . . . . . . . . . . . . . 41

3.1.1. Methodology . . . . . . . . . . . . . . . . . . 41

3.1.2. Case study . . . . . . . . . . . . . . . . . . 45

3.1.3. Main results and discussion . . . . . . . . . . . . . . . 46

3.2. Effects of the maximum flow ramping rates on the long-term operation decisions of a hydropower plant . . . . . . . . . . . . . . . . . . 49

3.2.1. Methodology . . . . . . . . . . . . . . . . . . . 49

3.2 .2 . Case study . . . . . . . . . . . . . . . . . . 51

3.2.3. Main results and discussion . . . . . . . . . . . . . . 52

3.3. Approximate formulae for the assessment of the long-term economic impact of environmental constraints on hydropeaking . . . . . . . . . . . 53

3.3.1. Methodology . . . . . . . . . . . . . . . 53

3.3.2. Case studies . . . . . . . . . . . . . . . . 56

3.3.3. Main results and discussion . . . . . . . . . . . . . . 57

4. LONG-TERM OPTIMISATION MODELS FOR HYDRO SCHEDULING SUBJECT TO MINIMUM FLOWS AND MAXIMUM RAMPING RATES 63

4.1. Influence of the environmental constraints on the water and flow values . . . 63

4.1.1. Methodology . . . . . . . . . . . . . . . 68

4.1.2. Case study . . . . . . . . . . . . . . . . . . . 71

4.1.3. Main results and discussion . . . . . . . . . . . . . . . 72

4.2. Optimisation models for long-term hydro scheduling subject to environmental constraints $($ part I) . . . . . . . . . . . . . . . 80

4.2.1. Methodology . . . . . . . . . . . . . . . . 80

4.2.2. Case study . . . . . . . . . . . . . . . . . . . . 81

4.2.3. Main results and discussion . . . . . . . . . . . . . . . 82

4.3. Optimisation models for long-term hydro scheduling subject to environmental constraints $($ part II $) \ldots \ldots \ldots \ldots \ldots$

4.3.1. Methodology . . . . . . . . . . . . . . 86

4.3.2. Case study . . . . . . . . . . . . . . . . . . 87

4.3.3. Main results and discussion . . . . . . . . . . . . . 87

5. CONCLUSIONS $\quad 89$

5.1. Main contributions . . . . . . . . . . . . . . . . . 89

5.2. Main conclusions . . . . . . . . . . . . . . . . . . . 90

5.3. Future research directions . . . . . . . . . . . . . . . . . . . 91 
A. EQUATIONS OF THE OPTIMISATION MODELS 93

A.1. Equations of the dynamic programming algorithms . . . . . . . . . . . . . 93

A.1.1. Recursive equations . . . . . . . . . . . . . . . . 93

A.1.2. State transition equations . . . . . . . . . . . . . . . . . 94

A.1.3. Revenue estimation equations . . . . . . . . . . . . . . . . 94

A.1.4. Convergence criteria . . . . . . . . . . . . . . . . . 95

A.2. Equations of the linear programming algorithms . . . . . . . . . . 95

A.2.1. Objective functions . . . . . . . . . . . . . . . . 95

A.2.2. Constraints . . . . . . . . . . . . . . . . . . 97

B. MAIN DATA OF THE CASE STUDIES 107

$\begin{array}{ll}\text { References } & 129\end{array}$

$\begin{array}{ll}\text { Nomenclature } & 151\end{array}$ 



\section{List of Figures}

2.1. Classification of reservoir decision support tools. . . . . . . . . . . . 17

2.2. Types of linear programming. . . . . . . . . . . . . . . . 18

2.3. Types of dynamic programming. . . . . . . . . . . . . . . . 21

3.1. Flowchart of the solution strategy of the study 1 in each scenario. . . . . . . . 43

3.2. Coupling of the flows released by the plant between consecutive stages. . . . . 45

3.3. Annual losses according to $\varphi$ and $\rho$ of the plant I corresponding to the: (a) Average of the water years; b) Very wet water year; c) Wet water year; d) Normal water year; e) Dry water year; f) Very dry water year scenario. . . . . 47

3.4. Average annual losses of the plant I according to: (a) $\varphi ;$ (b) p. . . . . . . . 48

3.5. Histogram of the initial and final $\mathrm{F}$ of the subproblems of the best policies with $\rho>0$ obtained by mod2stu1. . . . . . . . . . . . . . . . 48

3.6. Flowchart of the solution strategy of the study 3 in each hydropower plant. . $\quad 54$

3.7. Calculated and predicted long-term economic impact in terms of: (a) $\varphi$ of the plant $\mathrm{A}$; (b) $\varphi$ of the plant B; (c) $\rho$ of the plant $A ;(d) \rho$ of the plant B. . . .

3.8. Long-term economic impact in terms of $\varphi$ and $\rho$ of the plant: (a) A calculated by the models; (b) A predicted by formula (3.3); (c) B calculated by the models; (d) B predicted by formula (3.3). . . . . . . . . . . . . 60

3.9. Power - Price-duration curves of the annual plants in terms of: (a) $\varphi$; (b) $\rho$. .

3.10. Energy generated by the annual plants in terms of price quartiles and: (a) $\varphi$;

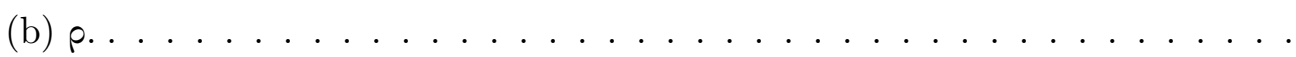

3.11. Variations relative to the problem without environmental constraints of the averages of the efficiency, net head, operating hours, spillage volume and startups and shut-downs of the annual plants in terms of: (a) $\varphi$; (b) p. . . . . . .

4.1. Examples of different $\rho \ldots \ldots \ldots \ldots$

4.2. (a) Historical weekly profiles (grey lines) and their average (black line) of the hourly energy prices of the first week of the water year; (b) Calculation of the weighting factors for disaggregation of the classes of the Markov chain of the energy price of the first week of the water year; (c) Hourly disaggregations of the three classes of the Markov chain of the energy price of the first week of the water year. . . . . . . . . . . . . . . . . . . 69

4.3. Average relative water values of the plant I in terms of: (a) $\varphi$; (b) p. . . . . 73 
4.4. Average water values of the plant I in terms of: (a) $\varphi$; (b) p. . . . . . . . 73

4.5. (a) Average relative water values of the plant I in terms of constant $\varphi$; (b) Average water values of the plant I in terms of constant $\varphi$; (c) Difference in average water values of the plant $I$ in terms of constant $\varphi . \ldots . . . . .$.

4.6. Average flow values of the plant I in terms of $\mathrm{p} \ldots \ldots \ldots . \ldots 75$

4.7. Water value level curves of the plant I of the scenario without environmental

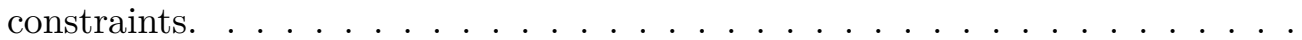

4.8. Water value level curves of the plant I with: (a) $\varphi=2 \%$; (b) $\rho=24 \mathrm{~h}$; (c) $\varphi=4 \%$; (d) $\rho=48 \mathrm{~h} ;$ (e) $\varphi=8 \%$; (f) $\rho=72 \mathrm{~h} . \ldots \ldots \ldots \ldots$

4.9. Average profiles of water value for five different, and constant through the year, weekly water inflows (Very dry=lowest cluster; Dry=second lowest cluster; Normal=medium cluster; Wet=second highest cluster; Very wet=highest

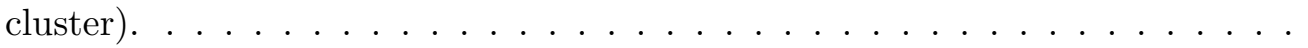

4.10. Flow value level curves of the plant I with: (a) $\rho=24 \mathrm{~h}$ at $\mathrm{V}=570 \mathrm{hm}^{3}$; (b) $\rho=24 \mathrm{~h}$ at $\mathrm{V}=123 \mathrm{hm}^{3}$; (c) $\rho=48 \mathrm{~h}$ at $\mathrm{V}=570 \mathrm{hm}^{3}$; (d) $\rho=48 \mathrm{~h}$ at $\mathrm{V}=123 \mathrm{hm}^{3}$; (e) $\rho=72 \mathrm{~h}$ at $\mathrm{V}=570 \mathrm{hm}^{3}$; (f) $\rho=72 \mathrm{~h}$ at $\mathrm{V}=123 \mathrm{hm}^{3}$. . . . . . . . . . .

4.11. Revenue of the plant B in the study 5 relative to $W V 00$ of: (a) $W V \varphi \rho$ in terms of $\varphi$; (b) both $W V \varphi \rho$ and $W \& F V \varphi \rho$ in terms of $\rho \ldots \ldots \ldots \ldots$

4.12. Long-term economic impacts of the plant B deterministically calculated in study 3 , those determined in study 5 through simulation considering $W V 00$, $W V \varphi \rho$ and $W \xi \xi V \varphi \rho \rho$, and those predicted by the formulae (3.1-3.2) in terms of: (a) $\varphi$; (b) $\rho \ldots \ldots \ldots \ldots \ldots \ldots \ldots \ldots \ldots \ldots \ldots$

4.13. Average power - price-duration curves across all simulations of the plant B in terms of: (a) $\varphi$ under $W V \varphi \rho$; (b) $\rho$ f under $W V 00 \ldots \ldots \ldots \ldots$

4.14. Average variations across all simulations relative to the problem without environmental constraints of the generated energy, operating hours, spillage volume and start-ups and shut-downs of the plant B in terms of: (a) $\varphi$ under $W V \varphi \rho$; (b) $\rho$ under $W V 00 \ldots \ldots \ldots \ldots \ldots \ldots \ldots \ldots \ldots \ldots$

4.15. Revenue of the plant B in the study 6 relative to $W V 00$ of: (a) $W V \varphi \rho$ in terms of $\varphi ;($ b) both $W V \varphi \rho$ and $W \mathscr{E} F V \varphi \rho$ in terms of $\rho \ldots \ldots \ldots \ldots$

5.1. Long-term effects of minimum flows and maximum ramping rates on hydropeaking. . . . . . . . . . . . . . . . . . . . .

B.1. Location of the plants used in the thesis (image taken from Google Earth ${ }^{\mathrm{TM}}$ ). 110

B.2. Water inflow scenarios of study 1: (a) Histogram and probability distribution;

(b) Hourly values for each scenario. . . . . . . . . . . . . . . . . . . . 110

B.3. Hourly and average weekly energy prices of studies 1 to $3 \ldots \ldots \ldots 111$ 
B.4. Maximum available flows of the bottom outlets and spillways of the plant I: (a) Right spillway; (b) Left spillway; (c) Bottom outlets; (d) Aggregate real values vs. Linear approximations. . . . . . . . . . . . . . . . . . .

B.5. Performance curves without hydraulic losses of the plant I: (a) Calculated values; (b) Linear concave approximation; (c) Linear non-concave approximation; (d) Calculated values vs. Linear concave approximation; (e) Calculated values vs. Linear non-concave approximation. . . . . . . . . . . . . . . . . .

B.6. Performance curves with hydraulic losses of the plant I: (a) Calculated values; (b) Linear concave approximation; (c) Calculated values vs. Linear concave

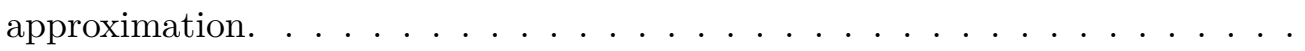

B.7. Performance curves without hydraulic losses of the plant II: (a) Calculated values; (b) Linear concave approximation; (c) Linear non-concave approximation; (d) Calculated values vs. Linear concave approximation; (e) Calculated values vs. Linear non-concave approximation. . . . . . . . . . . . . . . .

B.8. Performance curves without hydraulic losses of the plant III: (a) Calculated values; (b) Linear concave approximation; (c) Linear non-concave approximation; (d) Calculated values vs. Linear concave approximation; (e) Calculated values vs. Linear non-concave approximation. . . . . . . . . . . . . . . .

B.9. Performance curves without hydraulic losses of the plant IV: (a) Calculated values; (b) Linear concave approximation; (c) Linear non-concave approximation; (d) Calculated values vs. Linear concave approximation; (e) Calculated values vs. Linear non-concave approximation. . . . . . . . . . . . . . .

B.10.Performance curves without hydraulic losses of the plant V: (a) Calculated values; (b) Linear concave approximation; (c) Linear non-concave approximation; (d) Calculated values vs. Linear concave approximation; (e) Calculated values vs. Linear non-concave approximation. . . . . . . . . . . . . . .

B.11.Performance curves without hydraulic losses of the plant VI: (a) Calculated values; (b) Linear concave approximation; (c) Calculated values vs. Linear

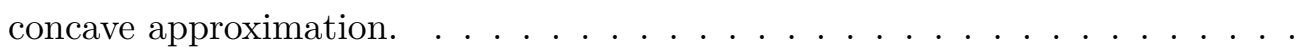

B.12.Performance curves without hydraulic losses of the plant VII: (a) Calculated values; (b) Linear concave approximation; (c) Calculated values vs. Linear concave approximation. . . . . . . . . . . . . . .

B.13.Performance curves without hydraulic losses of the plant VIII: (a) Calculated values; (b) Linear concave approximation; (c) Calculated values vs. Linear concave approximation. . . . . . . . . . . . .

B.14.Performance curves without hydraulic losses of the plant IX: (a) Calculated values; (b) Linear concave approximation; (c) Linear non-concave approximation; (d) Calculated values vs. Linear concave approximation; (e) Calculated values vs. Linear non-concave approximation. . . . . . . . . . . . 
B.15.Performance curves without hydraulic losses of the plant X: (a) Calculated values; (b) Linear concave approximation; (c) Linear non-concave approximation; (d) Calculated values vs. Linear concave approximation; (e) Calculated values vs. Linear non-concave approximation. . . . . . . . . . . . . . . . . . . 121

B.16.Performance curves without hydraulic losses of the plant A: (a) Calculated values; (b) Linear concave approximation; (c) Calculated values vs. Linear concave approximation. . . . . . . . . . . . . . . . . . 122

B.17.Performance curves without hydraulic losses of the plant B: (a) Calculated values; (b) Linear concave approximation; (c) Linear non-concave approximation; (d) Calculated values vs. Linear concave approximation; (e) Calculated values vs. Linear non-concave approximation. . . . . . . . . . . . . . . . . . 123

B.18.Head-storage curves of the plants: (a) I; (b) II; (c) III; (d) IV; (e) V; (f) VI. 124

B.19.Head-storage curves of the plants: (a) VII; (b) VIII; (c) IX; (d) X; (e) A; (f) B.125 B.20.Mean water inflow volumes of the plants: (a) I; (b) II; (c) III; (d) IV; (e) V; (f) VI. . . . . . . . . . . . . . . . . . . . . . . 126

B.21.Mean water inflow volumes of the plants: (a) VII; (b) VIII; (c) IX; (d) X; (e)

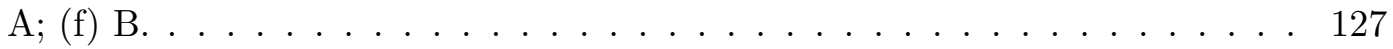




\section{List of Tables}

2.1. Advantages and disadvantages of linear and dynamic programmings in hydropower operation. . . . . . . . . . . . . . . . 24

2.2. Instantaneous load dispatch cases of a peak hydropower plant. . . . . . . . 29

3.1. Input variable scenarios and environmental constraints used in the study 1. . 45

3.2. Characteristic parameters of each model of the study 1. . . . . . . . . . 46

3.3. Input variable scenarios and environmental constraints used in the study 2. . 51

3.4. Characteristic parameters of the LP algorithms of the study 2. . . . . . . 52

3.5. Main values of the optimal policies of the plant I considered in the study 2. . 53

3.6. Input variable scenarios and environmental constraints used in the study 3. . $\quad 57$

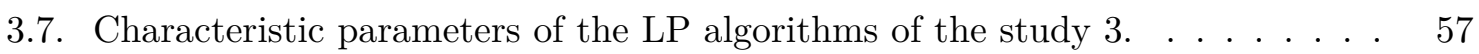

3.8. Main statistical parameters of the formulae and summary of the results obtained with the test plants. . . . . . . . . . . . . 58

4.1. Input variable scenarios and environmental constraints used in the study 4. . $\quad 72$

4.2. Characteristic parameters of the LP algorithms of the study 4 . . . . . . 72

4.3. Best correlation factors and their correlations with the flow values at $\mathrm{V}=347$ $h m^{3}$ of the plant I in terms of $\mathrm{p}$ and $\mathrm{F} \ldots \ldots \ldots \ldots 78 \ldots \ldots \ldots$

4.4. Input variable scenarios and environmental constraints used in the study 5. . 82

4.5. Characteristic parameters of the LP algorithms of the study 5. . . . . . . 82

4.6. Revenue of the plant $\mathrm{B}$ in the study 5 under the scenarios with combined $\varphi$ and $\rho$ relative to $W V 00$ of both $W V \varphi \rho$ and $W E \xi F V \varphi \rho \ldots \ldots \ldots$

4.7. Long-term economic impacts of the scenarios with combined $\varphi$ and $\rho$ on plant B determined in study 5 through simulation considering $W V 00, W V \varphi \rho$ and $W \& F V \varphi \rho$, and those predicted by the formula $(3.3) \ldots \ldots \ldots 5$

4.8. Differences between studies 5 and $6 \ldots \ldots \ldots \ldots$. . . . . . . . . 87

4.9. Revenue of the plant $B$ in the study 6 under the scenarios with combined $\varphi$ and $\rho$ relative to $W V 00$ of both $W V \varphi \rho$ and $W E F V \varphi \rho \ldots \ldots \ldots$

B.1. Main design parameters of the plant I used in the studies 1 and 2. . . . . 108

B.2. Main design parameters of the plant I used in the study 4 and of the plant B

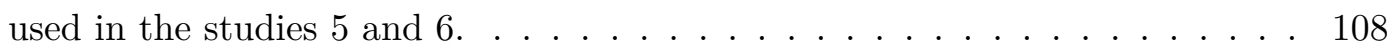

B.3. Main design parameters of the plants used in the study $3 . \ldots$. . . . . . . 109 



\section{Abstract}

This thesis studies the long-term operation of price-taker peak hydropower plants associated to reservoirs and subject to minimum flows and maximum ramping rates that sell energy in day-ahead electricity markets. The thesis is organised in five chapters and two appendixes.

The first chapter is an introduction of the above-mentioned issue. It aims to provide both an overview as well as a mathematical description of the addressed problem and define the scope and objectives of the thesis.

The second chapter shows a review of the literature related to the main topics tackled in the thesis, such as the principal approaches of reservoir decision support tools, the optimisation techniques most used in hydro scheduling, the main procedures for the characterisation of the involved random inputs and the methods most employed to estimate the generation characteristic of a peak hydropower plant. Furthermore, the chapter also presents a brief description of the hydro scheduling models that have considered minimum flows and maximum ramping rates jointly.

The third and the fourth chapters are devoted to the achievement of the thesis objectives and are divided in several studies. Among the main contributions cointaned in these studies can be found different long-term optimisation models for hydropeaking subject to minimum flows and maximum ramping rates, several sensitivity analyses of the long-term effects of these constraints on certain economical and operational aspects of a peak hydropower plant, a set of formulae for the approximate assessment of the long-term economic impact caused by these constraints on this type of plants, and the introduction of a new concept in hydro scheduling: flow value.

The fith chapter sets out the conclusions of the thesis which can be summarised as follows. On the one hand, the presence of minimum flows in hydropeaking increases the spillage volume and the water value, whereas decreases the generated energy, the number of start-ups and shut-downs of the hydro units, the plant capability for price tracking and the revenue. On the other hand, the presence of maximum ramping rates, in turn, increases the number of plant operating hours, the spillage volume, and, in the driest weeks, the flow value, whereas decreases the number of start-ups and shut-downs of the hydro units, the plant capability for price tracking, the revenue, the water value, and, in the wettest weeks, the flow value.

The appendix A contains the equations involved in the developed optimisation models and the appendix B provides a summary of the main data of the case studies considered in the third and fourth chapters. Finally, both the cited references and the applied nomenclature can be found at the end of the thesis. 



\section{Resumen}

Esta tesis estudia la operación a largo plazo de plantas hidroeléctricas asociadas a embalses sujetas a caudales mínimos y rampas máximas que participan en mercados eléctricos diarios como tomadoras de precios. La tesis se compone de cinco capítulos y dos apéndices.

El primer capítulo es una introducción de la cuestión mencionada. Proporciona una visión panorámica, así como una descripción matemática del problema abordado y define el alcance y los objetivos de la tesis.

El segundo capítulo muestra una revisión de la literatura relacionada con los principales temas afrontados en la tesis, tales como los principales enfoques de herramientas de ayuda a la toma de decisión en embalses, las técnicas de optimización más utilizados en programación hidroeléctrica, los principales procedimientos para la caracterización de las variables aleatorias de entrada implicadas y los métodos más empleados para estimar la característica de generación de una central hidroeléctrica de puntas. Además, el capítulo también presenta una breve descripción de los modelos de programación hidroeléctricos que han considerado conjuntamente caudales mínimos y rampas máximas.

Los capítulos tercero y cuarto están dedicados a la consecución de los objetivos de la tesis y se componen de varios estudios. Entre las principales aportaciones de estos estudios se pueden encontrar diferentes modelos de optimización a largo plazo para operación hidroeléctrica de puntas sujetos a caudales mínimos y rampas máximas, varios análisis de sensibilidad de los efectos a largo plazo de estas restricciones sobre ciertos aspectos económicos y operativos de una planta hidroeléctrica de puntas, un conjunto de fórmulas para la evaluación aproximada del impacto económico a largo plazo causado por dichas restricciones en este tipo de plantas, y la introducción de un nuevo concepto en programación hidroeléctrica: valor del caudal.

El quinto capítulo expone las conclusiones de la tesis que se pueden resumir de la siguiente manera. Por un lado, la presencia de caudales mínimos en la operación hidroeléctrica de puntas aumenta el volumen de vertido y el valor del agua, mientras que disminuye la energía generada, el número de arranques y paradas de los grupos hidroeléctricos, la capacidad de la planta para el seguimiento de los precios y los ingresos. Por otro lado, la presencia de rampas máximas, a su vez, aumenta el número de horas de funcionamiento de la planta, el volumen de vertido y, en las semanas más secas, el valor del caudal, mientras que disminuye el número de arranques y paradas de los grupos hidroeléctricos, la capacidad de la planta para el seguimiento de los precios, los ingresos, el valor del agua, y, en las semanas más húmedas, el valor del caudal.

El apéndice A contiene las ecuaciones que intervienen en los modelos de optimización desar- 
rollados y el apéndice B ofrece un resumen de los principales datos de los casos considerados en los estudios de los capítulos tercero y cuarto. Finalmente, tanto las referencias citadas como la nomenclatura aplicada se pueden encontrar al final de la tesis. 


\section{Coautores}

No recuerdo cuando fue la primera vez que me imaginé escribiendo estas líneas, pero de lo que si me acuerdo es que, ya entonces, intuí que agradecer sería muy poco en comparación con la ayuda recibida. Y así ha sido. De ahí la elección del encabezamiento superior, pues con él trato no sólo de dar las gracias, sino de compartir las pequeñas luces que contiene esta tesis.

Me resulta ineludible comenzar por los principales coautores de este documento: sus directores. Los profesores J.I. Pérez y J.R. Wilhelmi han sido el genial, en todas sus acepciones, motor de este trabajo siendo yo tan sólo su combustible, en muchas ocasiones para desgracia de ellos, no refinado. Son literalmente maravillosos y no creo que nunca pueda agradecerles todo lo que han hecho por mí.

Afortunada y generosamente, otras muchas personas han colaborado a la realización de este trabajo con su experiencia, consejo e incluso con sus preguntas. De entre todas ellas cabe destacar, por orden alfabético, a: A. Granados, A. Helseth, A. Rossi, D. García, D. Santillán, D. Valigi, E.E. Rodríguez, F.J. Guisández, G. Doorman, H.I. Skjelbred, J.A. Sánchez, J.I. Sarasúa, K.D.W. Nandalal, L. Garrote, L. Mediero, M. Chazarra, M.T. Guisández, R. Millán, S.P. Bianucci, S. Sañudo, y W. Dragoni.

Concluyo aquí mi tesis, en la festividad de Santo Domingo de la Calzada, y a menos de dos días de casarme con la mujer de mi vida, dando gracias a Dios por todo.

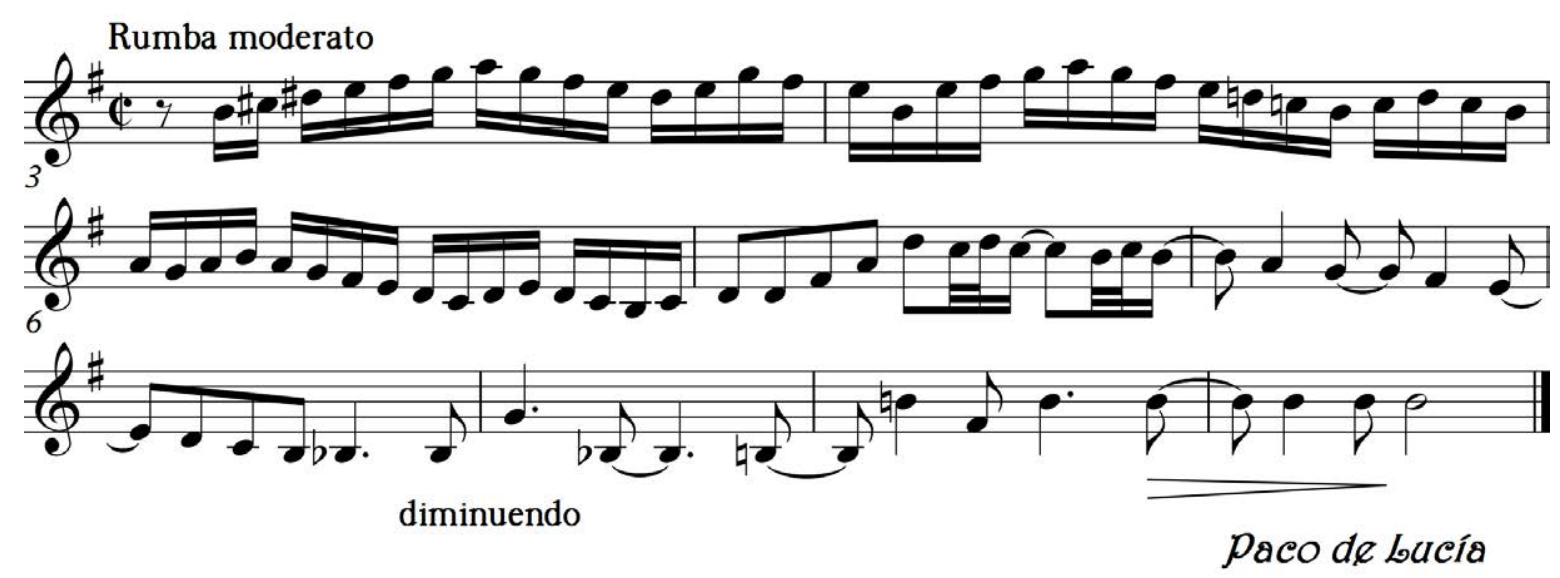





\section{INTRODUCTION}

This chapter aims to provide both an overview as well as a mathematical description of the addressed problem and define the objectives and structure of the thesis.

\subsection{Overview}

Energy is essential to economic and social development, to improve quality of life and it must be produced in a sustainable way [UN (1992)]. Electricity is the most versatile form of energy [Perrin (1971)], but nowadays cannot be economically stored [Uritskaya and Uritsky (2015)]. Thus, electricity must be produced at the moment of consumption and, for the sake of sustainability, it is increasingly generated by new renewable energy sources [BP (2016)], which are usually characterised by being weather-driven and non-dispatchable [Kirkham et al. (1981)].

In order to cope with the complexity of generating systems, maintaining high reliability and low cost, electric systems in many countries all over the world have been moved from a regulated to a liberalised competitive environments [Nogales et al. (2002)]. The resulting electricity markets operate, usually on a daily basis, assigning hourly energy prices which are signals that mirror the variations of cost of the energy supplied to the electric system [Schweppe et al. (1980)]; these variations result in avoided costs, reduction in $\mathrm{CO}_{2}$ emissions, etc [Pérez-Díaz and Wilhelmi (2010)]. The operation of these markets is developed in several stages of transaction [Smeers (2008)]: day-ahead, intra-day and real-time electricity markets.

In this context, it is worth highlighting one of the main advantages of hydroelectricity, that is, its ability to change the generated power quickly; additionally no significant losses are faced when at a standstill, except those caused by spillage, evaporation or seepage (depending on the type of plant and location). This feature allows hydroelectricity to meet hours with the highest energy consumption (peak hours) at minimum cost [Weedy (1972)] and, as a consequence, to play a crucial role in the integration of new intermittent renewable energy sources [World Water Council (2003)]. This activity is usually carried out by adapting its production to the energy price profile (hydropeaking) [Pérez-Díaz and Wilhelmi (2010)].

Hydroelectricy has the highest efficiency, flexibility and reliability of any source of electricity [Egré and Milewski (2002)] and therefore has become the fourth source of primary energy in the world and the first one among renewable energies [BP (2016)]. However, despite its renewable nature, several investigations indicate that hydropower plants can yield undesirable effects on the ecosystems where are located [Cushman (1985); Eckberg (1986); Pelc and 
Fujita (2002); Trussart et al. (2002)]. This is the motive why many countries have developed specific policies to reduce environmental impacts caused by this power generation technology [ESHA (2012)] and, as a result of these initiatives, the number of constraints to which hydro scheduling is subject has increased considerably [Kosnik (2010)].

A hydropower plant is a facility whose purpose is to convert part of the energy of water (kinetic, potential or pressure) into available electrical energy. There are many types of hydropower plants [Raabe (1985)], differing greatly from each other in their characteristics and modes of operation. This thesis is focused on the typology of peak hydropower plants associated to reservoirs since it has the greatest installed capacity in Europe [Lehner et al. (2005)].

The power generated by a peak hydropower plant depends on the efficiency of the above named conversion, on the mass of water flowing through each of its turbines per unit time and on the net head. The net head is the energy height at the turbine inlet referred to tailwater level [Warnick (1984)]. The mathematical representation of the relationship among power, flow and net head in a plant is called, i.a., generation characteristic [Pereira (1985)].

The operation of this type of plants is usually subject to technical, strategic and operational constraints [Pérez-Díaz and Wilhelmi (2010)]. Technical constraints habitually denote those restrictions derived from certain inherent properties of the plant generation equipment and hydraulic system as, e.g., the maximum and minimum flow through the turbines [Little (1955)]. Strategic constraints, in turn, consider commonly the tracking of longer term guidelines such as, for instance, water value curves [Boshier et al. (1983)]. Operational constraints can be due to several reasons such as, e.g., the existence of other priority uses in the reservoir [Kumar and Baliarsingh (2003)].

Environmental constraints may be sorted within the group of operational constraints [Edwards et al. (1999)]. There are various types of environmental constraints but the most common, and therefore the only ones considered in this thesis, are minimum flows $(\varphi)$, which force minimum values of water release (F) [EU (2007)], and maximum ramping rates ( $\rho)$, which impose maximum rates of change of F [Patten et al. (2001)].

Unfortunately, these environmental constraints may cause several negative effects on the operation of a peak hydropower plant. Perhaps the most important are, on the one hand, that $\varphi$ limit the ability to store water in the reservoir for use in peak hours and, on the other hand, that $\rho$ limit its ability to change power output levels [Harpman (1999)]; that is, these ramps introduce some kind of inertia in the plant operation.

The immediate consequences of these effects are the reduction in the above-mentioned ability to follow the load variations as well as in the contribution of the plant to the system operation [Harpman (1999)]. This contribution (ancillary services [Eurelectric (2003)]) is often provided by hydropower plants [Perekhodtsev and Lave (2005)].

The subsequent consequences are economic losses [Harpman (1999)], whose quantification is important to assess the implementation of new environmental regimes [Paredes-Arquiola et al. (2011)] and in relicensing negotiations [Rheinheimer et al. (2013)], as well as more 
operational difficulties for hydropower producers [Farhat and El-Hawary (2009)]. These last consequences are the main motivation of this thesis.

The aim of planning in any activity is to take into account the future consequences of present actions in order to reach a favourable future [Martino (1993)]. From the hydropower producer's point of view, as in any other company, this favourable future is usually that which maximises revenue. Accomplishing this goal is a difficult problem due, in addition to the said issues, to the random nature of the variables involved in hydropower operation such as water inflow, energy demand, wind generation, temperature and network contingencies [Forsund (2007)].

Water inflow affects the stored volume of water in the reservoir (V) which in turn influences the net head of the plant [Little (1955)]. Energy demand influences the energy price [Sauter and Lobashov (2011)]. Wind generation also affects the energy price, if the amount of installed wind power is significant, but it does in reverse direction, the higher the wind generation, the lower the energy price [Jensen and Skytte (2003)]. Temperature influences the energy price too; this influence is usually direct with high temperature values [Knittel and Roberts (2005)] and inverse with low ones [Koreneff et al. (1998)]. Furthermore, high values of both wind speed and temperature contribute to the increase in evaporation and, therefore, to the decrease in V and the net head [Harbeck (1962)]. Network contingencies are related to any problem in the electrical system able to affect externally the hydro operation, e.g.: transformer outages, transmission lines outages caused by storms, etc. [Forsund (2007)].

The key question in hydropower operation is to decide when to produce energy, given $\mathrm{V}$ and the available information about the future [Forsund (2007)]. Many decision support tools have been proposed during past decades to help to answer this question in real-time [Koutsoyiannis et al. (2002)], although very few under environmental constraints [Smith et al. (2007)], especially $\rho$ [Rheinheimer et al. (2013)] and more particularly in a long-term planning period (usually one year [Zhao et al. (2010)]).

Real-time in this context usually concerns the optimal operation of an existing reservoir system [Yeh (1985)] in which a complex [Guise and Flinn (1970)], and mostly specific [Howard (2006)], set of objectives has to be generally considered. This is due to the fact that each plant has its own production process and these processes depending on each other within each basin [Keppo (2002)]. In addition, from the point of view of the power market, the decision to produce is also conditioned by the installed capacity of each system: if its capacity is large enough to influence energy price, the system is a price-maker, otherwise a price-taker [Kelman et al. (2001)].

In conclusion, the problem of hydropeaking operation is hard in itself and even more if environmental criteria are considered. As a matter of fact, despite the large number of hydroelectric decision support tools developed for this purpose, this aspect has been rarely considered in great detail in the literature and even less in a long-term period. 


\subsection{Problem description}

The problem considered consists of studying the long-term optimal operation of hydropower reservoirs subject to $\varphi$ and $\rho$ acting as price-takers within liberalised electricity markets.

Optimisation is the science of finding the best solutions to certain mathematical problems [Fletcher (2000)]. Mathematically speaking, it deals with the minimisation or maximisation of a function (objective function), which represents the desired objective, subject to constraints on its variables [Nocedal and Wright (2006)]. In the case of the operation of a hydropower system, the best solution generally consists in maximising the value of the energy generated by the system over the time of study (planning period) plus the expected future revenue from $\mathrm{V}$ (water value) at the end of that time (time horizon) [Grygier and Stedinger (1985)].

Hydro scheduling is a multistage optimisation problem [Little (1955)]. In this type of problems, the state of the system at any stage is represented by a set of quantities (state variables). At each stage, a set of decisions (decision variables) has to be taken in order to lead the system to some feasible state at the beginning of the next stage. This transition is given by an equation (state transition equation) that takes into account the state at the beginning of the stage, the decisions made along the stage (time step) and the environment (input variables) that surround and affect the system during the stage [Bellman (1954)].

The evolution of the input variables along the planning period can be considered under two different approaches [Bellman (1954)]: known, whereby the initial state and the decisions uniquely determine the final state (deterministic approach), or unknown, so the initial state and the decisions determines a probability distribution of final states (stochastic approach).

Both approaches have drawbacks relative to each other. On the one hand, the deterministic approach provides in general worse results than the stochastic one [Wallace and Fleten (2003)], and on the other hand, the computational burden corresponding to the stochastic approach is much higher than that associated to the deterministic one [Zambelli et al. (2006)]. The choice between these approaches may be conditioned by any of these certainties: that of forecasts or that, which may be called, of system's equivalence.

The uncertainty of a forecast has sometimes been neglected when the planning period is short [Bensalem et al. (2007); Nandalal and Bogardi (2007)], e.g., a day [Keckler and Larson (1968)] or a week [Eschenbach et al. (2001)]. Deterministic approach may also be recommended if the system is certainty equivalent [Simon (1956)]. This is ensured when system output is measured by a quadratic function, the system dynamic is linear, there are no inequality constraints and input variables are independent and normally distributed [Philbrick and Kitanidis (1999)].

Unfortunately, hydro scheduling is a type of problem that is characterised by being discrete, non-concave and non-linear [Chang et al. (2001)]. Discreteness is due to the turbine generator set (hydro unit) on/off status (operating state). Non-concavity arises in some parts of the generation characteristic below its best efficiency points. Non-linearity is caused by the nature of the generation characteristic of the hydro units. 
Despite these features, the deterministic approach can provide both insight into the optimal behaviour of the hydropower plants [Soares and Carneiro (1991)] and very good results in those hydro systems whose outcomes can be approximated by a quadratic functions and, provided that, whose reservoir levels are kept at or near the optimum hydraulic heads, whose storage volume capacities cannot significantly restrict normal operations and whose inputs correspond to normal conditions [Karamouz and Houck (1987); Philbrick and Kitanidis (1999); Zambelli et al. (2006); Ratnayake and Harboe (2007); Ventura and Martinez (2011)].

The most common aspects to be defined in order to address hydro scheduling have been found to be some or all of the following [Yakowitz (1982); Yeh (1985); Wallace and Fleten (2003); Labadie (2004); Nandalal and Bogardi (2007); Iliadis et al. (2008); Gjelsvik et al. (2010); Fayaed et al. (2013)]:

- State transition equation: mass balance equation which represents the conservation of mass throughout the system.

- Planning period: from one day or one week (short-term) to a few months (mid-term) or one year or more (long-term).

- Time step: from one hour or one day (short-term) to a couple of weeks or one month (mid-term).

- Decision variables: released volume of each reservoir during each stage, or state to be reached at the beginning or the end of each stage.

- Input variables: water inflow into each reservoir, evaporation on each reservoir, energy demand or, lately, energy price.

- State variables: $\mathrm{V}$ in each reservoir at the beginning of each stage and also, under stochastic approach, previous or current stage's water inflow into each reservoir and its forecast for the next stage (if and only if it has not been selected as input variable), previous or current stage's energy demand or previous or, lately, current stage's mean energy price (if and only if neither of them have been selected as input variable).

In the perspective of real-time operation, a hydro scheduling problem should be solved by an optimisation algorithm on the basis of forecast information. As forecasts deteriorate with time and decisions based on the best possible information are necessary, the problem is usually approached by means of various models with several planning periods (short-, mid- and/or long-term), and different levels of detail, in such a manner that the shorter the planning period, the higher the level of detail [Yeh (1985); Labadie (2004)].

These models are hierarchically related as follows: outputs from one model are used as inputs into the next lower level model of shorter planning period, iterating and updating whenever new forecasts become available [Yeh (1985)]. This structure allows to reduce the computational burden and therefore the time necessary to obtain a solution [Yeh (2010)]. 
The process begins with the long- or mid-term model in order to determine the water value at the end of each stage along the planning period, which depends on future system development [Doorman (2009)]. Therefore, the stochasticity of the inputs should be considered in some manner but, due to these uncertainties and the associated computational cost, the generation characteristic at each plant is not usually defined with high accuracy. Inputs should be updated at each stage [Yeh (1985)] if there are new mid- or long-term forecasts but, if there are not, it is a good practice to run this model in an off-line phase considering the historical data of the inputs [Takeuchi and Moreau (1974); Braga Jr et al. (1991); Pritchard et al. (2005); Castelletti et al. (2008)].

Next, the process usually continues, until the end of the planning period, running in realtime the short-term model to provide hourly water releases of every plant [Howard (2006)]. This model, which should also be updated at each stage, is more accurate, its uncertainty level and computational burden are lower [Yeh (1985)], and its benefit sensitivity is higher than in the mid- or long-term models [Karamouz et al. (2005)].

According to the above, the water value plays an important role between the long- or mid- and the short-term models [Fosso and Belsnes (2004)] and, in this context, it can be understood as an opportunity benefit. Its magnitude is function of $\mathrm{V}$ and the time of the year [Stage and Larsson (1961)] (usually monotonically decreasing with V and with the amount of water inflow [Gebrekiros et al. (2013)] because of the increasing risk of spill [Perera (1969)]) and its performance is not optimal for a randomly given limited water inflow series [Doorman (2009)].

\subsection{Scope and objectives}

The thesis is focused on long-term optimal operation of peak hydropower plants associated to reservoirs, operating as price-takers within a liberalised day-ahead electricity market, and subject to $\varphi$ and $\rho$. In order to define more precisely the scope of the study, it should be mentioned that the objectives addressed in this thesis are oriented to the operation of single plants located at the heads of their basins.

The objectives of this thesis are the following:

1. To develop one or more long-term optimisation models for a real-time decision support tool of a plant subject to $\varphi$ and $\rho$.

2. To study the sensitivity of the water value of an existing plant to $\varphi$ and $\rho$.

3. To obtain one or more analytical expressions providing a rough estimate of the economic impact that $\varphi$ and $\rho$ would cause to the operation of a plant of given characteristics.

4. To contribute to the knowledge about the effects of $\varphi$ and $\rho$ on the operation of a plant. 


\subsection{Structure of the thesis}

Besides this introduction, the thesis is organised in four chapters and two appendixes as follows: chapter 2 reviews the key aspects of the literature related to the thesis, chapter 3 and chapter 4 deal with the achievement of the above-mentioned objectives, chapter 5 summarises the findings of the thesis and proposes future research avenues, appendix A contains the equations involved in the developed optimisation models and appendix B presents the main data of the considered case studies. Finally, both the used references and the applied nomenclature can be found at the end of the thesis. 



\section{LITERATURE REVIEW}

This chapter shows a review of the literature related to the main topics tackled in the thesis. It begins describing the principal approaches of reservoir decision support tools under which peak hydropower plants have also been managed. Next, the optimisation techniques most used in hydro scheduling are commented. Then, the main procedures used for the characterisation of the involved random inputs are presented. After, the methods most employed to estimate the generation characteristic of a peak hydropower plant are displayed. Finally, the chapter concludes with a brief description of the hydro scheduling models that have considered $\varphi$ and $\rho$ jointly.

\subsection{Decision support tools}

A decision support tool is any implement that is applied to a decision support process [Kapelan et al. (2005)], whose mission is to calculate and manage the attendant risks of potential decisions involved in the process [Buchanan and O'Connell (2006)].

There have been appeared a huge number of decision support tools in the field of reservoir system management during the last decades [Koutsoyiannis et al. (2002)]. These are often classified according to one or more of the following three aspects (Fig. 2.1), which here have been termed: decision-making approaches, uncertainty treatments and control approaches.

\subsubsection{Decision-making approaches}

The distinction of three main basic decision-making approaches of a decision support tool is widespread in the literature. These are simulation, optimisation and combined optimisationsimulation decision-making approaches. In what follows, the more important aspects of these approaches will be briefly discussed [Yeh (1985); Wurbs (1993); Labadie (2004); Loucks and van Beek (2005); Rani and Moreira (2010); Fayaed et al. (2013)].

The simulation approach consists in modelling a system in an accurate way and then, for each set of input data, obtaining a response. These input data include decision rules, thus a simulation model helps to answer what if questions concerning the performance of different operational policies and determine which is the best of all them.

The optimisation approach (as discussed in sec. 1.2) provides the best policy from a usually simpler model of the system in one or more scenarios. This policy is the set of decisions that optimise one or more objective functions subject to a set of constraints. 
Whereas simulation tools are restricted to foreseeing system performance for the evaluated scenarios, optimisation tools automatically search for the best response of the simplified model. The time spent to achieve this response it can be considerably less than that required by simulation.

The combined optimisation-simulation approach takes sequentially the best of both techniques. First, a suboptimal policy is obtained by means of an optimisation model. And then, this policy is simulated in order to furnish more precise evaluations of the system performance associated with the obtained decisions.

\subsubsection{Uncertainty treatments}

Uncertainty can be treated in two ways within decision support tools: implicit or explicit stochastic treatments. Their main features are presented below [Labadie (2004); Rani and Moreira (2010); Fayaed et al. (2013)].

The implicit treatment is characterised by the use, as input data, of series of historical or synthetically generated values of the random input variables, or equally likely sequences. In this option, the stochastic aspects of the problem are implicitly included and the resulting policy is obtained by means of regression analysis.

The explicit treatment works directly through the probability distributions of the random input variables by taking these functions as input data. The resultant policy is determined automatically by this approach.

Both treatments have drawbacks. In the implicit case, regression analysis can prove in reduced values of the correlations that would invalidate the obtained operating rules, and attempting to infer policies from other methods can need extensive trial and error processes with little general applicability. The explicit case, in turn, is more computationally challenging.

\subsubsection{Control approaches}

In its role of control tool, decision support tools may be structured in two ways: open- or closed-loop control approaches. Their short descriptions are as follows [Bras et al. (1983); Golnaraghi and Kuo (2003)].

In the open-loop approach the values of the decision variables throughout a planning period are chosen at the beginning of that period, regardless of system evolution.

In the closed-loop approach the decision support tool includes feedback from the system state and, thus, every decision is made according to the current state of the system, even the updated forecasts of the input variables.

The first approach is easier to implement and faster to execute but does not consider the system evolution. The second one is obviously more difficult to develop and slower to run but it includes feedback and therefore the results are more realistic. 


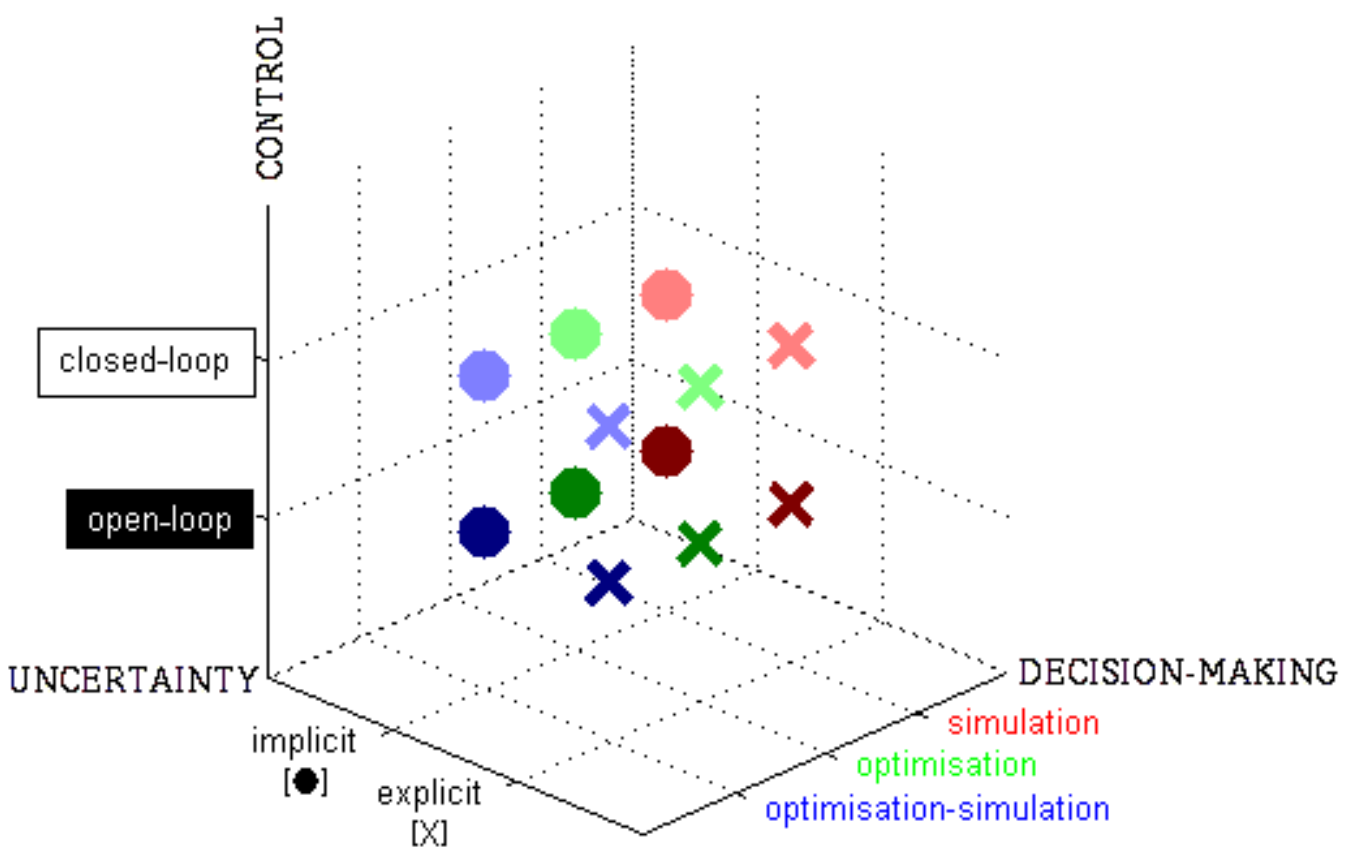

Figure 2.1.: Classification of reservoir decision support tools.

\subsection{Optimisation techniques}

A wide range of optimisation techniques has been applied to determine the optimal operation of power systems since the past century [El-Hawary and Christensen (1979)]. Linear programming (LP) and dynamic programming (DP) are the most popular techniques among them for reservoir systems [Nandalal and Bogardi (2007)]. The former has been normally used for short-term studies [Catalão et al. (2010)] and the latter more frequently for longterm ones [Dias et al. (2010)]. In addition, hybrid optimisation models that combine and take advantage of the best of both techniques have been used with remarkable success [Becker and Yeh (1974); Mariño and Mohammadi (1983); Grygier and Stedinger (1985); Warland et al. (2008); Cristóbal et al. (2009); Abgottspon and Andersson (2012); Philpott et al. (2013)]. What follows is an explanation of the foundations of both techniques as well as a review of their most accurate variants from a hydropeaking point of view.

\subsubsection{Linear programming}

LP [Fourier (1826), cited in Grattan-Guinness (1970)] is a technique to solve optimisation problems in which their objective functions and constraints are linear. According to the type of variables involved in the problem, there are several types of LP [Kall and Mayer (2011)] (Fig. 2.2). These can be all reals (real $L P$ ), all integers (integer $L P$ ) or some reals and some integers (mixed $L P$ ). In addition, the input variables may be treated in two specific manners (as discussed in sec. 1.2): deterministic (deterministic real \{or\} integer \{or\} mixed LP) or stochastic approaches (stochastic real \{or\} integer $\{$ or $\}$ mixed $L P$ ). 


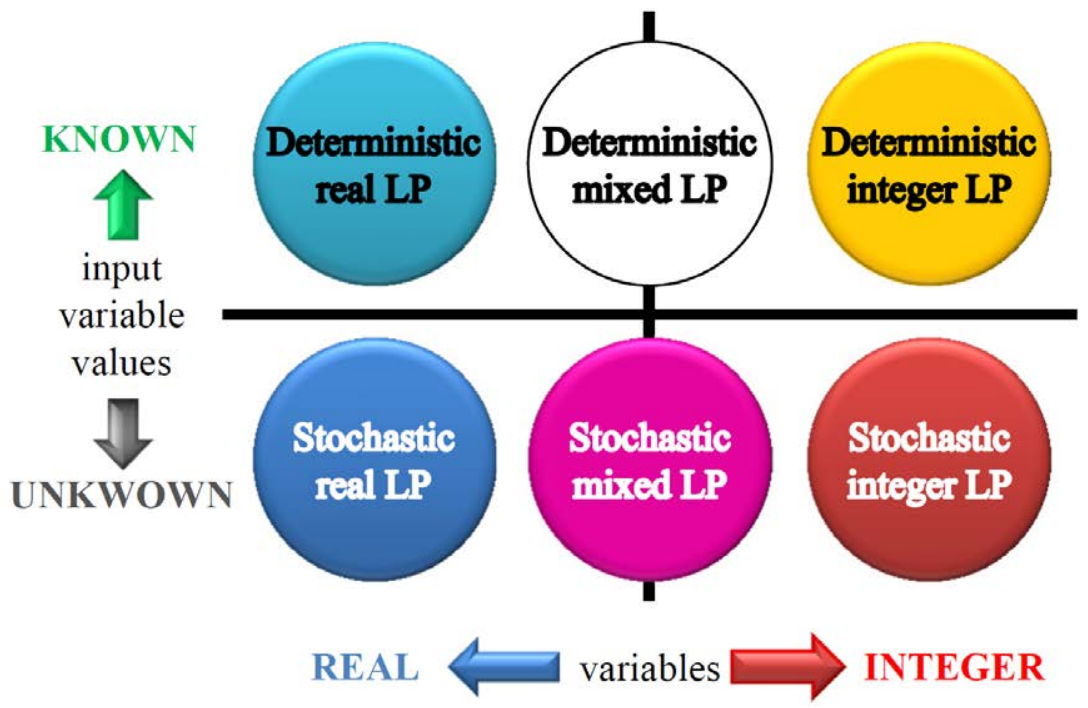

Figure 2.2.: Types of linear programming.

Among its advantages (Tab. 2.1) stand out efficiency to solve big problems and convergence to global optimal solutions [Yeh (1985)]. Furthermore, during the process of resolution, the best solution obtained at each moment is available [Carrión and Arroyo (2006)] being decreasing its speed of approximation to the optimum over time [Carvalho and Pinto (2006)]. Its widespread use is due also to its ease for formulating a broad range of problems with moderate endeavour. This is because a huge number of objective functions and constraints that appear in the practice are linear [Luenberger (2003)]. Unfortunately, the hydro scheduling problem is discrete, non-concave and non-linear (as discussed in sec. 1.2).

The treatment of this last feature represents the main disadvantage of LP (Tab. 2.1) that is the restriction of using linear objective functions and constraints [Rani and Moreira (2010)]. Some techniques to deal with these difficulties are: to add integer variables (in the cases of the real versions) [Dantzig (1954)], to use piecewise linear approximations [Markowitz and Manne (1957)] and to apply successive times this optimisation technique increasing accuracy around the previously obtained solution (successive LP [Griffith and Stewart (1961)]). Unfortunately, the two former techniques increase the computational burden [Babayev and Mardanov (1994)] and the latter may not converge or cause infeasibility [Vargas et al. (1993)]. Prominent examples of these techniques within the hydropower systems field are:

- Yeh et al. (1979): the hydro scheduling of a large system was solved by successive LP.

- Piekutowski et al. (1993): the generation characteristics of a system were modelled by means of several piecewise linear concave curves whose break points were located at null generation, maximum efficiency generation and full gate flow of each plant.

- Conejo et al. (2002): the generation characteristic of a plant was represented through three piecewise linear non-concave curves and five groups of binary variables that allowed to take into account the head effect, non-concavity of the characteristic and the hydro unit on/off status. 
Since all constraints are linear in a LP problem, its feasible region is usually an enclosed region limited by linear hyperplanes [Deb (1995)].

There are several methods to find the global optimum of real linear problems but, from the computational viewpoint, the most competitive are [Illés and Terlaky (2002)]: pivot and interior-point methods.

The idea of pivot method [Dantzig (1948)] is to proceed from one solution to another, both at extreme points of the feasible region, in such a way as to continually improve the value of the objective function until the global optimum is reached. For its part, the interiorpoint method [Karmarkar (1984)] performs the search of this optimum from the inside of the feasible region to its surface. This difference in strategies, in the case of very large problems, provides faster resolution to interior-point method [Illés and Terlaky (2002)].

There are also various methods to solve mixed linear problems. The most relevant are [Bixby (2012)]: branch-and-bound, cutting-plane and branch-and-cut methods.

Branch-and-bound method [Land and Doig (1960)] is based on the following process. Beginning from the global optimum of the real linear relaxation of the mixed linear problem, the feasible region is sequentially divided into smaller and smaller subregions, and both best and worst bounds for the objective function are estimated and updated by any of the abovementioned methods to solve real linear problems. The division continues until all subregions have been explored or until the distance between the best feasible solution and the updated best bound is lower than a specified tolerance.

Cutting-plane method [Gomory (1960)] starts also from the global optimum of the real linear relaxation of the mixed linear problem; likewise calculated by any of the methods for real LP. If it is infeasible within the original problem, then a cut is made, that is, a constraint, which does not exclude any feasible solution, is introduced into the relaxed linear problem. Next, the real linear problem is solved again. This process is repeated until the global optimal feasible solution is found or until the distance between the best feasible solution and the solution of the last relaxed linear problem is lower than a specified tolerance.

Branch-and-cut method [Grötschel et al. (1985)] consists in the combination of the two previous methods. In this combination, after each division and having not been successful in searching a feasible solution within the subregion, a cut is made and it is solved again. If again no solution is found, it branches and the process is repeated until the feasible region is depleted.

Of these three methods the most efficient is the last one [ $\mathrm{Li}$ and Shahidehpour (2005)]. Because of the feasible region is reduced as the process progresses, and only if the problem is very large, the first relaxation can be calculated using the interior-point method and the following can be estimated using the pivot method [Mitchell (2002)].

There are three factors that influence the speed of solving mixed LP problems [Lima and Grossmann (2011)]: problem statement, solver configuration and computer employed.

Regarding the problem statement, a good example of how to accelerate the resolution process is the above-mentioned approach proposed in Piekutowski et al. (1993). In relation 
to the configuration of the solver, and for a given type of problems, trying different methods and carrying out a sensitivity analysis of the optimisation parameters of the solver in the test phases can save much time later [Carvalho and Pinto (2006)]; e.g., in the case of large scale problems, stopping the calculation when the difference between the best integer solution found so far and the best possible integer solution (relative optimality criterion) less than $1 \%$ [Fu and Shahidehpour (2007)]. Finally, not only the processor speed of the computer is important, using of multiple threads in parallel may be very convenient if enough memory is available [Baslis and Bakirtzis (2011)].

\subsubsection{Dynamic programming}

DP [von Neumann and Morgenstern (1944)] is a technique to solve multistage problems based on the optimality principle [Bellman (1954)] which states that a sequence of decisions (policy) is optimal if and only if every decision of it is optimal. Following this principle, a multistage problem can be decomposed, according to its state transition equation, into a series of onestage problems that are solved sequentially over each stage in an iterative process (sense of advance).

Different classifications of DP are possible depending on the chosen characterisation of the features of the multistage problem. The most usual of them are described below: state space, decision making, input variables and sense of advance (Fig. 2.3).

The state space can be considered discrete (discrete DP) or not (continuous DP). This latter choice requires that the system functions are differentiable with respect to state and control [Yakowitz (1982)]. In the case of hydropower operation, it involves not only a substantial simplification of its equations, causing loss of accuracy in the results, but also the use of some techniques embedded in this continuous DP, e.g., mixed LP. Hereinafter only the discrete version will be considered.

The decisions may be taken discreetly (discrete $D P$ ) or continuously (continuous DP) [Bellman and Kalaba (1965)]. What follows only deals with the first version because the stages in multireservoir system operation are uniform time intervals [Labadie (2004)]. As in the literature, the combination of the above-mentioned discrete versions receives also the denomination of discrete DP; this nomenclature is used hereinafter.

The input variables may be considered in two different ways (as discussed in sec.1.2): deterministic (deterministic DP) or stochastic approaches (stochastic DP). In the first case, a single policy is obtained for each state, but in the second one, a decision is obtained for every feasible state and input combination at each stage (steady-state policy) [Loucks and van Beek (2005)].

Under deterministic assumptions, the single policy is directly obtained through one run of DP. However, unless the considered planning period were long enough, under stochastic assumptions and invariant probability distributions between stages for the input variables, it would be necessary to apply several times DP to find the steady-state policy because, at 


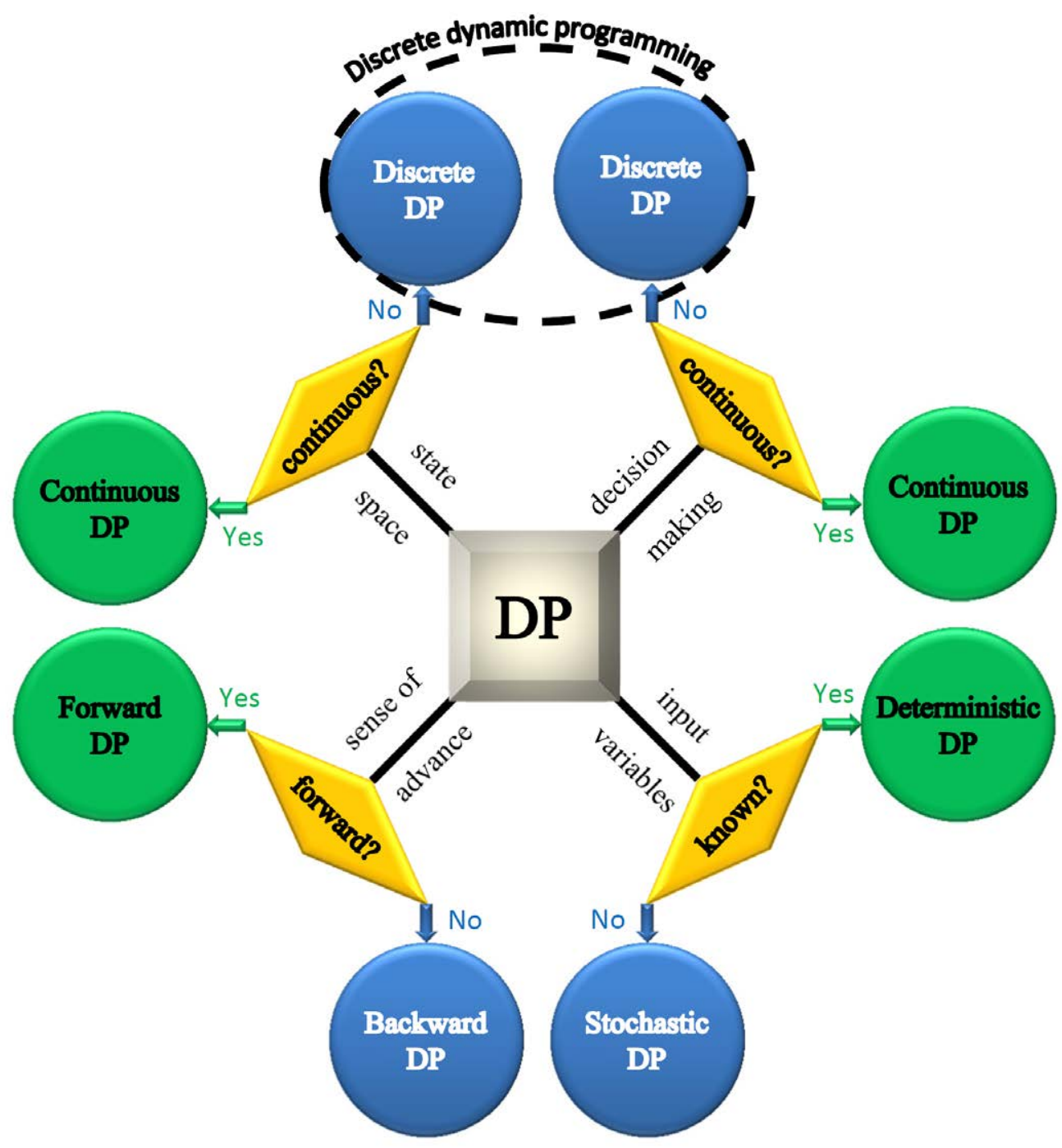

Figure 2.3.: Types of dynamic programming.

each iteration, the results of the previous iteration have to be aggregated at the beginning of the current iteration until convergence is reached [Loucks and van Beek (2005); Nandalal and Bogardi (2007)]. In the case of hydropower plants, the planning period of each iteration should be at least larger than the ratio between the volume capacity of the reservoir and the average annual water inflow (regulation capacity).

There are two criteria of convergence [Nandalal and Bogardi (2007)]: stabilisation of the policy and stabilisation of the expected revenue increment.

The convergence by stabilisation of the policy [Chow et al. (1975)] is achieved when the decision for any state at any stage remains unchanged in each iteration.

The expected revenue increment is, in each iteration, the increase in value of the objective function for any state over the period of study. The stabilisation of the expected revenue increment [Loucks et al. (1981)] is reached when this increase becomes constant and independent of the state. This criterion of convergence is not always achieved in practice [Nandalal and Bogardi (2007)] (as discussed in sec. 2.3.1). 
Regarding the last feature of the problem, the sense of advance can be forwards (forward $D P$ ) or backwards (backward $D P$ ). In a deterministic context, forward advance is more efficient than the backward one when the initial state is fixed and the terminal state is free and and vice versa [Larson (1967)]. However, in a stochastic context, only backward advance has sense since the expectation over the future states has to be considered [Yeh (1985)].

DP is, jointly with LP, the most used technique within the field of multireservoir system operation [Nandalal and Bogardi (2007)]. Among its advantages (Tab. 2.1), it is worth highlighting that its computational burden increases linearly with the number of stages and decreases with the presence of constraints. Moreover, it deals well with the above-mentioned features of hydropower operation [Labadie (2004)] (as discussed in sec. 1.2).

The main disadvantage (Tab.2.1) of this technique is its ingeniously named curse of dimensionality [Bellman (1957)]. It is due to the fact that the number of transitions to be evaluated increases exponentially and potentially as the number of state variables and their discretisations increase respectively.

Several variants of the conventional procedure of discrete DP and some methods, specifically designed to simplify multireservoir problems, have been proposed in order to mollify the said curse.

Concerning the general variants, the most significant are [Labadie (2004)]: coarse grid and interpolation technique, incremental DP and DP successive approximations.

Coarse grid and interpolation technique [Bellman (1957)] reduces the number of transitions that must be evaluated by using big discretisation intervals and then interpolates over this coarse grid. This interpolation can be made by means of different methods, piecewise polynomial cubic splines being one of the most efficient [Johnson et al. (1993)].

Incremental DP [Bernholtz and Graham (1960)] consists in iteratively optimising in the state space neighbourhood (corridor) of one or more given trial policies. At the beginning, a corridor, with an arbitrary width, is built around each trial policy. Then, a new policy is obtained by means of DP and, after that, two possible convergence criteria can be used depending on whether the width of the corridors is kept fixed [Bernholtz and Graham (1960)] or progressively reduced [Turgeon (1982)].

By the first criterion, if the new policy is touching any of the boundaries of the considered corridor, the process is repeated but now around this latter policy and so on until the obtained policy does not touch. By the second criterion, the process stops only when the relative difference between the values associated with consecutive iterations is less than a chosen tolerance. Under this latter criterion, if the chosen tolerance is not reached but the obtained policy does not touch the borders of the considered corridor, the width of the following corridor is reduced and so on until the tolerance is achieved [Nandalal and Bogardi (2007)]. Anyway, this variant has some inherent difficulties such as it is very sensitive to the initial trial policies and the width of the corridor [Labadie (2004)].

DP successive approximations [Bellman and Dreyfus (1962)] decomposes the multidimensional problem into a series of one-dimensional problems by optimising over one state variable 
at a time, with the rest of state variables kept at given current values.

Although the former is the worst of these variants to overcome the curse [Yakowitz (1982); Yeh (1985); Labadie (2004)], the two latter ones share two important limitations. Both are highly dependent on knowledge of the system state with certainty and accordingly they are not adequate for stochastic DP [Labadie (2004)] and their convergence to global optimums is not guaranteed for non-concave and non-convex problems [Yakowitz (1982)] such as hydropower operation ones.

Relating to the methods to simplify multireservoir problems (aggregation-decomposition methods [Ahmed et al. (1965)]), all consists generally in five steps. First, calculating the potential energy of each plant in the system. Second, adding the stored water volume of the system. Third, building a generation function of the whole system that represents its main characteristics. Fourth, optimising the resulting problem. And finally, decomposing the results.

Under deterministic approach, aggregation-decomposition methods are calculated in a single optimisation. However, under stochastic assumptions, the needed number of optimisations depends on how the system has been defined. Thus, these methods can be computed in a single optimisation when the whole system is aggregated in one state variable [Terry et al. (1986)] or in as many as considered reservoirs when the rest of the system is aggregated in one [Turgeon (1980)] or two state variables [Archibald et al. (1997)]. All these approaches severely simplify the system topology [Castelletti et al. (2007)].

State variable discretisation can be realised by many different space filling ways [Crombecq et al. (2011)] but perhaps the most interesting in DP are [Cervellera et al. (2006); Fan et al. (2013)]: uniform, orthogonal array and number theoretic methods.

The discretisation on uniform grid is the most employed distribution and, as its name implies, is homogeneous in the whole state space [Cervellera et al. (2006)]. It provides a good performance when the problem is not too large [Philbrick and Kitanidis (2001)].

Orthogonal array [Kishen (1942)] consists in, from the intervals of a uniform discretisation, selecting randomly states in such a way that, according to an arbitrary number (strength), for any subset of the involved state variables, all possible variable-state combinations appear with the same frequency. In the case that the strength is one, the resulting orthogonal array is called Latin hypercube [Tang (1993)].

Number theoretic methods are a combination of number theory and numerical analysis [Fang et al. (1994)]. This class of methods generates uniformly spaced designs where the domain of interest is normalised to the closed and bounded unit "cube" (with as many dimensions as state variables) for convenience by minimising a discrepancy measure. Star discrepancy is among the most used measures [Fan et al. (2013)] and it can be defined as the maximum deviation between the uniform distribution and the generated by this class of methods [Niederreiter (1992)]. The generation of the states is based on low discrepancy sequences such as, e.g., Hammersley [Hammersley (1960)], Halton [Halton (1960)] and Sobol [Sobol (1967), cited in Atanassov (2003)] sequences. Both this way and the previous one 
reduce the computational burden of high dimensional problems [Cervellera et al. (2006)].

Regardless of the way chosen, the discretisation of $\mathrm{V}$, which is probably the most important state variable in reservoir operation [Goulter and Tai (1985)], has two important features. On the one hand, a coarse discretisation produces high skewness in the storage probability distribution functions and influences the optimal policy [Klemeš (1977); Goulter and Tai (1985)]. On the other hand, the decreasing size of its intervals does not automatically guarantee better results and the improvement, if any, does not occur continuously [Bogardi et al. (1988)].

A specific criterion for selecting the discretisation of this variable is to set its interval shorter than the volume equivalent to the smallest water inflow [Ratnayake and Harboe (2007)]. In this context, several studies have shown numerical results that suggest as a good discretisation that in which the number of V states per reservoir is between 9 and 33 [Moran (1959); Doran (1975); Goulter and Tai (1985); Bogardi et al. (1988)].

Finally, since V in a system are related to each other [Durán et al. (1985)], some combinations of these are really unreasonable, e.g., one reservoir will not be empty while the others are full. Thereupon it may be convenient to reduce the state space around a corridor of reasonable volumes [Stedinger et al. (2013)].

Table 2.1.: Advantages and disadvantages of linear and dynamic programmings in hydropower operation.

\begin{tabular}{|c|c|c|}
\hline TEChNIQUeS & ADVANTAGES & DISADVANTAGES \\
\hline LP & $\begin{array}{l}\text { - Convergence to global optimal solutions. } \\
\text { - Ease of formulating. } \\
\text {. Efficiency to solve big problems. }\end{array}$ & $\begin{array}{l}\text { Restriction of using linear } \\
\text { objective functions and } \\
\text { constraints. }\end{array}$ \\
\hline $\mathrm{DP}$ & $\begin{array}{l}\text { Computational burden increases linearly } \\
\text { with stages and decreases with } \\
\text { constraints. } \\
\text { · Discreteness, non-concavity and } \\
\text { non-linearity immunity. }\end{array}$ & $\begin{array}{l}\text { Computational burden } \\
\text { increases exponentially } \\
\text { with state variables and } \\
\text { potentially with state } \\
\text { space discretisation. }\end{array}$ \\
\hline
\end{tabular}

\subsection{Input variables}

Any dynamics in nature is stochastic to some degree [Cvitanovic et al. (1999)] and hydropower operation is not an exception [Little (1955)]. Markov chains are the first, most important and simplest mathematical models for random phenomena evolving in time [Norris (1998)] and they have been used extensively in reservoir operation models [Yakowitz (1982); Yeh (1985); Labadie (2004); Loucks and van Beek (2005); Nandalal and Bogardi (2007)]. There are many input variables that affect hydro scheduling [Forsund (2007)] but probably the most significant are: water inflow [Belsnes and Fosso (2008)], energy price [Fosso et al. (1999)] and in some basins [Torcellini et al. (2004)], especially over the long-term [Yu et al. (1998)], evaporation [Teixeira and Mariño (2002)]. What follows deals with these input variables but before an explanation of Markov chain theory is given. 


\subsubsection{Markov chain theory}

Markov chains are frequently applied in optimisation of multireservoir systems and considered convenient for this function [Loucks and van Beek (2005)] despite showing low persistence and not capturing the variance of some of their input variables [Stedinger et al. (2013)]. A Markov chain [Markov (1906), cited in Basharin et al. (2004)] consists in a set of states (classes) and a set of branded transitions between these classes. After a halt in a class, the Markov chain will make a transition to another class. Such transitions are branded with either probabilities of transition (discrete-time Markov chain) or rates of transition (continuous-time Markov chain) [Bolch et al. (2006)]. What follows only deals with the discrete type because the stages in multireservoir system operation are uniform time units [Labadie (2004)].

In order to model a random variable during a period of time in which it has a repetitive pattern (time cycle) by means of a Markov chain, it is necessary to make two assumptions [Kemeny and Snell (1960)]: each transition probability of the variable depends only on its recent past (markovianity) and remains constant over the time cycle (stationarity).

Markovianity is the critical feature of a Markov model [Meyn and Tweedie (1993)] and may manifest itself with different orders of dependence. The order (lag) of a Markov chain is the number of time units from the current time whose variable values are involved in the definition of the actual variable value [Lowry and Guthrie (1968)]. With the aim to operate with ease, the transition probabilities of a random variable are usually collected in an array (transition probability array) of order equals to one plus its lag [Preda and Balcau (2008)].

Stationarity is the necessary condition to guarantee that the policy of a multistage problem will become stable after a certain number of cycles [Nandalal and Bogardi (2007)]. This property is recognised within a Markov chain when its transition probability arrays do not change over the cycle [Wallace and Bassuk (1991)].

There are several types of Markov chains [Kolmogoroff (1936), cited in Mazliak (2007)] but there is only one that ensures that the stable policy of a multistage problem, that has been derived from it, will be the global optimum [Howard (1960)]: ergodic Markov chain. This type has the three following properties [Solow and Smith (2006)]: each of its classes can eventually be reached from every other class (irreducible), the expected return time to each class is finite (positive recurrent) and starting in each class, there exists no regular period at which the class cannot be reached (aperiodic).

The violation of the ergodicity can be caused by a large number of zero elements in the transition probability arrays, and additionally can provoke the failure in the stabilisation of the expected revenue increment (as discussed in sec. 2.2.2). Those zero elements are inaccuracies because of lack of data [Nandalal and Bogardi (2007)].

This problem can be circumvented by two compatible methods [Nandalal and Bogardi (2007)]: smoothing and, which may be called, discretisation thickening.

The smoothing method [Bellman and Dreyfus (1962)] consist in replacing the zero elements by small quantities which are subtracted from the non-zero elements. This replacement is 
done only when more than half the elements of a subarray of a discretisation class are zero and must be done such that the sum of the transition probabilities of this subarray equals one [Nandalal and Bogardi (2007)].

The discretisation thickening method [Nandalal and Bogardi (2007)] is based on the idea that the number of zero elements depends on the ratio between the number of discretisation classes of the considered variable and its total number of available historical observation data. It is convenient that the total number of data be greater than half the cubic of its classes.

Regarding the determination of the lag of a Markov chain, the most common tests are [Wilks (2006)]: Akaike [Akaike (1971)] and Bayesian [Schwarz (1978)] information criteria. Both criteria are based on the log likelihood functions for the transition probabilities of the Markov chains. These log likelihoods depend on the transition counts and the estimated transition probabilities [Wilks (2006)]. The Akaike criterion tends to be less conservative, generally picking higher orders than the Bayesian one when the results of the two tests differ. The use of this last criterion can be preferable for sufficiently long time series (between 100 and 1000 data) depending on the nature of the serial correlation [Katz (1981)].

Finally, regarding the variable discretisation, in addition to the considerations mentioned in the previous section, it is advisable to maintain its extreme values, both low and high, to improve the performance of the system operation [Kim and Palmer (1997); Mo et al. (2001)].

\subsubsection{Water inflow}

Water inflow can be defined as the volume of water that arrives in a natural way (by rainfall, runoff and snowmelt) at a reservoir per stage. It is the most important input variable in hydropower operation [Belsnes and Fosso (2008)], especially when the storage levels are nearly empty or nearly full [Fleten et al. (2009)], and its importance lies in its influence on V and the net head [Little (1955)].

The use of Markov chains to model monthly water inflow [Stedinger et al. (1984)], as well as daily [Jimoh and Webster (1996)] and weekly [Deni and Jemain (2012)] rainfall, is widespread in the literature. Generally, in a multireservoir system, water inflows are spatially and temporally correlated and only for very long time steps, of perhaps one year or more, these correlations do not occur [Philbrick and Kitanidis (1999)]. With the aim of reducing the number of water inflow variables to be modelled in a this kind of systems, it has been applied principal component analysis providing good results [Saad and Turgeon (1988); Archibald et al. (2006)]. This is possibly the most broadly used multivariate statistical technique in the atmospheric sciences [Wilks (2006)].

Principal component analysis [Pearson (1901)] consists in transforming an original set of interrelated variables into another smaller one formed by new uncorrelated variables which are linear combinations of the initial ones. This transformation has to be made with the goal of keeping as much as possible of the variability present in the original set. 


\subsubsection{Energy price}

Energy price, in this context, represents the price received by producers for selling energy. In the case of deregulated electricity markets, this price is generated from one day to another and would represent the marginal value of produced energy at each hour in the market [Fosso et al. (1999)]. Its behaviour is characterised by: non-constant mean and variance, multiple seasonality, calendar effect, and high frequency, volatility and percentage of unusual values [Nogales et al. (2002)]. Despite the difficulty of modelling these features, given the importance to price-takers [Gjelsvik et al. (2010)], there is a large variety of techniques that try to do it [Bunn (2004)].

In mid- and long-term hydro scheduling of a system composed only of price-takers, one of these techniques consists in using the average of energy price during the considered stage by means of a Markov chain [Gjelsvik et al. (1999)]. This technique, on the one hand, is probably the simplest and computationally less burdensome one, but, on the other hand, usually underestimates revenues because it disregards hydropeaking [Olivares and Lund (2011)]. An alternative to model this input variable is based on the consideration of different scenarios of hourly values [Abgottspon and Andersson (2012)]. Of course, it is also possible to use both [Helseth et al. (2013)]. Finally, if, after a statistical analysis, there is a significant correlation between this input variable and others (usually water inflow), this correlation must be considered [Löhndorf et al. (2013)].

\subsubsection{Evaporation}

Evaporation is the gradual solution of water in air [Hamilton (1767)]. This process is influenced by many factors, such as solar radiation, vapour pressure deficit, relative humidity, wind speed and air temperature [Xu and Singh (1998)]. Depending on the climate where its magnitude need to be determined, it can be estimated by many different equations which, in turn, may require very diverse data. This circumstance usually makes it difficult to decide which is the most proper one for a given location [Winter et al. (1995)].

Among the principal factors that inevitably conditions the choice of a particular equation is the available data [Warnaka and Pochop (1988)]. As air temperature is the easiest and cheapest data to measure among the above-mentioned [Ortuño et al. (2010)], the equations that require only this input (temperature-based equations) are frequently used [Zhang et al. (2012)]; sometimes even offering better results than more complex equations [Rosenberry et al. (2007)]. The main drawback of the temperature-based equations is that its accuracy depends on the correlation between air temperature and solar radiation of the place where they were developed [Juday et al. (2011)]. Therefore, if this correlation varies from a place to another, then their parameters must be recalibrated [Xu and Singh (2001)].

Regardless of the equation used to calculate the evaporation rate, reservoir evaporation loss is commonly estimated as the product of this rate and the average water surface during the considered period [Loucks et al. (1981)]. Therefore this loss is related to V. 


\subsection{Generation characteristics}

The generation characteristic (also known as water rate curves, characteristic curves and production function [Pérez-Díaz et al. (2010)]), of both a single hydro unit and a whole peak hydropower plant, describes the relationship between its released flow, its available head, and its generated power [Conejo et al. (2002)]. Its graphical representation (power characteristic surface) is, therefore, three-dimensional and it can be modelled by a set of usually power-discharge curves (performance curves), each for a specified head [García-González and Castro (2001)]. It is the most crucial modelling aspect in daily hydro scheduling [Nilsson and Sjelvgren (1997b)].

In the case of a single hydro unit, its power characteristic surface is usually provided by its manufacturer and so the hydraulic losses in the plant conduits are not included [Maruzewski et al. (2012)]. Additional information that should be included in this case, if any, are the undesirable generation levels (forbidden zones) of the hydro unit [Finardi and da Silva (2005)].

In the case of a whole plant with several hydro units, its generation characteristic can be modelled individually per hydro unit [Chang et al. (2001)] or as the result of the allocation of water among the hydro units that maximises total plant power output for given flow discharged and gross head (instantaneous load dispatch) [Allen and Bridgeman (1986)]. A key aspect of this allocation is that the hydro unit start-up and shut-down costs [Nilsson and Sjelvgren (1997a)] must be contemplated [Siu et al. (2001)].

Given its importance in short-term and that LP (as discussed in sec. 2.2.1) is the most used technique in this planning period, an explanation of the main types of models of the performance curves used within LP based hydro scheduling models is included in what follows. After, a description of the cases of hydropower instantaneous load dispatch is provided.

\subsubsection{Performance curves}

There are several models to represent performance curves in LP based hydro scheduling models. The most representative can be enumerated, in order of increasing accuracy [Borghetti et al. (2008); D'Ambrosio et al. (2010)], as follows:

1. A piecewise linear concave function with breakpoints at the points corresponding to minimum power, local best efficiencies and maximum total flow [Habibollahzadeh and Bubenko (1986)].

2. Several piecewise linear non-concave functions [Conejo et al. (2002)].

3. A dotted non-concave mesh (one-dimensional method) [D'Ambrosio et al. (2010)].

4. A triangular non-concave mesh (rectangle method) [García-González and Castro (2001)].

The choice among these models depends on the trade-off between the quality of the representation and the computational time on its evaluation [D'Ambrosio et al. (2010)]. On the one hand, models with one linear function neglect the variation of the head [Conejo et al. 
(2002)] which is only recommended for relatively large reservoirs and short-term planning periods. On the other hand, numerical results have shown that one-dimensional method is considerably better than several other functions [Borghetti et al. (2008)] but, in comparison with rectangle method, it improves very little the solution at the expense of spending a lot of time [D'Ambrosio et al. (2010)].

\subsubsection{Instantaneous load dispatch}

Depending on the number of hydro units, whether or not the turbine models are equal, they are fed by the same penstock or have the same hydraulic losses, and their individual generation characteristics are concave, three different cases can be distinguished to determine the instantaneous load dispatch of a peak hydropower plant (Tab. 2.2) whose descriptions are as follows:

Table 2.2.: Instantaneous load dispatch cases of a peak hydropower plant.

\begin{tabular}{|c|c|c|c|c|}
\hline $\begin{array}{l}\text { HYDRO } \\
\text { UNITS }\end{array}$ & $\begin{array}{l}\text { TURBINE } \\
\text { MODELS }\end{array}$ & $\begin{array}{c}\text { HYDRAULIC } \\
\text { LOSSES }\end{array}$ & $\begin{array}{c}\text { POWER CHARACTERISTIC } \\
\text { SURFACES }\end{array}$ & Cases \\
\hline 1 & $\forall$ & $\forall$ & $\forall$ & $\mathrm{A}$ \\
\hline \multirow{4}{*}{$>1$} & \multirow{3}{*}{$=$} & \multirow{2}{*}{$=$} & concave & B \\
\hline & & & non-concave & \multirow{3}{*}{$\mathrm{C}$} \\
\hline & & $\neq$ & $\forall$ & \\
\hline & $\neq$ & $\forall$ & $\forall$ & \\
\hline
\end{tabular}

- Case A refers to a hydro plant with a single unit. The instantaneous load dispatch is obviously straightforward and the resulting generation characteristic consists simply in adding the hydraulic losses of the plant to the hydro unit power characteristic surface.

- Case B refers to a hydro plant with several identical hydro units with a concave power characteristic surface and equal hydraulic losses. From the Karush-Kuhn-Tucker conditions [Karush (1939)], it is possible to demonstrate that the solution is such that all units in operation must have the same partial derivative of the flow with respect to the power. Since for each flow and gross head, there may exist several feasible combinations of units in operation, it is necessary to evaluate and compare to each other the maximum power of each feasible combination in order to determine the best solution.

- Case $\mathrm{C}$ encompasses the rest of the situations. Many optimisation techniques have been applied to solve this case being DP (as discussed in sec. 2.2.2), due to its effectiveness [Yi et al. (2003)], one of the most common among them [Padhy (2004)]. 


\subsection{Hydro scheduling models with minimum flows and maximum ramping rates}

What follows is a revision of the most hydro scheduling models that have considered $\varphi$ and $\rho$ jointly according to their optimisation techniques.

\subsubsection{Linear programming models}

LP (as discussed in sec. 2.2.1) has been the most applied technique in hydro scheduling models with $\varphi$ and $\rho$. It may be highlighted the following:

- [Shafer and Labadie (1978); Colorado State University (2010)]

MODSIM $^{\mathrm{TM}}$ is a river basin decision support tool, elaborated at the Colorado State University (USA), for developing regional strategies for different purposes, such as: short-term water management, long-term operational planning, drought contingency planning, etc. It has been applied to several existing power systems, especially at the United States, and it takes advantage of the network nature of the basin (network flow programming [Kirchhoff (1845), cited in Ahuja et al. (1993)]), to obtain its output.

MODSIM $^{\mathrm{TM}}$ considers the influence of the head on the power generation, distinguishes between on- and off-peak periods and is able to model both water inflows and consumptive and instream flow demands as stochastic variables. The time step can be monthly, weekly or daily and the planning period can reach many years.

- [Veselka et al. (1995)]

This technical report, developed at the University of Chicago (USA), was redacted to study, among other topics, hydropower operational constraints in combination with alternative contractual commitments at the Salt Lake City Area Integrated Projects (USA). A couple of models, one based on simulation and one based on optimisation, were devoted to this specific purpose.

The mission of the simulation model was to project future monthly hydropower plant capabilities in probabilistic terms. It had a planning period of 150 years and monthly time steps. Combinations of 85 historical series of water inflows, 4 contractual commitments and 3 operational scenarios were simulated considering evaporation and snow pack. Because of the length of the time step and with the aim of introducing the effect of the hourly constraints on the model, a geometric algorithm was applied in three real annual series of water inflows and interpolated in the rest of them later. This algorithm, that distinguished between off-peak and on-peak periods, worked under the assumption that on-peak periods lasted four hours.

The optimisation model, based on real LP, was used to minimise the system's net operating costs. From the total monthly energy generation of each plant estimated by the simulation model and the energy prices determined by another model, this optimisation model was employed with hourly time step for one week per month in combinations of three annual 
series, four contractual commitments and three operational scenarios. Monthly results were estimated by scaling up aggregated weekly results.

Among the results showed in this report, it is worthy to mention that the operational scenarios affected monthly but not annual water release volumes.

- [Mo et al. (1997); Belsnes et al. (2013)]

$\mathrm{SHOP}^{\mathrm{TM}}$ is a program for short-term hydro operation planning developed by SINTEF Energi AS and currently used at many existing systems. This tool, based on successive real or mixed LP, is deterministic and considers water inflows and energy prices. The time step and the planning period are flexible and the endpoint reservoir level can be described by water values or specified endpoint volumes. The hydro units can be defined by any number of performance curves, the start-up costs and the head losses in water tunnels being also modelled.

- [Chang et al. (2001)]

This paper described a model, based on mixed LP, for the short-term hydro scheduling of multireservoir systems in deregulated markets. The model was tested in the hydro resources of two systems: Southern Generation Group of the Electricity Corporation of New Zealand Limited and Swiss Rail.

Half hourly step and daily planning period were used in the first system and hourly step and planning periods ranging from one day to one week in the second one. The operating states of the hydro units were represented by binary variables and a generation characteristic for each unit based on one piecewise linear concave function with breakpoints at the points corresponding to minimum power, local best efficiencies and maximum total flow were implemented in the model. Both water inflows and energy prices were estimated by forecasts.

- [Eschenbach et al. (2001); Zagona et al. (2001); University of Colorado (2014)]

RiverWare $^{\mathrm{TM}}$ is a reservoir management modelling framework for optimisation and simulation of multipurpose reservoirs developed at the University of Colorado (USA). Nowadays, it is applied to several existing power systems such as the Tennessee Valley reservoir system (USA). The operation of this particular system is the result of the combined action of two optimisation models which are updated every day considering both water inflow and energy price forecasts.

The first model defines the input variables of the second one which are the daily F. It has a six hourly time step, covers a two-weekly planning period and, besides the updated forecasts, needs as inputs the water values at the time horizon. The generation characteristics are modelled through a single piecewise linear concave function for each plant.

The second model, in turn, calculates the hourly power generation from each hydropower plant. It uses an hourly time step and covers a planning period of two days. This model can represent hydro unit on/off status and forbidden zones as well as the generation characteristic of each hydropower plant by means of the rectangle method, but at the expense of a high computational cost. 
After each optimisation, based on real or mixed LP, a simulation is run to determine how much error was introduced into the optimisation model due to approximations.

In order to reduce the computation time, RiverWare ${ }^{\mathrm{TM}}$ uses a heuristic procedure that gradually imposes integer restrictions starting from the first time steps forward. After each iteration, the optimal integer values for the time steps, in which integer restrictions were imposed, are locked in. The process continues until all time steps are solved with integer restrictions, holding fixed the time horizon.

- [Rauscher et al. (2002); Robitaille et al. (2005); Hatch Ltd. (2013)]

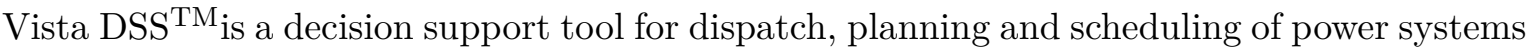
developed by Hatch Ltd. and used at many existing systems at the present time. This tool is composed, among other parts, of two models that are based on real and mixed LP and, respectively, focused on the long and short-term scheduling.

The long-term model estimates the water values at the end of each stage of the shortterm one. Its planning period ranges between one or more years and its time step increases progressively along the planning period from one week to one month. It considers between five and nine scenarios of water inflows and one scenario of costs or prices. The time step can be decomposed up to twenty off-peak and on-peak periods. This model is usually run at least once every week with updated information about the system.

The short-term model maximises the revenue or minimises the system generation costs using the previous water values over a planning period of one or two weeks. By means of successive mixed LP, this model takes into account, usually on an hourly basis, the influence of the head on the power generation as well as the operating states and forbidden zones of the hydro units. With the aim of ensuring the feasibility of each problem considered, slack variables for constraints are included in the problem formulation.

- [Kotchen et al. (2006)]

This study, based on real LP, was proposed to conduct a benefit-cost analysis of a relicensing agreement for two peak hydropower plants in the Manistee River (USA). This agreement implied a change in the operation, from hydropeaking to operate according to the natural flow present at any time (run-of-river). The reduction in the annual benefit of the plants was calculated as the annual cost of the necessary replacement power supplied by thermal power plants. It used an annual time step covering a planning period of twelve years. A couple of different energy marginal costs, peak and off-peak costs, were used and the characteristic generation considered only one head value.

- [Borghetti et al. (2008)]

This paper compared several mixed LP models for the short-term hydro scheduling of a single pump-storage hydropower plant in a deregulated market. The distinguishing feature of the models was the way to represent performance curves which were basically based on several 
piecewise linear non-concave functions or on the rectangle method (the main conclusions of this comparison have been summarised in sec.2.4.1). Regarding of other features of the models, three different scenarios were studied in a deterministic manner, the operating states of the hydro units were contemplated and the planning period used was one week divided into hourly steps.

- $[$ Olivares (2008)]

This thesis developed two revenue-driven optimisation models to operate under environmental constraints. The first model, based on real LP, neglected the influence of the head on the power generation as well as the hydro unit on/off status, whereas the second one did not. Both used hourly time steps but the first covered one day and the second one week. Both considered, in a deterministic way, the water inflows; the first one treated also, in the same way, the energy prices, while the other considered the frequency curve of these energy prices.

The dissertation states that $\varphi$ forces a plant to generate electricity during less valuable hours and $\rho$ causes more uniform hourly F. Among its results, it is worthy to mention that the daily revenue losses of the studied plant showed an almost linear and quadratic growth as $\varphi$ and $\rho$ became more restrictive, respectively.

- [Pérez-Díaz and Wilhelmi (2010)]

This paper proposed a model, based on mixed LP, to assess the economic impact of environmental constraints in the short-term operation of a peak hydropower plant. It was a daily revenue optimisation model with hourly time step. The generation characteristic was modelled by means of several piecewise linear non-concave functions and both the energy prices and the water inflows were considered deterministic. Its application to a real plant located in the Northwest of Spain resulted in a similar behaviour of the daily revenue losses to that detected in Olivares (2008).

- [Alemu et al. (2011)]

Storm ${ }^{\mathrm{TM}}$ was a decision support tool that was developed for a small system with two reservoirs and one peak hydropower plant, associated to one of the reservoirs, located in the Sultan River (USA). It was composed of two models: one based on simulation and the other based on optimisation.

The simulation model, with daily time step, was created to provide guidance for long-term system operation and was based upon historic reservoir operating policies. The optimisation model, based on real LP, was designed, among several objectives, to maximise the value of the energy produced over a shorter planning period and used forecasts of water inflows and energy prices.

- [Pérez-Díaz et al. (2012)]

This paper studied the contribution of a re-regulation reservoir to fulfilling $\varphi$ and $\rho$ while reducing the economic impact of said constraints. For that purpose, four revenue-driven 
models, based on mixed LP, were applied in a real hydropower plant located in the Northwest area of Spain. The first one calculated the optimal operation of a hydropower plant without considering any environmental constraint; the second one calculated this operation while fulfilling $\varphi$ and $\rho$; the third one considered the existence of a re-regulation reservoir right downstream of the main reservoir; and the last one considered the addition of pumping capability between both reservoirs. All the models used an hourly time step covering a planning period of one month and the generation characteristic was modelled by one piecewise linear concave function because of the small range of variation of the operating heads in comparison to the total gross head of the considered hydropower plant.

A sensitivity analysis of the economic impact of $\varphi$ and $\rho$ were carried out in a deterministic way for three water inflow scenarios (dry, normal and wet) and one real energy price scenario. The results showed a similar evolution of those obtained in Olivares (2008) and Pérez-Díaz and Wilhelmi (2010).

- [O'Neil et al. (2013); Steinschneider et al. (2014)]

These studies used a real linear optimisation model to examine trade-offs between the maintenance of natural flows and common operational objectives of the main dams at the Connecticut River basin (USA). The model minimised the value of penalties due to the deviations from the natural flows minus the income of the hydropower plants. Its time step and planning period were daily and yearly respectively. Energy prices were aggregated and averaged for each day of the year and many water inflow projections were considered in order to evaluate the aforementioned trade-offs facing the climate change.

- [Rheinheimer et al. (2013)]

This study developed a model, based on real LP, to estimate the generation and revenue variations caused by environmental constraints and climate warming in five plants in the Yuba River (USA). It was a two-yearly model with weekly time step in which the net head and generation efficiency were assumed constant for each plant. The revenue losses obtained in this research showed the same behaviour as in Olivares (2008), Pérez-Díaz and Wilhelmi (2010) and Pérez-Díaz et al. (2012).

- [Diniz and Souza (2014)]

This paper suggested a deterministic real LP model to minimise the costs of systems with hydro and thermal plants and it was tested with the Brazilian system composed of 141 hydro plants and 96 thermal plants. The model was focused on river level and routing constraints and the coordination between short- and mid-term planning. In order to improve this coordination, the objective function took into account the water value provided by the moving water between reservoirs as if these volumes had already reached their respectively downstream dams. With two planning periods of one day and one week, both discretised in hourly time steps, the model also considered the generation characteristic of each hydro plant by means of a piecewise linear function in which the tailrace elevations were included. 
A novel contribution of this paper was the way in which $\rho$ was modeled. This way, based in the river level variations (usual practice in USA law), was carried out approximating the function that relates $\mathrm{F}$ to the level variations (rating curve) through a linear regression.

- [Olivares et al. (2015)]

In this study, a hydrothermal model, based on mixed LP, was used to assess the economic and environmental efficiency of $\varphi$ and $\rho$ on the Central Interconnected System in Chile. The model minimised the sum of the operational costs of the thermal and renewable plants, the cost of unserved load at the buses of the grid and the water values of the hydropower plants (these water values were given without considering $\varphi$ and $\rho$ ). Its planning period and time step were weekly and hourly, respectively. The net head of each plant was updated at the beginning of each week and its generation characteristic was modelled by one linear segment.

A sensitivity analysis of $\varphi$ and $\rho$ were carried out in a deterministic way for three water inflow scenarios (dry, normal and wet) and one energy price scenario. The authors stated that while $\rho$ did not cause significant economic impact because the global flexibility of the system, $\varphi$ was more costly by reducing $\mathrm{V}$ in the reservoirs.

\subsubsection{Non-linear programming models}

Non-linear programming [Kuhn and Tucker (1950)] is a technique to solve optimisation problems in which the objective functions and/or constraints are non-linear [Bertsekas (1999)]. Although this technique is very convenient for modelling problems, its convergence to global optimal solutions is usually not guaranteed [Martin (1983)]. Some of the non-linear programming based hydro scheduling models that have considered $\varphi$ and $\rho$ are the following:

- [Harpman (1999)]

This monthly peak shaving model with hourly time step was developed to estimate the annual economic cost of environmental constraints at Glen Canyon dam in the Colorado River (USA). A non-linear function to consider the influence of the head on power generation and a representative annual water inflow scenario were used to calculate the optimal operation. Thereafter, a simulation, based on the mean daily on- and off-peak energy prices, was carried out to obtain the results. These indicated that restrictions on maximum and $\varphi, \rho$ and daily flow changes may produce a significant fall in the annual economic value of hydropower.

- $[$ Niu and Insley (2010)]

This model was created to study the economic impact of $\rho$ in the short-term. With the aim of maximising the revenue, its features were: hourly time step, five-daily planning period, influence of the head and energy prices as in Harpman (1999), and a constant hourly water inflow. It was applied on a medium sized plant in Ontario (Canada) and, as in Olivares (2008), Pérez-Díaz and Wilhelmi (2010), Pérez-Díaz et al. (2012) and Rheinheimer et al. (2013), the revenue losses of this plant showed a quadratic growth as $\rho$ became more restrictive. 
- [Babel et al. (2012)]

This study analysed the impact of five alternative scenarios of operation on the energy production of two plants in cascade in the La Nga River basin (Vietnam) by means of a simulation model. In one of these scenarios, the decision rules of the upstream plant were previously obtained by optimisation considering the downstream one as a run-of-river plant. The used optimisation model, with two-yearly planning period and monthly time step, contemplated the influences of the head as well as the hydraulic losses on the power generation and also the effect of evaporation on $\mathrm{V}$.

- [Pousinho et al. (2012)]

This paper suggested a model, based on mixed quadratic programming, for the short-term scheduling of a Portuguese hydro system with seven cascaded plants. It was a daily revenue optimisation model with hourly time step. The generation characteristics were represented by non-linear functions but, although the on/off behaviour of the plants were modelled, the hydro unit on/off status were not considered. The water inflows were deemed deterministic and the energy prices were shaped by twenty scenarios. Different levels of risk assumed by the producers were compared.

\subsubsection{Dynamic programming models}

In what follows, some of the models based on DP (as discussed in sec. 2.2.2) that have been considered both $\varphi$ and $\rho$ are described:

- [Edwards et al. (1999)]

This paper applied a deterministic model and subsequently simulations to examine, under different environmental constraints scenarios, the monthly hydro scheduling of Flaming Gorge plant in the Green River (USA). The optimisation model, based on continuous DP, used half-daily time steps, a linear function of $\mathrm{V}$ to represent the influence of the head on power generation, and the same two energy prices each day distinguishing between on- and off-peak periods. The simulations, in turn, were carried out with the same data but hourly time step. The paper showed that $\rho$ can increase the release volume during off-peak periods.

- [Petreski (1999)]

Allocate $^{\mathrm{TM}}$ and Wopro ${ }^{\mathrm{TM}}$ were proposed as hydropower decision support tools based on optimisation for real-time multireservoir operation and planning. The purpose of Allocate ${ }^{\mathrm{TM}}$ was the determination of the instantaneous load dispatch and the objective of Wopro ${ }^{\text {TM }}$ could be selected among different options, e.g., maximisation of energy, maximisation of benefits or minimisation of costs in systems with hydro and thermal plants. Discrete DP was applied in two steps: the first, with a coarse grid, to produce a near optimal solution and the second, around the previous operating policy, to refine the solution. 
Wopro $^{\mathrm{TM}}$ admitted time steps from 1 minute to 1 month supporting up to 600 time steps. It was able to solve problems for up to three reservoirs in series or parallel connection. Water inflow forecasts could be generated within the program or imported from an external source. Hydraulic losses could be defined for common or separate conduit systems and the net head was calculated in each time step.

- [Chen and Forsyth (2008)]

The objective of this weekly hydro scheduling model with hourly time step, based on continuous DP, was to maximise the revenue in the short-term. The influence of the head on power generation was represented by a non-linear function, the water inflow was considered constant and the nature of the energy prices was considered through an one-factor model. As in Olivares (2008), Niu and Insley (2010), Pérez-Díaz and Wilhelmi (2010), Pérez-Díaz et al. (2012) and Rheinheimer et al. (2013), numerical results obtained in the application of this model from a single hydropower plant showed a quadratic growth of the revenue losses as $\rho$ became more restrictive.

- [Niu and Insley (2013)]

With the same objective, most assumptions and case study as in Niu and Insley (2010) but by means of an approach similar to which was used in Chen and Forsyth (2008), this model applied a fully implicit finite-difference scheme to solve the weekly scheduling problem in which the energy prices were modelled by a regime-switching model. In this work, a constant cost of hydropower generation was assumed for the hydro station. The results showed that the effect of $\rho$ on revenue mostly depends on the level of the price relative to the cost of generation.

\subsubsection{Other models}

Although rarely with $\varphi$ and $\rho$, combinations of any of the aforementioned techniques or others have also been used in hydro scheduling models, such as: genetic algorithms [Box (1957)] and progressive optimisation algorithm [Howson and Sancho (1975)].

Genetic algorithms combine the principle of natural selection [Wells (1818)], based on the survival of the fittest, with genetic processes copied from nature [Holland (1975)], such as crossover, inheritance, mutation and selection. On the one hand this technique is suitable for non-concave and non-linear problems such us hydropower operation ones, but on the other hand, in addition to its large computational burden, its convergence to global optimal solutions is not guaranteed [Labadie (2004)].

Progressive optimisation algorithm is a technique, based on an a priori deduction from the optimality principle (as discussed in sec.2.2.2), to solve multistage problems by means of decomposing them into series of overlapping two-stage problems that are solved successively [Howson and Sancho (1975)]. Among its advantages are non-linearity inmunity and low 
computational burden, but its main drawback is that the convergence to global optimum is not ensured [Cheng et al. (2012)].

In what follows, some of these models are described:

- [Liu et al. (2011)]

The objective of this study was to derive optimal reservoir refill rules for a multipurpose reservoir considering flood control and comprehensive utilisation benefits. With this aim, an iterative method composed of three models was proposed and applied to the Three Gorges reservoir in the Yangtze River (China). These models were developed for simulation, optimisation and testing of the feasibility of the obtained solutions respectively. Both the simulation and optimisation models considered four objective functions: minimise flood risk, maximise hydropower generation, maximise refill probability, and minimise spillages.

The iterative method worked as follows. At the beginning of each iteration, the first model estimated the values of the above-mentioned functions by simulating the refill rule curves existing in the current iteration. Then, the second model produced new rule curves and chose the best ones by optimisation and comparison with the prior values. Finally, to end the iteration, the third model tested the obtained rule curve in order to ensure the safety of the system.

The optimisation model, based on genetic algorithms and progressive optimisation algorithm, considered the influence of the head on power generation. It had a planning period of 6,426 days, corresponding to all refill periods of the whole historical series of water inflows, divided into daily steps and it used the observed data.

- [Wang and Zhang (2012)]

This paper described a short-term model of a decision support tool developed for Central China Grid Company Limited and its application to the cascade system composed of the Three Gorges reservoir and its immediately downstream plant in the Yangtze River (China). The model, based on mixed LP and continuous (from the point of view of the state space) DP, had a planning period of one day divided into hourly steps and its objective was to maximise the revenue of a system for supplying energy and spinning reserve. It took into account the operating states and forbidden zones of the hydro units, which were grouped into equivalent units in order to reduce the computational burden. The water values used were predetermined by sensitivity analysis in a five-daily optimal system operation problem.

\subsubsection{Common absences of the cited models relevant for this work}

All the references about the models that have been discussed in this section have in common two absences which, because of its importance for this thesis, are highlighted below:

1. No estimation of the water values taking into account the effects of $\varphi$ and $\rho$ on the long-term operation has been carried out and, therefore, no sensitivity analysis of the water values to these constraints has been conducted. 
2. No analytical expression of the economic impact that $\varphi$ and $\rho$ would introduce on the operation of a plant of given characteristics has been proposed. 



\section{ASSESING THE LONG-TERM EFFECTS OF MINIMUM FLOWS AND MAXIMUM RAMPING RATES ON HYDROPEAKING}

This chapter is devoted to the achievement of the objectives 3 and 4 of the thesis (as discussed in sec. 1.3) and contains three studies. The first two studies [Guisández et al. (2013) and Guisández et al. (2014)] were mainly conducted for the development of a methodology suitable to assess the long-term economic impact of $\varphi$ and $\rho$ on the operation of a hydropower plant. The third study [Guisández et al. (2016a)] was fully focused on the achievement of the objectives 3 and 4 of the thesis.

The chapter is divided in three parts, one for each study. Each part starts with a description of the methodology applied in every research. Then the case studies considered in the research are presented. Finally, the main results obtained in the study together with their discussion are commented.

\subsection{Assessment of the economic impact of environmental constraints on annual hydropower plant operation}

The main objective of the study which gives name to this section is to propose a methodology for the assessment of the long-term economic impact of $\varphi$ and $\rho$ on the operation of a hydropower plant. The methodology might be of help in a hypothetical negotiation between a hydropower producer and the corresponding river authority about the implementation of a new environmental regime.

\subsubsection{Methodology}

In order to facilitate the understanding of the results and make them applicable to other negotiation processes, both the economic impact caused by the presence of $\varphi$ and $\rho$ and the magnitude of these constraints are expressed in relative terms with respect to the plant under study. Thus, the economic losses are encouraged to be expressed as percentage of the long-term revenue corresponding to the scenario without $\varphi$ and $\rho, \varphi$ as the percentage of the maximum flow of the plant, and $\rho$ as the number of hours necessary for the hydropower plant to "go" from standstill to maximum flow (or vice versa) at a rate equal to the average of the up and down $\rho$. 
The application of the methodology proposed in this study may involve the analysis of a large number of combinations of $\varphi$ and $\rho$, each defined in a long-term optimisation problem, which entails a considerable computational burden. This is the reason why this methodology is deterministic (as discussed in sec. 1.2), follows a solution strategy that takes advantage of the likenesses between the considered problems and uses several models specifically designed for this purpose.

Water inflow and energy price must be considered as input variables. The former is suggested to be modelled by using five annual scenarios (very dry, dry, normal, wet and very wet) with the same probability of occurrence (as in Stillwater Sciences et al. (2006)) and the latter by using the average annual scenario of the historical series. The initial and final $\mathrm{V}$ of all problems must be identical and equal to the most frequent value of the historical series in order to make the calculations easier and more realistic.

Relating to the solution strategy, it is based on the hypothesis that for a given scenario of water inflows and energy prices and for given values of the $\varphi$, the more similar are the $\rho$, the closer to each other will be the optimal policies that should follow the hydropower plant throughout the time of study. The hypothesis was empirically verified in this work.

As regards the models, the proposed methodology is composed of three ones. Sequentially applied by the solution strategy (Fig. 3.1), these models are mainly based on: backward deterministic discrete DP (mod1stu1), deterministic mixed LP (mod3stu1) and a hybrid combination of both optimisation techniques (mod2stu1). These techniques (as discussed in sec. 2.2) have been chosen since DP allows decomposing large problems (in this study, annual problems) into smaller ones (in this study, weekly subproblems), thereby reducing the computational burden, whereas mixed LP offers the opportunity to represent the hydropower operation of a plant with enough accuracy. Finally, it is recommended that the methodology follows the combined optimisation-simulation approach (as discussed in sec. 2.1.1) by recalculating the power outputs and the resulting revenue from the decisions obtained in all LP optimisations with the real generation characteristic of the plant.

\section{- Description of mod1stu1}

The purpose of mod1stu1, Eqs. (A.1, A.5 and A.9-A.10), is to localise a corridor within the space of feasible policies (policy space) close to the optimal policy. Based on backward deterministic discrete DP and limited to problems without $\rho$, mod1stu1 is aimed at maximising the hydropower plant revenue in the day-ahead electricity market throughout a planning period of one year divided into weekly time steps.

mod1stu1 uses $\mathrm{V}$ at the end of each week as decision variable, weekly water inflow and weekly average energy price as input variables, and $\mathrm{V}$ at the beginning of each week as state variable. This study recommends that the discretisation of the policy space should be selected uniformly distributed in such a way that at least three possible $\mathrm{V}$ at the end of every week could be reached from each $\mathrm{V}$ at the beginning of the same week, trying to cover, whenever possible, the full range between the minimum and maximum allowed $\mathrm{V}$. 


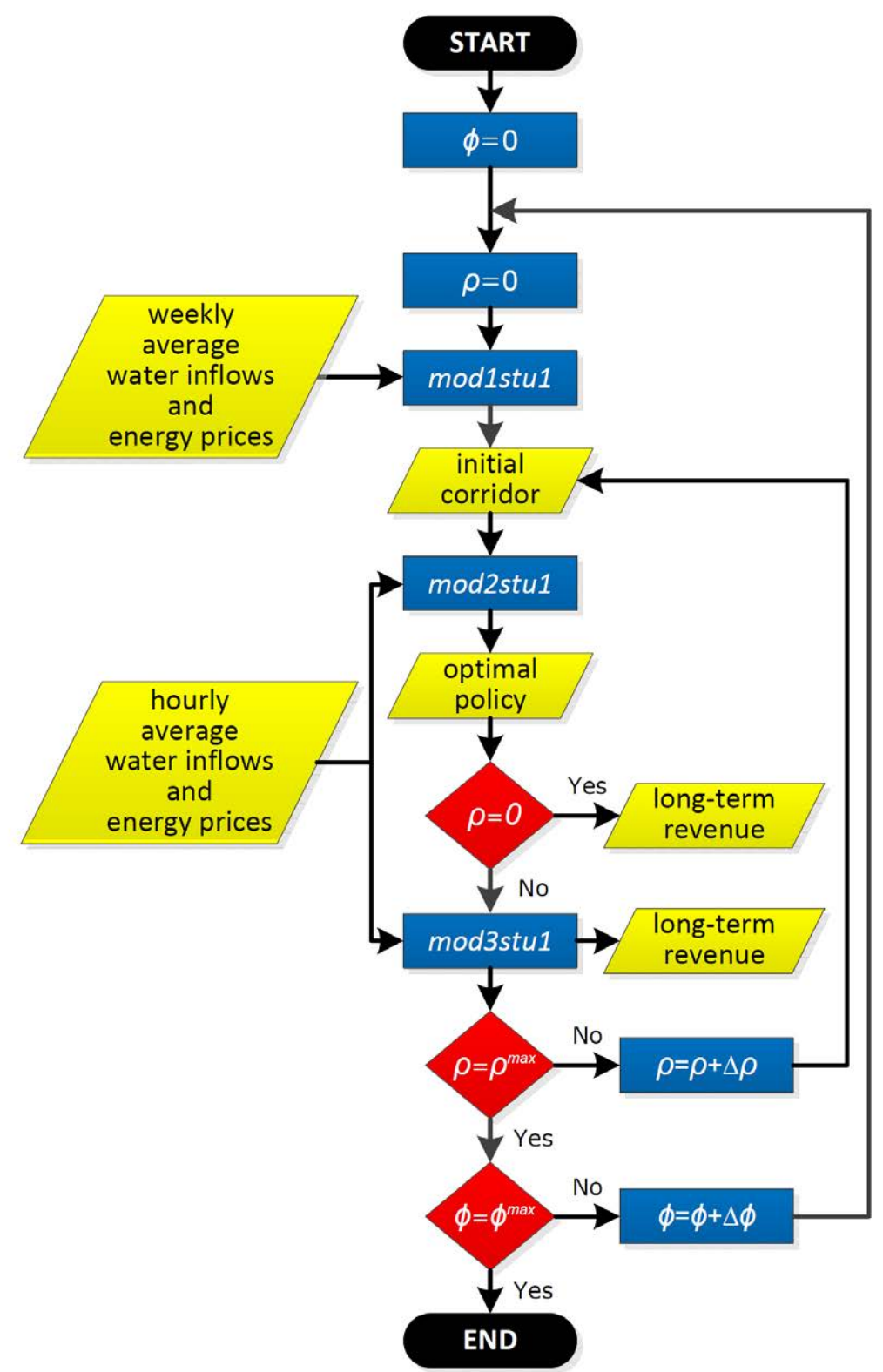

Figure 3.1.: Flowchart of the solution strategy of the study 1 in each scenario.

The revenue corresponding to each feasible transition within the policy space is assumed by mod1stu1 to be proportional to the product of the weekly average energy price, the mean gross head of the transition and the volume released through the hydro units during the transition, Eq. (A.9). This volume is in turn assumed to be equal to the maximum allowed one of the plant, when the average flow released by the reservoir is equal or above the maximum plant flow, or reduced proportionally in other case, Eq. (A.10).

\section{- Description of mod2stu1}

The purpose of mod2stu1, Eqs. (A.1, A.5, A.16, A.21, A.23-A.24, A.27, A.30-A.31, A.46, A.49, A.51-A.60, A.63-A.68 and A.72), is to determine the best policy within the corridor obtained by mod1stu1. It is a hybrid optimisation model consists of two modules, one based 
on backward deterministic discrete DP (DP module) and the other on deterministic mixed LP (LP module).

The process of mod2stu1 is as follows. First, the DP module decomposes the considered annual problem into the weekly subproblems of the policy space contained within the corridor. Then, the LP module optimises the decisions of these subproblems. Next, the DP module recalculates, with the real generation characteristic of the plant, the power outputs and the resulting revenue according to these decisions and obtains their corresponding revenue. Finally, the DP module determines the best policy within the corridor and calculates its corresponding total revenue.

The DP module, Eqs. (A.1 and A.5), is aimed at maximising the hydropower plant revenue in the day-ahead electricity market throughout a planning period of one year divided into weekly time steps. It uses $\mathrm{V}$ at the end of each week as decision variable and $\mathrm{V}$ at the beginning of each week as state variable.

The LP module, Eqs. (A.16, A.21, A.23-A.24, A.27, A.30-A.31, A.46, A.49, A.51-A.60, A.63-A.68 and A.72), is aimed at maximising the hydropower plant revenue in the day-ahead electricity market throughout a planning period of one week divided into hourly time steps. It uses F during each hour as decision variable and hourly water inflow and energy price as input variables.

The objective function of the LP module, Eq. (A.16), is subject to the following constraints: $\varphi$, Eq. (A.21); up and down p, Eqs. (A.23-A.24); water mass balance, Eq. (A.27); initial and final V, Eqs. (A.30-A.31); maximum legal storage capacity of the reservoir, Eq. (A.46); minimum technical storage capacity of the reservoir, Eq. (A.49); flow released through the hydro units, Eq. (A.51); plant generation characteristic model of several concave curves, Eqs. (A.52-A.60); inter-hourly variation of the generated power, Eq. (A.63); start-up and shut-down of the hydro units, Eqs. (A.64-A.68); and maximum flow through the bottom outlets, Eq. (A.72).

\section{- Description of mod3stu1}

The purpose of mod3stu1, Eqs. (A.16, A.21, A.23-A.24, A.27, A.30-A.31, A.33-A.34, A.46, A.49, A.51-A.60, A.63-A.68 and A.72), is to ensure the fulfilment of $\rho$ between two consecutive weeks. Based mainly on deterministic mixed LP and limited to problems with $\rho$, mod3stu1 is practically identical to the LP module of mod2stu1 except the inclusion of two coupling constraints that force the flow released by the plant during the first hour of each week to be equal to that of the last hour of the previous week, Eqs. (A.33-A.34). This heuristic idea (see in Fig. 3.2) will be hereinafter referred to as refinement. As in mod2stu1, mod3stu1 recalculates, with the real generation characteristic of the plant, the power outputs and the resulting revenue from the decisions obtained in all LP optimisations. 
3.1 Assessment of the economic impact of environmental constraints on annual hydropower plant operation

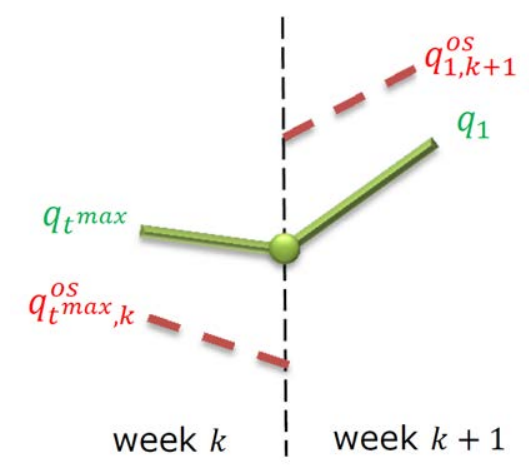

Figure 3.2.: Coupling of the flows released by the plant between consecutive stages.

\subsubsection{Case study}

A Spanish peak hydropower plant (plant I), located in the northwest of Spain (Fig. B.1), was selected to test the above-described methodology. The technical data of the plant were provided by the company that owns and manages it. Tab. B.1 summarises the main design parameters of the plant. The performance curves of the plant, calculated without hydraulic losses, and their linear concave approximations (used by the LP module of mod2stu1 and mod3stu1) are plotted in Fig. B.5(a-b-d). The head-storage curve of the plant is drawn in Fig. B.18(a).

Regarding the considered input variables, on the one hand, the historical series of water inflows were taken from the web page of the Centre for Public Works Studies and Experimentation [CEDEX (2013)]. A linear variation between the mid-days of each two consecutive days was assumed in order to transform the available data (daily flows) into hourly water inflows; the obtained histogram, probability distribution and scenarios can be seen in Fig. B.2. On the other hand, the historical series of hourly energy prices were taken from the web page of the Iberian Electricity Market Operator [OMIE (2011)]; the hourly values and their weekly averages of the used energy prices are depicted in Fig. B.3. The analysed scenarios of these input variables as well as the magnitudes of $\varphi$ and $\rho$ considered in the study are included in Tab. 3.1; the maximum values of $\varphi$ and $\rho$ were taken from the web page of the Miño-Sil River Basin District Authority [CHMS (2010)].

Table 3.1.: Input variable scenarios and environmental constraints used in the study 1 .

\begin{tabular}{|c|c|c|}
\hline CONCEPT & SCEnARIOS & DESCRIPTION \\
\hline \hline \multirow{2}{*}{ Water inflows } & $\begin{array}{c}\text { Very dry; Dry; } \\
\text { Normal; Wet; Very wet }\end{array}$ & $\begin{array}{c}\text { Equiprobable } \\
\text { and hourly }\end{array}$ \\
\hline Energy prices & Average & Hourly \\
\hline \multirow{2}{*}{$\varphi$} & $0.0 \% ; 0.6 \% ; 1.2 \% ; 1.8 \% ;$ & $\varphi_{\text {Oct. }- \text { Dec. }}=0.75 \varphi ; \varphi_{\text {Jan. }- \text { Mar. }}=1.75 \varphi ;$ \\
& $2.4 \% ; 3.0 \% ; 4.0 \% ; 5.0 \%$ & $\varphi_{\text {Apr. }- \text { Jun. }}=1.20 \varphi ; \varphi_{\text {Jul. }- \text { Sep. }}=0.30 \varphi$ \\
\hline$\rho$ & $0 \mathrm{~h} ; 6 \mathrm{~h} ; 12 \mathrm{~h} ;$ & $\rho_{1-k^{\text {max }}}^{\text {up }}=0.75 \rho ;$ \\
$\rho_{1-k^{\text {max }}}=1.50 \rho$
\end{tabular}


Following the above-mentioned discretisation criterion (as discussed in sec. 3.1.1), the state diagram of the problem comprises 11 states per stage. It results in a total of 280 annual problems to be assessed ( 5 water inflow scenarios $\cdot 1$ energy price scenario $\cdot 56$ combinations of $\varphi$ and $\rho$ ), i.e. a total of 275,765 weekly subproblems to be solved. As a result of mod1stu1 (39,031 subproblems), only a third of the total had to be solved by mod2stu1 (91,360 subproblems), of which less than $14 \%$ had to be recalculated by mod3stu1 (12,480 subproblems). Thus, 280 problems decomposed in a total of 142,871 subproblems were solved.

The proposed methodology (as discussed in sec.3.1.1) was implemented in MATLAB ${ }^{\circledR}$, each subproblem being solved using CPLEX ${ }^{\mathrm{TM}}$ under GAMS ${ }^{\mathrm{TM}}$, on two threads in parallel [Baslis and Bakirtzis (2011)] of an Intel ${ }^{\circledR}$ Core $^{\mathrm{TM}}$ i5 at $2.4 \mathrm{GHz}$ and 4 GB RAM running on Microsoft Windows ${ }^{\circledR}$. As recommended in Carvalho and Pinto (2006) (seen in sec. 2.2.1), a sensitivity analysis of the performance of the above-described models to certain parameters (Tab. 3.2) was carried out. After this analysis, the most time consuming problem and its counterpart without $\varphi$ and $\rho$ were calculated with practically no time limit $\left(10^{9} \mathrm{~s}\right)$ and a very demanding relative optimality criterion $(0.1 \%$ ) in order to check the accuracy of the solutions obtained with the chosen values of these parameters (Tab. 3.2). The error between the economic impacts calculated by means of the two approaches was only $1.8 \%$.

Table 3.2.: Characteristic parameters of each model of the study 1.

\begin{tabular}{|c|c|c|}
\hline Models & PARAMETER & VALUE \\
\hline \hline mod1stu1 & Radius of the corridor (\% useful volume) & 40 \\
\hline \multirow{4}{*}{ mod2stu1 } & Maximum number of performance curves $\left(\overline{c^{\max }}\right)$ & $\infty$ \\
\cline { 2 - 3 } & Gross head "covered" by each performance curve $\left(H^{\%}\right)$ & 20 \\
\cline { 2 - 3 } & Relative optimality criterion $(\%)$ & 2 \\
\cline { 2 - 3 } mod3stu1 & Time limit for each subproblem $(\mathrm{s})$ & 1,800 \\
\cline { 2 - 3 } & Maximum number of performance curves $\left(\overline{c^{\max }}\right)$ & $\infty$ \\
\cline { 2 - 3 } & Gross head "covered" by each performance curve $\left(H^{\%}\right)$ & 10 \\
\cline { 2 - 3 } & Relative optimality criterion $(\%)$ & 1,800 \\
\hline
\end{tabular}

\subsubsection{Main results and discussion}

Fig. 3.3(a) represents the level curves of the average annual losses for different values of $\varphi$ and $\rho$. This figure reveals, among other things, that the combination proposed by the river basin authority for this plant, given by $\varphi=5 \%$ and $\rho=60 \mathrm{~h}$, would reduce the plant revenues more than $10 \%$. Regarding the joint influence of $\varphi$ and $\rho$, it is clearly appreciable that the higher the one, the smaller the rise in the economic impact caused by the increase in the other. Fig. 3.3(b-c-d-e-f) represent in turn the same level curves of each water inflow scenario. As it can be observed in these figures, the smaller the rainfall of the scenario, the greater were the annual revenue losses caused by a given combination of $\varphi$ and $\rho$. 
(a)

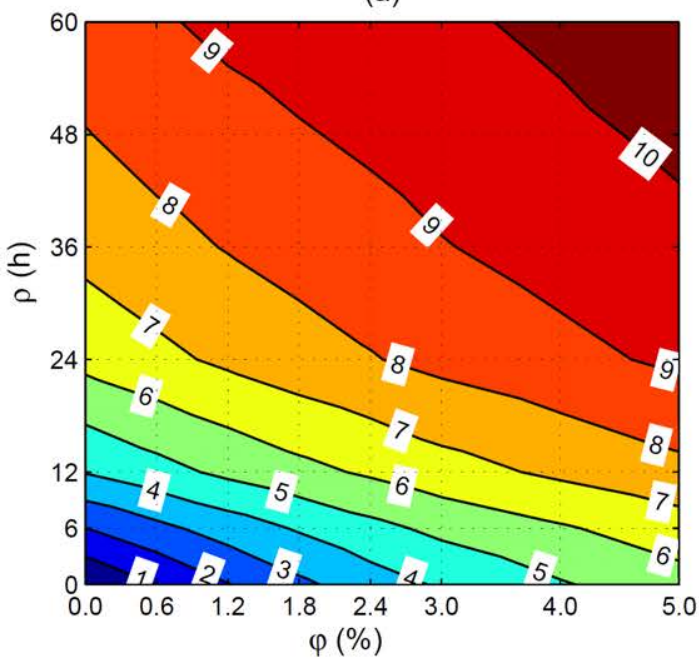

(c)

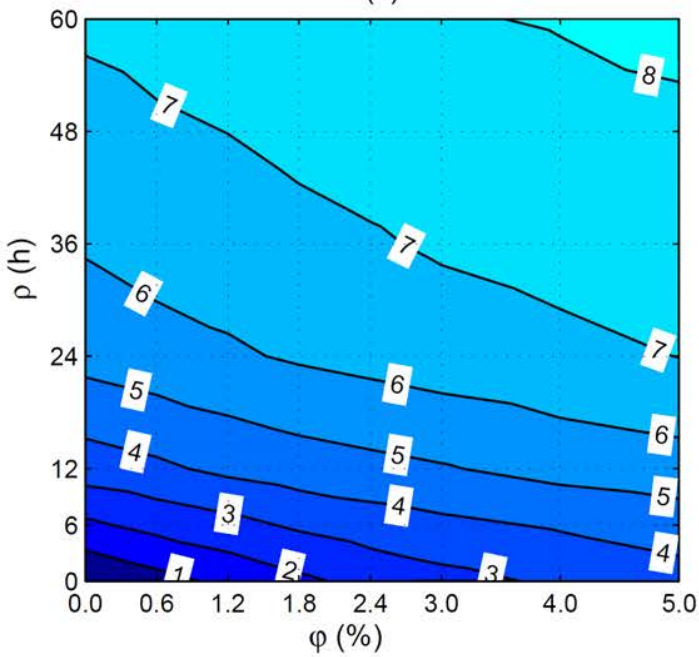

(e)

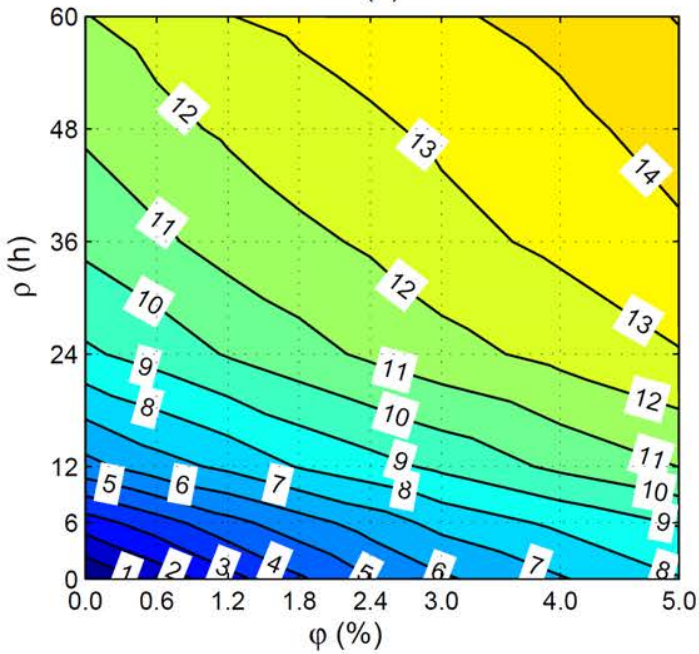

(b)

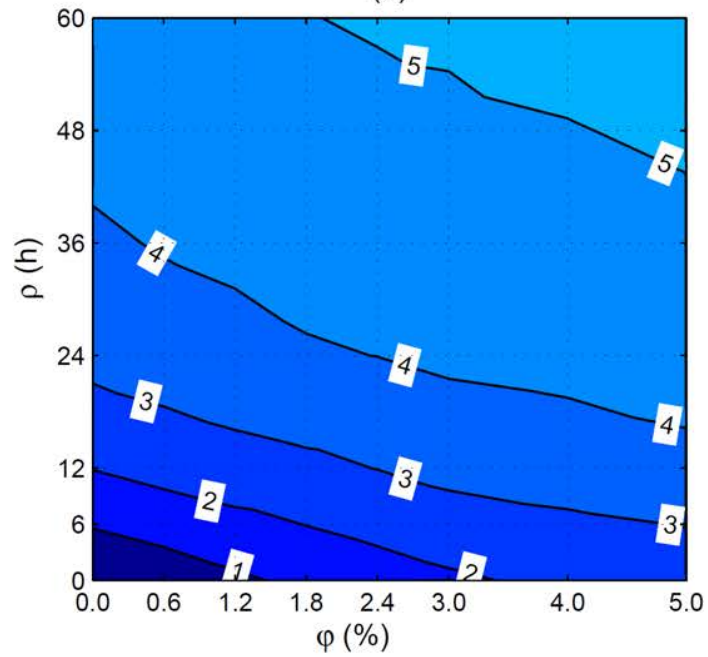

(d)

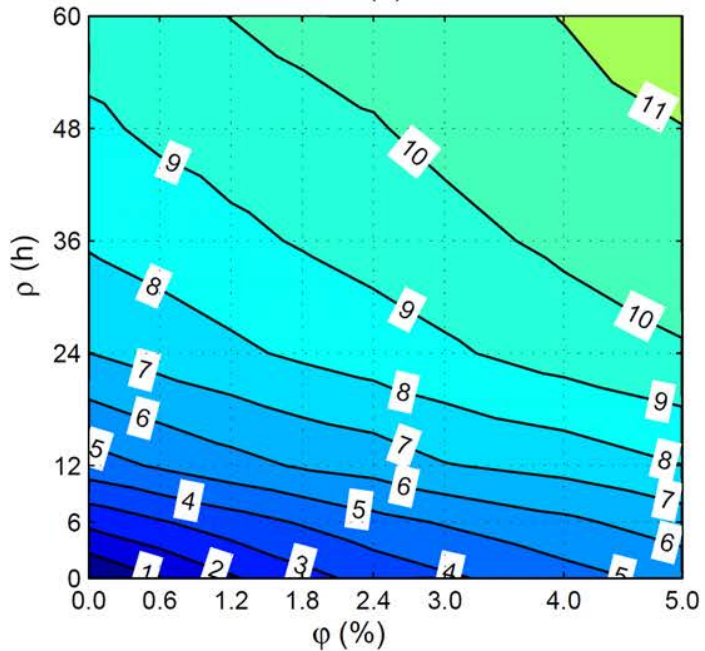

(f)

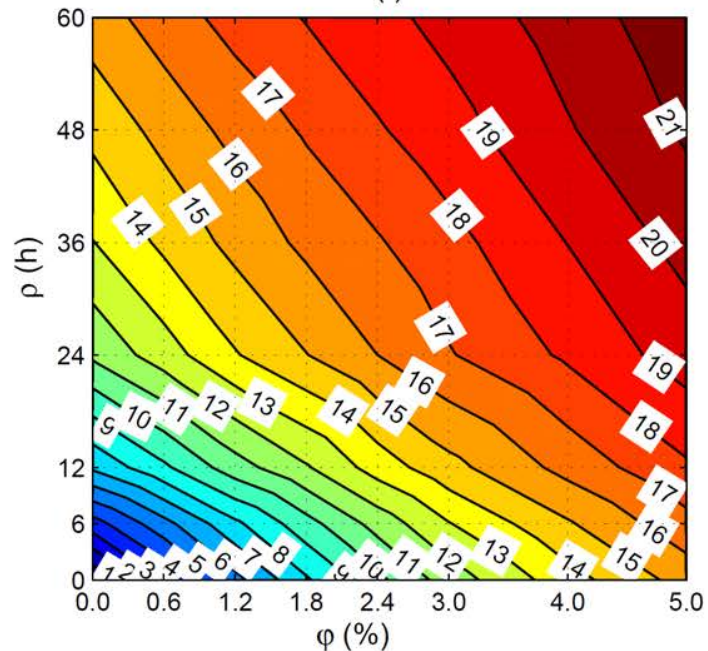

Figure 3.3.: Annual losses according to $\varphi$ and $\rho$ of the plant I corresponding to the: (a) Average of the water years; b) Very wet water year; c) Wet water year; d) Normal water year; e) Dry water year; f) Very dry water year scenario.

The evolution of the average annual losses as a function of $\varphi$ and $\rho$ are depicted in Fig. 3.4(ab), respectively. As it can be seen, for a given $\varphi$, the annual revenue losses followed a quadratic 
behaviour as $\rho$ varied; whereas for a given $\rho$, the annual losses followed an almost linear behaviour as $\varphi$ varied. Similar trends were obtained in Chen and Forsyth (2008), Olivares (2008), Niu and Insley (2010), Pérez-Díaz and Wilhelmi (2010), Pérez-Díaz et al. (2012) and Rheinheimer et al. (2013) (as discussed in sec. 2.5).
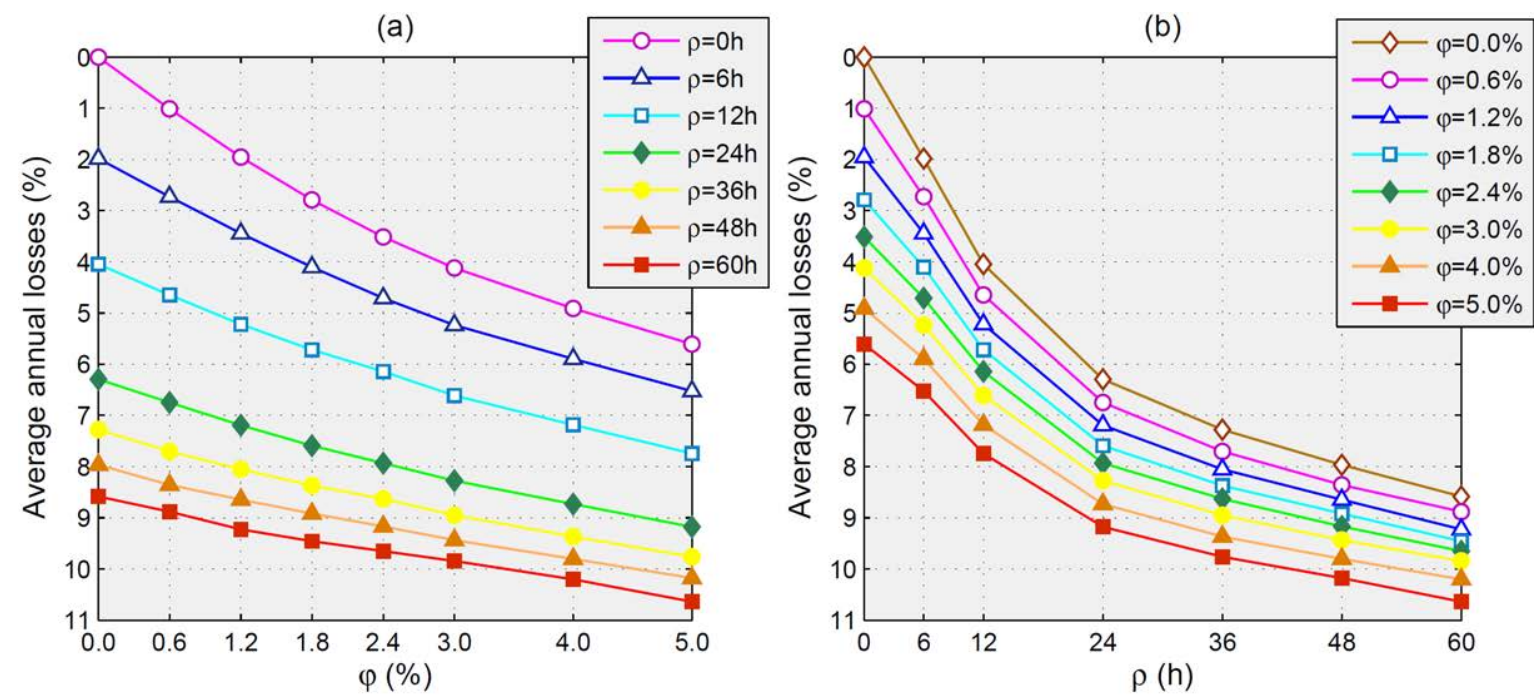

Figure 3.4.: Average annual losses of the plant I according to: (a) $\varphi$; (b) $\rho$.

In Fig. 3.5, the histogram of the initial and final $\mathrm{F}$ of the subproblems of the best policies with $\rho>0$ obtained by mod2stu1 is drawn. It can be appreciated that, despite its irregularity, the distribution covered practically the whole range of possible values. It is significant that three quarters of the values were grouped around $\varphi$ and $q^{2}$. It is also interesting to note how certain flows standed out above the rest; these in order of frequency were: $\varphi(46.0 \%)$, $q^{2}(10.2 \%), Q^{\min }(6.5 \%), Q^{\max }(6.5 \%), q^{3}(4.2 \%), 66 \mathrm{~m}^{3} / \mathrm{s}$ that was the flow corresponding to the maximum efficiency for one hydro unit $(3.5 \%)$, and $198 \mathrm{~m}^{3} / \mathrm{s}$ that was the one corresponding to the maximum efficiency for the whole plant $(2.0 \%)$.

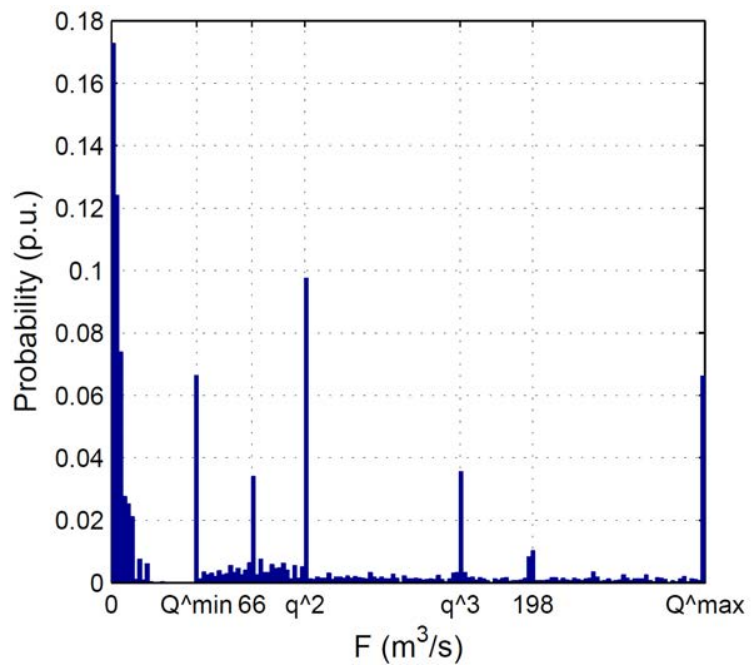

Figure 3.5.: Histogram of the initial and final $\mathrm{F}$ of the subproblems of the best policies with p>0 obtained by mod2stu1. 
3.2 Effects of the maximum flow ramping rates on the long-term operation decisions of a hydropower plant

Finally, all the above-mentioned breaches of $\rho$ between the last hour of each week and the first hour of the following one were computed and it was observed that the average length of these breaches was above five hours with a deviation standard of more than ten hours. Therefore, the heuristic idea of the applied refinement (illustrated in Fig. 3.2) might be accurate enough for the proposed methodology. Nevertheless, given the magnitude of these breaches, it would be convenient to develope new ways to couple the hydropower operation between consecutive stages; e.g., to add a new state variable in the DP that ensures the fulfilment of $\rho$ along the whole planning period.

\subsection{Effects of the maximum flow ramping rates on the long-term operation decisions of a hydropower plant}

The main objective of the study of the current section is to analyse the influence of $\rho$ on the annual operation of a real peak hydropower plant. The secondary objective is to test whether the introduction of $\mathrm{F}$ as a second state variable into the DP-based long-term scheduling can improve the performance of the model developed so far.

\subsubsection{Methodology}

Five annual models were used in the study 2. The models are mainly based on: backward deterministic discrete DP (mod1stu2), deterministic mixed LP (mod3Vstu2 and mod3VFstu2) and a hybrid combination of backward incremental DP and deterministic mixed LP (mod2 Vstu2 and mod2VFstu2). The reason why the methodology applied in this study moved from the simplest discrete DP (used in the previous study) to the incremental variant of DP is due to the computational burden increases exponentially with the number of state variables in DP (as discussed in sec. 2.2.2) and the selected deterministic approach allows this change (as discussed in sec.1.2). On the other hand, this methodology still follows the combined optimisation-simulation approach (as discussed in sec. 2.1.1) by recalculating the power outputs and the resulting revenue from the decisions obtained in all LP optimisations with the real generation characteristic of the plant.

As regards the used incremental DP, the chosen convergence criterion combined the two criteria normally used with this technique (as discussed in sec. 2.2.2). If the obtained policy "touched" any border of the corridor, a new corridor was built around said policy with the previous discretisation size; otherwise, the discretisation size of the corridor was reduced. The process stopped, defining the optimal policy, once that the difference in annual revenue between consecutive iterations was lower than the selected tolerance: in this study was $0.1 \%$ [Nandalal and Bogardi (2007)].

The developed models considered again (as in the study 1) water inflow and energy price as input variables but both variables were modelled by using the average annual scenario of their respective historical series. The initial and final $\mathrm{V}$ of all problems were also specified 
with the same value, equal to the most frequent value of the historical series.

Due to the test nature of the study and given that it was focused specifically on $\rho$, only two environmental situations were analysed: without and with $\rho$. Thus, the used solution strategy, although simpler than the previous one, was still taking advantage of the likenesses between the three considered annual problems. These problems, listed in order of resolution, were: problem without $\rho$, problem with $\rho$ considering $\mathrm{V}$ as state variable, and problem with $\rho$ considering $\mathrm{V}$ and $\mathrm{F}$ as state variables.

\section{- Description of mod1stu2}

The purpose of mod1stu2, Eqs. (A.1, A.5 and A.10-A.12), is the same as that of mod1stu1 (as discussed in sec.3.1.1). It is an improved version of that model and it is also based on backward deterministic discrete DP. The main difference between them is how the revenue corresponding to each feasible transition within the policy space is obtained. In mod1stu2, the weekly revenue is estimated as the product of the weekly average energy price, the generated power (determined from the average net head and the plant operating flow) and the operating time, Eq. (A.12). This time is assumed to be equal to 168 hours, when the average flow released by the reservoir is equal or above the operational minimum, or reduced proportionally in other case, Eq. (A.11). Finally, given the few problems to solve, the discretisation of the policy space used by mod1stu2 was finer than in the previous study, reaching $1 \%$ of $\mathrm{V}$.

- Description of mod2Vstu2

The purpose of mod2Vstu2, Eqs. (A.1, A.5, A.13, A.16, A.21, A.23-A.24, A.27, A.30-A.31, A.46, A.49, A.51-A.60, A.63-A.68 and A.72), is the same as that of mod2stu1 (as discussed in sec. 3.1.1). As that model, mod2Vstu2 is a hybrid optimisation model, but instead of on backward deterministic discrete DP, is based on backward incremental DP. Therefore, it is an improved version of mod2stu1 whose only difference from that model is the need of verify a convergence criterion related to its incremental nature, Eq. (A.13).

\section{- Description of mod2VFstu2}

The purpose of mod2VFstu2, Eqs. (A.3, A.5, A.7, A.13, А.16, А.21, А.23-A.24, A.27, A.30-A.31, A.35-A.36, A.46, A.49, A.51-A.60, A.63-A.68 and A.72), is the same as that of mod2Vstu2 and its structure is practically identical to that model. The main novelty of mod2VFstu2 is the use of $\mathrm{F}$ as state variable.

On the one hand, the DP module needs a new state transition equation, Eq. (A.7), that represents the operational inertia of the reservoir, and, therefore, to replace the one-dimensional DP equation, Eq. (A.1), by its two-dimensional equivalent, Eq. (A.3). On the other hand, the LP module includes a pair of constraints that considers the initial and final F, Eqs. (A.35-A.36), defined by the DP module and involved in that new state transition equation, Eq. (A.7). 
3.2 Effects of the maximum flow ramping rates on the long-term operation decisions of a hydropower plant

\section{- Description of mod3Vstu2}

The main purpose of mod3Vstu2, Eqs. (A.16, A.21, A.23-A.24, A.27, A.30-A.31, A.33-A.34, A.46, A.49, A.51-A.58, A.61-A.68 and A.72), is the same as that of mod3stu1 (as discussed in sec. 3.1.1) and its secondary purpose is to improve the revenue. Based mainly on deterministic mixed LP, mod3Vstu2 refines as in the study 1, Eqs. (A.33-A.34), the policy obtained by mod2Vstu2 (illustrated in Fig. 3.2). Moreover, it is an improved version of mod3stu1 because the generation characteristic model is shaped non-concave, Eqs. (A.61-A.62).

\section{- Description of mod3VFstu2}

The purpose of mod3VFstu2, Eqs. (A.16, A.21, A.23-A.24, A.27, A.30-A.31, A.35-A.36, A.46, A.49, A.51-A.58, A.61-A.68 and A.72), is to recalculate the policy obtained by mod2VFstu2 with a non-concave generation characteristic model, Eqs. (A.61-A.62), in order to obtain the same accuracy as mod3Vstu2. Based mainly on deterministic mixed LP and very similar to that model, mod3VFstu2 replaces the refinement constraints, Eqs. (A.33-A.34), by another pair, Eqs. (A.35-A.36), with the aim of considering the initial and final $\mathrm{F}$ of each subproblem defined by mod2VFstu2.

\subsubsection{Case study}

Plant I (Tab. B.1), the same peak hydropower plant as in the study 1, located in the northwest of Spain (Fig. B.1), was the selected one for carrying out the current study. The performance curves of the plant, calculated without hydraulic losses, and both their linear concave (used by mod2Vstu2 and mod2VFstu2) and non-concave (used by mod3Vstu2 and mod3VFstu2) approximations, are plotted in Fig. B.5. The head-storage curve of the plant is drawn in Fig. B.18(a).

Regarding the considered input variables, the same historical series applied in the study 1 were used [CEDEX (2013); OMIE (2011)]. Their scenarios as well as the magnitudes of $\varphi$ and $\rho$ considered in this study are included in Tab. 3.3; the values of $\varphi$ and $\rho$, expressed in relative terms following the methodology of the study 1 (as discussed in sec. 3.1.1), were those planned to be imposed by the water basin authority of the studied plant [CHMS (2010)].

Table 3.3.: Input variable scenarios and environmental constraints used in the study 2 .

\begin{tabular}{|c|c|c|}
\hline CONCEPT & SCENARIOS & DESCRIPTION \\
\hline \hline Water inflows & Average & Hourly \\
\hline Energy prices & Average & Hourly \\
\hline$\varphi$ & $5.0 \%$ & $\begin{array}{c}\varphi_{\text {Oct. }- \text { Dec. }}=0.75 \varphi ; \varphi_{\text {Jan. }- \text { Mar. }}=1.75 \varphi ; \\
\varphi_{\text {Apr.-Jun. }}=1.20 \varphi ; \varphi_{\text {Jul.-Sep. }}=0.30 \varphi\end{array}$ \\
\hline$\rho$ & $0 \mathrm{~h} ; 60 \mathrm{~h}$ & $\rho_{1-k_{\text {max }}}^{\text {up }}=0.75 \rho ; \rho_{1-k^{\max }}^{\text {down }}=1.50 \rho$ \\
\hline
\end{tabular}

For the resolution procedure of the problem without $\rho: 109,463$ subproblems were calculated by mod1stu2, 3,494 by mod2Vstu2 and 52 by mod3Vstu2. For the procedure of the problem 
with $\rho$ considering $\mathrm{V}$ as state variable: 3,874 subproblems were calculated by mod2Vstu2 and 52 by mod3Vstu2. And for the third resolution procedure: 8,656 subproblems were calculated by mod2VFstu2 and 52 by mod3VFstu2. Therefore, 3 problems decomposed in a total of 125,643 subproblems were solved in this study.

The above-described optimisation models (as discussed in sec. 3.2.1) were implemented in MATLAB $^{\circledR}$, each subproblem being solved using CPLEX $^{\mathrm{TM}}$ under GAMS $^{\mathrm{TM}}$, on four threads in parallel [Baslis and Bakirtzis (2011)] of an Intel ${ }^{\circledR}$ Core $^{\mathrm{TM}}$ i5 at $2.4 \mathrm{GHz}$ and 4 GB RAM running on Microsoft Windows ${ }^{\circledR}$. Again, following the suggestion in Carvalho and Pinto (2006), a sensitivity analysis of the performance of the models involved in the last part of the resolution procedures to certain parameters (Tab. 3.4) was carried out. Half a thousand randomly selected [MacCallum et al. (1999)] weekly subproblems were calculated with practically no time limit $\left(10^{9} \mathrm{~s}\right)$ and a very demanding relative optimality criterion $(0.1 \%)$ in order to check the accuracy of the solutions obtained with the chosen values of these parameters (Tab. 3.4). The error between the economic impacts calculated by means of the two approaches was only $4.8 \%$.

Table 3.4.: Characteristic parameters of the LP algorithms of the study 2.

\begin{tabular}{|c|c|}
\hline PARAMETER & VALUE \\
\hline \hline Maximum number of performance curves $\left(\overline{c^{\max }}\right)$ & $\infty$ \\
\hline Gross head "covered" by each performance curve $\left(H^{\%}\right)$ & 10 \\
\hline Relative optimality criterion $(\%)$ & $0.1^{a}$ \\
\hline Time limit for each subproblem (s) & 10 \\
\hline
\end{tabular}

${ }^{a}$ As recommended in Fu and Shahidehpour (2007)

\subsubsection{Main results and discussion}

The main results of the optimal policies of the plant I considered in the study 2 are summarised in Tab.3.5. As it can be observed, on the one hand, the economic impact of $\rho$ was almost $6 \%$ even though the energy generation was more than $2 \%$ greater and the number of hours in operation was almost $20 \%$ greater. Some of these effects were obtained in Edwards et al. (1999), Harpman (1999), Kotchen et al. (2006), Chen and Forsyth (2008), Olivares (2008), Niu and Insley (2010), Pérez-Díaz and Wilhelmi (2010), Babel et al. (2012), Pérez-Díaz et al. (2012), Niu and Insley (2013) and Rheinheimer et al. (2013) (as discussed in sec. 2.5). On the other hand, Tab. 3.5 shows that the average units' efficiency was $1.1 \%$ higher than the one without $\rho$, and the efficiency standard deviation was $1.3 \%$ lower than the one obtained without $\rho$, what yielded a more efficient operation. Furthermore with $\rho$, the number of start-ups and shut-downs of the hydro units was about $90 \%$ lower and the total volume discharged by the bottom outlets was significantly lower, about one half. Finally, regarding the introduction of $\mathrm{F}$ as a second state variable into the incremental DP, it can also be noted that the improvement in the annual revenue was barely appreciable, only $0.2 \%$. 
3.3 Approximate formulae for the assessment of the long-term economic impact of environmental constraints on hydropeaking

Table 3.5.: Main values of the optimal policies of the plant I considered in the study 2 .

\begin{tabular}{|c|c|c|c|c|c|c|}
\hline $\begin{array}{c}\text { OPtIMAL } \\
\text { POLICY OF } \\
\text { THE PROBLEM }\end{array}$ & $\begin{array}{c}\text { ANNUAL } \\
\text { REVENUE } \\
{[\mathrm{M} \in]}\end{array}$ & $\begin{array}{c}\text { GENERATED } \\
\text { ENERGY } \\
{[\mathrm{GWh}]}\end{array}$ & $\begin{array}{c}\text { OPERATING } \\
\text { HOURS } \\
{[\mathrm{h}]}\end{array}$ & $\begin{array}{c}\text { AVERAGE } \\
\text { EFFICIENCY } \\
{[\%]}\end{array}$ & $\begin{array}{c}\text { START-UP } \\
\text { AND } \\
\text { SHUT-DOWNS }\end{array}$ & $\begin{array}{c}\text { WATER } \\
\text { SPILLAGES } \\
{[\mathrm{hm} 3]}\end{array}$ \\
\hline \hline without $\rho$ & 45.28 & 910.5 & 6,175 & $90.3 \pm 2.5$ & 1,248 & 64.8 \\
\hline with $\rho$ by V & 42.59 & 933.5 & 7,394 & $91.4 \pm 1.2$ & 110 & 33.9 \\
\hline with $\rho$ by V\&F & 42.72 & 936.0 & 7,384 & $91.4 \pm 1.2$ & 96 & 33.3 \\
\hline
\end{tabular}

\subsection{Approximate formulae for the assessment of the long-term economic impact of environmental constraints on hydropeaking}

The objectives of the present study correspond to the objectives 3 and 4 of the thesis (as discussed in sec.1.3). The former is defined by the title of the current section and the latter is aimed to provide insight into the effects of $\varphi$ and $\rho$ on hydropeaking.

\subsubsection{Methodology}

Given the complexity of the studied phenomenon (as discussed in sec. 1.1) and, therefore, the inherent difficulty to deduce its exact analytical expression, several regression models were developed to reach the desired formulae. In order to obtain these formulae, a sample, as large and representative as possible, of results of long-term hydro scheduling problems subject to different magnitudes of $\varphi$ and $\rho$ corresponding to distinct hydropower plants was used. Thus, a very much larger computational burden than in the study 1 was faced and therefore, as before, similar measures were again taken.

Once more, the approach was deterministic and it only used water inflow and energy price as input variables. Therefore, the study should at least be considered useful for clarifying the effects of the main terms involved in the obtained formulae [Rossi (2012)]. Only one scenario per hydropower plant was calculated (the average historical values of the input variables) in order to reduce even more the computational burden than in the study 1 . With the aim of greater realism, as in the previous studies, the initial and final $\mathrm{V}$ of all problems were also specified with the same value, equal to the most frequent value of the historical series of each considered reservoir.

Once more also, the solution strategy was based on the same hypothesis made in the study 1 (as discussed in sec. 3.1.1) but now, taking advantage of what has been learned later, the three sequentially used optimisation models (see in Fig. 3.6) are based on the same techniques applied in the study 2: backward deterministic discrete DP (mod1stu2; as discussed in sec. 3.2.1 but in this study with a discretisation of the policy space of 33 values [Bogardi et al. (1988)]), deterministic mixed LP (mod3stu3) and a hybrid combination of backward incremental DP and deterministic mixed LP (mod2stu3). Because of different 
hydropower plants were evaluated, the planning period used for each one varied between one or more years, as a function of the reservoir's regulation capacity. As both previous studies, the methodology follows the combined optimisation-simulation approach (as discussed in sec. 2.1.1) by recalculating the power outputs and the resulting revenue from the decisions obtained in all LP optimisations with the real generation characteristic of the considered plant.

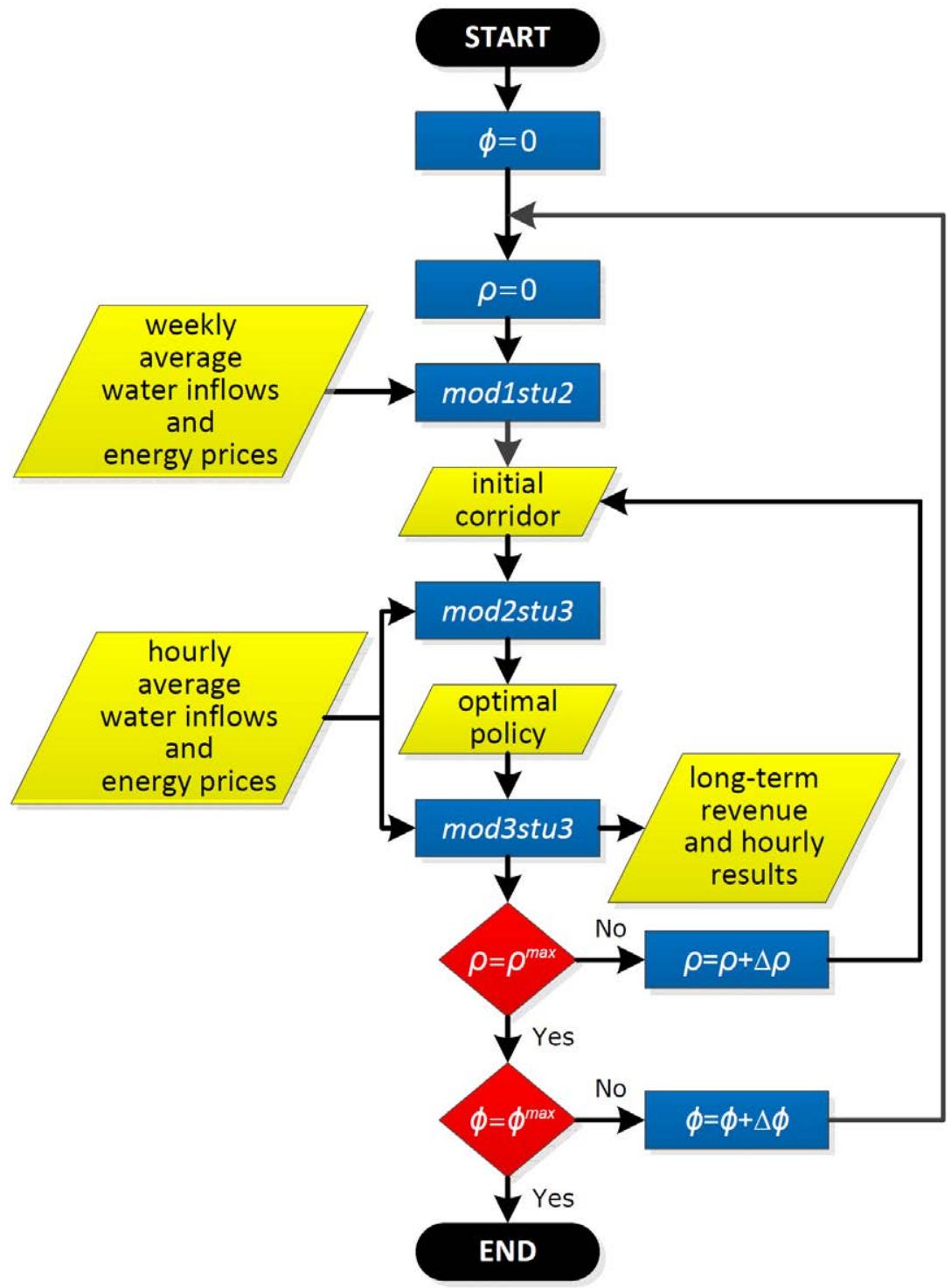

Figure 3.6.: Flowchart of the solution strategy of the study 3 in each hydropower plant.

Regarding to the development of the regression models, the first efforts to get them were based on correlation and factor analysis but, given the resulting low accuracy, this procedure was abandoned in favour of the classical trial-and-error approach. The results of the factor analysis helped to orient the trial-and-error based search. However, hundreds of attempts were made to reach the final formulae showed in this study. Finally, three formulae are provided since two of them are focused on problems in which only $\varphi$ or $\rho$ are present, and therefore are univariate, and the third one, bivariate, is for the problems with both constraints. 
3.3 Approximate formulae for the assessment of the long-term economic impact of environmental constraints on hydropeaking

\section{- Description of mod2stu3}

The purpose of mod2stu3, Eqs. (A.3, A.5, A.7, A.13, A.16, A.21, A.23-A.24, A.27, A.30-A.31, A.37-A.39, A.46, A.49, A.51-A.60, A.63-A.68 and A.72), is the same as that of mod2VFstu2 (as discussed in sec.3.2.1) but its procedure is slightly different from that model.

Initially, because of the findings of the study 2 (as discussed in sec. 3.2.3), the current study was going to use the refinement proposed in the study 1 (illustrated in Fig. 3.1.1). However, during the course of this study, the first results that were obtained demonstrated that that approach would be generally valid only for low values of $\rho$, that is, approximately below 24 hours.

Given the necessity of using both state variables and taking advantage of the lower influence of $\mathrm{F}$ on the revenue, mod2stu3 considers both variables but $\mathrm{V}$ is treated in an incremental manner (alike in an incremental DP), whereas F is coarsely discretised. In this way, the applied corridors decrease only in one dimension, corresponding to $\mathrm{V}$, as the solution search advances. Thus, V starts with 33 values [Bogardi et al. (1988)] and, in each iteration, is doubled, while the dimension of $\mathrm{F}$ is maintained in four plus the number of hydro units of the considered plant. Based on the results of the study 2 (as discussed in sec. 3.2.3), these flows correspond to $\varphi, Q^{\text {min }}$, their average, $Q^{\text {max }}$ and as many flows as hydro units installed in the plant, these equidistributed between $Q^{\min }$ and $Q^{\max }$.

Finally, the LP module of mod2stu3 shows a small modification of the initial and final $\mathrm{F}$ constraints of the hydro units, Eqs. (A.35-A.36), in order to improve the performance during the first hour of each subproblem and to fulfil $\rho$ in every hour, Eqs. (A.37-A.39).

\section{- Description of mod3stu3}

The main purpose of mod3stu3, Eqs. (A.16-A.18, A.21, A.23-A.24, A.27, A.30-A.31, A.37A.39, A.42-A.46, A.49, A.51-A.58, A.61-A.68 and A.72), is to reduce the errors caused by the discretisation of the state variables in the policy obtained by mod2stu3. Moreover, mod3stu3 has as secondary purpose the same of mod3VFstu2 (as discussed in sec. 3.2.1).

In order to achieve its main purpose, mod3stu3 undertakes successive relaxations of the state boundary conditions at the end of each subproblem. Thus, from the policies obtained by mod2VFstu3, a series of 2-weekly, or longer in the case of hydropower plants with multiannual reservoirs, subproblems are solved with an overlap of one week between consecutive subproblems. The final V and F constraints, Eqs. (A.31 and A.39), used in the previous models are relaxed, Eqs. (A.42-A.45), and a penalty term is included in the objective function as a function of the final $\mathrm{V}$ and of this one and the final F, Eqs. (A.17 or A.18). Then, but now week by week, without overlap and by reintroducing the final $\mathrm{V}$ and $\mathrm{F}$ constraints, Eqs. (A.31 and A.39), the resulting policy is calculated for the last time by mod3stu3 and its hourly results are saved. 


\subsubsection{Case studies}

A dozen of peak hydropower plants, located in several regions of Spain (Fig. B.1), were selected to perform this study. There were two main criteria for choosing these hydropower plants. First, every hydropower plant must be located at the head of its basin with the aim of ignoring the influence of the rest of the water basin regulation infrastructures, without a significant loss of accuracy as well as of easing the characterisation of the water inflows to its reservoir. Second, every hydropower plant must also show, as far as possible, different characteristics with respect to the other hydropower plants in order to increase the diversity and representativity of the sample.

Tab. B.3 includes the main design parameters of the selected hydropower plants. The results in terms of long-term economic impacts corresponding to the hydropower plants (I-X) in this table were used to derive the formulae whereas the results corresponding to the other hydropower plants (A and B) were used to check their accuracy. The performance curves of the plants, calculated without hydraulic losses, and both their linear concave (used by mod2stu3) and non-concave (used by mod3stu3) approximations, are plotted in Fig. B.5 to Fig. B.17. The head-storage curves of the plants are drawn in Fig. B.18 and Fig. B.19.

Regarding the historical series of the considered input variables, the hourly energy prices (see in Fig. B.3) were taken from the web page of the Iberian Electricity Market Operator [OMIE (2011)] and the monthly water inflows (see in Fig. B.20 and Fig. B.21) from the web pages of the Centre for Public Works Studies and Experimentation [CEDEX (2013)] and of the Catalan Water Agency [ACA (2015)]. The analysed scenarios of these input variables as well as the magnitudes of $\varphi$ and $\rho$ evaluated in the study, expressed in relative terms following the methodology of the study 1 (as discussed in sec. 3.1.1), are included in Tab. 3.6.

The above-described optimisation models (as discussed in sec. 3.3.1) were implemented in MATLAB ${ }^{\circledR}$, each subproblem being solved using CPLEX $^{\mathrm{TM}}$ under GAMS ${ }^{\mathrm{TM}}$, on eight computers, totaling 52 threads (used each pair in parallel [Baslis and Bakirtzis (2011)] at each computer): one Intel ${ }^{\circledR} \mathrm{Xeon}^{\circledR}$ E5 at $1.8 \mathrm{GHz}$ and 16 GB RAM, two Intel ${ }^{\circledR}$ Core $^{\mathrm{TM}}$ i3 at $2.13 \mathrm{GHz}$ and $4 \mathrm{~GB}$ RAM, two Intel ${ }^{\circledR} \mathrm{Core}^{\mathrm{TM}}$ i5 at $2.4 \mathrm{GHz}$ and 4 GB RAM, two Intel ${ }^{\circledR}$ Core $^{\mathrm{TM}}$ i7 at $2.4 \mathrm{GHz}$ and $8 \mathrm{~GB}$ RAM, and one Intel ${ }^{\circledR} \mathrm{Xeon}^{\circledR}$ E5 at $3.1 \mathrm{GHz}$ and $64 \mathrm{~GB}$ RAM. Once more, following the suggestion in Carvalho and Pinto (2006), a small sensitivity analysis of the performance of the mixed LP algorithms was undertaken, using as a basis the results of that carried out in the study 2 (as discussed in sec. 3.2.2). The resulting selected parameters are given in Tab. 3.7.

A total of 574 long-term problems were solved in this study; 476 to derive the formulae and 98 to check their accuracy. These problems were decomposed into 14,072,632 short-term subproblems: 712,552 were solved by mod1stu2, 12,892,379 by mod2stu3, and 467,701 by mod3stu3. 
3.3 Approximate formulae for the assessment of the long-term economic impact of environmental constraints on hydropeaking

Table 3.6.: Input variable scenarios and environmental constraints used in the study 3 .

\begin{tabular}{|c|c|c|}
\hline CONCEPT & SCENARIOS & DESCRIPTION \\
\hline \hline Water inflows & Average & Hourly \\
\hline Energy prices & Average & Hourly \\
\hline \multirow{2}{*}{$\varphi$} & $0 \% ; 1 \% ; 2 \% ; 3 \% ;$ & $\varphi_{\text {Oct. }- \text { Dec. }}=0.75 \varphi ; \varphi_{\text {Jan }- \text { Mar. }}=1.75 \varphi ;$ \\
& $4 \% ; 5 \% ; 7 \% ; 10 \%$ & $\varphi_{\text {Apr.-Jun. }}=1.20 \varphi ; \varphi_{\text {Jul. }- \text { Sep. }}=0.30 \varphi$ \\
\hline \multirow{2}{*}{$\rho$} & $0 \mathrm{~h} ; 6 \mathrm{~h} ; 12 \mathrm{~h} ;$ & $\rho_{1-k_{\text {max }}^{\text {up }}}^{\text {up }}=0.75 \rho ;$ \\
& $18 \mathrm{~h} ; 24 \mathrm{~h} ; 48 \mathrm{~h} ; 72 \mathrm{~h}$ & $\rho_{1-k^{\max }}^{\text {dow }}=1.50 \rho$ \\
\hline
\end{tabular}

Table 3.7.: Characteristic parameters of the LP algorithms of the study 3.

\begin{tabular}{|c|c|c|c|}
\hline MOdEL & $\begin{array}{l}\text { PlanNing } \\
\text { PERIOD }\end{array}$ & Parameter & Value \\
\hline \multirow{4}{*}{$\begin{array}{c}\text { mod2stu3's } \\
\text { LP } \\
\text { module }\end{array}$} & \multirow{4}{*}{ Weekly } & Maximum number of performance curves $\left(\overline{c^{\max }}\right)$ & $\infty$ \\
\hline & & $\begin{array}{c}\text { Gross head "covered" by each } \\
\text { performance curve }\left(H^{\%}\right)\end{array}$ & 10 \\
\hline & & Relative optimality criterion (\%) & 1.0 \\
\hline & & Time limit for each subproblem $(\mathrm{s})$ & 10 \\
\hline \multirow{8}{*}{ mod3stu3 } & \multirow{4}{*}{ N-weekly } & Maximum number of performance curves $\left(\overline{c^{\max }}\right)$ & 1 \\
\hline & & $\begin{array}{c}\text { Gross head "covered" by each } \\
\text { performance curve }\left(H^{\%}\right)\end{array}$ & 100 \\
\hline & & Relative optimality criterion (\%) & 0.5 \\
\hline & & Time limit for each subproblem (s) & $(\mathrm{N}-1) \cdot 25 \cdot u^{\max }$ \\
\hline & \multirow{4}{*}{ Weekly } & Maximum number of performance curves $\left(\overline{c^{\max }}\right)$ & $\infty$ \\
\hline & & $\begin{array}{c}\text { Gross head "covered" by each } \\
\text { performance curve }\left(H^{\%}\right)\end{array}$ & 10 \\
\hline & & Relative optimality criterion (\%) & $0.1^{a}$ \\
\hline & & Time limit for each subproblem (s) & $100 \cdot u^{\max }$ \\
\hline
\end{tabular}

${ }^{a}$ As recommended in Fu and Shahidehpour (2007)

\subsubsection{Main results and discussion}

Each of the formulae (3.1-3.3) gives a rough estimation of the long-term economic impact on the operation of a peak hydropower plant, expressed as percentage of the revenue corresponding to the scenario without environmental constraints, caused by $\varphi$ and/or $\rho(\varepsilon)$ as a function of two adimensional parameters of the plant: maximum plant flow divided by average river flow $\left(Q^{\text {max }} / W^{a v g}\right)$ and minimum plant flow divided by average river flow $\left(Q^{\text {min }} / W^{a v g}\right)$.

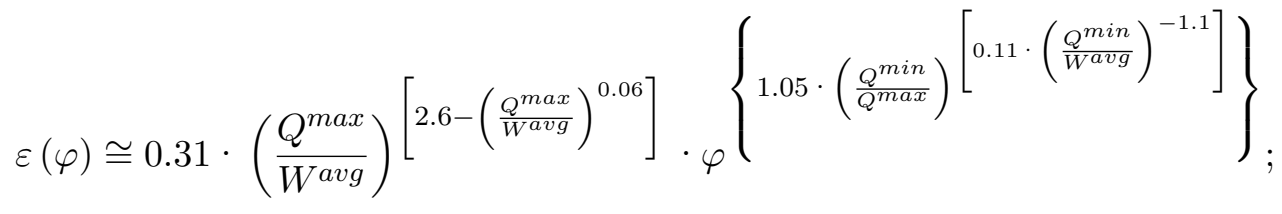

$$
\begin{aligned}
& \forall \varphi \in[0,10] \mid Q^{\max } \cdot \varphi<W^{a v g}
\end{aligned}
$$




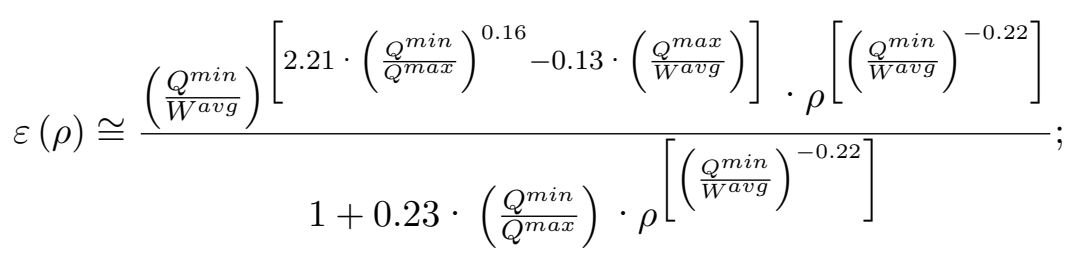

$\forall \rho \in[0,72]$

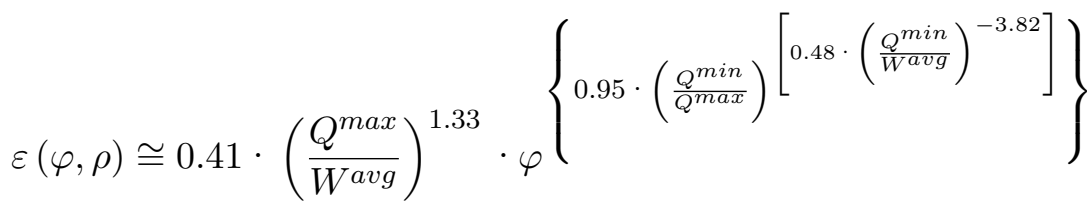

$$
\begin{aligned}
& +\frac{\left(\frac{Q^{\min }}{W^{\text {avg }}}\right)^{\left[1.68-0.16 \cdot\left(\frac{Q^{\max }}{W^{\text {avg }}}\right)\right]} \cdot \rho\left[\left(\frac{Q^{\min }}{W^{\text {avg }}}\right)^{-0.15}\right]}{{ }_{\varphi}\left[\left(\frac{Q^{\max }}{W^{\text {avg }}}\right)^{-0.88}\right]}+0.23 \cdot\left(\frac{Q^{\min }}{Q^{\max }}\right) \cdot \rho^{\left[\left(\frac{Q^{\min }}{W^{\text {avg }}}\right)^{-0.15}\right]} ; \\
& \forall \varphi \in[1,10] \mid Q^{\max } \cdot \varphi<W^{a v g} \wedge \forall \rho \in[6,72]
\end{aligned}
$$

Tab. 3.8 summarises the main statistical parameters of these formulae, as well as their performance for the problems considered with the test plants A and B.

Table 3.8.: Main statistical parameters of the formulae and summary of the results obtained with the test plants.

\begin{tabular}{|c|c|c|c|}
\hline \multirow{2}{*}{ CONCEPT } & \multicolumn{3}{|c|}{ FORMULA } \\
\cline { 2 - 4 } & $(3.1)$ & $(3.2)$ & $(3.3)$ \\
\hline \hline Sample size & 68 & 70 & 348 \\
\hline Average relative error of the sample (\%) & 8.2 & 7.5 & 8.1 \\
\hline Standard deviation of the sample (\%) & 9.0 & 8.6 & 7.2 \\
\hline Maximum relative error at 95\% confidence interval (\%) & 19.0 & 17.6 & 17.3 \\
\hline Number of problems checked of the hydropower plant A & 7 & 7 & 36 \\
\hline Average relative error of the predicted values for A (\%) & 2.4 & 6.9 & 4.5 \\
\hline Standard deviation of the predicted values for A (\%) & 2.4 & 4.3 & 4.7 \\
\hline Maximum relative error of the predicted values for A (\%) & 6.5 & 13.2 & 18.8 \\
\hline Number of problems checked of the hydropower plant B & 7 & 7 & 36 \\
\hline Average relative error of the predicted values for B (\%) & 7.9 & 4.6 & 7.4 \\
\hline Standard deviation of the predicted values for B (\%) & 10.4 & 2.1 & 4.8 \\
\hline Maximum relative error of the predicted values for B (\%) & 30.0 & 6.2 & 17.2 \\
\hline
\end{tabular}

The long-term economic impact calculated (by the models) and predicted (by the formulae) in these test plants for different values of $\varphi, \rho$, and $\varphi$ and $\rho$ simultaneously are represented in Fig. 3.7(a-b), Fig. 3.7(c-d) and Fig. 3.8, respectively. As it can be observed, the differences between them were not significant, thus verifying the adequacy of the proposed formulae. 


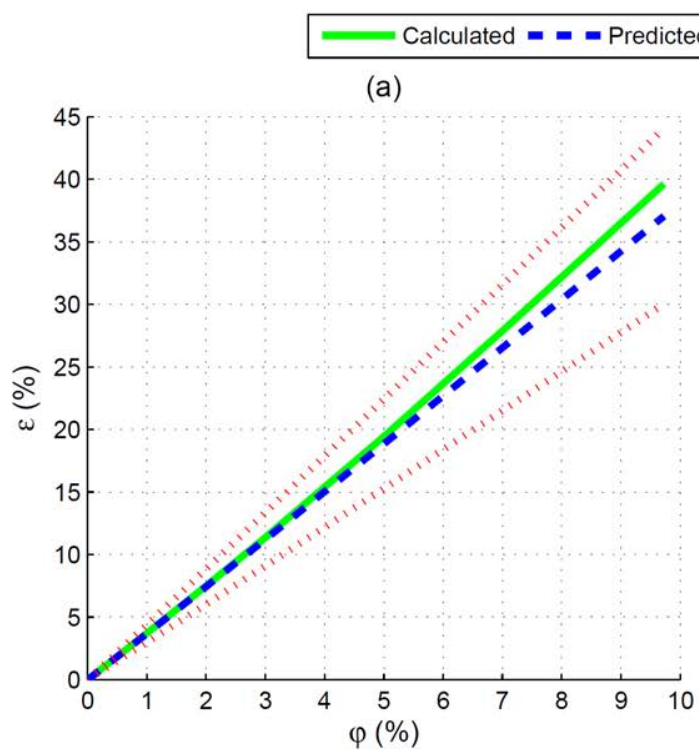

(c)

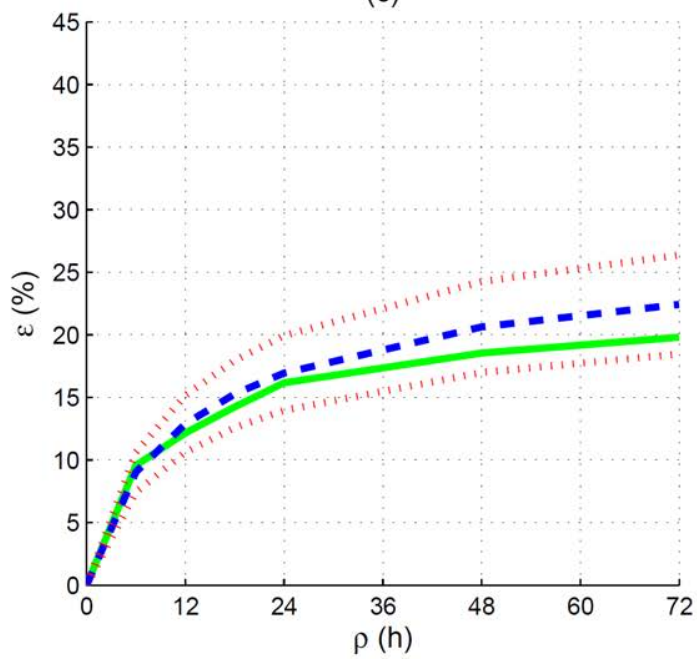

(b)

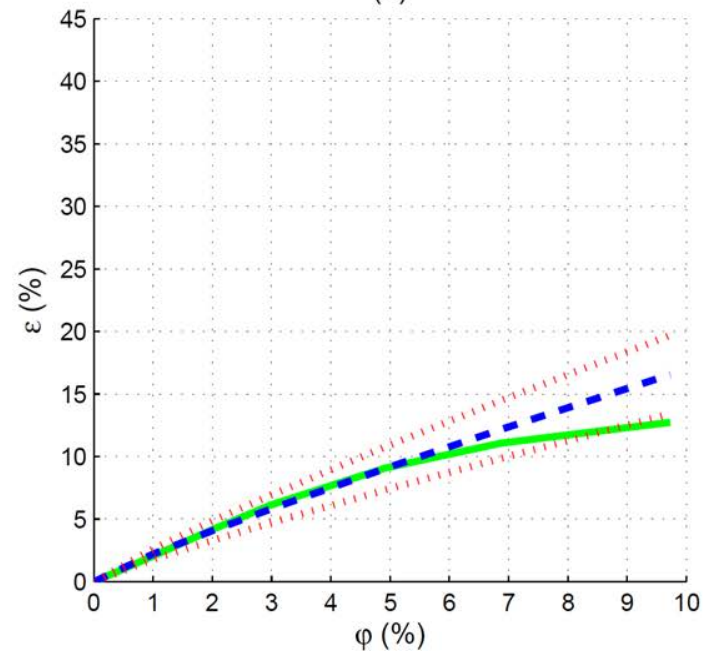

(d)

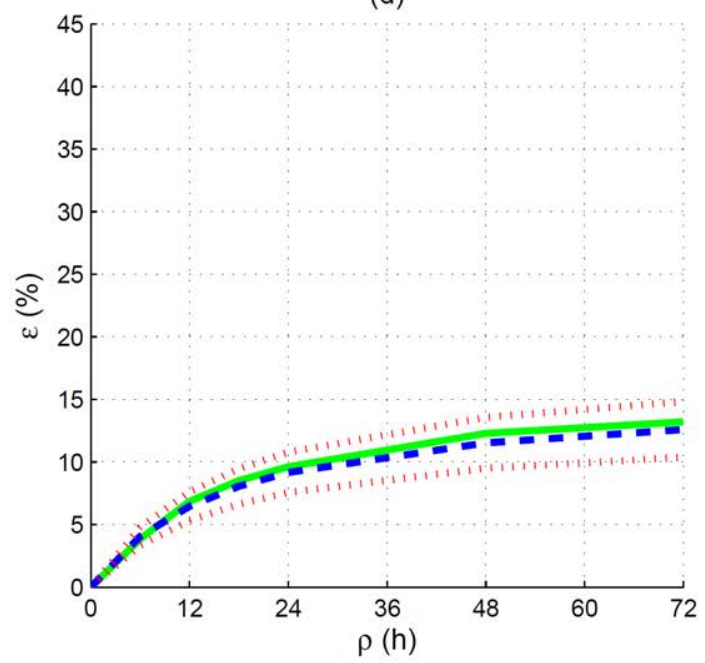

Figure 3.7.: Calculated and predicted long-term economic impact in terms of: (a) $\varphi$ of the plant $\mathrm{A}$; (b) $\varphi$ of the plant $\mathrm{B}$; (c) $\rho$ of the plant $\mathrm{A}$; (d) $\rho$ of the plant $\mathrm{B}$.

A ten of the selected hydropower plants have annual regulation capacity (hereinafter referred to as annual plants). The power - price-duration curves of the set formed by these annual plants (I-VIII, A and B) in terms of $\varphi$ and $\rho$ are depicted in Fig. 3.9, respectively. As it can be expected, the larger $\varphi$ or $\rho$, the worse the price tracking, this effect being more pronounced in the cases subject to $\rho$. Furthermore, Fig. 3.10 shows the energy generated by the annual plants distributed in quartiles of energy prices, in terms of $\varphi$ and $\rho$, respectively (Q1 and Q4 correspond respectively to the higher and lower prices). It is worth noting that, while the amount of generated energy remained almost constant for the second and third quartiles of the energy prices (Q2 and Q3), a progressive transfer of the energy generated at first quartile prices (Q1) to the fourth one (Q4) took place when $\varphi$ or $\rho$ were increased. 
(a)

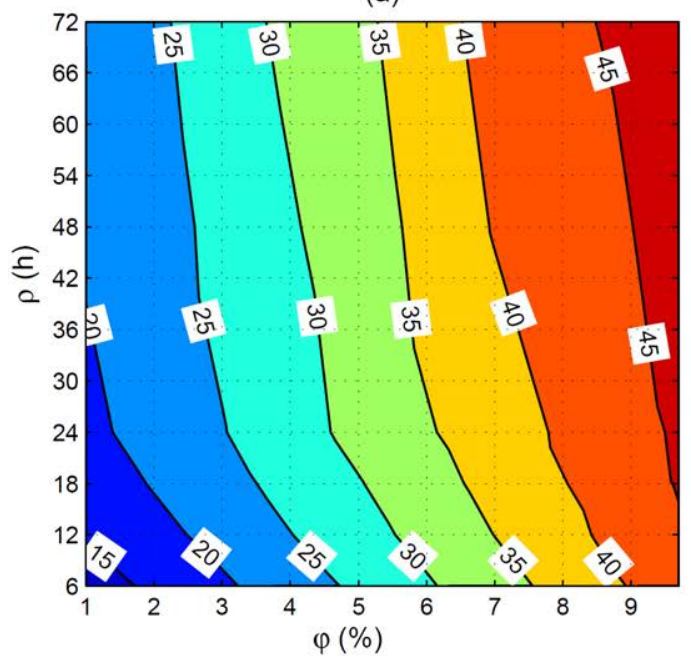

(c)

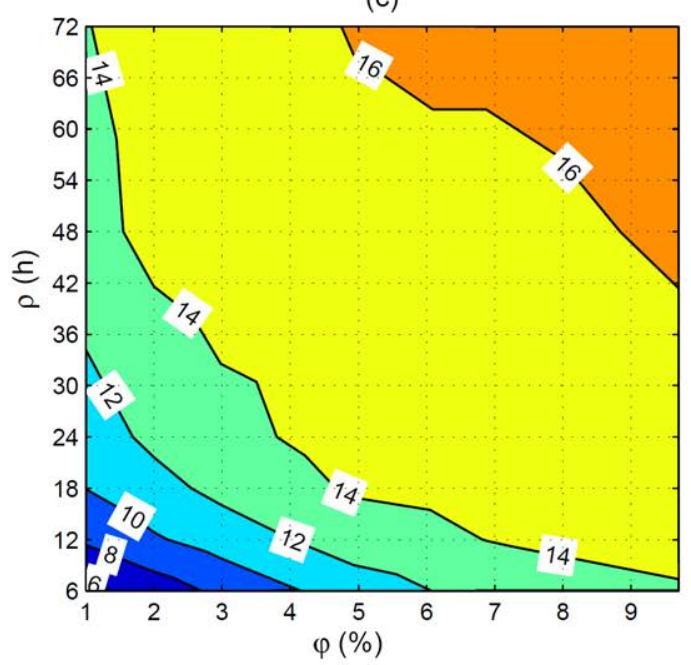

(b)

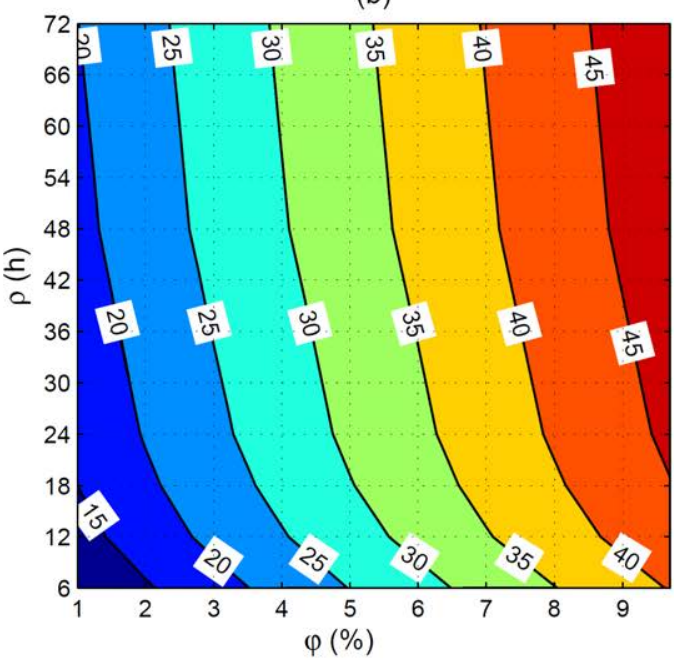

(d)

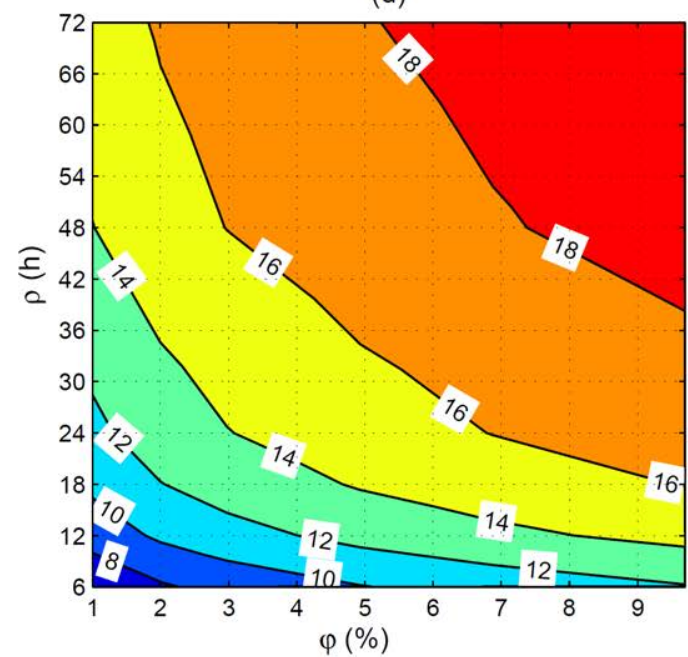

Figure 3.8.: Long-term economic impact in terms of $\varphi$ and $\rho$ of the plant: (a) A calculated by the models; (b) A predicted by formula (3.3); (c) B calculated by the models; (d) B predicted by formula (3.3).

(a)

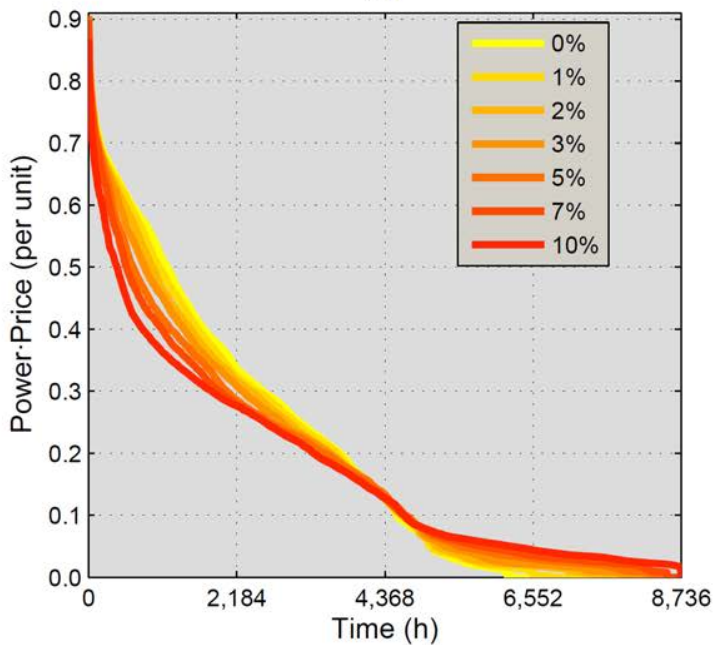

(b)

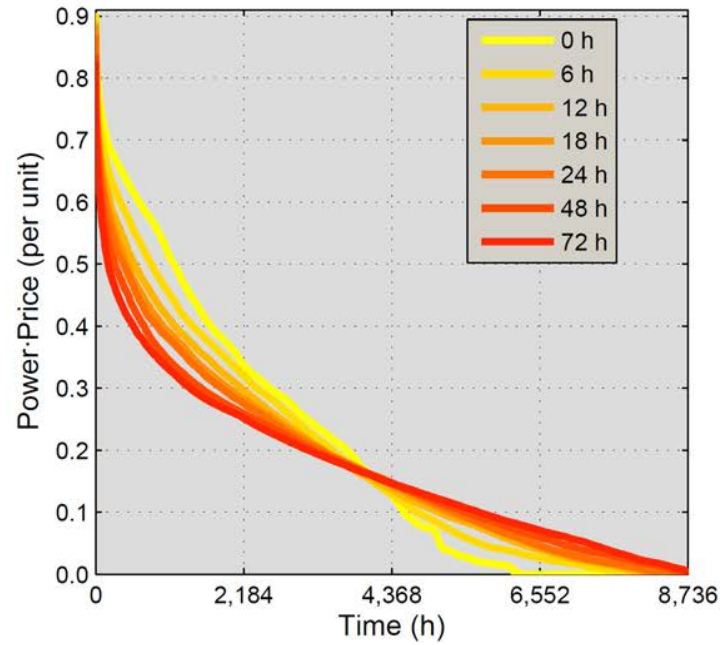

Figure 3.9.: Power · Price-duration curves of the annual plants in terms of: (a) $\varphi$; (b) $\rho$. 
3.3 Approximate formulae for the assessment of the long-term economic impact of environmental constraints on hydropeaking

(a)

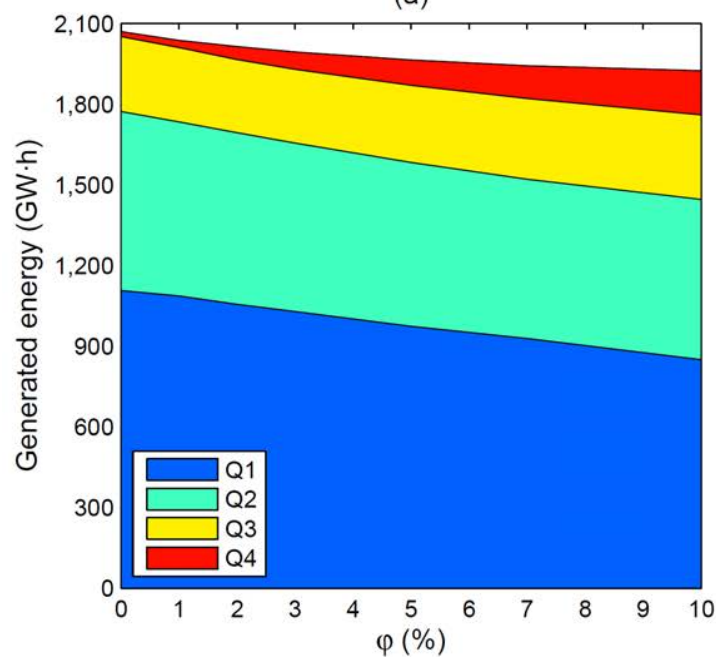

(b)

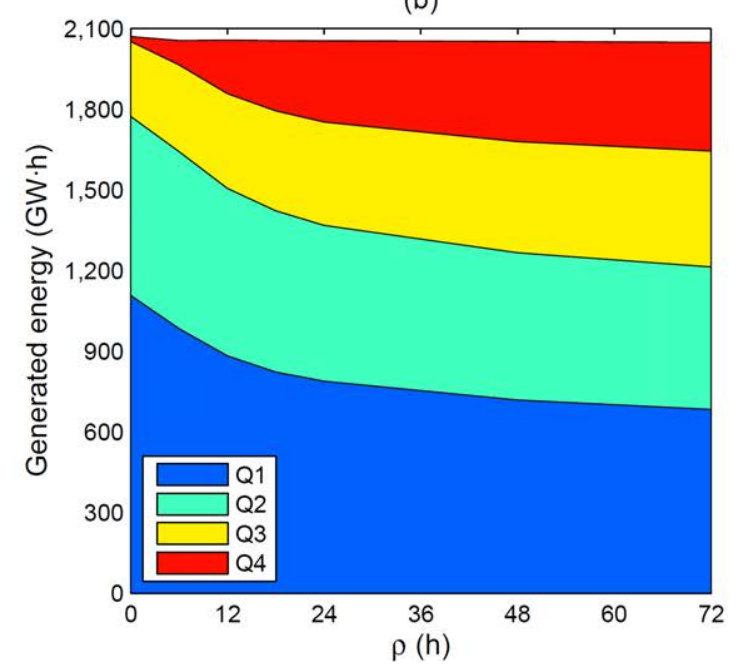

Figure 3.10.: Energy generated by the annual plants in terms of price quartiles and: (a) $\varphi$; (b) $\rho$.

In Fig. 3.11 are drawn the variations relative to the problem without environmental constraints, in terms of $\varphi$ and $\rho$, of the average efficiency, net head, number of operating hours, spillage volumes and start-ups and shut-downs across the annual plants, corresponding to the cases in which only $\varphi$ or $\rho$ are present. As it can be seen both Fig. 3.10(a) and Fig. 3.11(a), as $\varphi$ increased, the number of operating hours and the amount of spilled water grew, while the efficiency, the net head, the total generated energy and the number of start-ups and shut-downs of the hydro units decreased. In the same way in Fig. 3.11(b), as p increased, the operating hours and the spillage volumes grew and the number of start-ups and shut-downs of the hydro units decreased. Some of these effects could be expected to a certain extent; both $\varphi$ and $\rho$ limit the capacity of the hydropower plant to concentrate the water released through the turbines in the peak hours at rated or design flow.

Among the just-mentioned behaviours, only the following were fulfilled in each hydropower plant practically always: as $\varphi$ increased, the spillage grew and the generated energy and the number of start-ups and shut-downs decreased; as $\rho$ increased, the operating hours and spillages grew, whereas the number of start-ups and shut-downs decreased. As it was discussed in sec. 2.5, these effects of $\varphi$ on the spillages and the generated energy as well as the one of $\rho$ on the number of operating hours were obtained in Edwards et al. (1999), Harpman (1999), Kotchen et al. (2006), Chen and Forsyth (2008), Olivares (2008), Niu and Insley (2010), Pérez-Díaz and Wilhelmi (2010), Babel et al. (2012), Pérez-Díaz et al. (2012), Niu and Insley (2013) and Rheinheimer et al. (2013). Regarding the enhancer effect of $\rho$ on the spillages, it seems to be contradictory to the findings of the study 2 (summarised in Tab. 3.5) but it must be remembered that in the two scenarios investigated by that study $\varphi$ was strongly present. The same mitigating effect of $\rho$ on the spillages when $\varphi$ was significant was found in the current study. Moreover, the decrease of the number of start-ups and shut-downs as a consequence of $\rho$, was also detected in the study 2 . Finally, the reduction in the number of 
start-ups and shut-downs caused by $\varphi$ is a contribution of this study.
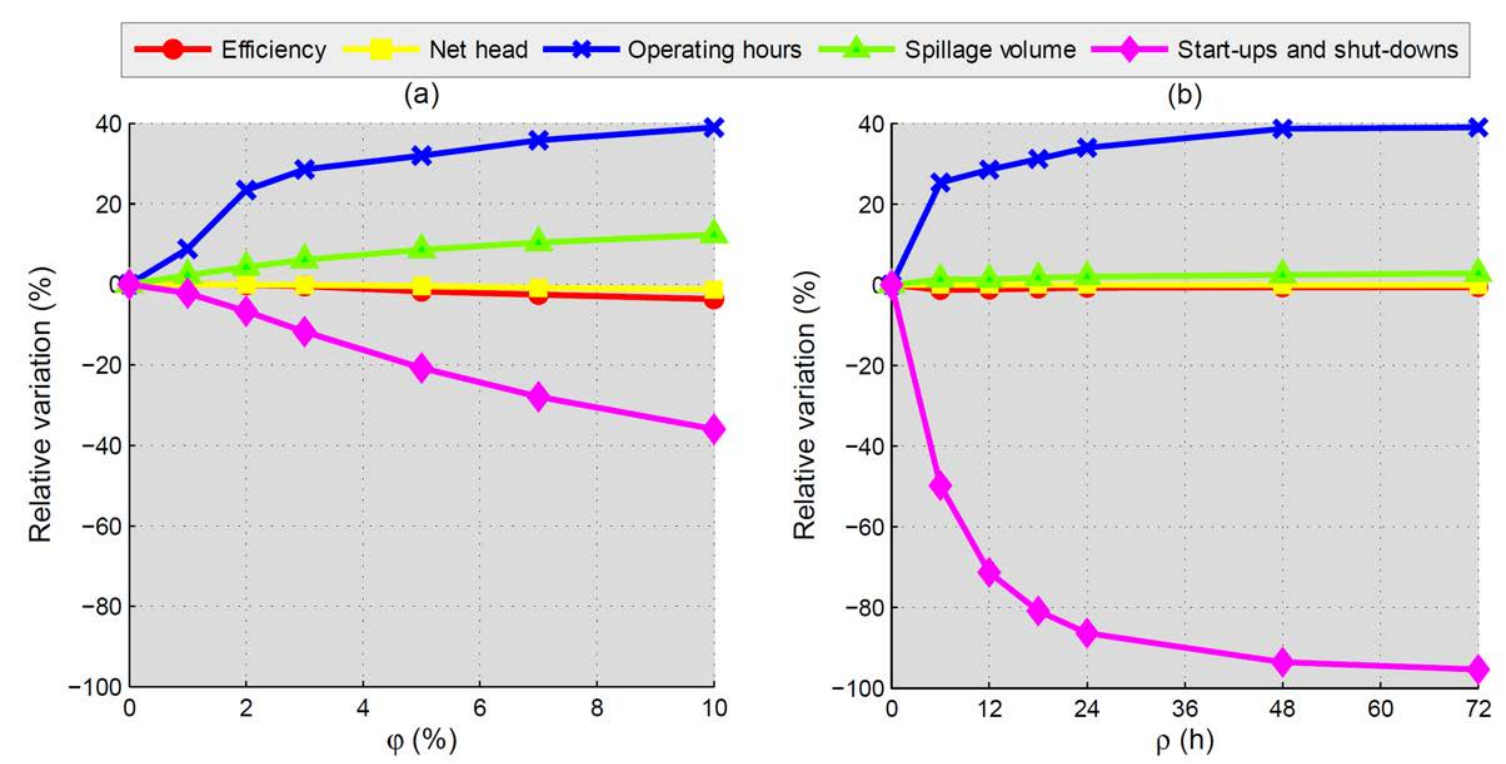

Figure 3.11.: Variations relative to the problem without environmental constraints of the averages of the efficiency, net head, operating hours, spillage volume and start-ups and shut-downs of the annual plants in terms of: (a) $\varphi$; (b) $\rho$. 


\section{LONG-TERM OPTIMISATION MODELS FOR HYDRO SCHEDULING SUBJECT TO MINIMUM FLOWS AND MAXIMUM RAMPING RATES}

This chapter is mainly devoted to the achievement of the objectives 1 and 2 of the thesis (as discussed in sec. 1.3) and is developed in three studies (hereinafter referred to as studies 4, 5 and 6 ). The study 4 [Guisández et al. (2015, 2016b,c)] with the latter objective and the studies 5 and 6 with the former one.

The chapter is divided in three parts, one for each study, and each part is structured as in the previous chapter: explanation of the applied methodology, description of the considered case study, and presentation of the main obtained results with their discussion.

\subsection{Influence of the environmental constraints on the water and flow values}

The objectives of the present study, which gives name to this section, correspond, on the one hand, to the objective 2 of the thesis (as discussed in sec. 1.3) and, on the other hand, to a congruent extension of that objective due to the emergence of a new concept: flow value. The presence of this new value arose as a consequence of the consideration of $\mathrm{F}$ in the calculation of the water value. If water value can be defined, from a revenue-based point of view, as the marginal change in the hydropower producer's expected revenue for a marginal change in its hydro (or potential) resources [Reneses et al. (2015)], then, in the same way, flow value is coined in this thesis as the analogous change when varying its "discharge (or cinematic) resources", i.e. its released flow.

In order to obtain an a priori idea of how $\varphi$ and $\rho$ affect water and flow values and with the aim of clarifying the concept of this latter value, a simplified hydro scheduling problem is formulated below in two different forms: one by considering only water value and the other by water and flow values.

- Hydro SCheduling PROBlem CONSIDERING ONLy WATER VAlue

$$
\min \left[-\sum_{t \in T}\left(p_{t} \cdot g_{t}^{v_{t}, f_{t}}\right)-\bar{z}^{v_{t} \max }\right]
$$


subject to:

$$
\begin{aligned}
& v_{t}-v_{t-1}+K_{c 1} \cdot\left(f_{t}-w_{t}\right)=0 ; \forall t \in \mathbb{T} \wedge v_{0}=V_{0} \\
& v_{t}-V^{\max } \leq 0 ; \forall t \in \mathbb{T} \\
& V^{\text {min }}-v_{t} \leq 0 ; \forall t \in \mathbb{T} \\
& f_{t}-F^{\max } \leq 0 ; \forall t \in \mathbb{T} \\
& \varphi_{k}-f_{t} \leq 0 ; \forall t \in \mathbb{T} \\
& f_{t}-f_{t-1}-\rho^{u p} \leq 0 ; \forall t \in \mathbb{T} \wedge f_{0}=F_{0} \\
& -f_{t}+f_{t-1}-\rho^{\text {down }} \leq 0 ; \forall t \in \mathbb{T} \wedge f_{0}=F_{0}
\end{aligned}
$$

Eq.(4.1) is the objective function in which $p_{t}$ represents the energy price during the hour $t$, $g_{t}^{v_{t}, f_{t}}$ refers to the power generated by releasing $f_{t}$ at $v_{t}$ during the hour $t$, and $\bar{z}^{v_{t} \text { max }}$ means the future expected revenue starting from the $\mathrm{V} v_{t^{\max }}$ at the end of the planning period $t^{\max }$. Eq.(4.2) is the state transition equation of $\mathrm{V}$, the so-called water mass balance equation, where $K_{c 1}$ signifies a conversion factor for converting an hourly constant flow expressed in $\mathrm{m}^{3} / \mathrm{s}$ into a volume in $\mathrm{hm}^{3}$. Eqs.(4.3-4.4) are the limits of $\mathrm{V}$ and Eq.(4.5) is the upper one of $q_{t}$. Finally, Eqs.(4.6-4.8) are the environmental constraints.

The resulting Lagrangian function of the above-described problem is:

$$
=\sum_{t \in T}\left\{\begin{array}{r}
\mathcal{L}\left(v_{t}, f_{t}, \lambda_{t}^{v}, \mu_{t}^{V^{\max }}, \mu_{t}^{V^{\min }}, \mu_{t}^{F^{\max }}, \mu_{t}^{\varphi}, \mu_{t}^{\rho^{\text {up }}}, \mu_{t}^{\rho^{\text {down }}}\right) \\
-p_{t} \cdot g_{t}^{v_{t}, f_{t}}+\lambda_{t}^{v} \cdot\left[v_{t}-v_{t-1}+K_{c 1} \cdot\left(f_{t}-w_{t}\right)\right] \\
+\mu_{t}^{V^{\text {max }}} \cdot\left(v_{t}-V^{\text {max }}\right)+\mu_{t}^{V^{\min }} \cdot\left(V^{\text {min }}-v_{t}\right) \\
+\mu_{t}^{F^{\text {max }}} \cdot\left(f_{t}-F^{\max }\right)+\mu_{t}^{\varphi} \cdot\left(\varphi_{k}-f_{t}\right) \\
+\mu_{t}^{\rho^{u p}} \cdot\left(f_{t}-f_{t-1}-\rho^{u p}\right)+\mu_{t}^{\rho^{\text {down }}} \cdot\left(-f_{t}+f_{t-1}-\rho^{\text {down }}\right)
\end{array}\right\}-\bar{z}^{v_{t} \text { max }}
$$


In Eq.(4.9), $\lambda^{\prime} s$ and $\mu^{\prime} s$ are the Lagrange multipliers of the equality and inequality constraints, respectively, which represent the incremental prices of the constraint requirements expressed in units of the objective function [Luenberger (2003)]. Thus, from the first-order KarushKuhn-Tucker conditions [Karush (1939)], it can be obtained:

$$
\begin{gathered}
\frac{\partial \mathcal{L}\left(v_{t}, f_{t}, \lambda_{t}^{v}, \mu_{t}^{V^{\max }}, \mu_{t}^{V^{\min }}, \mu_{t}^{F^{\text {max }}}, \mu_{t}^{\varphi}, \mu_{t}^{\rho^{u p}}, \mu_{t}^{\rho^{d o w n}}\right)}{\partial v_{t}}=0 \\
\Rightarrow-p_{t} \cdot \frac{\partial g_{t}^{v_{t}, f_{t}}}{\partial v_{t}}+\lambda_{t}^{v}-\lambda_{t+1}^{v}+\mu_{t}^{V^{\max }}-\mu_{t}^{V^{\min }}=0 ; \forall t \in \mathbb{T} \mid t<t^{\text {max }} \\
\frac{\partial \mathcal{L}\left(v_{t}, f_{t}, \lambda_{t}^{v}, \mu_{t}^{V^{\max }}, \mu_{t}^{V^{\min }}, \mu_{t}^{F^{\max }}, \mu_{t}^{\varphi}, \mu_{t}^{\rho^{\rho_{p}}}, \mu_{t}^{\rho^{d o w n}}\right)}{\partial v_{t^{\max }}}=0 \\
\Rightarrow-p_{t^{\max }} \cdot \frac{\partial g_{t^{\text {max }}}^{v_{t}, f_{t}}}{\partial v_{t^{\max x}}}+\lambda_{t^{\max }}^{v}+\mu_{t^{\max }}^{V^{\max }}-\mu_{t^{\max }}^{V^{\min }}-\frac{\partial \bar{z}^{v_{t} \max }}{\partial v_{t^{\max }}}=0
\end{gathered}
$$

And from Eq.(4.10-4.11), it is possible to isolate $\lambda_{t}^{v}$ and demonstrate that it represents water value:

$$
\lambda_{t}^{v}=\left\{\begin{array}{l}
p_{t} \cdot \frac{\partial g_{t}^{v_{t}, f_{t}}}{\partial v_{t}}-\mu_{t}^{V^{\max }}+\mu_{t}^{V^{\min }}+\lambda_{t+1}^{v} ; \forall t \in \mathbb{T} \mid t<t^{\max } \\
p_{t^{\max }} \cdot \frac{\partial g_{t^{\max }}^{v_{t}, f_{t}}}{\partial v_{t^{\max x}}}-\mu_{t^{\max }}^{V^{\max }}+\mu_{t^{\max }}^{V^{\min }}+\frac{\partial \bar{z}_{t^{\max }}}{\partial v_{t^{\max }}} ; t=t^{\max }
\end{array}\right.
$$

Applying the first-order Karush-Kuhn-Tucker conditions, it can be also obtained:

$$
\begin{array}{r}
\frac{\partial \mathcal{L}\left(v_{t}, f_{t}, \lambda_{t}^{v}, \mu_{t}^{V^{\text {max }}}, \mu_{t}^{V^{\text {min }}}, \mu_{t}^{F^{\text {max }}}, \mu_{t}^{\varphi}, \mu_{t}^{\rho^{u p}}, \mu_{t}^{\rho^{\text {down }}}\right)}{\partial f_{t}}=0 \\
\Rightarrow-p_{t} \cdot \frac{\partial g_{t}^{v_{t}, f_{t}}}{\partial f_{t}}+K_{c 1} \cdot \lambda_{t}^{v}+\mu_{t}^{F^{\text {max }}}-\mu_{t}^{\varphi}-\mu_{t+1}^{\rho^{u p}}+\mu_{t}^{\rho^{u p}}+\mu_{t+1}^{\rho^{d o w n}}-\mu_{t}^{\rho^{d o w n}}=0 \\
\forall t \in \mathbb{T} \mid t<t^{\text {max }}
\end{array}
$$

According to those conditions, the multipliers $\mu^{\prime} s$ are non-negative and only zero if their respective constraints are not active [Luenberger (2003)]. Then, for a problem with only $\varphi$, Eq.(4.13) can be re-written as follows:

$$
\lambda_{t}^{v}=\frac{p_{t} \cdot \frac{\partial g_{t}^{v_{t}, f_{t}}}{\partial f_{t}}-\mu_{t}^{F^{\max }}+\mu_{t}^{\varphi}}{K_{c 1}} ; \rho=0 \wedge \forall t \in \mathbb{T} \mid t<t^{\text {max }}
$$

Because of Eqs.(4.5-4.6) cannot be active at the same time, $\mu_{t}^{F^{\max }}$ and $\mu_{t}^{\varphi}$ cannot be nonzero at the same moment since those are their respective constraints. Eq.(4.14) demonstrates that water value is related with $\varphi$ through the corresponding multiplier $\mu_{t}^{\varphi}$. Considering two problems with different $\varphi$ but the same input data, generation characteristic and constraints, Eq.(4.14) would indicate that, with a linear generation characteristic, as $\varphi$ increases, water 
value would also increase, assuming that, in general, $\mu_{t}^{\varphi}$ grows as $\varphi$ increases. In a case with a non-linear generation characteristic, the influence of $\varphi$ on water value depends also on the first derivative of the generation characteristic with respect to $f_{t}$.

For a problem with only $\rho$, and considering that $f_{t}$ is not at its limits, Eq.(4.13) can be re-written as follows:

$$
\begin{array}{r}
\lambda_{t}^{v}=\frac{p_{t} \cdot \frac{\partial g_{t}^{v_{t}, f_{t}}}{\partial f_{t}}+\left(\mu_{t+1}^{\rho^{u p}}-\mu_{t}^{\rho^{u p}}\right)-\left(\mu_{t+1}^{\rho^{d o w n}}-\mu_{t}^{\rho^{d o w n}}\right)}{K_{c 1}} ; \\
\varphi=0 \wedge 0<f_{t}<F^{\max } \wedge \forall t \in \mathbb{T} \mid t<t^{\max }
\end{array}
$$

Eq.(4.15) indicates that water value is related with $\rho^{u p}$ and $\rho^{\text {down }}$ through the corresponding multipliers. Considering two problems with different $\rho$ but the same input data, generation characteristic and constraints, Eq.(4.15) would indicate that, with a linear generation characteristic, water value would increase if $\left(\mu_{t+1}^{\rho^{u p}}-\mu_{t}^{\rho^{u p}}\right)$ increases or $\left(\mu_{t+1}^{\rho^{\text {down }}}-\mu_{t}^{\rho^{\text {down }}}\right)$ decreases. In order to anticipate the variation in $\left(\mu_{t+1}^{\rho^{u p}}-\mu_{t}^{\rho^{u p}}\right)$ or $\left(\mu_{t+1}^{\rho^{\text {down }}}-\mu_{t}^{\rho^{\text {down }}}\right)$ between these latter problems, it can be analysed Fig. 4.1, where the hypothetical resulting water release $f_{t}$ of a set of problems with different $\rho$ is depicted. From this figure, it can be deduced that $\left(\mu_{t+1}^{\rho^{u p}}-\mu_{t}^{\rho^{u p}}\right)$ increases and $\left(\mu_{t+1}^{\rho^{\text {down }}}-\mu_{t}^{\rho^{\text {down }}}\right)$ decreases as the severity of $\rho$ decreases, and therefore water value would grow. In a case with a non-linear generation characteristic, the influence of $\rho$ on water value depends also on the first derivative of the generation characteristic with respect to $f_{t}$.

Despite the foregoing, the conclusions of this analysis should be confirmed with some numerical results.

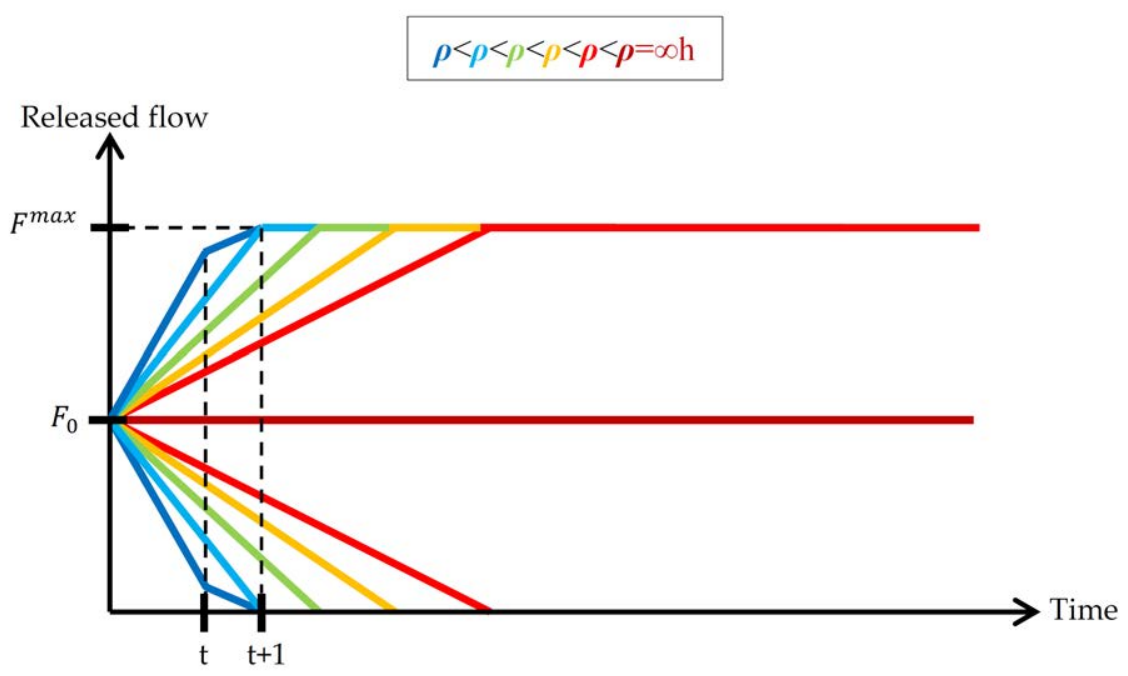

Figure 4.1.: Examples of different $\rho$.

- Hydro scheduling PRoblem CONSidering WATER ANd flow VAlues

$$
\min \left[-\sum_{t \in T}\left(p_{t} \cdot g_{t}^{v_{t}, f_{t}}\right)-\bar{z}^{v_{t} \max , f_{t} \max }\right]
$$


subject to: Eqs.(4.2-4.6) and

$$
\begin{aligned}
& f_{t}-f_{t-1}-d_{t}=0 ; \forall t \in \mathbb{T} \mid f_{0}=F_{0} \\
& d_{t}-\rho^{u p} \leq 0 ; \forall t \in \mathbb{T} \\
& -d_{t}-\rho^{\text {down }} \leq 0 ; \forall t \in \mathbb{T}
\end{aligned}
$$

Eq.(4.16) is the objective function in which $\bar{z}^{v_{t} \max , f_{t} \max }$ means the future expected revenue starting from the $\mathrm{V} v_{t^{\max }}$ and the $\mathrm{F} f_{t^{\max }}$ (released through the hydro units, bottom outlets and spillways) at the end of the planning period $t^{\max }$. Eq.(4.17) is the analogous equation of Eq.(4.2) for F, which may be named operational inertia equation until a better term can be found, where $d_{t}$ indicates the decision which corresponds to the hourly variation of F. Finally, Eqs.(4.18-4.19) are the $\rho$ constraints.

The resulting Lagrangian function of the current problem is:

$$
=\sum_{t \in T}\left\{\begin{array}{r}
\mathcal{L}\left(d_{t}, v_{t}, f_{t}, \lambda_{t}^{v}, \lambda_{t}^{f}, \mu_{t}^{V^{\text {max }}}, \mu_{t}^{V^{\text {min }}}, \mu_{t}^{F^{\text {max }}}, \mu_{t}^{\varphi}, \mu_{t}^{\rho^{\text {up }}}, \mu_{t}^{\rho^{\text {down }}}\right) \\
-p_{t} \cdot g_{t}^{v_{t}, f_{t}}+\lambda_{t}^{v} \cdot\left[v_{t}-v_{t-1}+K_{c 1} \cdot\left(f_{t}-w_{t}\right)\right]+\lambda_{t}^{f} \cdot\left(f_{t}-f_{t-1}-d_{t}\right) \\
+\mu_{t}^{V^{\max }} \cdot\left(v_{t}-V^{\max }\right)+\mu_{t}^{V^{\min }} \cdot\left(V^{\text {min }}-v_{t}\right) \\
+\mu_{t}^{F^{\max }} \cdot\left(f_{t}-F^{\max }\right)+\mu_{t}^{\varphi} \cdot\left(\varphi_{k}-f_{t}\right) \\
+\mu_{t}^{\rho^{u p}} \cdot\left(d_{t}-\rho^{u p}\right)+\mu_{t}^{\rho^{\text {down }}} \cdot\left(-d_{t}-\rho^{\text {down }}\right) \\
-\bar{z}^{v_{t} \max , f_{t} \text { max }}
\end{array}\right.
$$

As before, $\lambda^{\prime} s$ and $\mu^{\prime} s$ are the Lagrange multipliers of the equality and inequality constraints in Eq.(4.20), respectively.

From the first-order Karush-Kuhn-Tucker conditions [Karush (1939)] it can be obtained:

$$
\begin{aligned}
& \frac{\partial \mathcal{L}\left(d_{t}, v_{t}, f_{t}, \lambda_{t}^{v}, \lambda_{t}^{f}, \mu_{t}^{V^{\max }}, \mu_{t}^{V^{\text {min }}}, \mu_{t}^{F^{\text {max }}}, \mu_{t}^{\varphi}, \mu_{t}^{\rho^{\text {up }}}, \mu_{t}^{\rho^{\text {down }}}\right)}{\partial f_{t}}=0 \\
& \Rightarrow-p_{t} \cdot \frac{\partial g_{t}^{v_{t}, f_{t}}}{\partial f_{t}}+K_{c 1} \cdot \lambda_{t}^{v}+\lambda_{t}^{f}-\lambda_{t+1}^{f}+\mu_{t}^{F^{\max }}-\mu_{t}^{\varphi}=0 \\
& \forall t \in \mathbb{T} \mid t<t^{\max }
\end{aligned}
$$




$$
\begin{aligned}
& \frac{\partial \mathcal{L}\left(d_{t}, v_{t}, f_{t}, \lambda_{t}^{v}, \lambda_{t}^{f}, \mu_{t}^{V^{\max }}, \mu_{t}^{V^{\min }}, \mu_{t}^{F^{\max }}, \mu_{t}^{\varphi}, \mu_{t}^{\rho^{u p}}, \mu_{t}^{\rho^{\text {down }}}\right)}{\partial f_{t^{\max }}}=0 \\
& \Rightarrow-p_{t^{\max }} \cdot \frac{\partial g_{t^{\max }}^{v_{t}, f_{t}}}{\partial f_{t^{\max }}^{\max }}+K_{c 1} \cdot \lambda_{t^{\max }}^{v}+\lambda_{t^{\max }}^{f}+\mu_{t^{\max }}^{F^{\max }}-\mu_{t^{\max }}^{\varphi}-\frac{\partial \bar{z}^{v_{t} \max }, f_{t^{\max }}}{\partial f_{t^{\max }}}=0 ; \\
& \forall k \in \mathbb{K}
\end{aligned}
$$

And from Eq.(4.21-4.22), it is possible to isolate $\lambda_{t}^{f}$ and demonstrate that it represents flow value:

$$
\lambda_{t}^{f}=\left\{\begin{array}{c}
p_{t} \cdot \frac{\partial g_{t}^{v_{t}, f_{t}}}{\partial f_{t}}-K_{c 1} \cdot \lambda_{t}^{v}-\mu_{t}^{F^{\text {max }}}+\mu_{t}^{\varphi}+\lambda_{t+1}^{f} ; \forall t \in \mathbb{T} \mid t<t^{\text {max }} \\
p_{t^{\max }} \cdot \frac{\partial g_{t^{\text {max }}}^{v_{t}, f_{t}}}{\partial v_{t^{\max x}}}-K_{c 1} \cdot \lambda_{t^{\max }}^{v}-\mu_{t^{\max }}^{F^{\max }}+\mu_{t^{\max }}^{\varphi}+\frac{\partial \bar{z}^{v_{t} \max , f_{t} \max }}{\partial v_{t^{\max }}} ; t=t^{\text {max }}
\end{array}\right.
$$

Eq.(4.23) shows, logically, that flow value is function of $\mathrm{F}$ and, besides, reveals that flow and water values are related with each other, and that therefore, the former also varies as a function of $\mathrm{V}$ and the time of the year (as discussed in sec.1.2).

Finally, applying the first-order Karush-Kuhn-Tucker conditions, it can be also obtained:

$$
\begin{array}{r}
\frac{\partial \mathcal{L}\left(d_{t}, v_{t}, f_{t}, \lambda_{t}^{v}, \lambda_{t}^{f}, \mu_{t}^{V^{\max }}, \mu_{t}^{V^{\min }}, \mu_{t}^{F^{\max }}, \mu_{t}^{\varphi}, \mu_{t}^{\rho^{u p}}, \mu_{t}^{\rho^{d o w n}}\right)}{\partial d_{t}}=0 \\
\Rightarrow-\lambda_{t}^{f}+\mu_{t}^{\rho^{u p}}-\mu_{t}^{\rho^{d o w n}}=0 ; \forall t \in \mathbb{T}
\end{array}
$$

As it can be seen, Eq.(4.24) indicates that flow value can be positive or negative, depending on what $\rho$ is activated.

\subsubsection{Methodology}

Two annual models (modVstu4 and modVFstu4), based on a hybrid combination of stochastic discrete DP and deterministic mixed LP, were developed in this study. The methodology applied in this study moved from the deterministic approach (used in the previous studies) to the stochastic one since the latter is more widely used to determine the water value and, in general, to make any long- or mid-term decision to be used in short-term hydro scheduling (as discussed in sec. 1.2). As in the previous studies, the combined optimisation-simulation approach (as discussed in sec. 2.1.1) was also applied by recalculating, with the real generation characteristic of the plant, the power outputs and the resulting revenue from the decisions obtained in all LP optimisations.

Water inflow and energy price were considered as state variables in the DP algorithms and as input variables in the LP ones. The stochasticity of these variables in the DP algorithms was modelled by Markov chains whose orders were selected through the Akaike information criterion, whose numbers of classes were determined using the discretisation thickening 
method, and whose extreme classes represented the historical weekly extreme values of the variables (as discussed in sec. 2.3.1). Both variables in the LP algorithms were disaggregated into hourly values from the weekly ones used in the Markov chains by weighting the historical average weekly profile of each variable in every week by a ratio between the considered value in its respective chain and the mean value of the said average profile (see an example in Fig. 4.2). Furthermore, modVstu 4 and modVFstu 4 also use weekly evaporation rate as input variable by evaluating its average historical values in only one scenario.

(a)

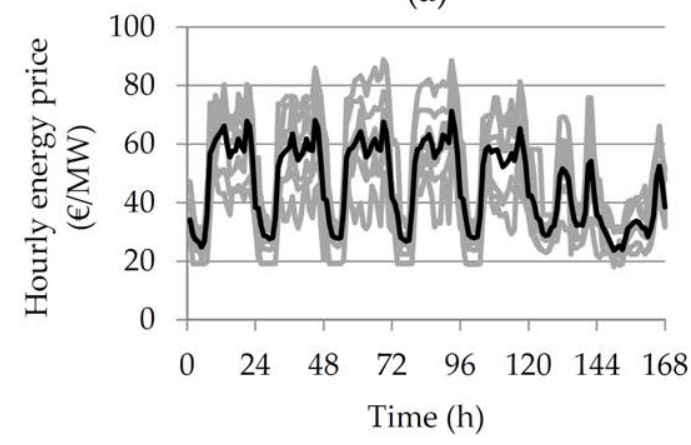

(c)

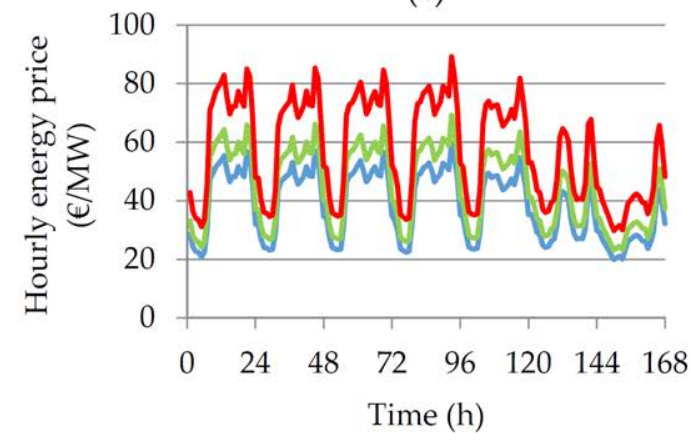

(b)

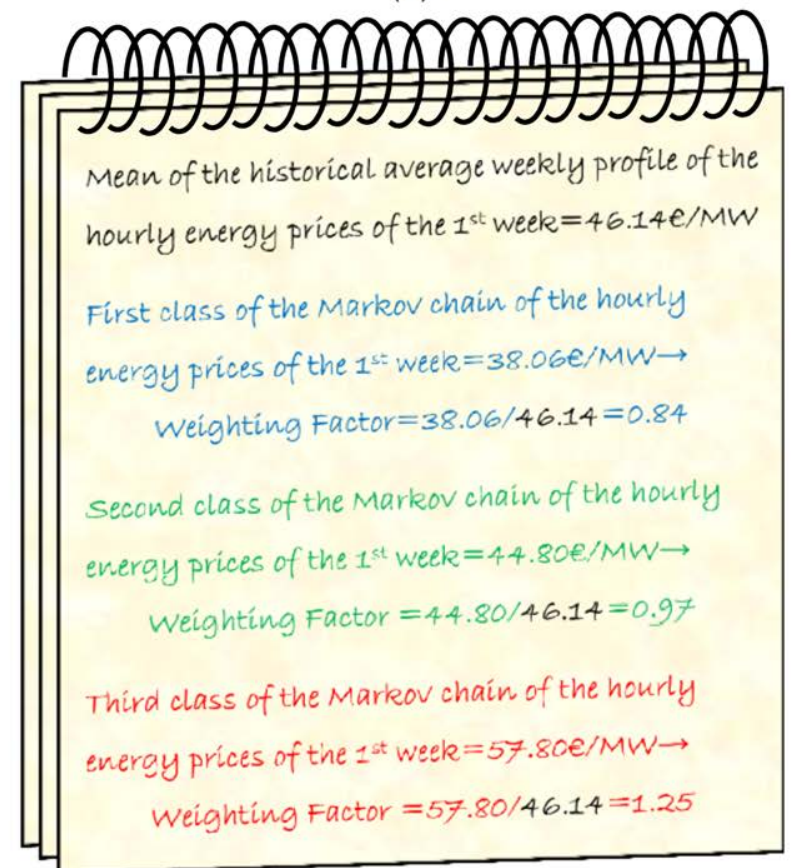

Figure 4.2.: (a) Historical weekly profiles (grey lines) and their average (black line) of the hourly energy prices of the first week of the water year; (b) Calculation of the weighting factors for disaggregation of the classes of the Markov chain of the energy price of the first week of the water year; (c) Hourly disaggregations of the three classes of the Markov chain of the energy price of the first week of the water year.

Stabilisation of the expected revenue increment (as discussed in sec. 2.2.2) was selected as the convergence criterion of the stochastic discrete DP for its simplicity. In comparison with the study 3 (as discussed in sec.3.3.1), the selected value of this convergence criterion was increased and $\mathrm{V}$ was discretised in less states in order to ease the convergence and to reduce the computational burden, respectively.

\section{- Description of modVstu4}

The purpose of modVstu4, Eqs. (A.2, A.6, A.14, A.19, A.22, A.25-A.26, A.28-A.29, A.32, A.47-A.48, A.50-A.51, A.53, A.61-A.71 and A.73-A.84) is to calculate the water value. This hybrid optimisation model is an improved version of mod2 Vstu2 (as discussed in sec. 3.2.1) but its process is different. These are the steps of the model in order: 
1. The DP module decomposes the considered annual problem into weekly subproblems.

2. Starting from the last week of the year, the LP module optimises the decisions of all subproblems contained in the considered week.

3. The DP module recalculates, with the real generation characteristic of the plant, the power outputs according to the decisions provided by the LP module of each subproblem and obtains the resulting revenue.

4. The DP module calculates the water value at the beginning of the considered week.

5. Second, third and fourth steps are repeated backwards until the beginning of the year for the rest of the weeks using the last-obtained water value.

6. The DP module checks if the relative interannual variations of the expected revenue at the beginning of each week converge. If the answer is yes, the process stops; otherwise the process is repeated from the second step but now considering the last-obtained water value at the end of the last week.

The DP module, Eqs. (A.2, A.6 and A.14), is aimed at maximising the hydropower plant revenue in the day-ahead electricity market throughout a planning period of one year divided into weekly time steps. It uses the volume released from the reservoir throughout each week as decision variable and, as state variables, $\mathrm{V}$ at the beginning of each week (discretised in nine equidistant values from the dead volume to the maximum one; as discussed in sec. 2.2.2), the current weekly water inflow, and the current average weekly energy price.

The LP module, Eqs. (A.19, A.22, A.25-A.26, A.28-A.29, A.32, A.47-A.48, A.50-A.51, A.53, A.61-A.71 and A.73-A.84) is aimed at maximising the hydropower plant revenue in the day-ahead electricity market throughout a planning period of one week, divided into hourly time steps, plus the future expected revenue, given by the water value. It uses $\mathrm{F}$ during each hour as decision variable and hourly water inflow and energy price as input variables.

The objective function of the LP module (Eq. A.19) is subject to the following constraints: $\varphi$ (Eq. A.22); up and down $\rho$, Eqs. (A.25-A.26); water mass balance (Eq. A.28); evaporation losses (Eq. A.29); initial V (Eq. A.32); maximum legal storage capacity of the reservoir (Eq. A.47); maximum technical storage capacity of the reservoir (Eq. A.48); dead storage capacity (Eq. A.50); flow released through the hydro units (Eq. A.51); plant generation characteristic model of one non-concave curve, Eqs. (A.53 and A.61-A.62); inter-hourly variation of the generated power (Eq. A.63); start-up and shut-down of the hydro units, Eqs. (A.64-A.68); maximum technical plant flow, Eqs. (A.69-A.71); maximum flow through the bottom outlets (Eq. A.73); maximum flow over the spillways, Eqs. (A.74-A.78); inter-hourly variation of the total flow released through the bottom outlets and/or over the spillways (Eq. A.79) and the water value at the end of each subproblem, Eqs. (A.80-A.84). 


\section{- Description of modVFstu4}

The purpose of modVFstu4, Eqs. (A.4, A.6, A.8, A.15, A.20, A.22, A.25-A.26, A.28-A.29, A.32, A.40-A.41, A.47-A.48, A.50-A.51, A.53, A.61-A.71, A.73-A.79 and A.85-A.95) is to calculate the water and flow values. The structure of modVFstu4 is practically identical to modVstu4 and its main variations from that model are the use of $\mathrm{F}$ as state variable (Eq. A.8), with the same discretisation used in mod2stu3 (as discussed in sec.3.3.1), and the consideration of the water value together with the flow value at the end of each subproblem, Eqs. (A.20 and A.85-A.95), modelled through the one-dimensional method (as discussed in sec. 2.4.1).

\subsubsection{Case study}

Plant I (Tab. B.2), the same peak hydropower plant as in the studies 1 and 2, located in the northwest of Spain (Fig. B.1), was the selected one for carrying out the current study. In this case, its modelling was improved by the introduction of the hydraulic losses in its performance curves (see in Fig. B.6) as well as by the consideration of the maximum available flows of its bottom outlets and spillways according to the current V (see in Fig. B.4). The head-storage curve of the plant was also used (see in Fig. B.18(a)).

Once more, the daily water inflows and the hourly energy prices were taken from the web page of the Centre for Public Works Studies and Experimentation [CEDEX (2013)] and from the web page of the Iberian Electricity Market Operator [OMIE (2011)], respectively. The weekly evaporation rates were estimated by application of the empirical temperature-based formula proposed in Dragoni and Valigi (1994) and of the average monthly temperatures taken from the web page of Spanish Statistical Office [INE (1960)]. The analysed scenarios of the input variables as well as the evaluated magnitudes of $\varphi$ and $\rho$, expressed in relative terms following the methodology of the study 1 (as discussed in sec. 3.1.1), are noted in Tab.4.1.

The above-described optimisation models (sec. 4.1.1) were implemented in MATLAB ${ }^{\circledR}$, each subproblem being solved using CPLEX ${ }^{\mathrm{TM}}$ under $\mathrm{GAMS}^{\mathrm{TM}}$, on three computers, totaling 32 threads (used each pair in parallel [Baslis and Bakirtzis (2011)] at each computer): two Intel ${ }^{\circledR}$ Core $^{\mathrm{TM}}$ i7 at $2.4 \mathrm{GHz}$ and $8 \mathrm{~GB}$ RAM and one Intel ${ }^{\circledR} \mathrm{Xeon}^{\circledR} \mathrm{E} 5$ at $3.1 \mathrm{GHz}$ and $64 \mathrm{~GB}$ RAM. Following again the suggestion in Carvalho and Pinto (2006), a small sensitivity analysis of the performance of the LP algorithms of the models was done, using as a basis the results of those carried out in the studies 2 and 3 (as discussed in sec. 3.2.2 and sec. 3.3.2). The selected parameters are given in Tab. 4.2.

A total of 36 estimations of water and flow values were undertaken in this study; 28 considering only $\mathrm{V}$ and 8 considering $\mathrm{V}$ and $\mathrm{F}$. These estimations were decomposed into 4,841,928 short-term subproblems: 1,107,288 were solved by modVstu4 and 3,734,640 by modVFstu4. 
Table 4.1.: Input variable scenarios and environmental constraints used in the study 4 .

\begin{tabular}{|c|c|c|}
\hline CONCEPT & SCENARIOS & DESCRIPTION \\
\hline Water inflows & 5 Markov classes of lag 1 & Hourly \\
\hline Energy prices & 3 Markov classes of lag 1 & Hourly \\
\hline Evaporation rates & Average & Weekly \\
\hline \multirow[t]{2}{*}{$(\varphi, \rho)$} & $\begin{array}{c}(0 \%, 0 \mathrm{~h}) ;(0.5 \%, 0 \mathrm{~h}) ;(1 \%, 0 \mathrm{~h}) ; \\
(2 \%, 0 \mathrm{~h}) ;(3 \%, 0 \mathrm{~h}) ;(4 \%, 0 \mathrm{~h}) ; \\
(5 \%, 0 \mathrm{~h}) ;(8 \%, 0 \mathrm{~h}) ;(0 \%, 6 \mathrm{~h}) ; \\
(0 \%, 12 \mathrm{~h}) ;(0 \%, 24 \mathrm{~h}) ;(0 \%, 36 \mathrm{~h}) ; \\
(0 \%, 48 \mathrm{~h}) ;(0 \%, 60 \mathrm{~h}) ;(0 \%, 72 \mathrm{~h}) ; \\
(5 \%, 60 \mathrm{~h})^{a}\end{array}$ & $\begin{array}{l}\varphi_{\text {Oct.-Dec. }}=0.75 \varphi ; \\
\varphi_{\text {Jan.-Mar. }}=1.75 \varphi ; \\
\varphi_{\text {Apr.-Jun. }}=1.20 \varphi ; \\
\varphi_{\text {Jul.-Sep. }}=0.30 \varphi ; \\
\rho_{1-k_{\text {max }}}^{\text {up }}=0.75 \rho ; \\
\rho_{1-k_{\max }^{\text {down }}}^{\text {don }}=1.50 \rho\end{array}$ \\
\hline & $\begin{array}{c}(0.5 \%, 0 \mathrm{~h}) ;(1 \%, 0 \mathrm{~h}) \\
(2 \%, 0 \mathrm{~h}) ;(3 \%, 0 \mathrm{~h}) \\
(4 \%, 0 \mathrm{~h}) ;(5 \%, 0 \mathrm{~h}) \\
(8 \%, 0 \mathrm{~h})\end{array}$ & $\begin{array}{c}\varphi_{\text {Oct.-Dec. }}=\varphi \\
\varphi_{\text {Jan.-Mar. }}=\varphi \\
\varphi_{\text {Apr.-Jun. }}=\varphi \\
\varphi_{\text {Jul.-Sep. }}=\varphi\end{array}$ \\
\hline
\end{tabular}

${ }^{a}$ Planned to be imposed by the water basin authority.

Table 4.2.: Characteristic parameters of the LP algorithms of the study 4 .

\begin{tabular}{|c|c|}
\hline PARAMETER & VALUE \\
\hline \hline Relative optimality criterion (\%) & 1 \\
\hline Time limit for each subproblem (s) & 10 \\
\hline
\end{tabular}

\subsubsection{Main results and discussion}

The averages across $\mathrm{V}$ and the seasons of the water value in relative terms with respect to the scenario without environmental constraints are included in Fig. 4.3 for every considered $\varphi$ and $\rho$. This figure corroborates the conclusions of the discussion carried out in sec.4.1, i.e. the water value increased (almost linearly) with $\varphi$ and decreased (quadratically) with $\rho$. These opposing effects are also caused by the head-storage relationship. Suppose two separate problems aimed at maximising the future revenue of a peak hydropower plant starting from two different $\mathrm{V}$. Regardless of the future values of the input variables, the policy followed by the plant from the upper $\mathrm{V}$ would have a bigger average head than the one corresponding to the lower $\mathrm{V}$, and therefore the impact of $\varphi$ would be higher for the lower $\mathrm{V}$ than for the upper one since the detraction of water represents a larger reduction of head for the lower $\mathrm{V}$ than for the upper one. As regards $\rho$, its impact would be bigger for the upper $\mathrm{V}$ than for the lower one because the average head is larger at the upper $\mathrm{V}$ than at the lower one. In both constraints, the greater their values, the greater their impacts on the water value. 


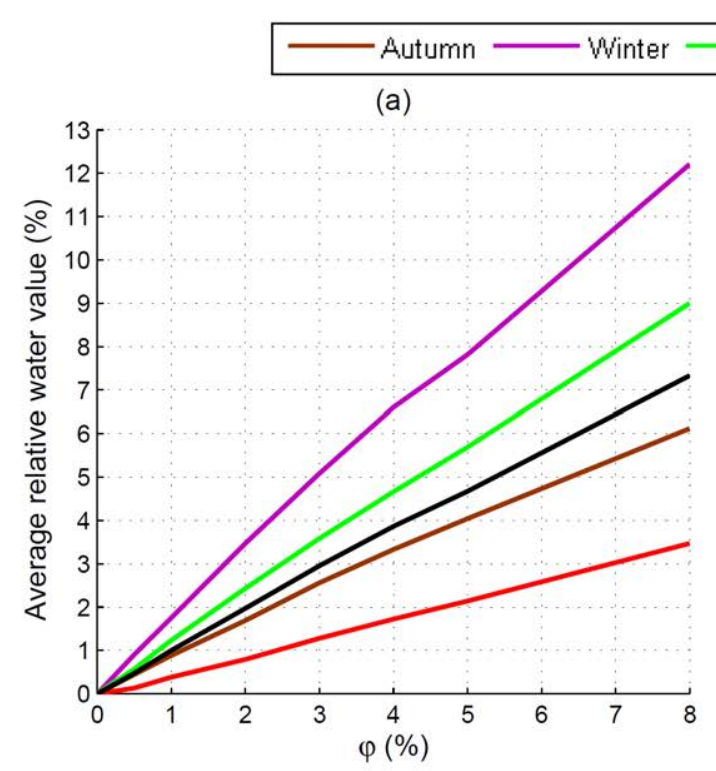

Spring - Summer - Annual

(b)

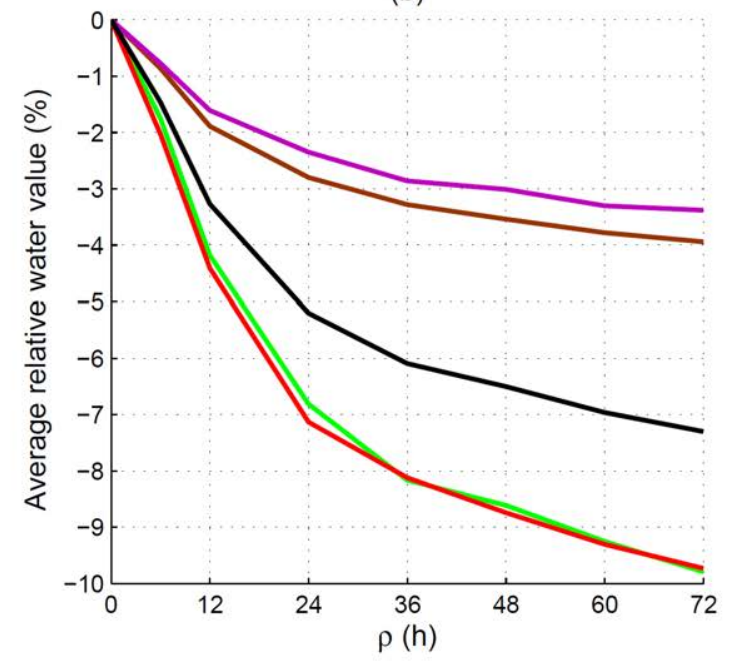

Figure 4.3.: Average relative water values of the plant I in terms of: (a) $\varphi$; (b) p.

Returning to Fig. 4.3, it can also be observed that the effect of $\varphi$ was more significant in spring and winter, whereas $\rho$ had more influence in spring and summer. It is must be noted that Fig. 4.3 shows relative values but the water value in absolute terms was higher in spring and summer than in autumn and winter as it can be seen in Fig. 4.4. On the other hand, it is important to bear in mind that $\varphi$ had its largest value during spring and winter (see in Tab. 4.1). Even though $\varphi$ usually varies seasonally, Fig. 4.5(a)-(b) show, for the sake of clarity, the same results as Fig. 4.3(a) and Fig. 4.4(a) but considering $\varphi$ constant along the year. In addition, Fig. 4.5(c) shows the impact of $\varphi$ on the water value in $k € / \mathrm{hm} 3$. As regards $\rho$, its higher impact on the water value in spring and summer is to a certain extent expected, since the lower the water inflow, the higher the economic value of the the operational flexibility.

- Autumn — Winter - Spring - Summer - Annual
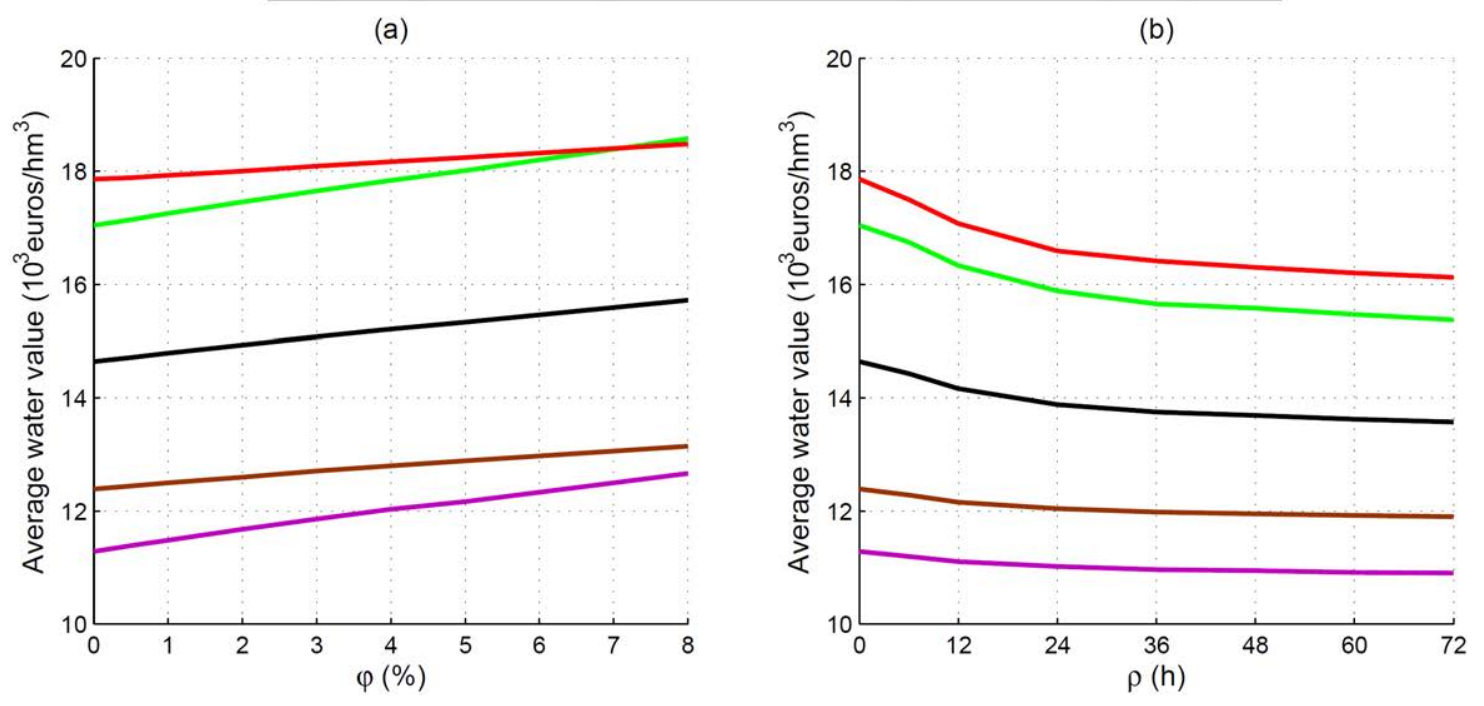

Figure 4.4.: Average water values of the plant I in terms of: (a) $\varphi$; (b) $\rho$. 


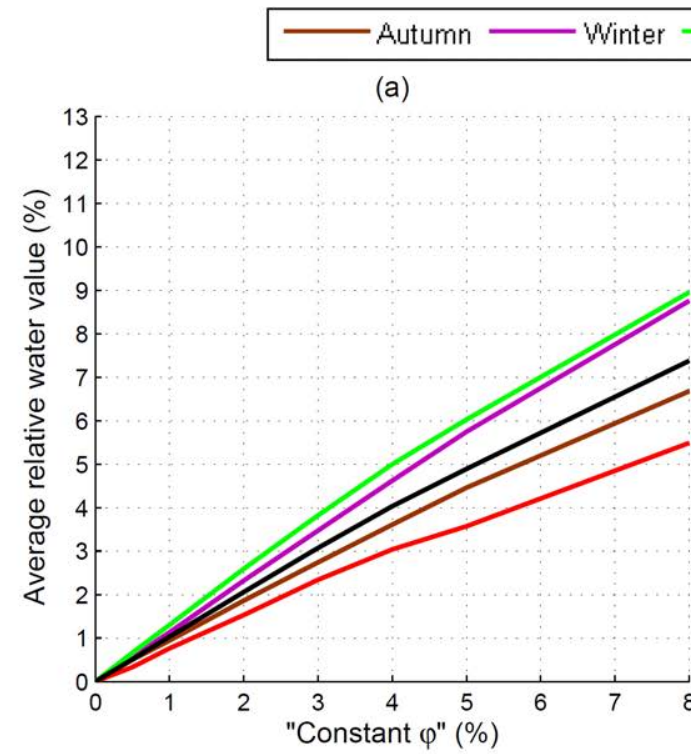

Spring - Summer - Annual

(b)

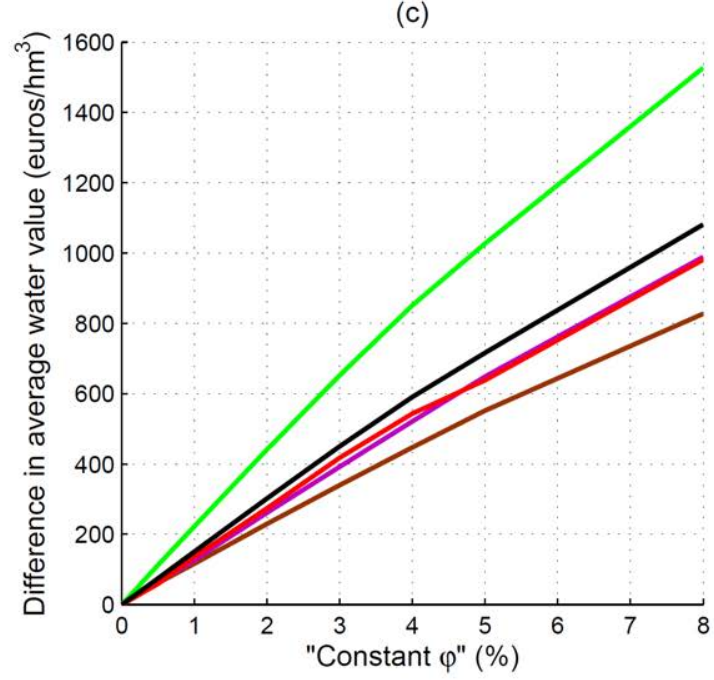

Figure 4.5.: (a) Average relative water values of the plant I in terms of constant $\varphi$; (b) Average water values of the plant I in terms of constant $\varphi$; (c) Difference in average water values of the plant $I$ in terms of constant $\varphi$.

Regarding the flow values, their absolute averages across $\mathrm{F}$ and the seasons are showed in Fig. 4.6 for every evaluated p. It is worth noting that unlike the water value, which can be below zero only under very specific conditions (such as considering negative energy prices or a spillage penalty), the flow value is able to reach negative magnitudes (see Eq.(4.24)) under normal conditions in order to show the expected profitability of finishing the weekly operation at low $\mathrm{F}$ (this is the reason why they are not represented in relative terms with respect to any scenario). Fig. 4.6 seems to suggest that the flow value tends to increase with $\rho$ in the wettest season and conversely in the driest one. This effect is coherent with the seasonal nature of the water value, which is usually decreasing with the amount of water inflow (as discussed in sec. 1.2), since the more valuable is the water stored into the reservoir, the less economically interesting is scheduling an aggressive profile at the beginning of the week and vice versa. 


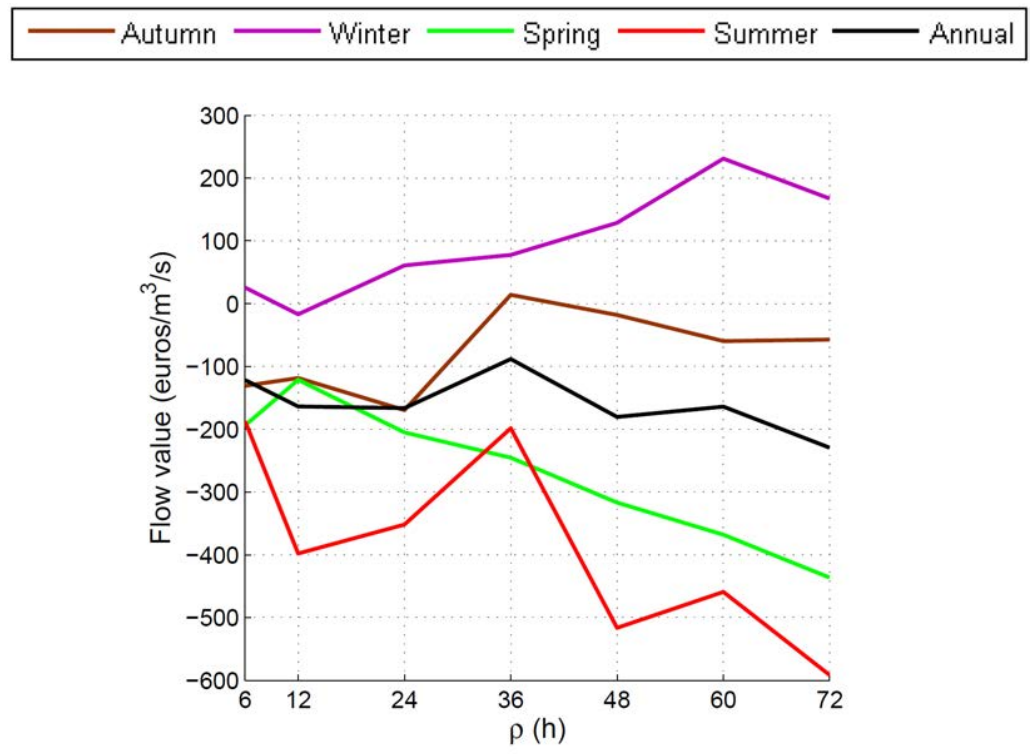

Figure 4.6.: Average flow values of the plant I in terms of $\rho$.

In Fig. 4.7, the level curves of the water value according to $\mathrm{V}$ across the year for the scenario without environmental constraints are represented. As it can be observed, the evolution of the water value was consistent with Fig. B.20(a) and Tab. B.2: on the one hand, the lowest average absolute values appeared in winter and the highest ones in summer, and, on the other hand, the lowest relative values of each week were given at the maximum $\mathrm{V}$ in winter whereas those during summer were located in the minimum $\mathrm{V}$. both phenomena are presented in all scenarios (see in Fig. 4.8).

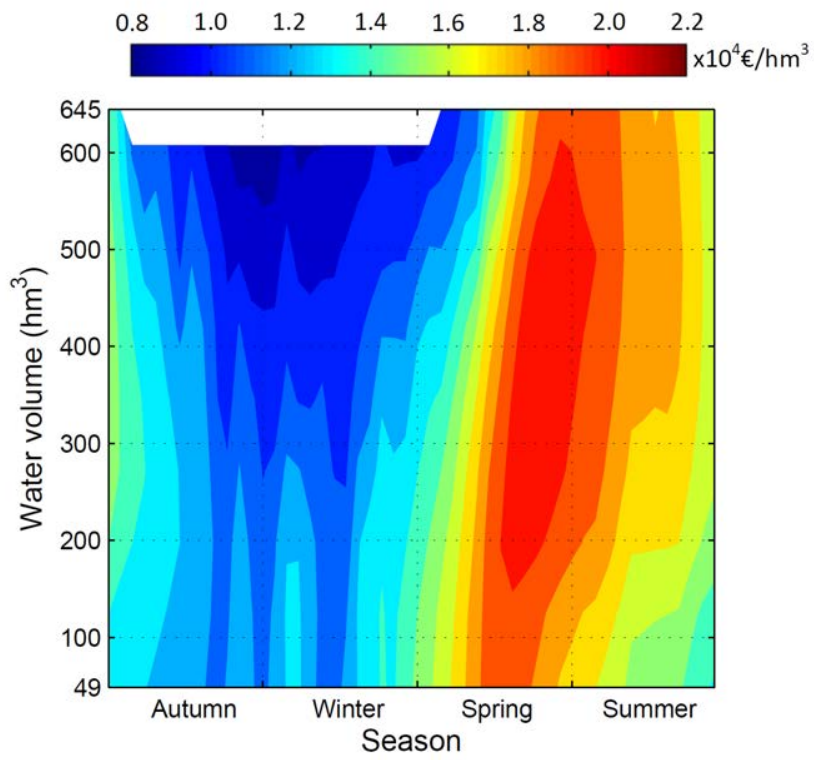

Figure 4.7.: Water value level curves of the plant I of the scenario without environmental constraints. 
(a)

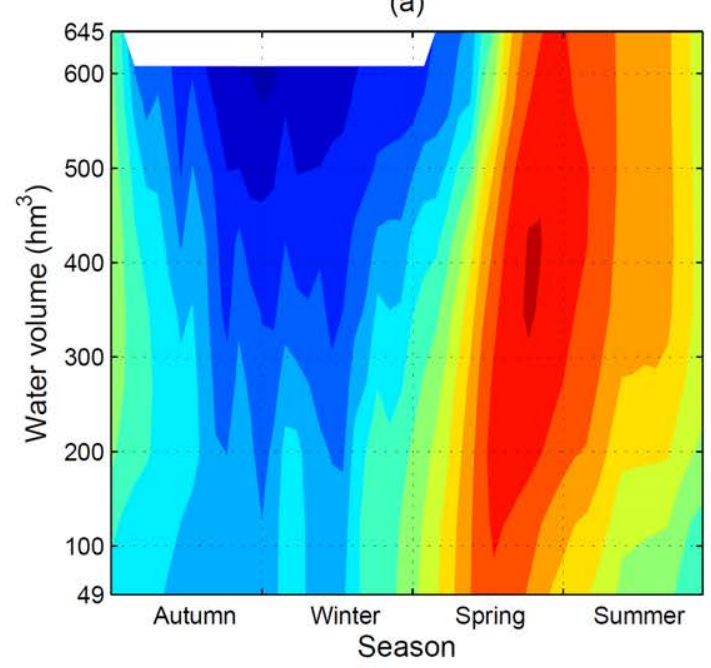

(c)

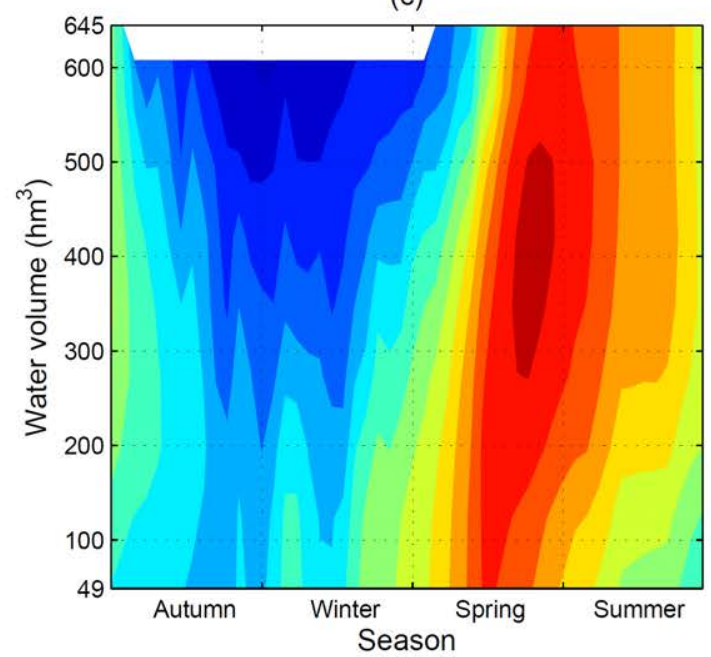

(e)

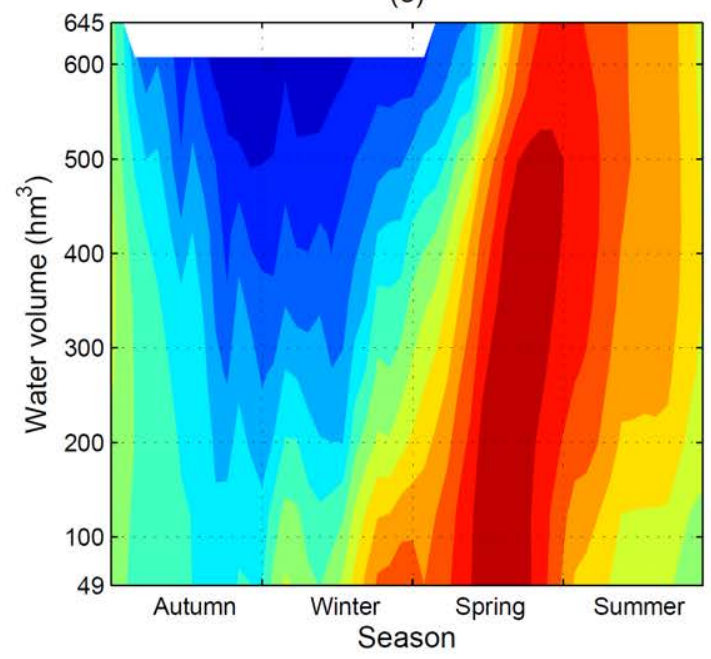

(b)

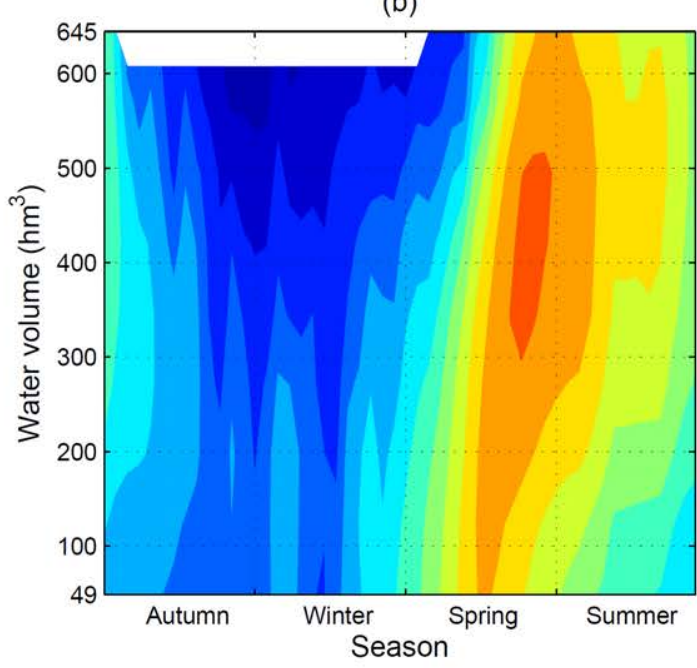

(d)

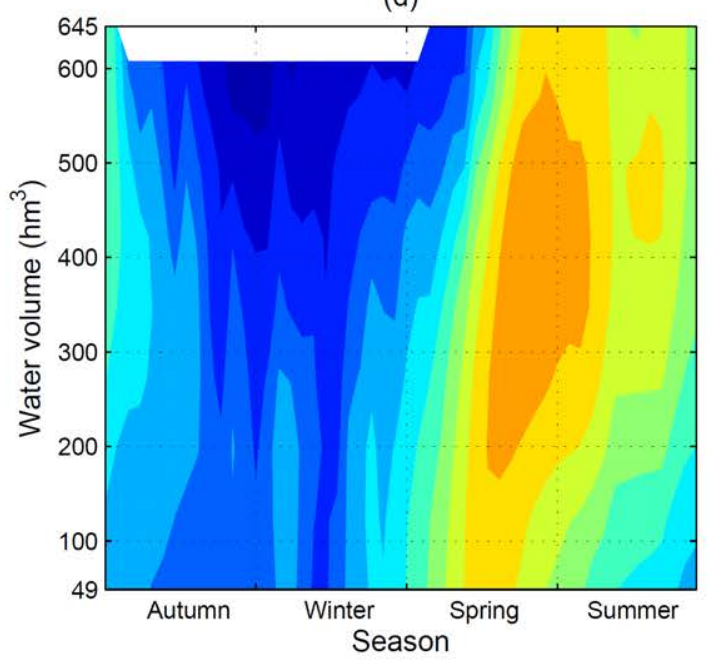

(f)

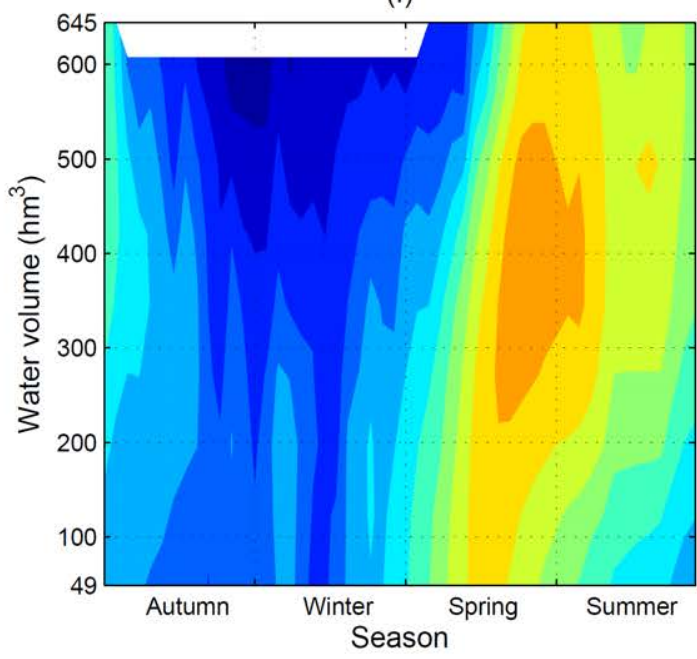

Figure 4.8.: Water value level curves of the plant I with: (a) $\varphi=2 \%$; (b) $\rho=24$ h; (c) $\varphi=4 \%$; (d) $\rho=48 \mathrm{~h}$; (e) $\varphi=8 \%$; (f) $\rho=72 \mathrm{~h}$. 
The former of the just-mentioned phenomenon is a well-known consequence of the increasing risk of spill with $\mathrm{V}$ and with the amount of water inflow (as discussed in sec. 1.2). The latter phenomenon, which had not been reported in the literature yet until Guisández et al. (2016b), occurs not only because of the lack of risk of spill during summer, but also the subsequent prominence of the plant generation characteristic.

Suppose another theoretical problem aimed at maximising the future revenue of a peak hydropower plant starting from three different $\mathrm{V}$, assuming that future expected water inflows are null. In this extremely dry scenario, it is obvious that the difference in revenue between the lower $\mathrm{V}$ and the intermediate $\mathrm{V}$, and between the intermediate $\mathrm{V}$ and the upper $\mathrm{V}$, is due to both the plant generation characteristic and the difference in the initial $\mathrm{V}$, whereas the difference in water value depends only on the former.

With the aim of supporting this idea, the water value was calculated in five additional experiments, each with a constant weekly profile of both hourly water inflow and price, and with a different annual water inflow (very dry, dry, normal, wet and very wet). The five weekly profiles of hourly water inflow were selected by clustering the historical series. The weekly profile of hourly energy price was formed with the average hourly prices of the historical series. It is easily seen in Fig. 4.9 that the trend reported in the literature (increasing water value with decreasing $\mathrm{V}$ ) disappears and even inverts as the annual water inflow decreases.

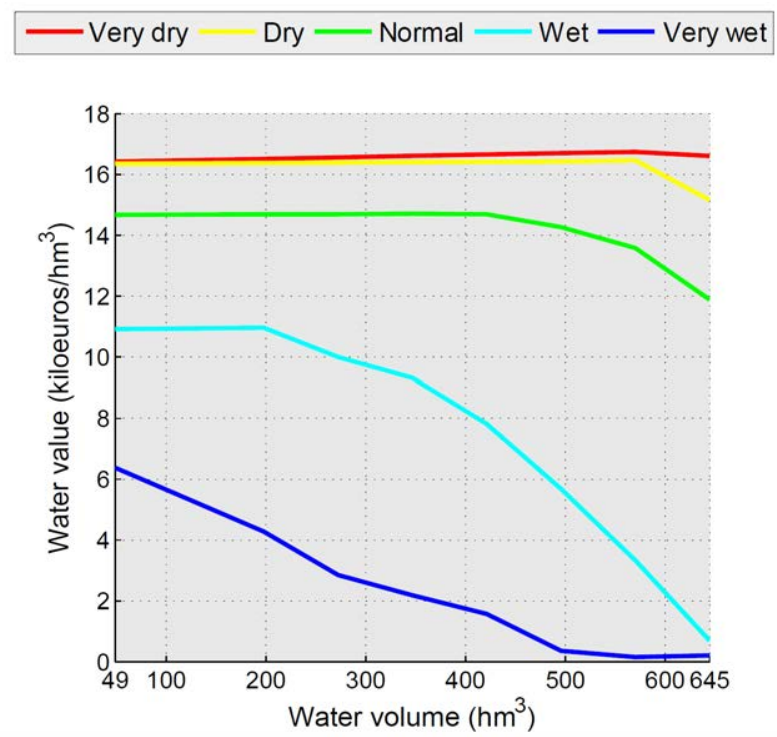

Figure 4.9.: Average profiles of water value for five different, and constant through the year, weekly water inflows (Very dry=lowest cluster; Dry=second lowest cluster; Normal=medium cluster; Wet=second highest cluster; Very wet=highest cluster).

The level curves of the flow values according to $\mathrm{F}$ across the year for three different $\rho$ (24 $\mathrm{h} ; 48 \mathrm{~h} ; 72 \mathrm{~h}$ ) at two different $\mathrm{V}$ (near the plant limits) are depicted in Fig. 4.10. This figure shows how the flow values are function of V, F and the moment of the year (see Eq.(4.23)). First, the variations were stronger at high V (left column of Fig. 4.10) than at low V (right column of Fig. 4.10); this is reasonable due to the expected revenue increase with V. Second, 
the highest values were located at low F; this is also expected since the highest slopes of the performance curves are located at low F (see in Fig. B.6) and, furthermore, because the beginning of the stage was selected at midnight when energy prices are usually lower. Third, and finally, the highest averages took place at the wettest moments of the year; as above-discussed in Fig. 4.6.

Several correlation analyses of the obtained flow values with different factors were performed with the aim of identifying any factor able to explain the seasonality of these values. Unfortunately, poor correlations were found in these trials; the highest ones being those obtained with the factors in the following expression:

$$
\text { factor }_{\rho}=\frac{\sum_{t=1}^{\rho-1}\left|p_{t}-p_{t+1}\right|}{\sum_{t=1}^{t_{m a x}}\left(f_{c 1} \cdot w_{t}\right)}
$$

These best found correlation factors with their correlations at $\mathrm{V}=347 \mathrm{hm}^{3}$ (in the middle of the reservoir of plant I) in terms of the evaluated $\rho$ and $F$ are gathered in Tab. 4.3.

Table 4.3.: Best correlation factors and their correlations with the flow values at $\mathrm{V}=347$ $\mathrm{hm}^{3}$ of the plant I in terms of $\rho$ and F.

\begin{tabular}{|c|c|c|c|c|c|c|c|}
\hline \multirow{2}{*}{$\begin{array}{c}\text { INTERVAL OF } \\
\text { THE PLANT FLOW }\end{array}$} & \multicolumn{7}{|c|}{ BEST FACTOR AND CORRELATION FOR $\rho$ OF } \\
\hline & $6 \mathrm{~h}$ & $12 \mathrm{~h}$ & $24 \mathrm{~h}$ & $36 \mathrm{~h}$ & $48 \mathrm{~h}$ & $60 \mathrm{~h}$ & $72 \mathrm{~h}$ \\
\hline $\begin{array}{c}\text { From } 0 \mathrm{~m}^{3} / \mathrm{s} \\
\text { to } 20 \mathrm{~m}^{3} / \mathrm{s}\end{array}$ & $\begin{array}{c}\text { factor }_{7} \\
-0.19 \\
\end{array}$ & $\begin{array}{l}\text { factor }_{6} \\
-0.20\end{array}$ & $\begin{array}{c}\text { factor }_{12} \\
0.08\end{array}$ & $\begin{array}{c}\text { factor }_{4} \\
0.24 \\
\end{array}$ & $\begin{array}{l}\text { factor }_{6} \\
0.06\end{array}$ & $\begin{array}{l}\text { factor }_{12} \\
-0.12\end{array}$ & $\begin{array}{l}\text { factor }_{72} \\
-0.32\end{array}$ \\
\hline $\begin{array}{c}\text { From } 20 \mathrm{~m}^{3} / \mathrm{s} \\
\text { to } 40 \mathrm{~m}^{3} / \mathrm{s}\end{array}$ & $\begin{array}{c}\text { factor }_{6} \\
0.18\end{array}$ & $\begin{array}{l}\text { factor }_{24} \\
-0.07\end{array}$ & $\begin{array}{c}\text { factor }_{6} \\
-0.11\end{array}$ & $\begin{array}{l}\text { factor }_{6} \\
-0.06\end{array}$ & $\begin{array}{c}\text { factor }_{12} \\
0.14\end{array}$ & $\begin{array}{c}\text { factor }_{24} \\
-0.06\end{array}$ & $\begin{array}{l}\text { factor }_{6} \\
-0.21\end{array}$ \\
\hline $\begin{array}{c}\text { From } 40 \mathrm{~m}^{3} / \mathrm{s} \\
\text { to } 100 \mathrm{~m}^{3} / \mathrm{s}\end{array}$ & $\begin{array}{l}\text { factor }_{12} \\
-0.11\end{array}$ & $\begin{array}{c}\text { factor }_{6} \\
0.09\end{array}$ & $\begin{array}{l}\text { factor }_{6} \\
-0.08\end{array}$ & $\begin{array}{l}\text { factor }_{6} \\
-0.22\end{array}$ & $\begin{array}{l}\text { factor }_{6} \\
-0.63\end{array}$ & $\begin{array}{l}\text { factor }_{6} \\
-0.34\end{array}$ & $\begin{array}{l}\text { factor }_{6} \\
-0.59\end{array}$ \\
\hline $\begin{array}{c}\text { From } 100 \mathrm{~m}^{3} / \mathrm{s} \\
\text { to } 160 \mathrm{~m}^{3} / \mathrm{s}\end{array}$ & $\begin{array}{c}\text { factor }_{6} \\
0.03\end{array}$ & $\begin{array}{c}\text { factor }_{72} \\
0.09\end{array}$ & $\begin{array}{l}\text { factor }_{6} \\
-0.20\end{array}$ & $\begin{array}{l}\text { factor }_{24} \\
-0.49\end{array}$ & $\begin{array}{l}\text { factor }_{72} \\
-0.04\end{array}$ & $\begin{array}{l}\text { factor }_{12} \\
-0.02\end{array}$ & $\begin{array}{l}\text { factor }_{12} \\
-0.12\end{array}$ \\
\hline $\begin{array}{c}\text { From } 160 \mathrm{~m}^{3} / \mathrm{s} \\
\text { to } 219 \mathrm{~m}^{3} / \mathrm{s}\end{array}$ & $\begin{array}{c}\text { factor }_{12} \\
0.06\end{array}$ & $\begin{array}{c}\text { factor }_{6} \\
-0.43\end{array}$ & $\begin{array}{c}\text { factor }_{6} \\
-0.12\end{array}$ & $\begin{array}{c}\text { factor }_{60} \\
0.21\end{array}$ & $\begin{array}{l}\text { factor }_{6} \\
-0.03\end{array}$ & $\begin{array}{l}\text { factor }_{6} \\
-0.16\end{array}$ & $\begin{array}{c}\text { factor }_{48} \\
0.14\end{array}$ \\
\hline $\begin{array}{c}\text { From } 219 \mathrm{~m}^{3} / \mathrm{s} \\
\text { to } 279 \mathrm{~m}^{3} / \mathrm{s}\end{array}$ & $\begin{array}{c}\text { factor }_{24} \\
0.06\end{array}$ & $\begin{array}{c}\text { factor }_{6} \\
0.16\end{array}$ & $\begin{array}{c}\text { factor }_{6} \\
0.25\end{array}$ & $\begin{array}{c}\text { factor }_{24} \\
0.22\end{array}$ & $\begin{array}{c}\text { factor }_{24} \\
0.32\end{array}$ & $\begin{array}{c}\text { factor }_{24} \\
0.36\end{array}$ & $\begin{array}{l}\text { factor }_{6} \\
-0.03\end{array}$ \\
\hline
\end{tabular}




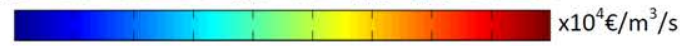

(a)

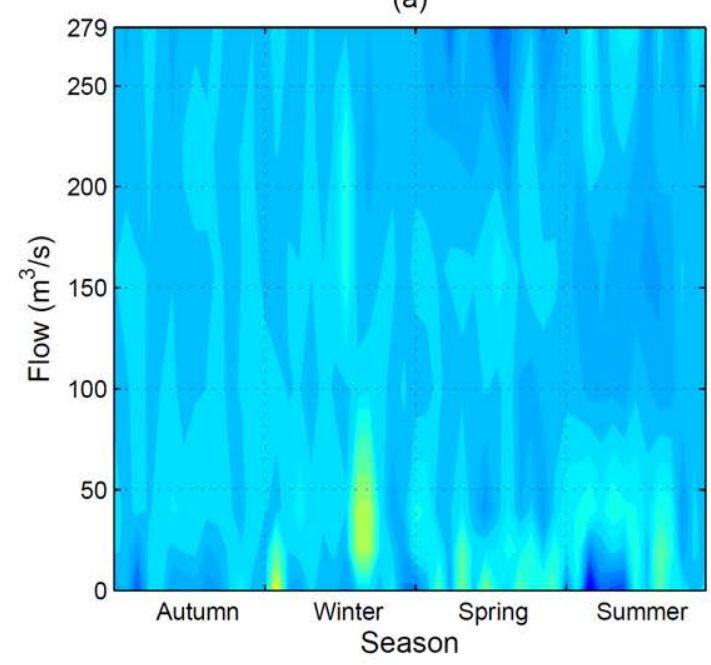

(c)

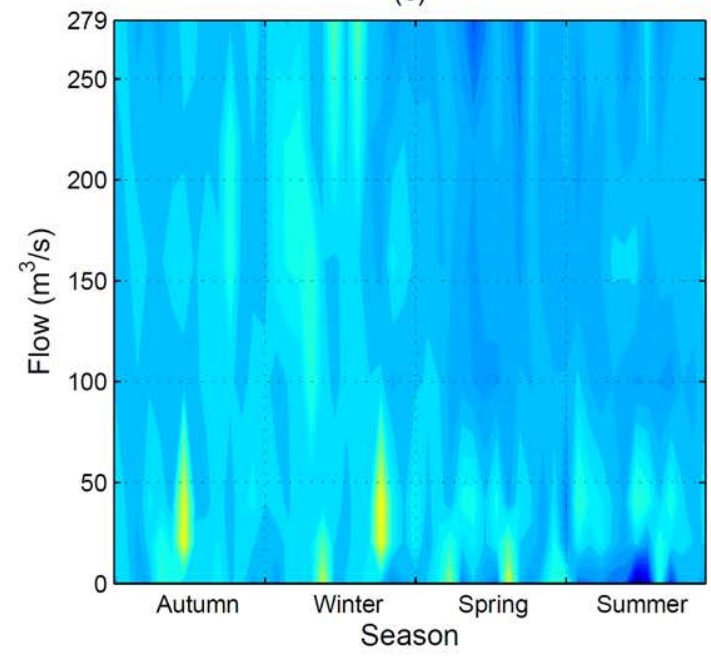

(e)

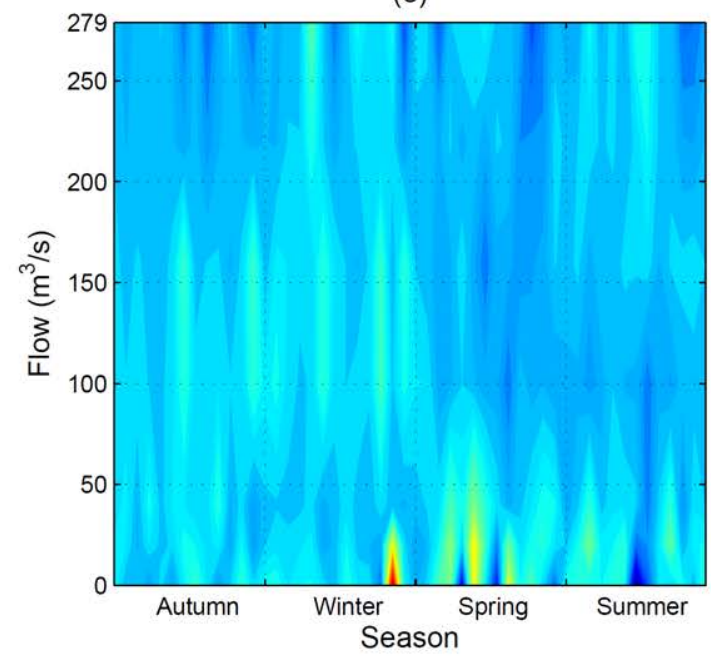

(b)

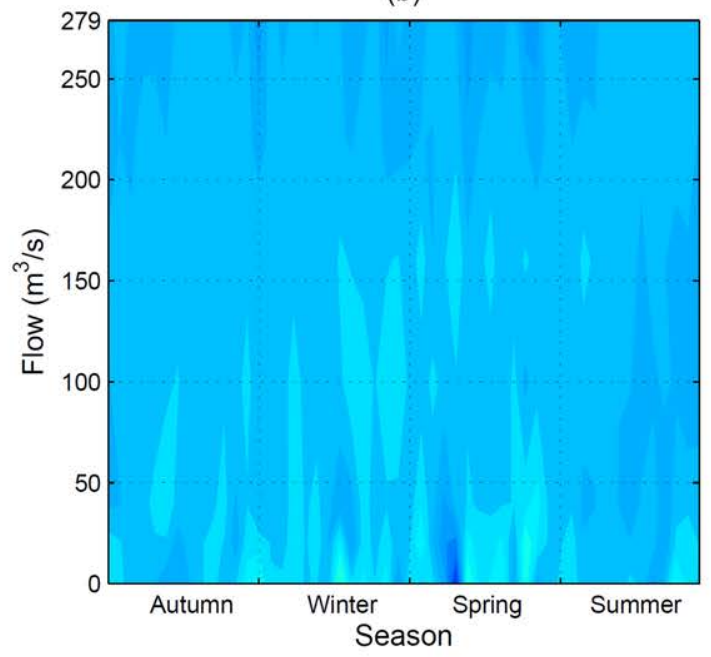

(d)

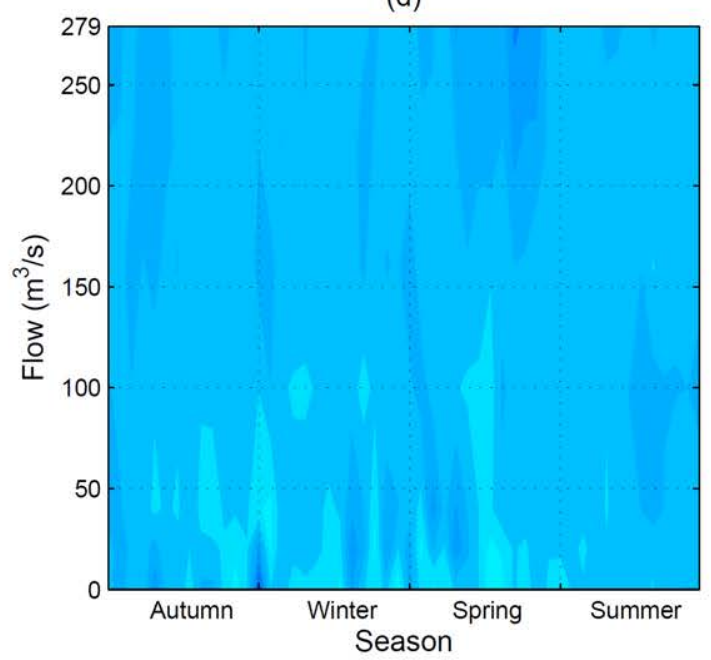

(f)

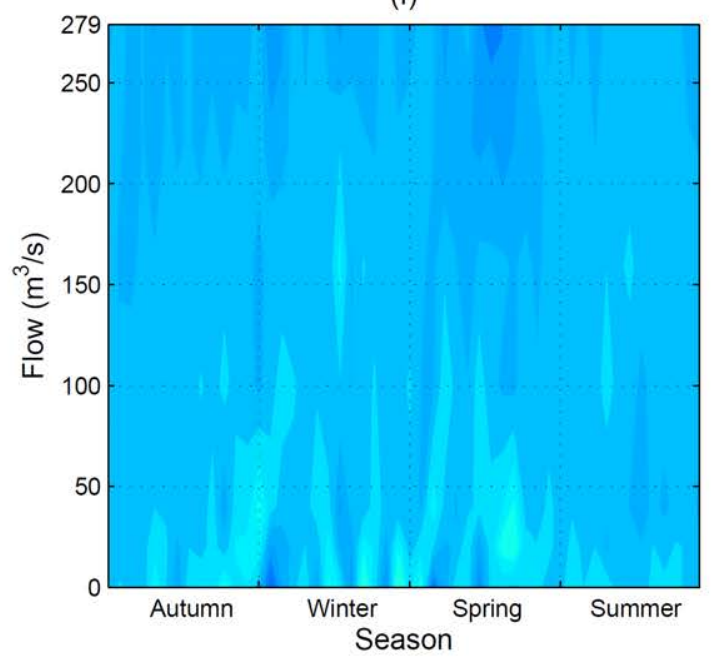

Figure 4.10.: Flow value level curves of the plant I with: (a) $\rho=24 \mathrm{~h}$ at $\mathrm{V}=570 \mathrm{hm}^{3}$; (b) $\rho=24 \mathrm{~h}$ at $\mathrm{V}=123 \mathrm{hm}^{3}$; (c) $\rho=48 \mathrm{~h}$ at $\mathrm{V}=570 \mathrm{hm}^{3}$; (d) $\rho=48 \mathrm{~h}$ at $\mathrm{V}=123 \mathrm{hm}^{3}$; (e) $\rho=72 \mathrm{~h}$ at $\mathrm{V}=570 \mathrm{hm}^{3}$; (f) $\rho=72 \mathrm{~h}$ at $\mathrm{V}=123 \mathrm{hm}^{3}$. 


\subsection{Optimisation models for long-term hydro scheduling subject to environmental constraints (part I)}

This study has three objectives. The main objective is the first one of the thesis (as discussed in sec. 1.3) that matches with the title of the current section. The secondary objectives are to check again the validity of the formulae (3.1-3.3) as well as to corroborate some of the findings of the study 3 related to the generated energy, operating hours, price tracking, spillage volume, and start-ups and shut-downs (as discussed in sec. 3.3.3).

\subsubsection{Methodology}

In the previous study, the water and flow values of a peak hydropower plant were calculated subject to different magnitudes of $\varphi$ and $\rho$. The usefulness of those calculations only can be evaluated by means of simulations [Doorman (2009)]. Therefore, three sets of simulations were carried out in this study in order to select the best approach for a long-term hydro scheduling model.

The first approach, what might be called the classical approach or WV00, consists in neglecting the effects of $\varphi$ and $\rho$ on the long-term scheduling by using in all the simulations the water values obtained in the scenario without environmental constraints. The second approach, $W V \varphi \rho$, is based on the assumption that the long-term influence of $\varphi$ and $\rho$ can be adequately modelled by the water value calculated in each considered scenario with the model modVstu4 (as discussed in sec.4.1.1). Finally, the third approach, WEFV $\varphi \rho$, uses the corresponding water and flow values calculated in each considered scenario with the model modVFstu4 (as discussed in sec.4.1.1).

Two models were developed to perform the simulations in this study; both based on deterministic mixed LP and subsequent recalculations of the power outputs and the resulting revenue from the obtained decisions with the real generation characteristic of the plant. One model $(\operatorname{sim} W V)$ was used for $W V 00$ and $W V \varphi \rho$, and the other $(\operatorname{sim} W \mathscr{E} F V)$ for $W \mathscr{E} F V \varphi \rho$.

As in the study 4, water inflow, energy price and evaporation rate were considered as input variables. In this study, three synthetic series (S1, S2 and S3) were built through the Markov chains of these input variables with a length of a decade.

In order to make the calculations easier and given the length of the used stage (as discussed in sec.1.2), a weekly perfect forecast of all input variables was assumed, the same $\mathrm{V}$ was imposed at the beginning of each simulation series, and the results obtained with the water value of the problem without environmental constraints was used as a comparison standard. Given the coarse grid used to obtain the water and flow values, the applied mixed LP formulations of these values were built from spline interpolations of their obtained values (21 states for both $\mathrm{V}$ and $\mathrm{F}$ ) with the aim of increasing the accuracy (as discussed in sec. 2.2.2). 


\section{- Description of SimWV}

The purpose of $\operatorname{sim} W V$, Eqs. (A.19, A.22, A.25-A.26, A.28-A.29, A.32, A.47-A.48, A.50-A.58, A.61-A.71 and A.73-A.84), is to obtain the optimal hourly operation of a peak hydropower plant through a weekly planning period, considering $W V 00$ or $W V \varphi \rho$. It is an improved version of the LP module of modVstu4 (as discussed in sec. 4.1.1) whose only difference from that model is the use of up to two performance curves per subproblem instead of one, Eq. (A.52).

\section{- Description OF SIMW\&FV}

The purpose of $\operatorname{sim} W E F V$, Eqs. (A.20, A.22, A.25-A.26, A.28-A.32, A.40-A.41, A.47-A.48, A.50-A.58, A.61-A.71, A.73-A.79 and A.85-A.95), is the same as that of $\operatorname{sim} W V$ but in this case considering $W \mathscr{E} F V \varphi \rho$. $\operatorname{sim} W \mathscr{E} F V$ is an improved version of the LP module of modVFstu4 (as discussed in sec. 4.1.1) and, again, its only difference from that model is the use of up to two performance curves per subproblem instead of one, Eq. (A.52).

\subsubsection{Case study}

Plant B (Tab. B.2, Fig. B.17 and Fig. B.19(f)), located in the northwest of Spain (Fig. B.1), was the peak hydropower plant selected for carrying out the current study with the aim of checking the validity of the formulae (3.1-3.3) since this plant was the one with the worst results among those tested in the study 3 (as discussed in sec. 3.3.3). Therefore, the water and flow values of this plant were calculated with the same models used in the study 4, modVstu4 and modVFstu4 (as discussed in sec.4.1.1), prior to the simulations.

The daily water inflows and the hourly energy prices were again taken from the web page of the Centre for Public Works Studies and Experimentation [CEDEX (2013)] and from the web page of the Iberian Electricity Market Operator [OMIE (2011)], respectively. The weekly evaporation rates were again estimated by application of the empirical temperaturebased formula proposed in Dragoni and Valigi (1994) to the average monthly temperatures taken from the web page of Spanish Statistical Office [INE (1960)]. The analysed scenarios of the input variables as well as the evaluated magnitudes of $\varphi$ and $\rho$, expressed in relative terms following the methodology of the study 1 (as discussed in sec. 3.1.1), are included in Tab. 4.4.

The above-described models (sec. 4.2.1) were implemented in MATLAB ${ }^{\circledR}$, each subproblem being solved using CPLEX ${ }^{\mathrm{TM}}$ under GAMS ${ }^{\mathrm{TM}}$, on 16 threads (used each pair in parallel [Baslis and Bakirtzis (2011)]) of an Intel ${ }^{\circledR}$ Xeon ${ }^{\circledR}$ E5 at $3.1 \mathrm{GHz}$ and 64 GB RAM running on Microsoft Windows ${ }^{\circledR}$. Once more, following the suggestion in Carvalho and Pinto (2006), a small sensitivity analysis of the performance of the models was done using as a basis the results of those carried out in the previous studies. The selected parameters are given in Tab. 4.5.

A total of 18 estimations of water and flow values were undertaken in this study; 11 considering only $\mathrm{V}$ and 7 considering $\mathrm{V}$ and $\mathrm{F}$. These estimations were decomposed into 2,446,080 
short-term subproblems: 687,960 were solved by modVstu4 and 1,755,120 by modVFstu4. After that, a total of 84 long-term simulations were carried out; 33 considering WV00, 30 under $W V \varphi \rho$ and 21 under $W \mathscr{E} F V \varphi \rho$. These simulations were decomposed into 43,680 short-term subproblems: 32,760 were solved by $\operatorname{sim} W V$ and 10,920 by $\operatorname{sim} W \mathscr{G} F V$.

Table 4.4.: Input variable scenarios and environmental constraints used in the study 5 .

\begin{tabular}{|c|c|c|}
\hline CONCEPT & SCENARIOS & DESCRIPTION \\
\hline \hline Water inflows & 5 Markov classes of lag 1 & Hourly \\
\hline Energy prices & 3 Markov classes of lag 1 & Hourly \\
\hline Evaporation rates & Average & Weekly \\
\hline & $(0 \%, 0 \mathrm{~h}) ;(1 \%, 0 \mathrm{~h}) ;$ & $\varphi_{\text {Oct. }- \text { Dec. }}=0.75 \varphi ;$ \\
& $(5 \%, 0 \mathrm{~h}) ;(9 \%, 0 \mathrm{~h}) ;$ & $\varphi_{\text {Jan. }- \text { Mar. }}=1.75 \varphi ;$ \\
& $(0 \%, 24 \mathrm{~h}) ;(0 \%, 48 \mathrm{~h}) ;$ & $\varphi_{\text {Apr.-Jun. }}=1.20 \varphi ;$ \\
& $(0 \%, 72 \mathrm{~h}) ;(1 \%, 24 \mathrm{~h}) ;$ & $\varphi_{\text {Jul.-Sep. }}=0.30 \varphi ;$ \\
& $(5 \%, 48 \mathrm{~h}) ;(9 \%, 72 \mathrm{~h}) ;$ & $\rho_{1-k^{\text {max }}}^{u p}=0.75 \rho ;$ \\
& $(3.97 \%, 133.58 \mathrm{~h})^{a}$ & $\rho_{1-k^{\max }}^{\text {dow }}=1.50 \rho$ \\
\hline
\end{tabular}

${ }^{a}$ Planned to be imposed by the water basin authority.

Table 4.5.: Characteristic parameters of the LP algorithms of the study 5 .

\begin{tabular}{|c|c|c|}
\hline MODELS & PARAMETER & VALUE \\
\hline \hline modVstu4 & Relative optimality criterion (\%) & 0.1 \\
\cline { 2 - 3 } modVFstu4 & Time limit for each subproblem $(\mathrm{s})$ & 20 \\
\hline \multirow{3}{*}{$\operatorname{sim} W V$} & Maximum number of performance curves $\left(\overline{c^{m a x}}\right)$ & 2 \\
\cline { 2 - 3 } $\operatorname{sim} W \& F V$ & Gross head "covered" by each performance curve $\left(H^{\%}\right)$ & 10 \\
\cline { 2 - 3 } & Relative optimality criterion (\%) & $0.1^{a}$ \\
\cline { 2 - 3 } & Time limit for each subproblem (s) & 200 \\
\hline
\end{tabular}

${ }^{a}$ As recommended in Fu and Shahidehpour (2007)

\subsubsection{Main results and discussion}

The variations relative to $W V 00$ calculated by $\operatorname{sim} W V$, in terms of $\varphi$ or $\rho$, of the aggregate revenue calculated by $\operatorname{sim} W V$ under $W V \varphi \rho$ and by $\operatorname{sim} W \mathscr{E} F V$ under $W \mathscr{G} F V \varphi \rho$ plus the future expected revenue according to $W V 00$ corresponding to the final volume obtained in each simulation of each synthetic series (S1, S2 and S3) are drawn in Fig. 4.11. This figure seems to indicate that $W V \varphi \rho$ was more convenient for scenarios with only $\varphi$, whereas $W V 00$ was more suitable for those with only p. Moreover, Fig. 4.11(b) shows a very bad performance of $W E F V \varphi \rho$. As $\varphi$ must be present in all natural flows of the river [Sanguedo Baptista and De Aquino Galeano Massera Da Hora (2013)], it was imposed to be lower than or equal to the current water inflow into the reservoir (Eq.(A.22)) and this is the reason why Fig. 4.11(a) shows results for $\varphi=4.6 \%$ and $\varphi=7.6 \%$ instead of $\varphi=5 \%$ and $\varphi=9 \%$. This circumstance is repeated in the following figures and tables. 

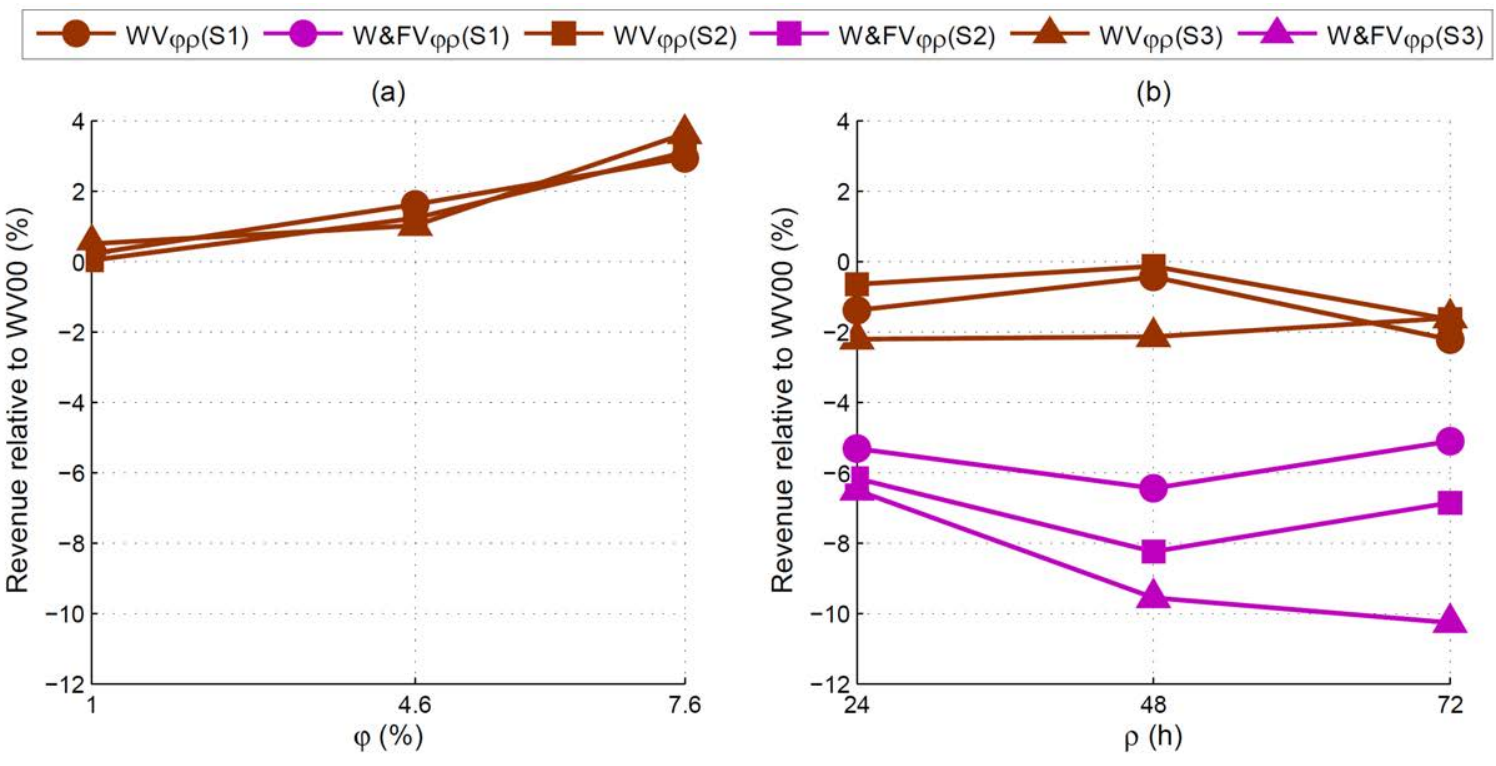

Figure 4.11.: Revenue of the plant $\mathrm{B}$ in the study 5 relative to $W V 00$ of: (a) $W V \varphi \rho$ in terms of $\varphi ;($ b) both $W V \varphi \rho$ and $W \xi F V \varphi \rho$ in terms of $\rho$.

In Tab.4.6, the variations relative to the $W V 00$ calculated by $\operatorname{sim} W V$ of the aggregate revenue calculated by $\operatorname{sim} W V$ under $W V \varphi \rho$ and by $\operatorname{sim} W E F V$ under $W E F V \varphi \rho$ plus the future expected revenue according to $W V 00$ corresponding to the final volume obtained in each simulation of each synthetic series (S1, S2 and S3) for the scenarios with combined $\varphi$ and $\rho$ are presented. The results seem to point out that $W V \varphi \rho$ was more adequate for this kind of scenarios characterised by the presence of both $\varphi$ and $\rho$. Once more, the performance of $W \mathscr{E} F V \varphi \rho$ was the worst of all approaches.

Table 4.6.: Revenue of the plant B in the study 5 under the scenarios with combined $\varphi$ and $\rho$ relative to $W V 00$ of both $W V \varphi \rho$ and $W \mathscr{E} F V \varphi \rho$.

\begin{tabular}{|c|c|c|c|c|c|c|c|}
\hline \multicolumn{2}{|c|}{ SCENARIO WITH } & \multicolumn{6}{|c|}{ REVENUE RELATIVE TO $W V 00(\%)$ OF } \\
\hline $\begin{array}{c}\varphi \\
(\%)\end{array}$ & $\rho$ & \multicolumn{2}{c|}{$W V \varphi \rho$ FOR THE SERIES } & \multicolumn{2}{c|}{$W \xi F V \varphi \rho$ FOR THE SERIES } \\
\cline { 3 - 8 } & $(\mathrm{h})$ & $\mathrm{S} 1$ & $\mathrm{~S} 2$ & $\mathrm{~S} 3$ & $\mathrm{~S} 1$ & $\mathrm{~S} 2$ & $\mathrm{~S} 3$ \\
\hline \hline 1 & 24 & 0.1 & 0.4 & -0.9 & -3.8 & -5.7 & -9.6 \\
\hline 4.6 & 48 & 1.3 & 0.3 & 0.1 & -1.9 & -5.4 & -9.2 \\
\hline 7.6 & 72 & 1.1 & 3.0 & 0.6 & -0.2 & -1.6 & -4.5 \\
\hline 3.7 & 133.58 & 0.9 & 2.5 & -0.6 & -3.2 & -1.2 & -10.1 \\
\hline
\end{tabular}

The long-term economic impacts of several values of $\varphi$ or $\rho$ on plant B deterministically calculated in study 3 (as discussed in sec. 3.2) and those determined through simulations of each synthetic series (S1, S2 and S3) considering $W V 00, W V \varphi \rho$ and $W \mathscr{E} F V \varphi \rho$ in this study are compared with those predicted by the formulae (3.1-3.2) in Fig. 4.12. As it can be seen, most of the simulations fell well within the confidence interval of the formulae. Only the results obtained considering $W \mathscr{E} F V \varphi \rho$, because of its bad performance, and those calculated by $W V \varphi \rho$ for the scenario with $\varphi=7.6 \%$, as it happened $\varphi=9 \%$ in the study 3 (see in Fig. 3.7(b)), were out the confidence interval of the formulae. 


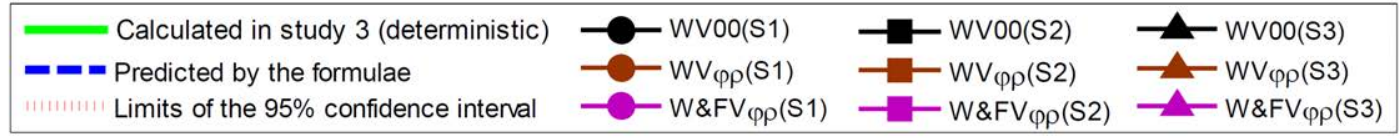

(a)

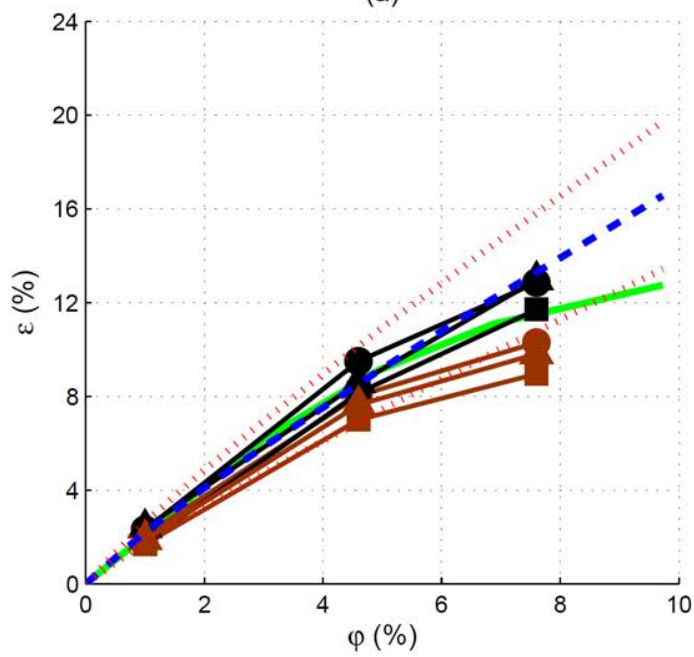

(b)

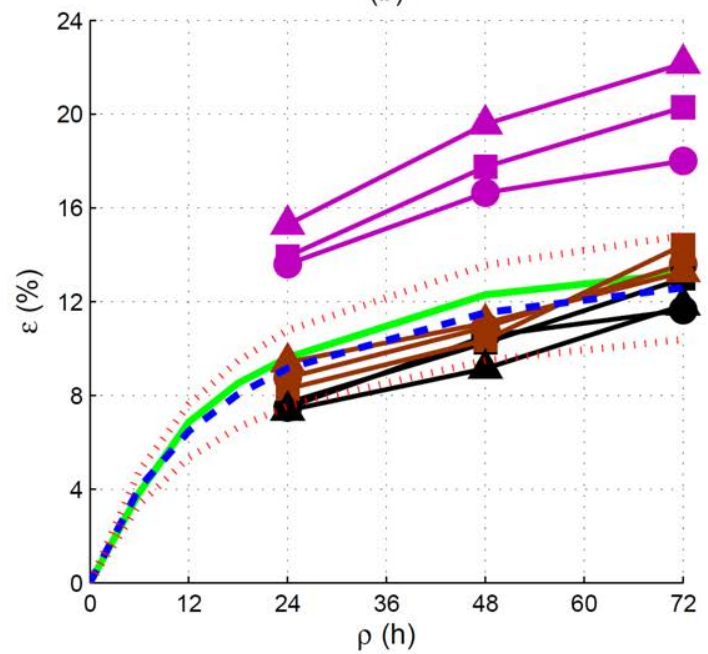

Figure 4.12.: Long-term economic impacts of the plant B deterministically calculated in study 3 , those determined in study 5 through simulation considering $W V 00, W V \varphi \rho$ and $W \& \xi F V \varphi \rho$, and those predicted by the formulae (3.1-3.2) in terms of: (a) $\varphi$; (b) $\rho$.

Regarding the formula (3.3), the long-term economic impacts of the analysed scenarios with combined $\varphi$ and $\rho$ on plant B predicted by this formula and those determined through simulations of each synthetic series (S1, S2 and $\mathrm{S} 3$ ) considering $W V 00, W V \varphi \rho$ and $W E F F \varphi \rho$ in this study are exposed in Tab. 4.7. This time, a third of the results obtained by WV00, a sixth of those by $W V \varphi \rho$ and all by $W \mathscr{E} F V \varphi \rho$ did not fall within the confidence interval of the considered formula. It is worth pointing out that $\rho$ in the last scenario (planned by the river basin authority) is far above the highest one used in the sample for the calculation of the formula (3.3) (see in Tab. 3.6).

Finally, the checking of the findings of the study 3 (see in Fig. 3.10 and Fig. 3.11) was carried out with the policies obtained with the highest revenue, which were the $W V \varphi \rho$ 's ones for scenarios with $\varphi$ and the WV00's ones for those without $\varphi$ (see in Fig. 4.12). Thus, Fig. 4.13 shows the averages power · price-duration curves across all simulations of plant B in terms of $\varphi$ and $\rho$ corresponding to the scenarios in which only $\varphi$ or $\rho$ are present. As in the study 3 (see in sec. 3.3.3), the larger $\varphi$ or $\rho$, the worse the price tracking.

In Fig. 4.14 are drawn the averages variations across al simulations relative to the problem without environmental constraints of the generated energy, number of operating hours, spillage volumes and start-ups and shut-downs of the case study in the same terms of the previous figure. Again as in the study 3 , as $\varphi$ increased, the spillages grew and the generated energy and the number of start-ups and shut-downs decreased; as $\rho$ increased, the operating hours and spillages grew, whereas the number of start-ups and shut-downs decreased. 
4.2 Optimisation models for long-term hydro scheduling subject to environmental constraints (part I)

Table 4.7.: Long-term economic impacts of the scenarios with combined $\varphi$ and $\rho$ on plant B determined in study 5 through simulation considering $W V 00, W V \varphi \rho$ and $W \mathscr{E} F V \varphi \rho$, and those predicted by the formula (3.3).

\begin{tabular}{|c|c|c|c|c|c|c|}
\hline \multirow{2}{*}{\multicolumn{2}{|c|}{$\begin{array}{c}\text { SCENARIO } \\
\text { WITH }\end{array}$}} & \multirow{4}{*}{ SERIES } & \multicolumn{4}{|c|}{$\varepsilon(\%)$} \\
\hline & & & \multirow{2}{*}{\multicolumn{3}{|c|}{$\begin{array}{l}\text { DETERMINED WITH THE } \\
\text { SIMULATION APPROACH }\end{array}$}} & \multirow{3}{*}{$\begin{array}{c}\text { PREDICTED BY } \\
\text { THE FORMULA (3.3) WITHIN } \\
\text { THE CONFIDENCE INTERVAL }\end{array}$} \\
\hline \multirow{2}{*}{$\begin{array}{l}\varphi \\
(\%) \\
\end{array}$} & \multirow{2}{*}{$\begin{array}{l}\rho \\
\text { (h) }\end{array}$} & & & & & \\
\hline & & & WV00 & $W V \varphi \rho$ & $W \mathscr{E} F V \varphi \rho$ & \\
\hline \multirow{3}{*}{1} & \multirow{3}{*}{24} & $\mathrm{~S} 1$ & 11.0 & 10.8 & 14.3 & \multirow{3}{*}{$11.5[9.5 ; 13.5]$} \\
\hline & & $\mathrm{S} 2$ & 9.8 & 9.4 & 14.9 & \\
\hline & & S3 & 9.1 & 9.9 & 17.9 & \\
\hline \multirow{3}{*}{4.6} & \multirow{3}{*}{48} & S1 & 18.6 & 17.5 & 20.2 & \multirow{3}{*}{$16.9[14.0 ; 19.9]$} \\
\hline & & S2 & 17.4 & 17.2 & 21.8 & \\
\hline & & S3 & 16.7 & 16.6 & 24.4 & \\
\hline \multirow{3}{*}{7.6} & \multirow{3}{*}{72} & $\mathrm{~S} 1$ & 22.0 & 21.2 & 22.2 & \multirow{3}{*}{$18.8[15.5 ; 22.0]$} \\
\hline & & $\mathrm{S} 2$ & 22.5 & 20.2 & 23.8 & \\
\hline & & S3 & 21.3 & 20.9 & 24.8 & \\
\hline \multirow{3}{*}{3.7} & \multirow{3}{*}{133.58} & $\mathrm{~S} 1$ & 21.8 & 21.1 & 24.3 & \multirow{3}{*}{$18.2[15.0 ; 21.3]$} \\
\hline & & $\mathrm{S} 2$ & 24.8 & 22.8 & 25.7 & \\
\hline & & S3 & 19.7 & 20.1 & 27.7 & \\
\hline
\end{tabular}

(a)

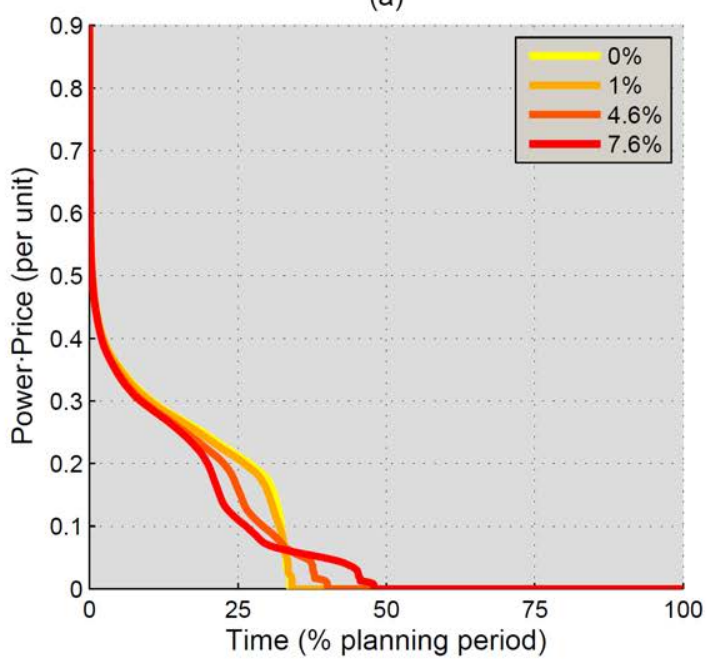

(b)

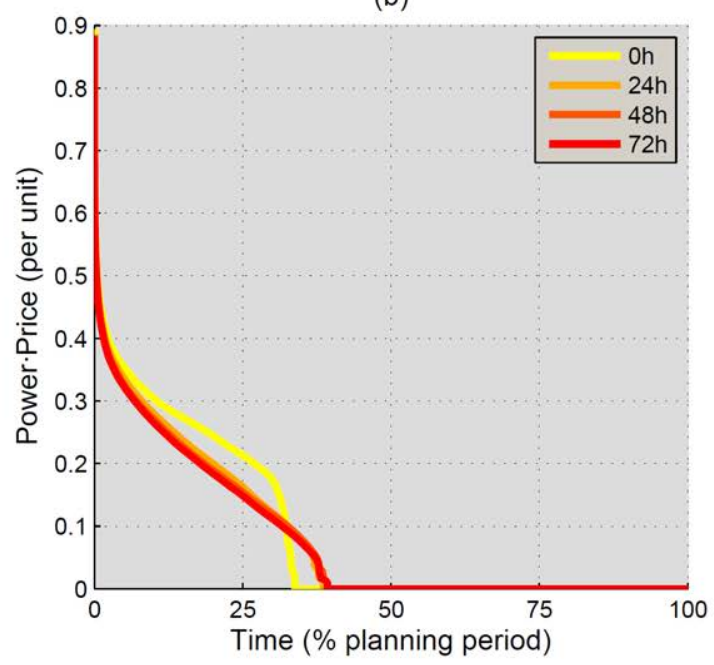

Figure 4.13.: Average power - price-duration curves across all simulations of the plant $B$ in terms of: (a) $\varphi$ under $W V \varphi \rho$; (b) $\rho$ f under $W V 00$. 

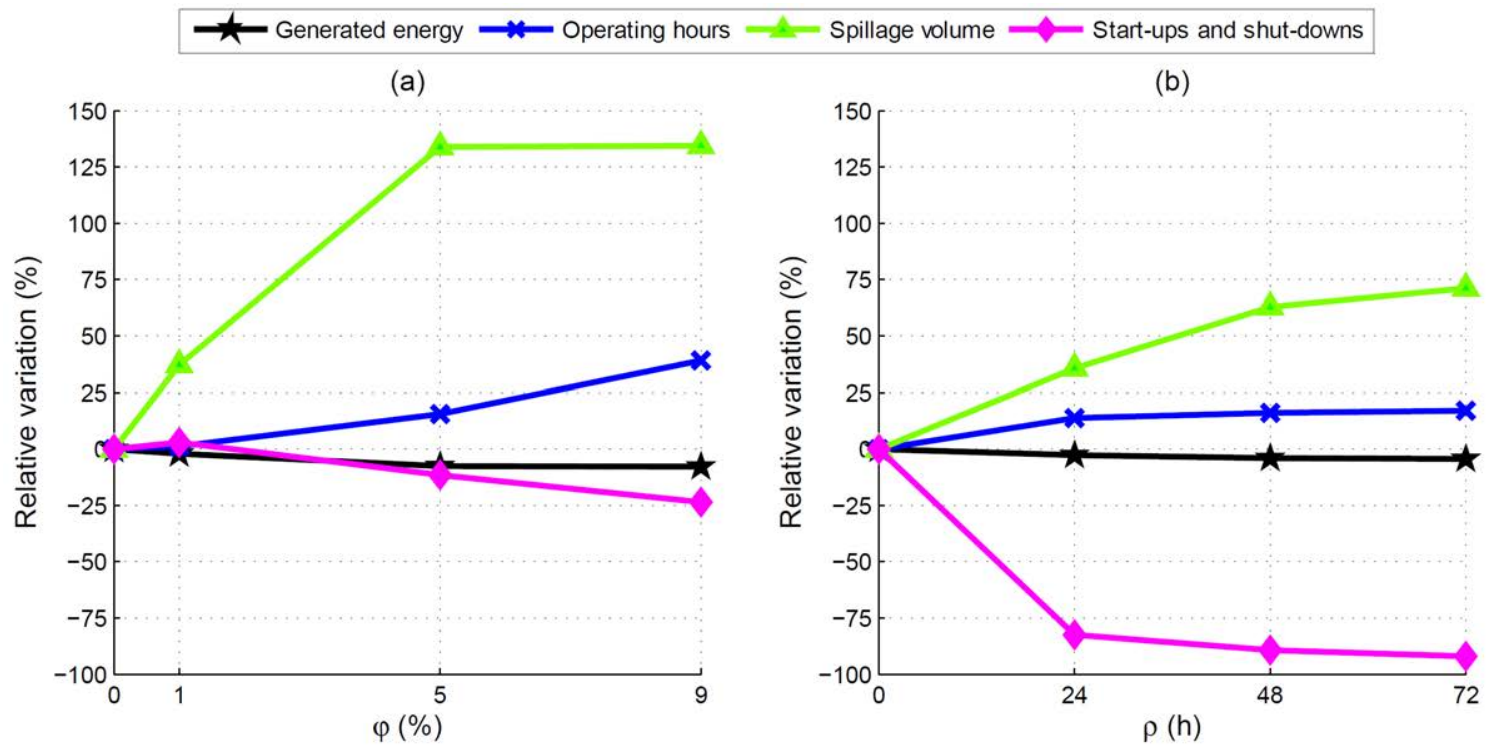

Figure 4.14.: Average variations across all simulations relative to the problem without environmental constraints of the generated energy, operating hours, spillage volume and start-ups and shut-downs of the plant B in terms of: (a) $\varphi$ under $W V \varphi \rho$; (b) $\rho$ under WV00.

\subsection{Optimisation models for long-term hydro scheduling subject to environmental constraints (part II)}

This is the final study of the thesis and it was fully focused on the achievement of its first objective. It is a natural continuation of the previous study, providing insight into the performance of the best approaches found in that one. The idea of the current study was to confirm the validity of the results obtained in the study 5 (as discussed in sec. 4.2.3) on the convenience of considering $\varphi$ and $\rho$ for the determination of water value.

The results obtained in the study 5 suggest that considering $\varphi$ or $\rho$ for the determination of water value provides, respectively, better or worse results than the classical approach which neglects $\varphi$ and $\rho$ for the said determination. Given that the average flow of the Markov chain used to model the water inflow in the study $5\left(4.7 \mathrm{~m}^{3} / \mathrm{s}\right)$ differs, due to inherent limitations of the methodology used to build Markov chains (as discussed in sec. 2.3.1), from the historical average water inflow $\left(4.4 \mathrm{~m}^{3} / \mathrm{s}\right)$, which was also the average water inflow of the simulation series used in that study, a longer water inflow series with the same average flow of the applied Markov chain was used in this study to confirm that the mismatch between the average water inflows of the Markov chain and the simulation series did not lead to misleading results.

\subsubsection{Methodology}

The methodology used in this study is practically identical to the one applied in the study 5 (see in sec. 4.2.1). The differences between both studies are related to the considered synthetic series and are summarised in Tab. 4.8. 
4.3 Optimisation models for long-term hydro scheduling subject to environmental constraints (part II)

Table 4.8.: Differences between studies 5 and 6 .

\begin{tabular}{|c|c|c|}
\hline ConCEPT & StUdy 5 & StUdy 6 \\
\hline \hline Average flow series & Historical & Average flow of the Markov chain \\
\hline Number of series & 3 & 1 \\
\hline Length of the series & Decade & Century \\
\hline
\end{tabular}

\subsubsection{Case study}

The same case study, input data, scenarios and characteristic parameters of the LP algorithms applied in the study 5 were reutilised in this one (as discussed in sec. 4.2.2).

A total of 28 long-term simulations were carried out; 11 considering WV00, 10 under $W V \varphi \rho$ and 7 under $W \mathscr{E} F V \varphi \rho$. These simulations were decomposed into 145,600 short-term subproblems: 109,200 were solved by $\operatorname{sim} W V$ and 36,400 by $\operatorname{sim} W \mathscr{E} F V$.

\subsubsection{Main results and discussion}

The variations relative to $W V 00$ calculated by $\operatorname{sim} W V$, in terms of $\varphi$ or $\rho$, of the aggregate revenue calculated by $\operatorname{sim} W V$ under $W V \varphi \rho$ and by $\operatorname{sim} W \xi F V$ under $W \xi F V \varphi \rho$ plus the future expected revenue according to $W V 00$ corresponding to the final volume obtained in each simulation are drawn in Fig. 4.15. This figure seems to corroborate the results obtained in the previous study, that is, $W V \varphi \rho$ was more convenient for scenarios with only $\varphi$, whereas WV00 was more suitable for those with only $p$.

(a)

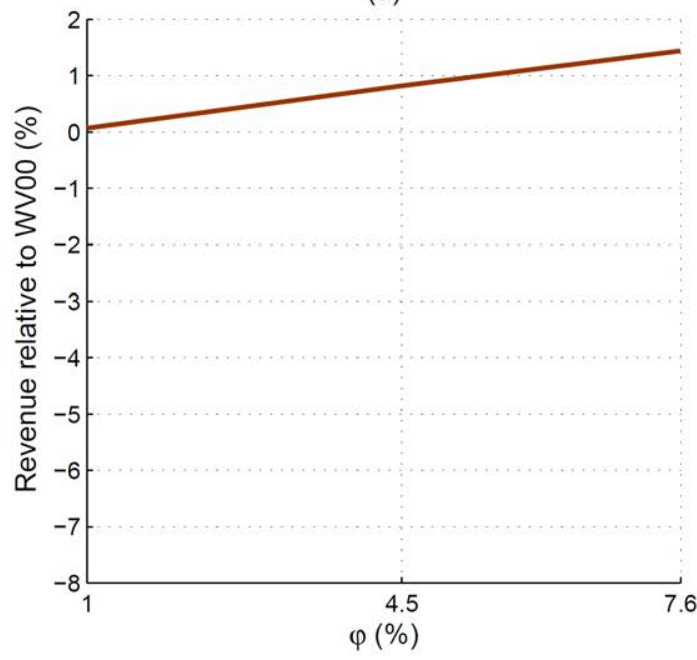

(b)

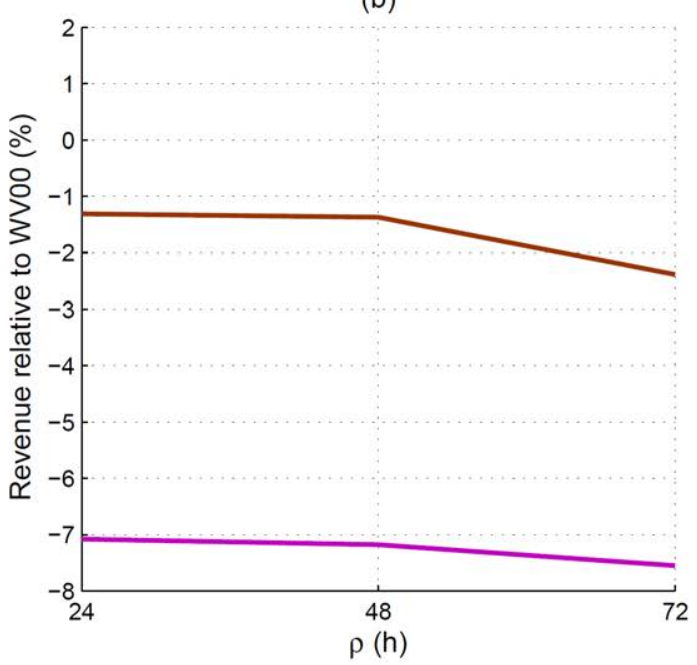

Figure 4.15.: Revenue of the plant $\mathrm{B}$ in the study 6 relative to $W V 00$ of: (a) $W V \varphi \rho$ in terms of $\varphi$; (b) both $W V \varphi \rho$ and $W \xi F V \varphi \rho$ in terms of $\rho$.

In Tab. 4.9, the variations relative to the $W V 00$ calculated by $\operatorname{sim} W V$ of the aggregate revenue calculated by $\operatorname{sim} W V$ under $W V \varphi \rho$ and by $\operatorname{sim} W \mathscr{E} F V$ under $W E F V \varphi \rho$ plus the future expected revenue according to $W V 00$ corresponding to the final volume obtained in 
each simulation for the scenarios with combined $\varphi$ and $\rho$ are presented. Unlike the study 5 , the results seem to point out that WV00 was more adequate for this kind of scenarios characterised by the presence of both $\varphi$ and $\rho$. Once more, the performance of $W \mathscr{E} F V \varphi \rho$ was the worst of all approaches.

Table 4.9.: Revenue of the plant B in the study 6 under the scenarios with combined $\varphi$ and $\rho$ relative to $W V 00$ of both $W V \varphi \rho$ and $W \mathscr{E} F V \varphi \rho$.

\begin{tabular}{|c|c|c|c|}
\hline \multicolumn{2}{|c|}{ SCEnario With } & \multicolumn{2}{c|}{ ReVEnUE RELATIVE } \\
\hline$\varphi$ & $\rho$ & \multicolumn{2}{|c|}{ To $W V 00(\%)$ OF } \\
\cline { 3 - 4 }$(\%)$ & $(\mathrm{h})$ & $W V \varphi \rho$ & $W \xi F V \varphi \rho$ \\
\hline \hline 1 & 24 & -1.2 & -6.4 \\
\hline 4.5 & 48 & -0.4 & -5.5 \\
\hline 7.6 & 72 & 0.5 & -2.7 \\
\hline 3.7 & 133.58 & -1.2 & -5.4 \\
\hline
\end{tabular}




\section{CONCLUSIONS}

This chapter sets out the main contributions and conclusions of the thesis and proposes some future research directions.

\subsection{Main contributions}

The main contributions of the thesis are the following:

- Two different long-term optimisation models for hydro scheduling subject to minimum flows and maximum ramping rates (developed through chapter 4): a model with one state variable (stored volume) and the other with two (stored volume and released flow). This contribution constitutes the first objective of the thesis.

- Several sensitivity analyses of the long-term effects of minimum flows and maximum ramping rates on certain economical and operational aspects of a peak hydropower plant, such as economic impact, generated energy, hydropower efficiency, net head, operating hours, price tracking, spillage volume, start-ups and shut-downs, and water and flow values (developed through chapters 3 and 4). This contribution constitutes the second and the fourth objectives of the thesis.

- A set of three formulae (3.1-3.3) for the approximate assessment of the long-term economic impact of minimum flows and maximum ramping rates on a peak hydropower plant. This contribution constitutes the third objective of the thesis.

- A revision of the state of the art of the hydro scheduling models, including past and present commercial tools, capable of dealing jointly with minimum flows and maximum ramping rates (developed through sec. 2.5). The absence, so far, of published contributions concerning the achievements of the first three objectives of the thesis (as discussed in sec. 1.3) was confirmed in that revision.

- Four different methodologies for the assessment of the long-term economic impact of minimum flows and maximum ramping rates on a peak hydropower plant: two of them are deterministic (developed through chapter 3) and the others are stochastic (developed through chapter 4). In each pair, a methodology considers one state variable (stored volume), while the other considers two (stored volume and released flow).

- The definition, determination and application of the flow value (developed through chapter 4). 


\subsection{Main conclusions}

The main conclusions of the thesis are the following:

- The long-term revenue and the water and flow values of a peak hydropower plant are very sensitive to the presence of minimum flows and maximum ramping rates, as well as to their magnitudes.

- The smaller the annual rainfall, the larger the long-term economic impact (in relative terms) caused by minimum flows and maximum ramping rates on hydropeaking.

- From the following two parameters, it is possible to assess the long-term economic impact of minimum flows and maximum ramping rates on hydropeaking: maximum plant flow divided by average river flow and minimum plant flow divided by average river flow.

- The presence of minimum flows in hydropeaking increases the spillage volume and the water value, whereas decreases the generated energy, the number of start-ups and shutdowns of the hydro units, the plant capability for price tracking and the revenue.

- The presence of maximum ramping rates in hydropeaking increases the number of plant operating hours, the spillage volume, and, in the wettest weeks, the flow value, whereas decreases the number of start-ups and shut-downs of the hydro units, the plant capability for price tracking, the revenue, the water value, and, in the driest weeks, the flow value.

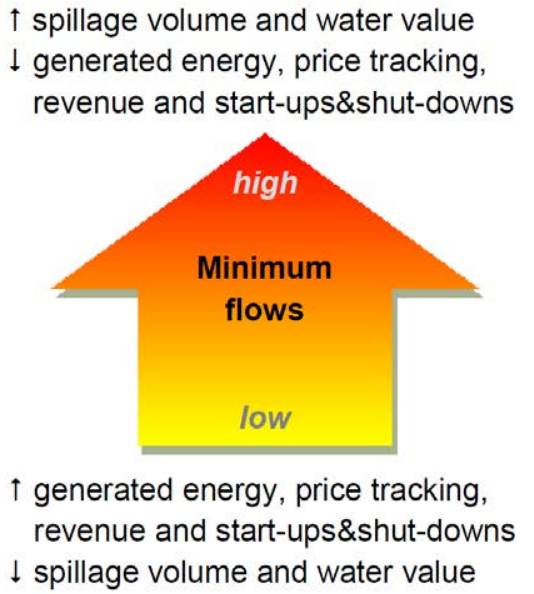

$\uparrow$ spillage volume and water value generated energy, price tracking revenue and start-ups\&shut-downs spillage volume and water value

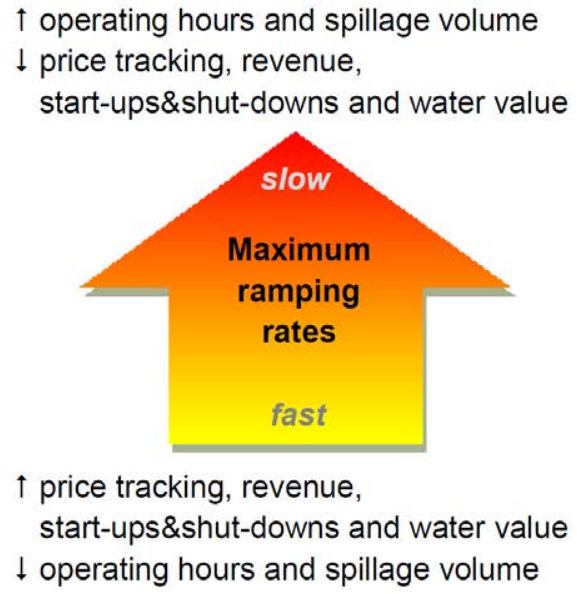

Figure 5.1.: Long-term effects of minimum flows and maximum ramping rates on hydropeaking.

- The consideration of minimum flows for the determination of the water value of a test hydropower plant subject to this constraint provided better economical results than the classical approach, which neglects the influence of minimum flows in the long-term scheduling. 
- The consideration of maximum ramping rates for the determination of the water value of a test hydropower plant subject to this constraint provided worse economical results than the classical approach, which neglects the influence of maximum ramping rates in the long-term scheduling.

\subsection{Future research directions}

Given the logical limitations in the scope of a doctoral thesis and from the obtained results, a trio of main research directions emerge:

- To analyse the convenience of applying the developed methodology within another electricity markets, such as balancing ones. This is an ongoing research line.

- To extend the same objectives pursued in this thesis to entire river basins or pumpedstorage plants.

- To delve into the idea of the flow value and its applicability by testing different numerical realisations of this concept on several peak hydropower plants. 



\section{A. EQUATIONS OF THE OPTIMISATION MODELS}

Fourteen optimisation models were developed along this thesis in order to accomplish its objectives (as discussed in sec.1.3). These models are mainly based on LP (as discussed in sec.2.2.1), DP (as discussed in sec. 2.2.2) or a hybrid combination of both optimisation techniques.

This appendix contains the equations used in the above-mentioned models.

\section{A.1. Equations of the dynamic programming algorithms}

\section{A.1.1. Recursive equations}

- One-dimensional backward deterministic discrete DP.

$$
\begin{array}{r}
Z_{k}^{i v}=\max \left(z_{k}^{i v, f v}+Z_{k+1}^{f v}\right) \\
\forall\{i v, f v\} \in \mathbb{V}_{k} \wedge \forall k \in \mathbb{K}
\end{array}
$$

- One-dimensional stochastic discrete DP.

$$
\begin{array}{r}
\bar{Z}_{k}^{i v, a, b}=\max \left\{z_{k}^{i v, a, b, V_{k}^{f i n}}+\sum_{x}\left[\theta_{k}^{a, x} \cdot \sum_{y}\left(\theta_{k}^{b, y} \cdot \bar{Z}_{k+1}^{V_{k}^{f i n}, x, y}\right)\right]\right\} ; \\
\forall\{a, b, i v, x, y\} \in \mathbb{V W \mathbb { P }}_{k} \wedge \forall k \in \mathbb{K}
\end{array}
$$

- Two-dimensional backward deterministic discrete DP.

$$
\begin{array}{r}
Z_{k}^{i v, i f}=\max \left(z_{k}^{i v, i f, f v, f f}+Z_{k+1}^{f v, f f}\right) ; \\
\forall\{i v, i f, f v, f f\} \in \mathbb{V F}_{k} \wedge \forall k \in \mathbb{K} \wedge \rho>0
\end{array}
$$

- Two-dimensional stochastic discrete DP.

$$
\begin{array}{r}
\bar{Z}_{k}^{i v, i f, a, b}=\max \left\{z_{k}^{i v, i f, a, b, V_{k}^{f i n}, F_{k}^{f i n}}+\sum_{x}\left[\theta_{k}^{a, x} \cdot \sum_{y}\left(\theta_{k}^{b, y} \cdot \bar{Z}_{k+1}^{V_{k}^{f i n}, F_{k}^{f i n}, x, y}\right)\right]\right\} ; \\
\forall\{a, b, i v, i f, x, y\} \in \mathbb{V} \mathbb{F} \mathbb{W P}_{k} \wedge \forall k \in \mathbb{K}
\end{array}
$$




\section{A.1.2. State transition equations}

- Mass balance.

$$
\begin{array}{r}
V_{k+1}^{f v}=V_{k}^{i v}+K_{c 1} \cdot \sum_{t \in \mathbb{T}} w_{t}-V_{k}^{r e l, i v, f v} ; \\
\forall\{i v, f v\} \in \mathbb{V}_{k} \wedge \forall k \in \mathbb{K}
\end{array}
$$

- Mass balance considering evaporation.

$$
\begin{array}{r}
V_{k+1}^{f v}=V_{k}^{i v}+K_{c 1} \cdot \sum_{t \in \mathbb{T}}\left(w_{t}-e_{t}\right)-V_{k}^{r e l, i v, f v} ; \\
\forall\{i v, f v\} \in \mathbb{V}_{k} \wedge \forall k \in \mathbb{K}
\end{array}
$$

- Operational inertia considering the bottom outlets.

$$
\begin{array}{r}
F_{k+1}^{f f}=F_{k}^{i f}+\sum_{t \in \mathbb{T}}\left[\left(q_{t+1}+q_{t+1}^{b o}\right)-\left(q_{t}+q_{t}^{b o}\right)\right] ; \\
\forall\{i f, f f\} \in \mathbb{V F}_{k} \wedge \forall k \in \mathbb{K} \wedge \rho>0
\end{array}
$$

- Operational inertia considering the bottom outlets and the spillways.

$$
\begin{array}{r}
F_{k+1}^{f f}=F_{k}^{i f}+\sum_{t \in \mathbb{T}}\left[\left(q_{t+1}+q_{t+1}^{b o}+q_{t+1}^{s p}\right)-\left(q_{t}+q_{t}^{b o}+q_{t}^{s p}\right)\right] ; \\
\forall\{i f, f f\} \in \mathbb{V F}_{k} \wedge \forall k \in \mathbb{K}
\end{array}
$$

\section{A.1.3. Revenue estimation equations}

- Proportional estimation of the hydropower plant revenue corresponding to the decision to go from $V_{k}^{i v}$ to $V_{k+1}^{f v}$.

$$
\begin{array}{r}
z_{k}^{i v, f v} \propto P_{k} \cdot H_{k}^{i v, f v} \cdot V_{k}^{t u r, i v, f v} ; \\
\forall\{i v, f v\} \in \mathbb{V}_{k} \wedge \forall k \in \mathbb{K}
\end{array}
$$

- Approximated estimation of the water volume released through the hydro units corresponding to the decision to go from $V_{k}^{i v}$ to $V_{k+1}^{f v}$.

$V_{k}^{t u r, i v, f v}=\left\{\begin{array}{l}K_{c 1} \cdot Q^{\max } \cdot t^{\max } \\ V_{k}^{r e l, i v, f v} ; \\ \frac{Q^{\min } \cdot\left(V_{k}^{r e l, i v, f v}-K_{c 1} \cdot \varphi_{k} \cdot t^{\max }\right)}{Q^{\min }-\varphi_{k}} \\ 0\end{array}\right.$

$$
\begin{aligned}
& V_{k}^{r e l, i v, f v} \geq K_{c 1} \cdot Q^{\text {max }} \cdot t^{\text {max }} \\
& K_{c 1} \cdot Q^{\text {min }} \cdot t^{\text {max }} \leq V_{k}^{r e l, i v, f v}<K_{c 1} \cdot Q^{\text {max }} \cdot t^{\text {max }} \\
& \varphi_{k}<Q^{\text {min }} \wedge V_{k}^{r e l, i v, f v}<K_{c 1} \cdot Q^{\text {min }} \cdot t^{\text {max }} \\
& \varphi_{k} \geq Q^{\text {min }} \wedge V_{k}^{r e l, i v, f v}<K_{c 1} \cdot Q^{\text {min }} \cdot t^{\text {max }} \\
& \quad \forall\{i v, f v\} \in \mathbb{V}_{k} \wedge \forall k \in \mathbb{K}
\end{aligned}
$$


- Approximated estimation of the number of operating hours corresponding to the decision to go from $V_{k}^{i v}$ to $V_{k+1}^{f v}$.

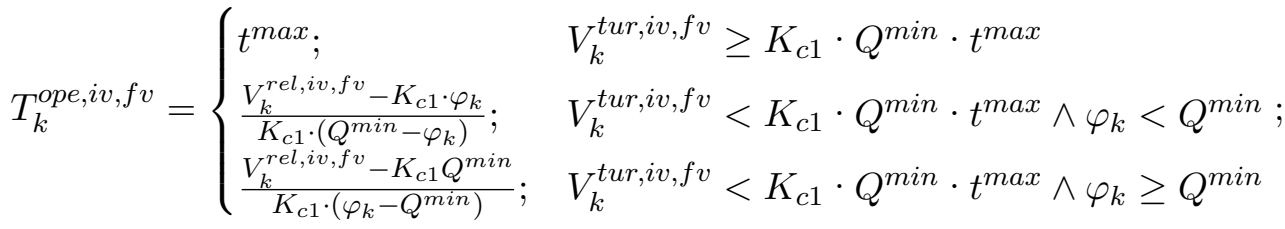

$$
\begin{aligned}
& \forall\{i v, f v\} \in \mathbb{V}_{k} \wedge \forall k \in \mathbb{K}
\end{aligned}
$$

- Estimation of the hydropower plant revenue corresponding to the decision to go from $V_{k}^{i v}$ to $V_{k+1}^{f v}$.

$$
\begin{array}{r}
z_{k}^{i v, f v} \cong P_{k} \cdot T_{k}^{o p e, i v, f v} \cdot G_{k}^{i v, f v} ; \\
\forall\{i v, f v\} \in \mathbb{V}_{k} \wedge \forall k \in \mathbb{K}
\end{array}
$$

\section{A.1.4. Convergence criteria}

- For incremental DP [Nandalal and Bogardi (2007)].

$$
\frac{\left.Z)^{n}-Z\right)^{n-1}}{Z)^{n-1}} \leq 0.1 \%
$$

- For one-dimensional stochastic discrete DP.

$$
\begin{aligned}
& \frac{\left.\left.\mid \bar{Z}_{k}^{i v, a, b}\right)^{n}-\bar{Z}_{k}^{i v, a, b}\right)^{n-1} \mid}{\left.\bar{Z}_{k}^{i v, a, b}\right)^{n-1}} \leq 1 \% ; \\
& \forall\{a, b, i v\} \in \mathbb{V} \mathbb{W P}_{k} \wedge \forall k \in \mathbb{K}
\end{aligned}
$$

- For two-dimensional stochastic discrete DP.

$$
\begin{aligned}
& \frac{\left.\left.\mid \bar{Z}_{k}^{i v, i f, a, b}\right)^{n}-\bar{Z}_{k}^{i v, i f, a, b}\right)^{n-1} \mid}{\left.\bar{Z}_{k}^{i v, i f, a, b}\right)^{n-1}} \leq 3 \% ; \\
& \forall\{a, b, i v, i f\} \in \mathbb{V} \mathbb{F} \mathbb{W P}_{k} \wedge \forall k \in \mathbb{K}
\end{aligned}
$$

\section{A.2. Equations of the linear programming algorithms}

\section{A.2.1. Objective functions}

- Objective function considering the wear and tear costs and the start-up and shut-down costs of the hydro units.

$$
\begin{array}{r}
\max \left\{\sum_{t \in \mathbb{T}}\left(p_{t} \cdot g_{t}\right)-\sum_{t=2}^{t^{\max }}\left[\alpha \cdot\left(g_{t}^{\text {dec }}+g_{t}^{i n c}\right)+\beta \cdot \sum_{u \in \mathbb{U}}\left(s d_{t}^{u}+s u_{t}^{u}\right)\right]\right\} ; \\
t^{\max }=168 \mathrm{~h}
\end{array}
$$


- Objective function for the relaxed final V constraints (Eqs. A.42-A.43) considering the wear and tear costs and the start-up and shut-down costs of the hydro units.

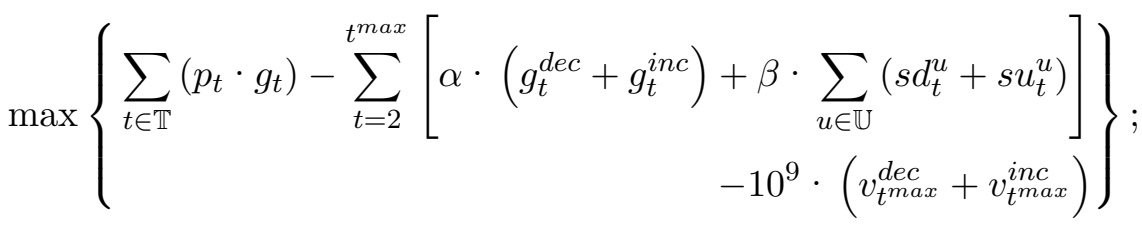

$$
\begin{aligned}
& t^{\max }>168 h \wedge \rho=0 h
\end{aligned}
$$

- Objective function for the relaxed final V and F constraints (Eqs. A.42-A.45) considering the wear and tear costs and the start-up and shut-down costs of the hydro units.

$$
\begin{aligned}
& \max \left\{\sum_{t \in \mathbb{T}}\left(p_{t} \cdot g_{t}\right)-\sum_{t=2}^{t^{\max }}\left[\begin{array}{r}
\left.\alpha \cdot\left(g_{t}^{d e c}+g_{t}^{i n c}\right)+\beta \cdot \sum_{u \in \mathbb{U}}\left(s d_{t}^{u}+s u_{t}^{u}\right)\right] \\
-10^{9} \cdot\left(v_{t^{\text {max }}}^{\text {dec }}+v_{t^{\max }}^{\text {inc }}+f_{t^{\max }}^{\text {dec }}+f_{t^{\text {max }}}^{i n c}\right)
\end{array}\right\} ;\right. \\
& t^{\max }>168 h \wedge \rho>0 h
\end{aligned}
$$

- Objective function considering the wear and tear costs and the start-up and shut-down costs of the hydro units, an artificial cost of the use of the bottom outlets and the spillways, and the expected value of the water at the end of the subproblem.

$$
\max \left\{\begin{array}{r}
\sum_{t \in \mathbb{T}}\left(p_{t} \cdot g_{t}\right)-\sum_{t=2}^{t^{\max }}\left[\alpha \cdot\left(g_{t}^{\text {dec }}+g_{t}^{i n c}\right)+\beta \cdot \sum_{u \in \mathbb{U}}\left(s d_{t}^{u}+s u_{t}^{u}\right)\right. \\
\left.-\gamma \cdot\left(q_{t}^{\text {bospdec }}+q_{t}^{b o s p^{i n c}}\right)\right]+\bar{Z}_{k+1}^{V_{k}^{f i n}}\left({ }^{a, b}\right. \\
\forall\{a, b\} \in \mathbb{V W \mathbb { P }}_{k} \wedge \forall k \in \mathbb{K} \\
\forall ;
\end{array}\right.
$$

- Objective function considering the wear and tear costs and the start-up and shut-down costs of the hydro units, an artificial cost of the use of the bottom outlets and the spillways, and the expected value of the water and the flow at the end of the subproblem.

$$
\max \left\{\begin{array}{r}
\sum_{t \in \mathbb{T}}\left(p_{t} \cdot g_{t}\right)-\sum_{t=2}^{t^{m a x}}\left[\alpha \cdot\left(g_{t}^{d e c}+g_{t}^{i n c}\right)+\beta \cdot \sum_{u \in \mathbb{U}}\left(s d_{t}^{u}+s u_{t}^{u}\right)\right. \\
\left.-\gamma \cdot\left(q_{t}^{\text {bospdec }}+q_{t}^{b o s p^{i n c}}\right)\right]+\bar{Z}_{k+1}^{V_{k}^{f i n}, F_{k}^{f i n}}\left({ }^{a, b}\right. \\
\forall\{a, b\} \in \mathbb{V} \mathbb{W P}_{k} \wedge \forall k \in \mathbb{K} \\
\forall ;
\end{array}\right.
$$

Both $\alpha$ and $\beta$ were determined from the information contained in EPRI (2001) and Nilsson and Sjelvgren (1997a), respectively. $\gamma$ is a small and artificial cost $\left(0.01 € / \mathrm{m}^{3} / \mathrm{s}\right.$ in the study 4 and $10 € / \mathrm{m}^{3} / \mathrm{s}$ in the studies 5 and 6 ; all of them in chapter 4 ) introduced with the aim 
of obtaining a more realistic use of the bottom outlets and spillways. $\gamma$ was neglected during the recalculations of the power outputs and the resulting revenue from the decisions obtained in the LP optimisations with the real generation characteristic of the considered plant.

\section{A.2.2. Constraints}

- $\varphi$ considering the hydro units and the bottom outlets.

As $\varphi$ must be present in all natural flows of the river [Sanguedo Baptista and De Aquino Galeano Massera Da Hora (2013)], it was imposed to be lower than or equal to the current water inflow into the reservoir.

$$
\begin{array}{r}
q_{t}+q_{t}^{b o} \geq \min \left(\varphi_{k}, w_{t}\right) ; \\
\forall t \in \mathbb{T} \wedge \varphi>0 \%
\end{array}
$$

- $\varphi$ considering the hydro units, the bottom outlets and the spillways.

$$
\begin{array}{r}
q_{t}+q_{t}^{b o}+q_{t}^{s p} \geq \min \left(\varphi_{k}, w_{t}\right) ; \\
\forall t \in \mathbb{T} \wedge \varphi>0 \%
\end{array}
$$

- $\rho$ considering the hydro units and the bottom outlets.

The effect of $\rho$ was introduced as in Guan et al. (1999):

$$
\begin{array}{r}
q_{t+1}+q_{t+1}^{b o}-q_{t}-q_{t}^{b o} \leq \rho_{k}^{u p} ; \\
\forall k \in \mathbb{K} \wedge \forall t \in \mathbb{T} \mid t<t^{\text {max }} \wedge \rho>0 h \\
q_{t+1}+q_{t+1}^{b o}-q_{t}-q_{t}^{b o} \geq-\rho_{k}^{\text {down }} ; \\
\forall k \in \mathbb{K} \wedge \forall t \in \mathbb{T} \mid t<t^{\text {max }} \wedge \rho>0 h
\end{array}
$$

- $\rho$ considering the hydro units, the bottom outlets and the spillways.

$$
\begin{gathered}
q_{t+1}+q_{t+1}^{b o}+q_{t+1}^{s p}-q_{t}-q_{t}^{b o}-q_{t}^{s p} \leq \rho_{k}^{u p} ; \\
\forall k \in \mathbb{K} \wedge \forall t \in \mathbb{T} \mid t<t^{\text {max }} \wedge \rho>0 h \\
q_{t+1}+q_{t+1}^{b o}+q_{t+1}^{s p}-q_{t}-q_{t}^{b o}-q_{t}^{s p} \geq-\rho_{k}^{\text {down }} ; \\
\forall k \in \mathbb{K} \wedge \forall t \in \mathbb{T} \mid t<t^{\text {max }} \wedge \rho>0 h
\end{gathered}
$$

- Mass balance considering the bottom outlets.

$$
\begin{array}{r}
v_{t}=v_{t-1}+K_{c 1} \cdot\left(w_{t}-q_{t}-q_{t}^{b o}\right) ; \\
\forall t \in \mathbb{T}
\end{array}
$$


- Mass balance considering the bottom outlets, the spillways and evaporation.

$$
\begin{array}{r}
v_{t}=v_{t-1}+K_{c 1} \cdot\left(w_{t}-q_{t}-q_{t}^{b o}-q_{t}^{s p}-e_{t}\right) ; \\
\forall t \in \mathbb{T}
\end{array}
$$

- Evaporation losses.

$$
\begin{aligned}
e_{t}=K_{c 2} \cdot E_{k} \cdot & \left(K_{e 1} \cdot v_{t}+K_{e 2}\right) ; \\
& \forall k \in \mathbb{K} \wedge \forall t \in \mathbb{T}
\end{aligned}
$$

- Initial and final V of the subproblems within deterministic DP.

$$
\begin{aligned}
& v_{0}= \begin{cases}V^{\#} ; & k=1 \\
V_{k}^{i v} ; & \forall i v \in \mathbb{V}_{k} \wedge \forall k \in \mathbb{K} \mid k>1\end{cases} \\
& v_{t^{\text {max }}}= \begin{cases}V^{\#} ; & k=k^{\max } \\
V_{k}^{f v} ; & \forall f v \in \mathbb{V}_{k} \wedge \forall k \in \mathbb{K} \mid k<k^{\max } \wedge t^{\max }=168 h\end{cases}
\end{aligned}
$$

- Initial V of the subproblems within stochastic DP.

$$
\begin{array}{r}
v_{0}=V_{k}^{i v} ; \\
\forall i v \in \mathbb{V}_{k} \wedge \forall k \in \mathbb{K}
\end{array}
$$

- Initial and final $\mathrm{F}$ of the subproblems defined by refinement (illustrated in Fig. 3.2).

$$
\begin{gathered}
q_{1}=\frac{q_{t^{\text {max }}, k-1}^{o s}+q_{1, k}^{o s}}{2} ; \\
\forall k \in \mathbb{K} \mid k>1 \wedge \rho>0 h \\
q_{t^{\max }}=\frac{q_{t^{\text {max }}, k}^{o s}+q_{1, k+1}^{o s}}{2} ; \\
\forall k \in \mathbb{K} \mid k<k^{\max } \wedge \rho>0 h
\end{gathered}
$$

- Initial and final $\mathrm{F}$ of the subproblems considering the hydro units within deterministic $\mathrm{DP}$ with $\mathrm{F}$ as state variable.

$$
\begin{array}{r}
q_{1}=F_{k}^{i f} ; \\
\forall i f \in \mathbb{V F}_{k} \wedge \forall k \in \mathbb{K} \mid k>1 \wedge \rho>0 h
\end{array}
$$




$$
\begin{array}{r}
q_{t^{\max }}=F_{k+1}^{f f} ; \\
\forall f f \in \mathbb{V F}_{k} \wedge \forall k \in \mathbb{K} \mid k<k^{\max } \wedge \rho>0 h
\end{array}
$$

- Initial and final $\mathrm{F}$ of the subproblems considering the hydro units and the bottom outlets within deterministic DP with $\mathrm{F}$ as state variable.

$$
\begin{gathered}
q_{0}+q_{0}^{b o} \leq F_{k}^{i f}+\rho_{k}^{u p} ; \\
\forall i f \in \mathbb{V F}_{k} \wedge \forall k \in \mathbb{K} \mid k>1 \wedge \rho>0 h \\
q_{0}+q_{0}^{b o} \geq F_{k}^{i f}-\rho_{k}^{\text {down }} ; \\
\forall i f \in \mathbb{V F}_{k} \wedge \forall k \in \mathbb{K} \mid k>1 \wedge \rho>0 h \\
\forall f f \in \mathbb{V F}_{k} \wedge \forall k \in \mathbb{K} \mid k<k^{\text {max }} \wedge t^{\text {max }}=168 h \wedge \rho>0 h
\end{gathered}
$$

- Initial $\mathrm{F}$ of the subproblems considering the hydro units, the bottom outlets and the spillways.

$$
\begin{gathered}
q_{0}+q_{0}^{b o}+q_{0}^{s p} \leq F_{k}^{i f}+\rho_{k}^{u p} ; \\
\forall i f \in \mathbb{V F}_{k} \wedge \forall k \in \mathbb{K} \mid k>1 \\
q_{0}+q_{0}^{b o}+q_{0}^{s p} \geq F_{k}^{i f}-\rho_{k}^{\text {down }} ; \\
\forall i f \in \mathbb{V F}_{k} \wedge \forall k \in \mathbb{K} \mid k>1
\end{gathered}
$$

- Relaxation of the state boundary conditions at the end of the subproblems within deterministic DP.

$$
\begin{gathered}
v_{t^{\max }}^{\text {dec }} \geq V_{k+1}^{f v}-v_{t^{\max }} ; \\
\forall f v \in \mathbb{V}_{k} \wedge \forall k \in \mathbb{K} \wedge t^{\text {max }}>168 h \\
\forall f v \in \mathbb{V}_{k} \wedge \forall k \in \mathbb{K} \wedge t^{\max }>168 h \\
v_{t^{\max }}^{\text {inc }} \geq v_{t^{\max }}-V_{k+1}^{f v} ; \\
\forall f f \in \mathbb{V F}_{k} \wedge \forall k \in \mathbb{K} \wedge t^{\max }>168 h \wedge \rho>0 h \\
f_{t^{\text {max }}}^{\text {dec }} \geq F_{k+1}^{f f}-q_{t^{\max }}-q_{t^{\text {max }}}^{\text {bo }} ;
\end{gathered}
$$




$$
\begin{array}{r}
f_{t^{\max }}^{i n c} \geq q_{t^{\max }}+q_{t^{\max }}^{b o}-F_{k+1}^{f f} ; \\
\forall f f \in \mathbb{V F}_{k} \wedge \forall k \in \mathbb{K} \wedge t^{\max }>168 h \wedge \rho>0 h
\end{array}
$$

- Maximum legal water content of the reservoir.

Although River Basin District Authorities usually demand the strict fulfilment of the maximum legal water content of the reservoirs (considered in chapter 3 ):

$$
\begin{array}{r}
v_{t} \leq V_{k}^{\text {maxleg }} ; \\
\forall k \in \mathbb{K} \wedge \forall t \in \mathbb{T}
\end{array}
$$

its application was limited to the last hour of each week in chapter 4 because of numerous problems of infeasibility caused by the considered stochasticity of the water inflows and its subsequent floods:

$$
\begin{array}{r}
v_{t^{\max }} \leq V_{k}^{\text {maxleg }} ; \\
\forall k \in \mathbb{K}
\end{array}
$$

- Maximum technical water content of the reservoir.

$$
\begin{array}{r}
v_{t} \leq V^{\text {maxtec }} ; \\
\forall t \in \mathbb{T}
\end{array}
$$

- Minimum technical water content of the reservoir below which it is not convenient or possible to generate power.

$$
\begin{array}{r}
v_{t} \geq V^{\text {mintec }} ; \\
\forall t \in \mathbb{T}
\end{array}
$$

- Minimum water content of the reservoir below which it is not possible to release water.

$$
\begin{array}{r}
v_{t} \geq V^{\text {dead }} ; \\
\forall t \in \mathbb{T}
\end{array}
$$

- Flow released through the hydro units.

$$
\begin{array}{r}
q_{t}=o l_{t}^{1} \cdot q^{1}+\sum_{s \in \mathbb{S}} q_{t}^{s} ; \\
\forall t \in \mathbb{T}
\end{array}
$$


- Plant generation characteristic model of piecewise linear curves.

The plant generation characteristics used in the thesis were modelled by series of one or more performance piecewise linear curves, each curve corresponding to a different water content of the considered reservoir (as in Conejo et al. (2002)). This model was selected for its balance between accuracy and simplicity (as discussed in sec. 2.4.1).

The number of performance curves used in each subproblem was selected according to the degree of accuracy pursued in each study and to the maximum range of head available during the subproblem:

$$
\begin{aligned}
& c^{\max }=\min \left\{\overline{c^{\max }},\left\lfloor\frac{h_{k}^{\max f, i v, f v}-h_{k}^{\operatorname{minf}, i v, f v}}{H^{\%} \cdot\left(H_{k}^{\max }-H_{k}^{\min }\right)}\right\rfloor+1\right\} ; \\
& \forall\{i v, f v\} \in \mathbb{V}_{k} \wedge \forall k \in \mathbb{K}
\end{aligned}
$$

$h_{k}^{\max f, i v, f v}\left(h_{k}^{\operatorname{minf}, i v, f v}\right)$ was calculated assuming that $\varphi_{k}\left(Q^{\max }\right)$ was released during the first $n$ hours of the subproblem and that $Q^{\max }\left(\varphi_{k}\right)$ was released during the last $\left(t^{\max }-n\right)$ hours of the subproblem; $n$ was obtained in a time-volume diagram as the intersection of two lines beginning at the initial and final reservoir volumes of the stage. $\overline{c^{\max }}$ and $H^{\%}$ was selected depending on the pursued accuracy in each study.

Once $c^{\max }$ was determined, the generated power was defined by:

$$
\begin{aligned}
& g_{t}=o l_{t}^{1} \cdot g^{\min }+\sum_{s \in \mathbb{S}}\left(q_{t}^{s} \cdot r^{s}\right) \\
& \forall t \in \mathbb{T} \wedge c^{\max }=1 \\
& g_{t} \leq o l_{t}^{1} \cdot g^{\min , c}+\sum_{s \in \mathbb{S}}\left(q_{t}^{s} \cdot r^{s, c}\right)+\left(c-1-\sum_{c=1}^{c-1} p c_{t}^{c}+\sum_{c=c}^{c^{\max }-1} p c_{t}^{c}\right) \cdot g^{\max } \\
& \forall t \in \mathbb{T} \wedge \forall c \in \mathbb{C}_{k}^{i v, f v} \mid \underset{s}{c}>1 \wedge c^{\max }>1 \wedge \forall\{i v, f v\} \in \mathbb{V}_{k} \wedge \forall k \in \mathbb{K} \\
& g_{t} \geq o l_{t}^{1} \cdot g^{\min , c}+\sum_{s \in \mathbb{S}}\left(q_{t}^{s} \cdot r^{s, c}\right)-\left(c-1-\sum_{c=1}^{c-1} p c_{t}^{c}+\sum_{c=c}^{c_{s}^{\max }-1} p c_{t}^{c}\right) \cdot g^{\max } ; \\
& \forall t \in \mathbb{T} \wedge \forall c \in \mathbb{C}_{k}^{i v, f v} \mid \underset{s}{c}>1 \wedge c^{\max }>1 \wedge \forall\{i v, f v\} \in \mathbb{V}_{k} \wedge \forall k \in \mathbb{K} \\
& p c_{t}^{c} \geq p c_{t}^{c+1} \\
& \forall t \in \mathbb{T} \wedge \forall c \in \mathbb{C}_{k}^{i v, f v}\left|c<c^{\max }-1\right| c^{\max }>1 \wedge \forall\{i v, f v\} \in \mathbb{V}_{k} \wedge \forall k \in \mathbb{K}
\end{aligned}
$$




$$
\begin{aligned}
& v_{t} \geq \sum_{c=1}^{c^{\max }-2}\left[\left(p c_{t}^{c}-p c_{t}^{c+1}\right) \cdot v^{c}\right]+p c_{t}^{c^{\max }-1} \cdot v^{c^{\max }-1} ; \\
& \forall t \in \mathbb{T} \wedge c^{\max }>1 \\
& v_{t} \leq p c_{t}^{c^{\max }-1} \cdot v_{k}^{\max f, i v, f v}+\left(1-p c_{t}^{1}\right) \cdot v^{1}+\sum_{c=1}^{c^{\max }-2}\left[\left(p c_{t}^{c}-p c_{t}^{c+1}\right) \cdot v^{c+1}\right] ; \\
& \forall t \in \mathbb{T} \wedge c^{\max }>1 \wedge \forall\{i v, f v\} \in \mathbb{V}_{k} \wedge \forall k \in \mathbb{K}
\end{aligned}
$$

It must be noted that in the case of several performance curves, the segments that result from joining the suggested break points may have different lengths in each performance curve. In order for the algorithm to perform correctly, the length of each segment must be equal in all curves. For this reason, these lengths were calculated as the means of the lengths of the segments of all (every meter) performance curve comprised within the interval $\left[h_{k}^{\operatorname{minf}, i v, f v}, h_{k}^{\max f, i v, f v}\right]$; the used performance curves were consistently modified.

The shape of the performance curves were defined in two different ways according to the degree of accuracy pursued in each study of the thesis: concave or non-concave. On the one hand, when the concave approach was used, each performance curve was forced to comprise $s^{\max }$ linear segments with break points at the points corresponding to minimum power, local best efficiencies and maximum total flow (as in Habibollahzadeh and Bubenko (1986)) in order to reduce the computational burden [Borghetti et al. (2008)]:

$$
\begin{aligned}
& s^{\max }=u^{\max }+1 \\
& q_{t}^{s} \leq o l_{t}^{1} \cdot q^{\max , s} ; \\
& \forall s \in \mathbb{S} \wedge \forall t \in \mathbb{T}
\end{aligned}
$$

On the other hand, when the non-concave approach was selected, each performance curve showed break points at the same locations as in the concave version plus those corresponding to the plant flows beyond which each hydro unit starts up and some additional ones used to guarantee that the lengths of all segments were lower or equal to the $10 \%$ of the maximum variation of the plant flow:

$$
\begin{aligned}
& q_{t}^{s} \leq\left\{\begin{array}{ll}
o l_{t}^{u} \cdot q^{\max , s} ; & \forall s \in \mathbb{S}\left|s^{u} \leq s \leq s^{u+1} \wedge u \in \mathbb{U}\right| 1 \leq u \leq u^{\text {max }} \\
o l_{t}^{u^{\max }} \cdot q^{\max , s} ; & \forall s \in \mathbb{S} \mid s^{u} \geq s^{u^{\max }}
\end{array} ; \forall t \in \mathbb{T}\right. \\
& q_{t}^{s} \geq o l_{t}^{u} \cdot q^{\text {max }, s} ; \forall s \in \mathbb{S}\left|s^{u-1} \leq s \leq s^{u} \wedge u \in \mathbb{U}\right| u>1 \wedge \forall t \in \mathbb{T}
\end{aligned}
$$


- Inter-hourly variation of the generated power.

$$
\begin{array}{r}
g_{t}^{i n c}-g_{t}^{d e c}=g_{t+1}-g_{t} ; \\
\forall t \in \mathbb{T} \mid t<t^{\text {max }}
\end{array}
$$

- Start-up and shut-down of the hydro units.

The start-ups and shut-downs of the hydro units were introduced according to the formulation proposed in Arroyo and Conejo (2004):

$$
\begin{aligned}
& q_{t} \geq o l_{t}^{1} \cdot q^{1}+o l_{t}^{2} \cdot\left(q^{2}-q^{1}\right)+\sum_{u=3}^{u^{\max }}\left[o l_{t}^{u} \cdot\left(q^{u}-q^{u-1}\right)\right] ; \\
& \forall t \in \mathbb{T} \\
& q_{t} \leq o l_{t}^{1} \cdot q^{2}+\sum_{u=2}^{u^{\max }-1}\left[o l_{t}^{u} \cdot\left(q^{u+1}-q^{u}\right)\right]+o l_{t}^{u^{\max }} \cdot\left(q^{\max }-q^{u^{\max }}\right) ; \\
& s u_{t+1}^{u}-s d_{t+1}^{u}=o l_{t+1}^{u}-o l_{t}^{u} ; \\
& \forall u \in \mathbb{U} \wedge \forall t \in \mathbb{T} \mid t<t^{\text {max }} \\
& \forall d_{t}^{u}+s u_{t}^{u} \leq 1 ; \\
& \forall u \in \mathbb{U} \wedge \forall t \in \mathbb{T} \\
& \forall u \in \mathbb{U} \wedge \forall t \in \mathbb{T} \mid t<t^{\max } \\
& o l_{t}^{u} \geq o l_{t}^{u+1} ;
\end{aligned}
$$

Maximum technical plant flow.

$$
\begin{array}{r}
q_{t} \leq \sum_{d \in \mathbb{D}}\left(Q^{\text {max }, d} \cdot v q_{t}^{d}\right) \\
\forall t \in \mathbb{T} \\
v_{t} \geq \sum_{d \in \mathbb{D}}\left(V Q^{d} \cdot v q_{t}^{d}\right) ; \\
\forall t \in \mathbb{T}
\end{array}
$$




$$
\begin{array}{r}
o l_{t}^{1} \leq v q_{t}^{1} \\
\forall t \in \mathbb{T}
\end{array}
$$

- Maximum flow through the bottom outlets.

For practical reasons, Taintor type gates were assumed to be installed in the bottom outlets of all studied plants, being therefore possible to modify the released flow in a continuous way from 0 to the maximum available value (Morgan et al. (1957)). This latter was considered in two different ways according to the degree of accuracy pursued in each study of the thesis.

In chapter 3, the maximum available flow through the bottom outlets was calculated in each subproblem from the mean of its gross head and it was introduced constant into the formulation:

$$
\begin{array}{r}
q_{t}^{b o} \leq Q^{\text {maxbo }} ; \\
\forall t \in \mathbb{T}
\end{array}
$$

On the other hand, in chapter 4 , this flow was hourly calculated by means of a linear approximation (e.g. illustrated in Fig. B.4) of the real values according to V:

$$
\begin{array}{r}
q_{t}^{b o} \leq K_{b o 1} \cdot v_{t}+K_{b o 2} ; \\
\forall t \in \mathbb{T}
\end{array}
$$

- Maximum flow over the spillways.

$$
\begin{gathered}
q_{t}^{s p} \leq K_{s p} \cdot v_{t}^{a b o s p} ; \\
\forall t \in \mathbb{T} \\
v_{t}=v_{t}^{a b o s p}+v_{t}^{\text {belsp }} ; \\
\forall t \in \mathbb{T} \\
v_{t}^{a b o s p} \leq\left(V^{\text {maxtec }}-V^{s p}\right) \cdot s p_{t} ; \\
\forall t \in \mathbb{T} \\
s p_{t} \leq \frac{v_{t}^{\text {belsp }}}{V^{s p}} ; \\
\forall t \in \mathbb{T}
\end{gathered}
$$




$$
\begin{array}{r}
v_{t}^{b e l s p} \leq V^{s p} \\
\forall t \in \mathbb{T}
\end{array}
$$

- Inter-hourly variation of the total flow released through the bottom outlets and/or over the spillways.

$$
\begin{array}{r}
q_{t}^{\text {bosp } p^{i n c}}-q_{t}^{\text {bospdec }}=q_{t+1}^{b o}+q_{t+1}^{s p}-q_{t}^{b o}-q_{t}^{s p} ; \\
\forall t \in \mathbb{T} \mid t<t^{\text {max }}
\end{array}
$$

- Future expected revenue at the end of the subproblem considering the water value.

$$
\begin{gathered}
\bar{Z}_{k+1}^{V_{k}^{f i n}}\left(_{j \in \mathbb{J}}^{a, b}=\sum_{k w v^{j} \cdot W V_{k+1}^{j}\left({ }^{a, b}\right) ;}(a, b\} \in \mathbb{V} \mathbb{W P}_{k} \wedge \forall k \in \mathbb{K}\right. \\
v_{t^{\text {max }}}=V^{\text {dead }}+\sum_{j \in \mathbb{J}} v w v^{j} \\
v w v^{j} \leq V W V^{j} \cdot w v^{j} ; \\
\forall j \in \mathbb{J} \\
v w v^{j} \geq V W V^{j} \cdot w v^{j+1} ; \\
\forall j \in \mathbb{J} \mid j<j^{\text {max }} \\
\forall j \in \mathbb{J} \mid j<j^{\text {max }}
\end{gathered}
$$

- Future expected revenue at the end of the subproblem considering the water and the flow value.

Despite its computational burden, the one-dimensional method (as discussed in sec. 2.4.1) was selected to model the new two-dimensional nature of the expected revenue since its application is restricted to the last hour of each subproblem.

$$
\begin{aligned}
& \bar{Z}_{k}^{V_{i}^{f i n}, F_{k}^{f i n}}\left({ }^{a, b} \leq \sum_{m f=1}^{m f^{m a x}-1} c q^{m f} \cdot \bar{z}^{m v, m f, a, b}+\left(1-b v^{m v}\right) \cdot \bar{z}^{m v^{m a x}, m f^{m a x}, a, b} ;\right. \\
& \forall\{a, b\} \in \mathbb{V} \mathbb{F}_{\mathbb{W}} \wedge \mathbb{P}_{k} \wedge \forall k \in \mathbb{K} \wedge \forall m v \in \mathbb{M}^{V} \mid m v<m v^{\text {max }}
\end{aligned}
$$




$$
\begin{aligned}
& \bar{Z}_{k}^{V_{k}^{f i n}, F_{k}^{f i n}}\left({ }^{a, b} \geq \sum_{m f=1}^{m f^{m a x}-1} c q^{m f} \cdot \bar{z}^{m v, m f, a, b}-\left(1-b v^{m v}\right) \cdot \bar{z}^{m v^{m a x}, m f^{m a x}, a, b} ;\right. \\
& \forall\{a, b\} \in \mathbb{V} \mathbb{F} \mathbb{W P}_{k} \wedge \forall k \in \mathbb{K} \wedge \forall m v \in \mathbb{M}^{V} \mid m v<m v^{\text {max }} \\
& c q^{1} \leq b q^{1}
\end{aligned}
$$

$$
c q^{m f} \leq b q^{m f-1}+b q^{m f}
$$

$$
\forall m f \in \mathbb{M}^{F} \mid 1<m f<m f^{\max }
$$

$$
c q^{m f^{\max }} \leq b q^{m f^{\max }-1}
$$

$\sum_{m f \in \mathbb{M}^{F}} c q^{m f}=1$

$$
\sum_{m f=1}^{m f^{m a x}-1} b q^{m f}=1
$$

$$
q_{t^{\max }}+q_{t^{\max }}^{b o}+q_{t^{\max }}^{s p}=\sum_{m f \in \mathbb{M}^{F}}\left(c q^{m f} \cdot \bar{f}^{m f}\right)
$$

$$
v_{t^{\max }} \leq \sum_{m v=1}^{m v^{\max }-1} b v^{m v} \cdot \bar{v}^{m v+1}
$$

$$
v_{t^{\max }} \geq \sum_{m v=1}^{m v^{\max }-1} b v^{m v} \cdot \bar{v}^{m v}
$$

$$
\sum_{m v=1}^{m v^{m a x}-1} b v^{m v}=1
$$




\section{B. MAIN DATA OF THE CASE STUDIES}

Twelve real peak hydropower plants located in several regions of Spain have studied along this thesis in order to accomplish its objectives (as discussed in sec. 1.3). In addition to the availability of their data, two main criteria have been used to select each of these hydropower plants. First, every hydropower plant must be located at the head of its basin with the aim of ignoring the influence of the rest of the water basin regulation infrastructures, without a significant loss of accuracy as well as of easing the characterisation of the water inflows to its reservoir. Second, every hydropower plant must also show, as far as possible, different characteristics with respect to the other hydropower plants in order to increase the diversity and representativity of the sample.

Most of the considered technical data of the hydropower plants used in the thesis have been provided either by the companies that own and manage them or by the river basin authorities under whose jurisdiction the hydropower plants operate. The most problematic data to gather have been the performance curves; whenever this information was not available, performance curves contained in USBR (1976) have been used, conveniently adjusted to the respective parameters of the hydropower plant. The second data most difficult to obtain have been the head-storage curves of the reservoirs; again, whenever this information was not available, head-storage curves have been estimated by means of second-order polynomial functions [Bayón et al. (2008)], properly fixed at the respective parameters of the reservoir.

Three stochastic variables have been introduced in some or all the models of the thesis: water inflows, energy prices and evaporation rates. Regarding the daily and monthly water inflows, their rainfall probability density functions have been obtained from Pérez González and Sanz Donaire (2001) and their historical series have been taken from the web pages of the Centre for Public Works Studies and Experimentation [CEDEX (2013)] and of the Catalan Water Agency [ACA (2015)]. Relating to the hourly energy prices, its historical series has been taken from the web page of the Iberian Electricity Market Operator [OMIE (2011)]. As regards the weekly evaporation rates, these values have been estimated thanks to the application of the empirical temperature-based formula proposed in Dragoni and Valigi (1994) and of the average monthly temperatures took from the web page of the Spanish Statistical Office [INE (1960)].

This appendix provides a summary of the main data of the above-mentioned hydropower plants by means of several self-explanatory figures and tables. 
Table B.1.: Main design parameters of the plant I used in the studies 1 and 2 .

\begin{tabular}{|c|c|c|}
\hline PARAMETER & VALUE & Unit \\
\hline \hline Maximum plant flow & 279 & $\mathrm{~m}^{3} / \mathrm{s}$ \\
\hline Minimum plant flow & 40 & $\mathrm{~m}^{3} / \mathrm{s}$ \\
\hline Maximum outlet flow & 159 & $\mathrm{~m}^{3} / \mathrm{s}$ \\
\hline Average river flow & 91.2 & $\mathrm{~m}^{3} / \mathrm{s}$ \\
\hline Maximum net head & 132 & $\mathrm{~m}$ \\
\hline Minimum net head & 72 & $\mathrm{~m}$ \\
\hline Maximum power & 316.8 & $\mathrm{MW}$ \\
\hline Minimum power & 22.2 & $\mathrm{MW}$ \\
\hline Hydro units & 3 & $\mathrm{Francis}$ \\
\hline Maximum legal storage capacity (15 ${ }^{\text {th }}$ Oct.-15 $5^{\text {th }}$ Apr.) & 607.6 & $\mathrm{hm}^{3}$ \\
\hline Maximum legal storage capacity (16 th $^{\text {Apr.-14 }}{ }^{\text {th }}$ Oct.) & 644.6 & $\mathrm{hm}^{3}$ \\
\hline Maximum technical storage capacity & 644.6 & $\mathrm{hm}^{3}$ \\
\hline Minimum technical storage capacity & 71.0 & $\mathrm{hm}^{3}$ \\
\hline
\end{tabular}

Table B.2.: Main design parameters of the plant I used in the study 4 and of the plant B used in the studies 5 and 6.

\begin{tabular}{|c|c|c|c|}
\hline \multirow{2}{*}{ Parameter } & \multicolumn{2}{|c|}{ Denomination OF THE Plant } & \multirow{2}{*}{ Unit } \\
\cline { 2 - 3 } & I & B & \\
\hline \hline Maximum plant flow & 279 & 16 & $\mathrm{~m} / \mathrm{s}$ \\
\hline Minimum plant flow & 40 & 4 & $\mathrm{~m} / \mathrm{s}$ \\
\hline Maximum outlet flow & 159 & 29 & $\mathrm{~m}^{3} / \mathrm{s}$ \\
\hline Maximum spillway flow & 2,425 & 360 & $\mathrm{~m}^{3} / \mathrm{s}$ \\
\hline Average river flow & 91.2 & 4.4 & $\mathrm{~m}^{3} / \mathrm{s}$ \\
\hline Maximum net head & 132 & 160 & $\mathrm{~m}$ \\
\hline Minimum net head & 72 & 142 & $\mathrm{~m}$ \\
\hline Maximum power & 312.5 & 21.6 & $\mathrm{MW}$ \\
\hline Minimum power & 22.2 & 4.8 & $\mathrm{MW}$ \\
\hline Hydro units & 3 & 2 & $\mathrm{Francis}$ \\
\hline Maximum legal storage capacity (15 ${ }^{\text {th }}$ Oct.-15 ${ }^{\text {th }}$ Apr.) & 607.6 & 45.0 & $\mathrm{hm}^{3}$ \\
\hline Maximum legal storage capacity (16 ${ }^{\text {th }}$ Apr.-14 $4^{\text {th }}$ Oct.) & 644.6 & & $\mathrm{hm}^{3}$ \\
\hline Maximum technical storage capacity & 654.1 & 46.0 & $\mathrm{hm}^{3}$ \\
\hline Minimum technical storage capacity & 71.0 & 18.8 & $\mathrm{hm}^{3}$ \\
\hline Dead storage capacity & 48.1 & 9.0 & $\mathrm{hm}^{3}$ \\
\hline
\end{tabular}


Table B.3.: Main design parameters of the plants used in the study 3 .

\begin{tabular}{|c|c|c|c|c|c|c|c|c|c|c|c|c|}
\hline & \multicolumn{12}{|c|}{ DENOMINATION OF THE PLANT } \\
\hline PARAMETERS & I & II & III & IV & $\mathrm{V}$ & VI & VII & VIII & IX & $\mathrm{X}$ & $\mathrm{A}$ & $\mathrm{B}$ \\
\hline $\begin{array}{c}\text { Maximum } \\
\text { plant flow } \\
\left(\mathrm{m}^{3} / \mathrm{s}\right)\end{array}$ & 279 & 120 & 63 & 70 & 168 & 14 & 8 & 45 & 8 & 100 & 22 & 16 \\
\hline $\begin{array}{c}\text { Minimum } \\
\text { plant flow } \\
\left(\mathrm{m}^{3} / \mathrm{s}\right)\end{array}$ & 40 & 25 & 8 & 19 & 16 & 3 & 4 & 19 & 2 & 15 & 10 & 4 \\
\hline $\begin{array}{c}\text { Maximum } \\
\text { outlet flow } \\
\left(\mathrm{m}^{3} / \mathrm{s}\right)\end{array}$ & 159 & 357 & 239 & 140 & 63 & 48 & 72 & 79 & 45 & 432 & 18 & 29 \\
\hline $\begin{array}{c}\text { Average } \\
\text { river flow } \\
\left(\mathrm{m}^{3} / \mathrm{s}\right)\end{array}$ & 91.2 & 24.9 & 27.3 & 12.9 & 55.2 & 5.3 & 3.0 & 11.2 & 0.6 & 7.7 & 4.2 & 4.4 \\
\hline $\begin{array}{c}\text { Maximum } \\
\text { net head } \\
(m)\end{array}$ & 132 & 81 & 72 & 84 & 112 & 42 & 120 & 155 & 20 & 91 & 259 & 160 \\
\hline $\begin{array}{l}\text { Minimum } \\
\text { net head } \\
(m)\end{array}$ & 72 & 52 & 42 & 47 & 61 & 24 & 76 & 92 & 11 & 38 & 230 & 142 \\
\hline $\begin{array}{c}\text { Maximum } \\
\text { power } \\
(M W)\end{array}$ & 316.8 & 81.7 & 38.1 & 52.4 & 158.6 & 5.2 & 8.4 & 58.6 & 1.4 & 76.4 & 48.0 & 21.6 \\
\hline $\begin{array}{l}\text { Minimum } \\
\text { power } \\
(M W)\end{array}$ & 22.2 & 10.6 & 2.7 & 7.2 & 7.8 & 0.5 & 2.6 & 14.4 & 0.2 & 4.3 & 18.9 & 4.8 \\
\hline $\begin{array}{c}\text { Rainfall } \\
\text { probability } \\
\text { density } \\
\text { function }\end{array}$ & $\begin{array}{l}\bar{\Xi} \\
\frac{\overrightarrow{0}}{0} \\
\overrightarrow{3}\end{array}$ & $\begin{array}{l}\vec{\Xi} \\
\frac{0}{0} \\
\overrightarrow{0}\end{array}$ & 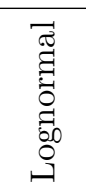 & $\begin{array}{l}\overrightarrow{\widetilde{\Xi}} \\
\overrightarrow{0} \\
0 \\
0.0 \\
0 \\
0\end{array}$ & 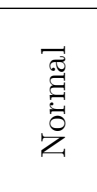 & $\begin{array}{l}\overline{\widetilde{\Xi}} \\
\text { : } \\
0 \\
0 \\
0 \\
0\end{array}$ & 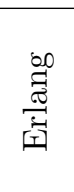 & 苛 & 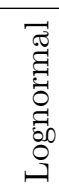 & 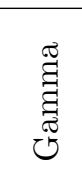 & 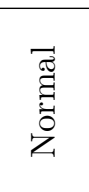 & 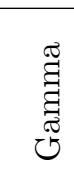 \\
\hline $\begin{array}{l}\text { Number of } \\
\text { hydro units }\end{array}$ & 3 & 2 & 3 & 2 & 4 & 1 & 1 & 1 & 2 & 2 & 1 & 2 \\
\hline $\begin{array}{c}\text { Type of } \\
\text { hydro units }\end{array}$ & 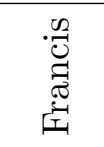 & 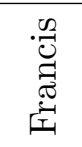 & 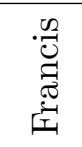 & 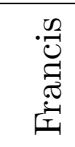 & 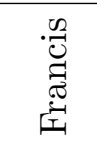 & 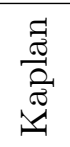 & 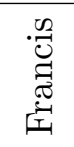 & 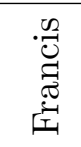 & 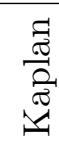 & 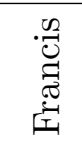 & 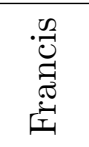 & 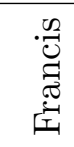 \\
\hline $\begin{array}{c}\text { Useful } \\
\text { water } \\
\text { volume } \\
\left(\mathrm{hm}^{3}\right)\end{array}$ & 574 & 231 & 78 & 141 & 224 & 157 & 62 & 89 & 26 & 885 & 86 & 26 \\
\hline
\end{tabular}




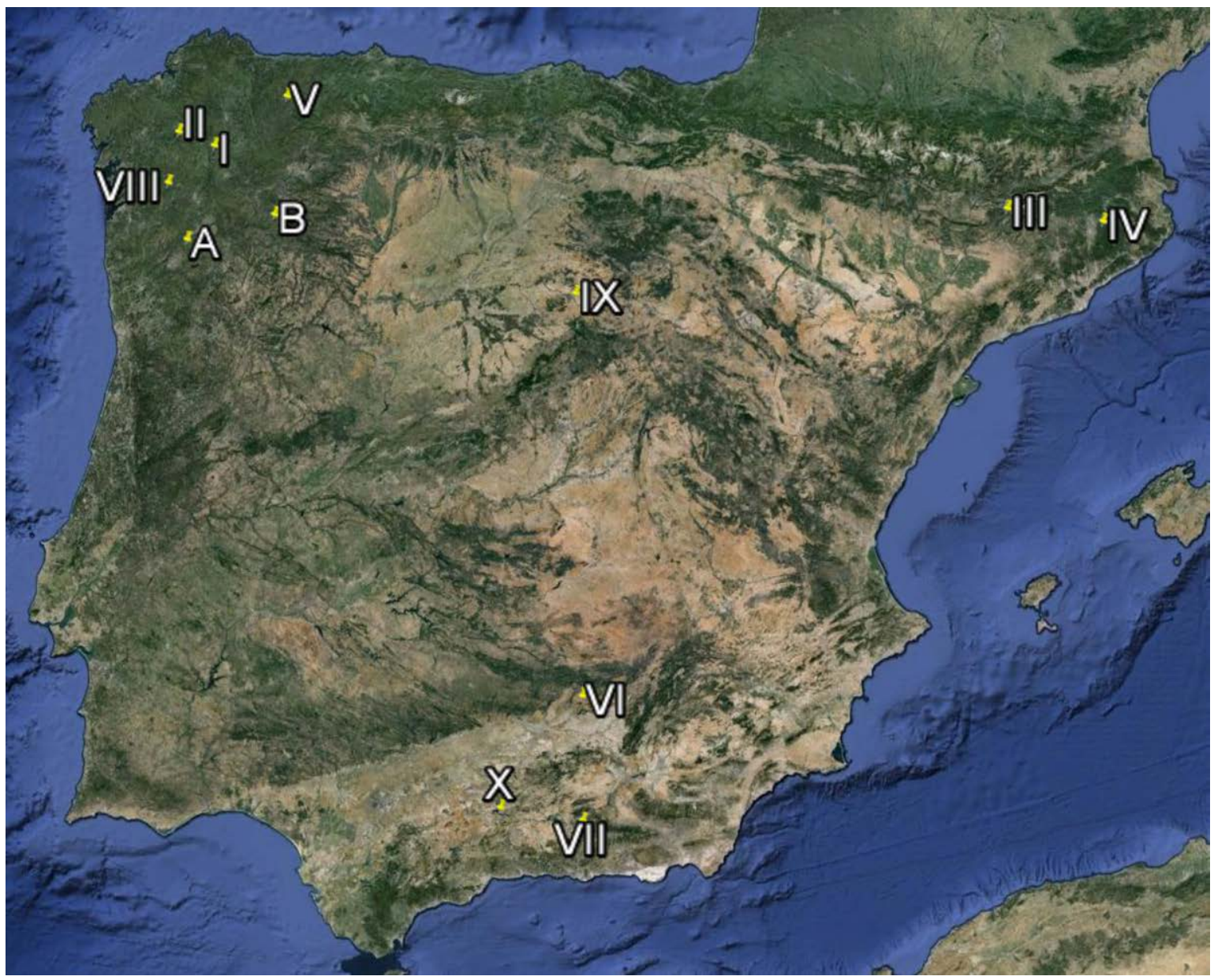

Figure B.1.: Location of the plants used in the thesis (image taken from Google Earth ${ }^{\mathrm{TM}}$ ).
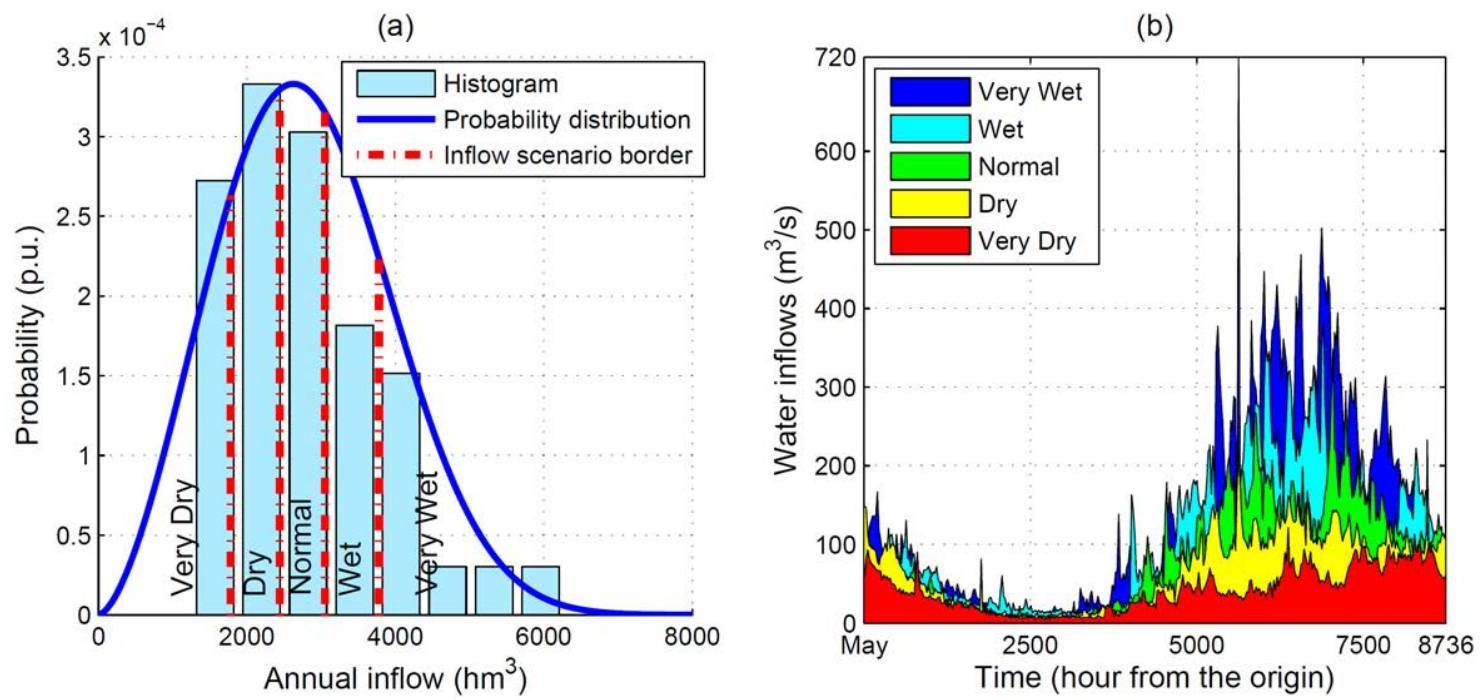

Figure B.2.: Water inflow scenarios of study 1: (a) Histogram and probability distribution; (b) Hourly values for each scenario. 


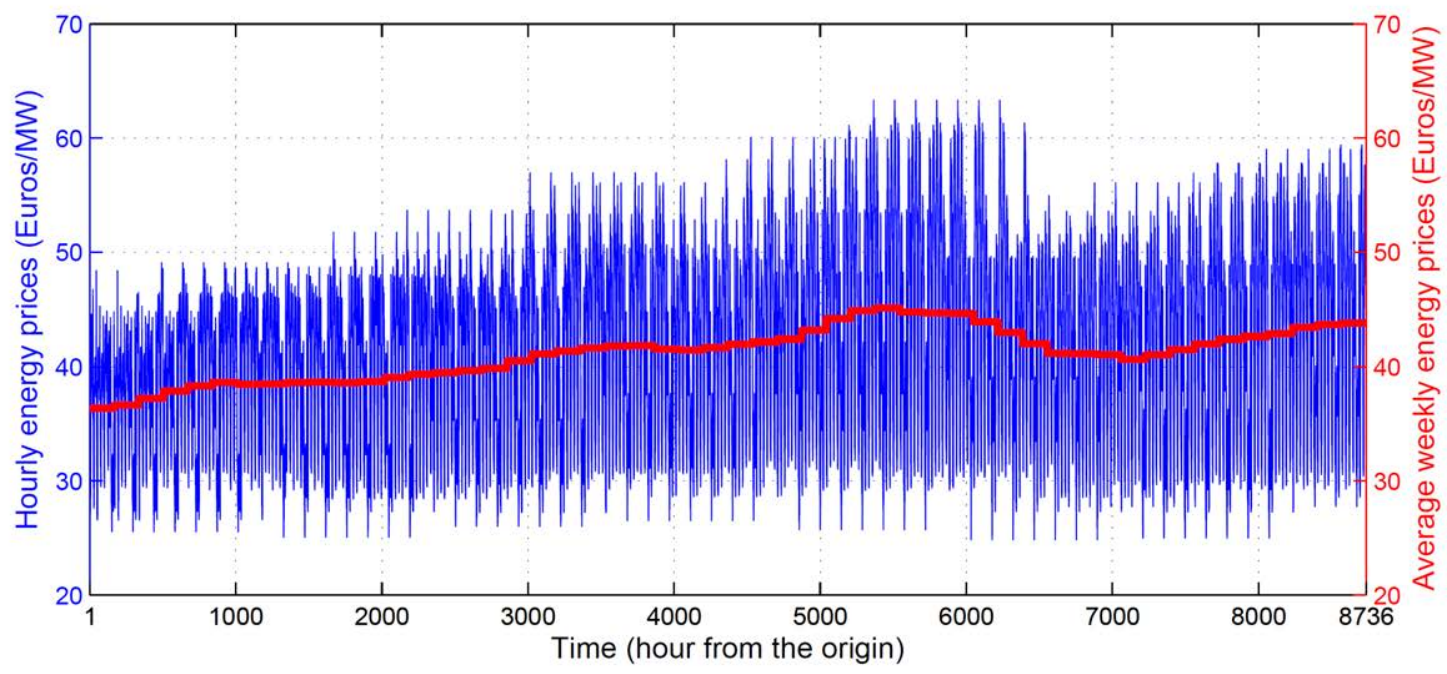

*The origins in study 3 were selected acording to each plant (as discussed in sec. 3.3.1).

Figure B.3.: Hourly and average weekly energy prices of studies 1 to 3 .

(a)

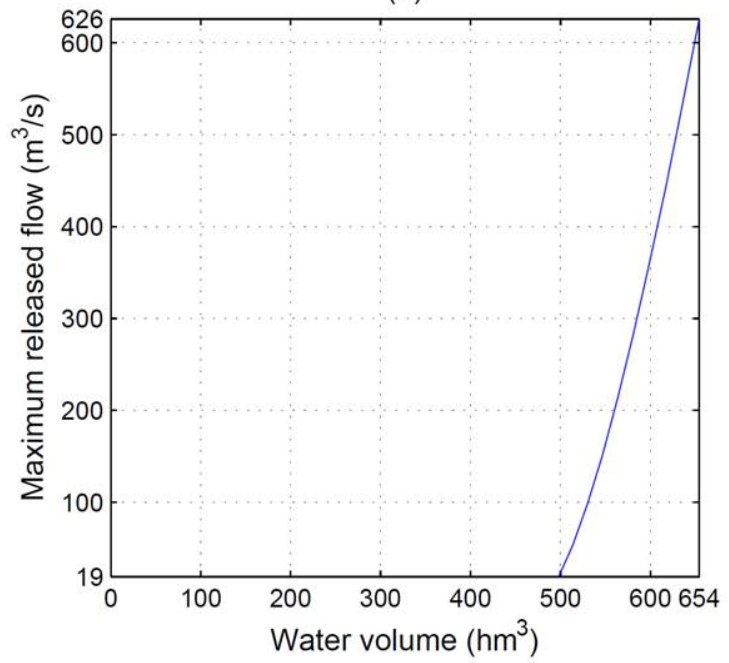

(c)

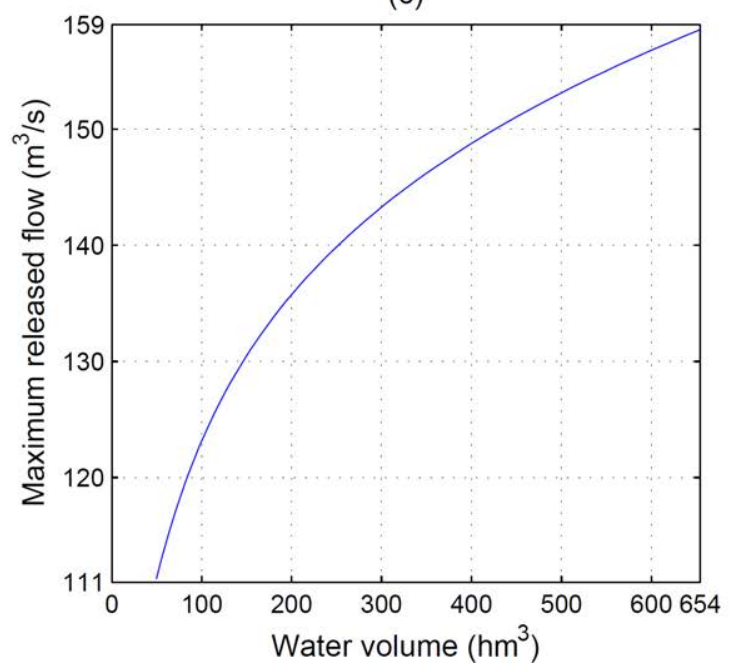

(b)

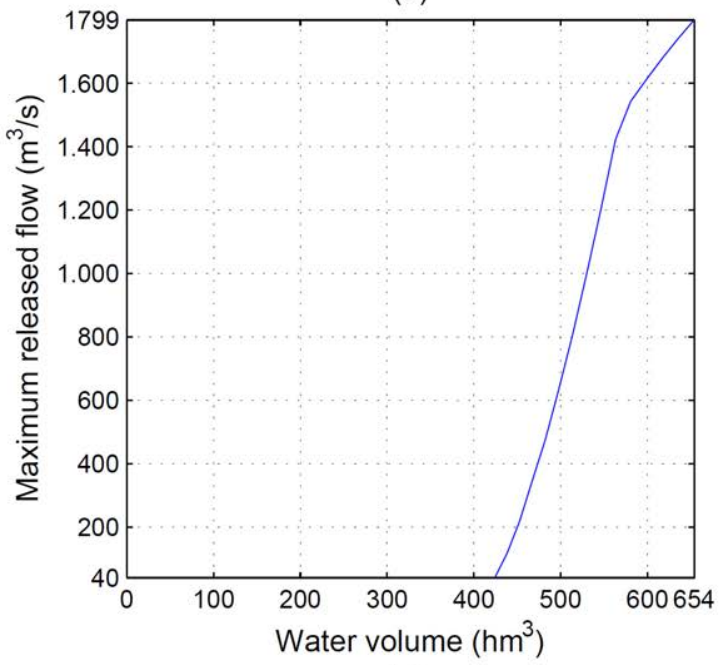

(d)

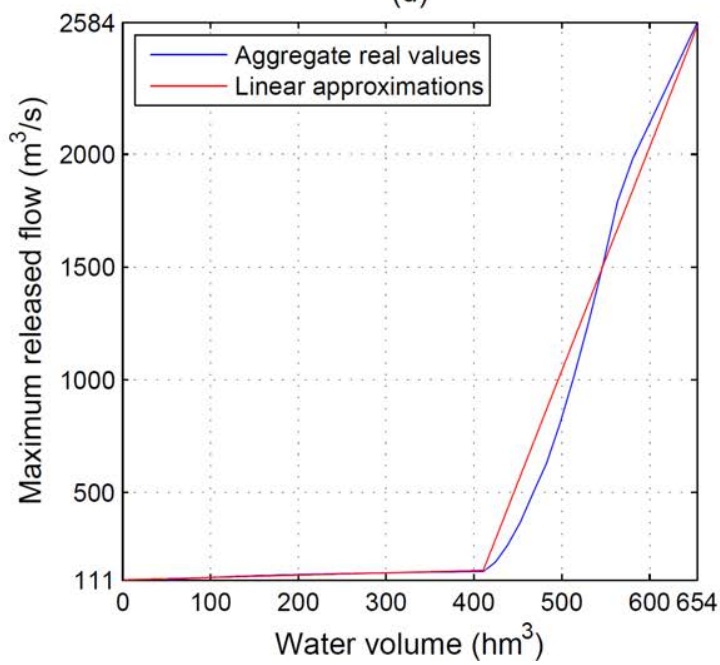

Figure B.4.: Maximum available flows of the bottom outlets and spillways of the plant I: (a) Right spillway; (b) Left spillway; (c) Bottom outlets; (d) Aggregate real values vs. Linear approximations. 
(a)

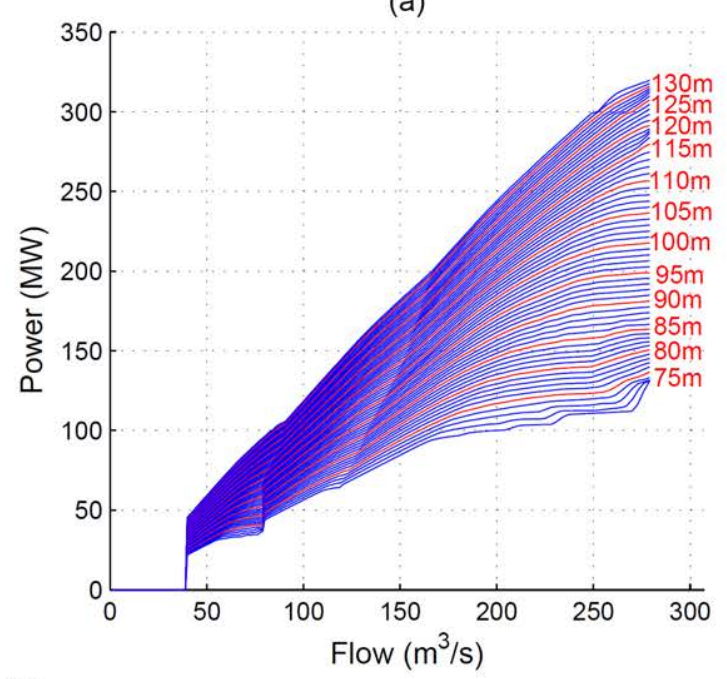

(b)

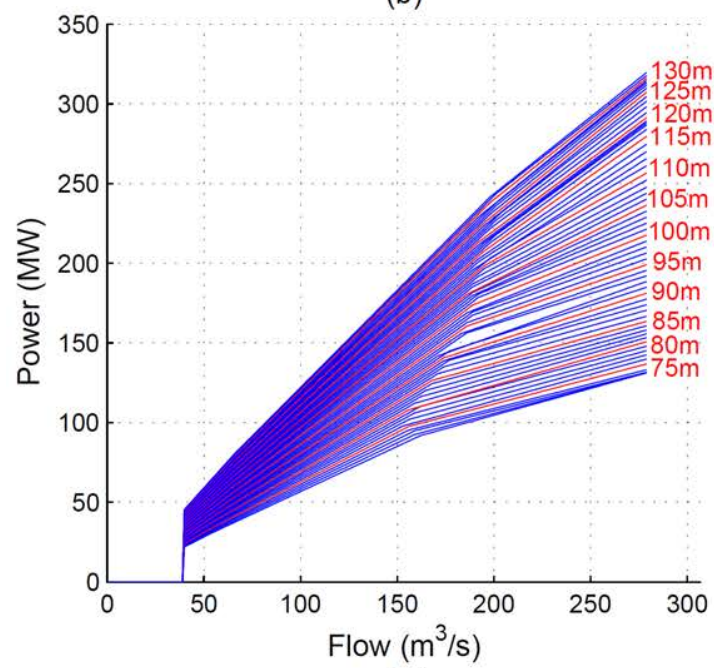

(d)

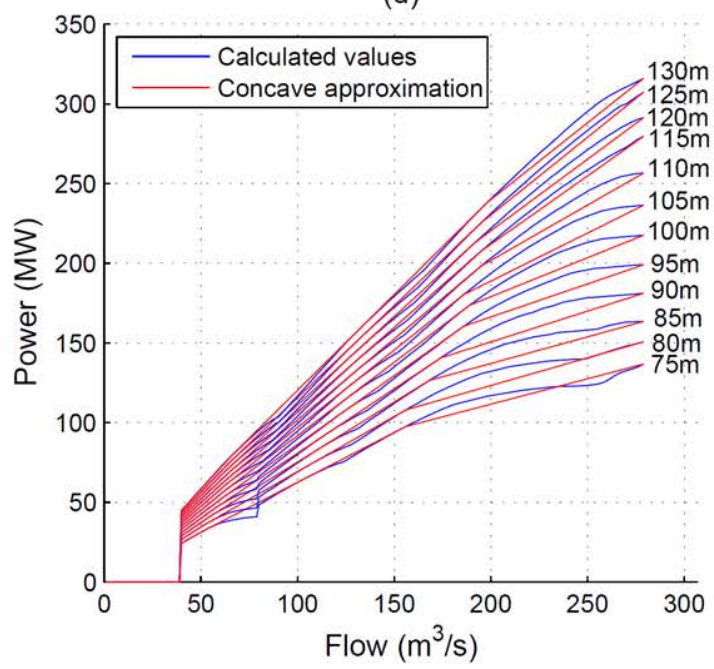

(c)

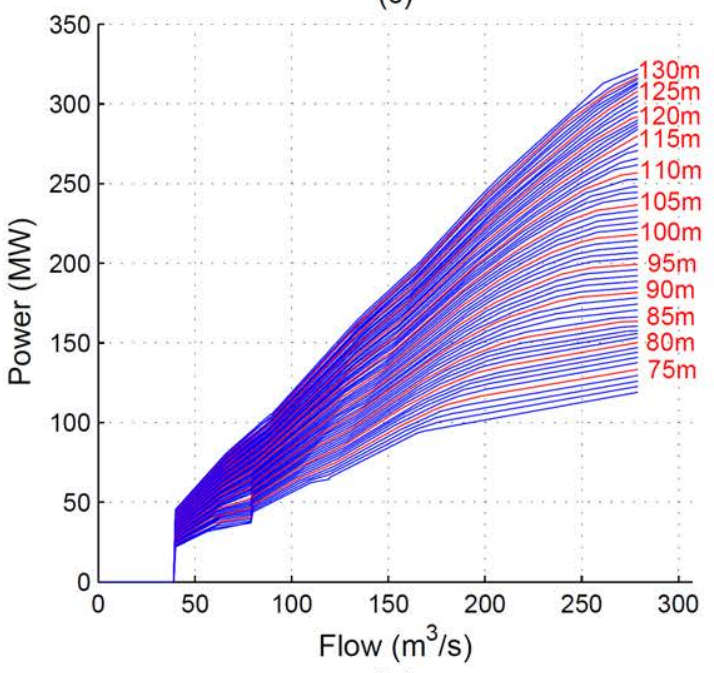

(e)

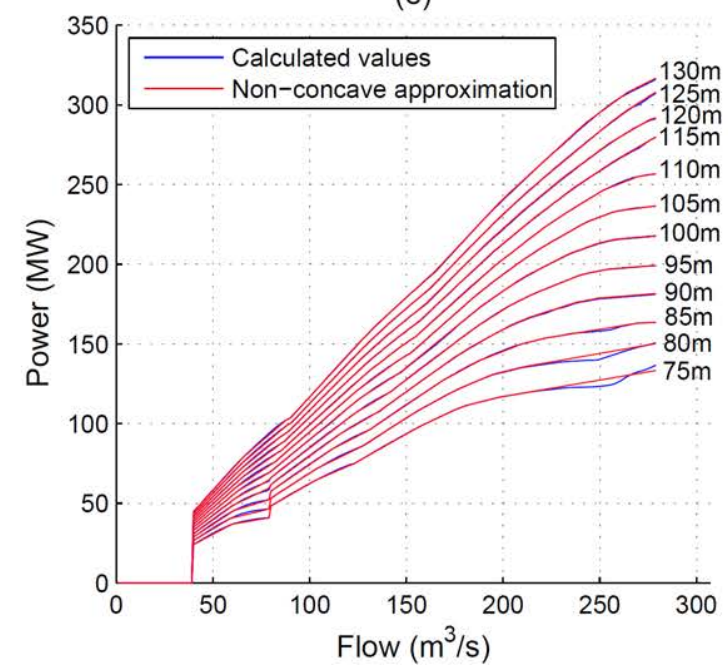

Figure B.5.: Performance curves without hydraulic losses of the plant I: (a) Calculated values; (b) Linear concave approximation; (c) Linear non-concave approximation; (d) Calculated values vs. Linear concave approximation; (e) Calculated values vs. Linear nonconcave approximation. 
(a)

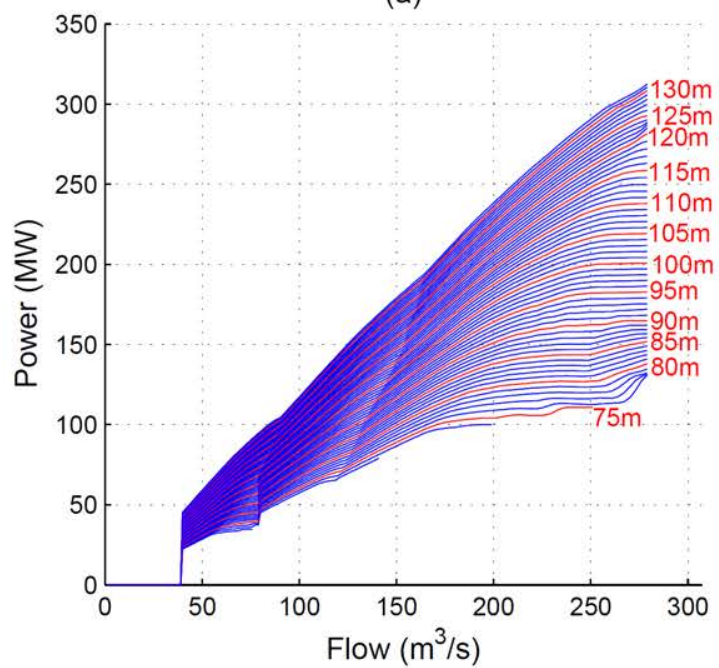

(b)

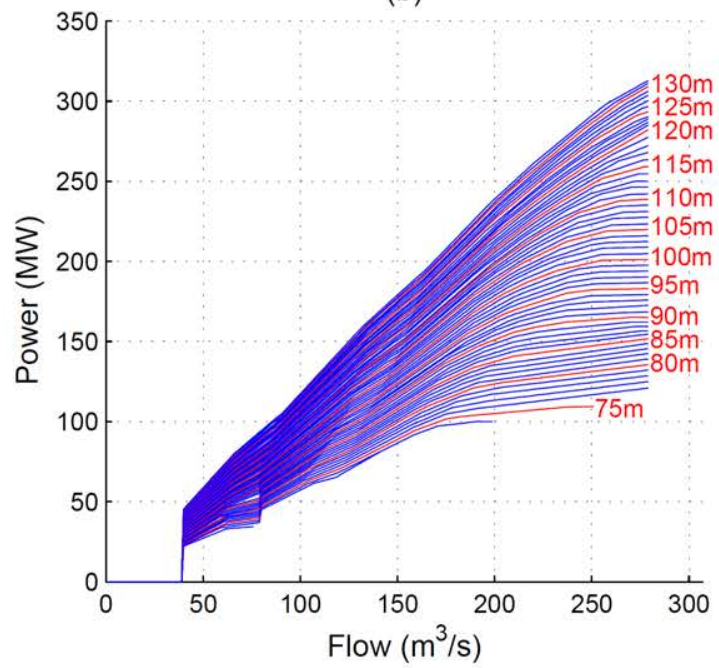

(c)

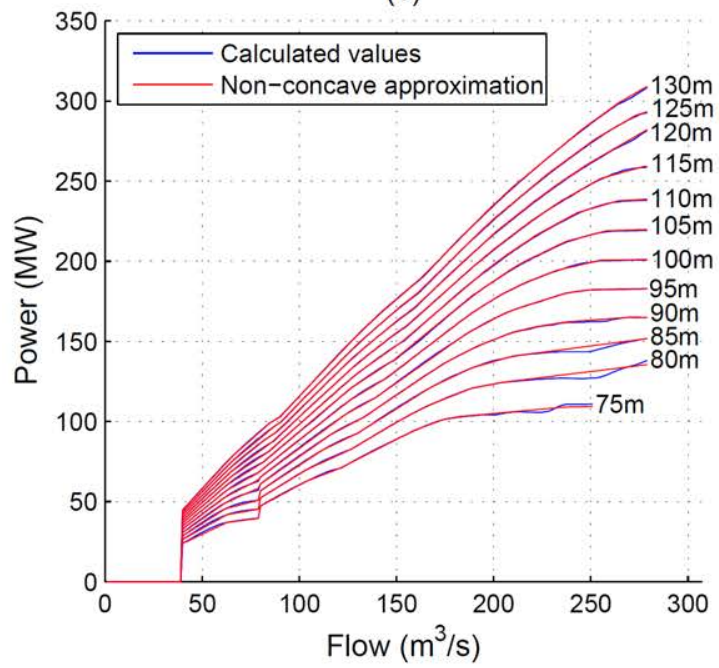

Figure B.6.: Performance curves with hydraulic losses of the plant I: (a) Calculated values; (b) Linear concave approximation; (c) Calculated values vs. Linear concave approximation. 
(a)

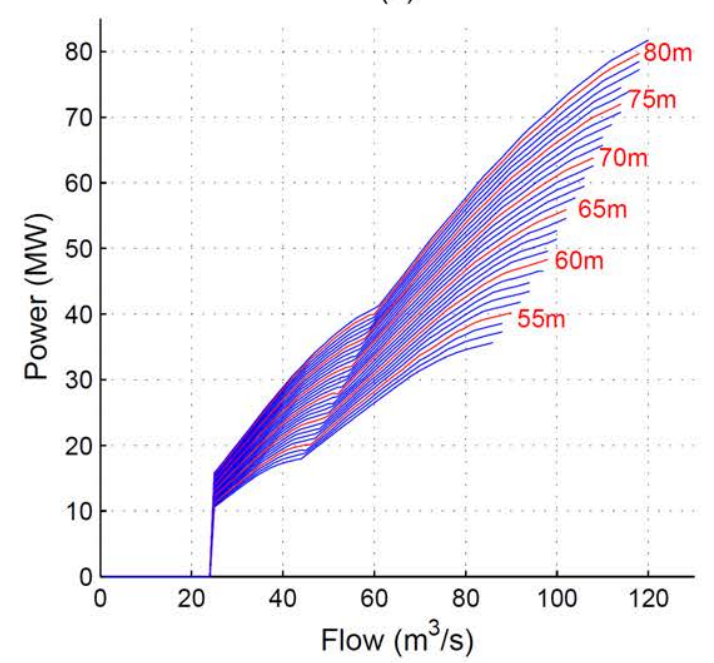

(b)

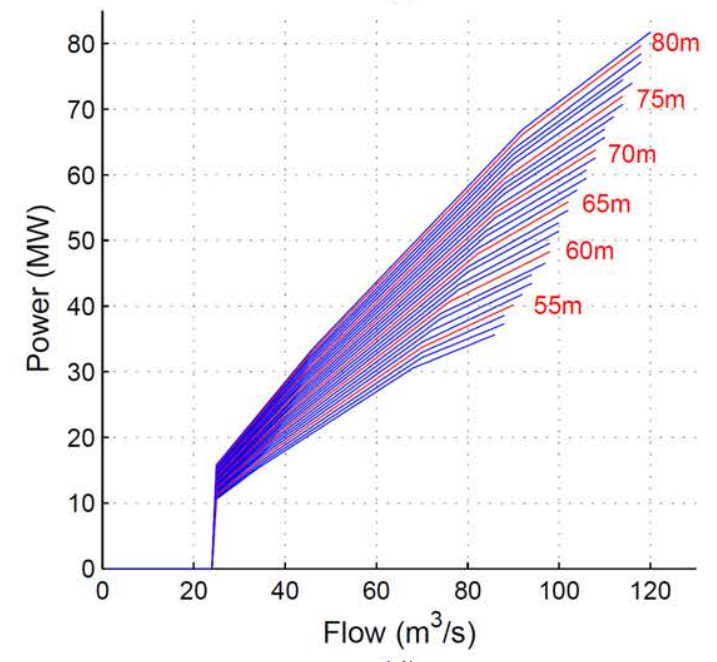

(d)

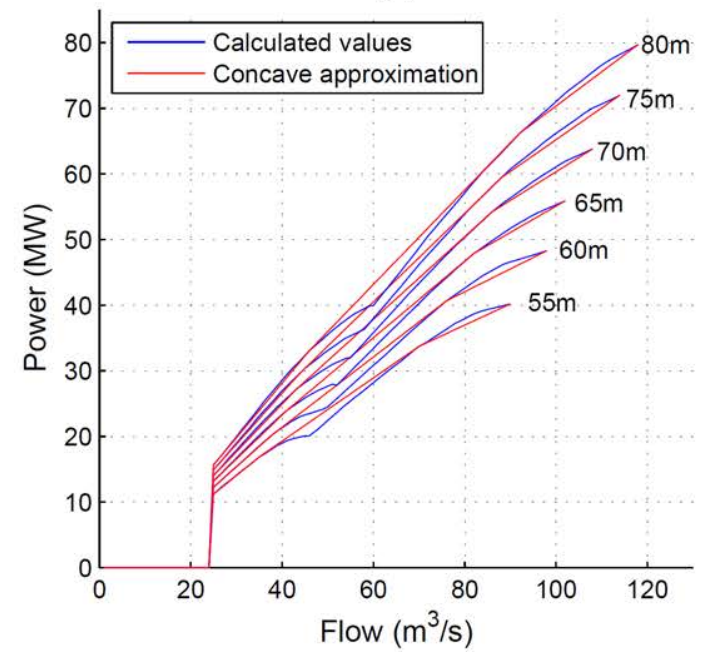

(c)

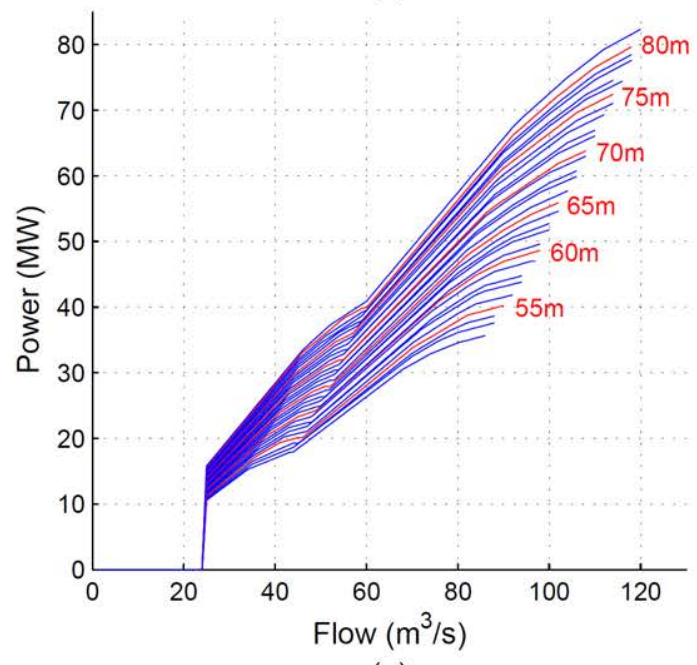

(e)

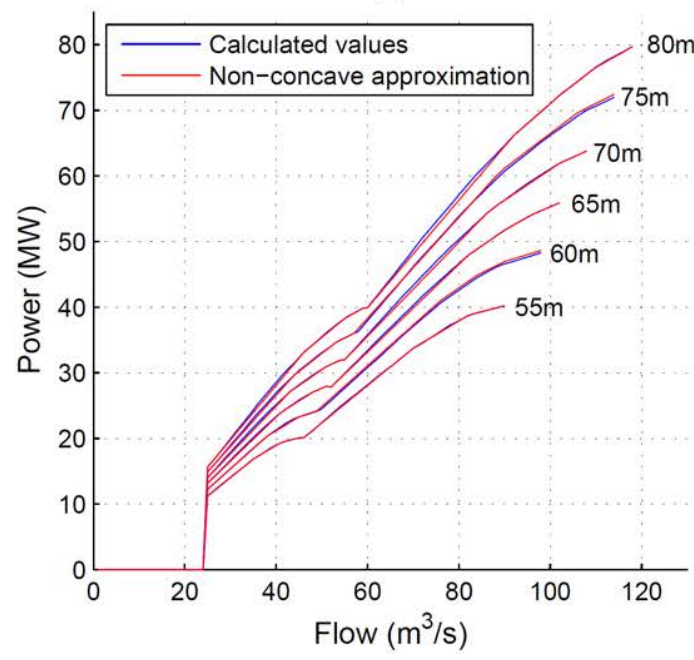

Figure B.7.: Performance curves without hydraulic losses of the plant II: (a) Calculated values; (b) Linear concave approximation; (c) Linear non-concave approximation; (d) Calculated values vs. Linear concave approximation; (e) Calculated values vs. Linear nonconcave approximation. 
(a)

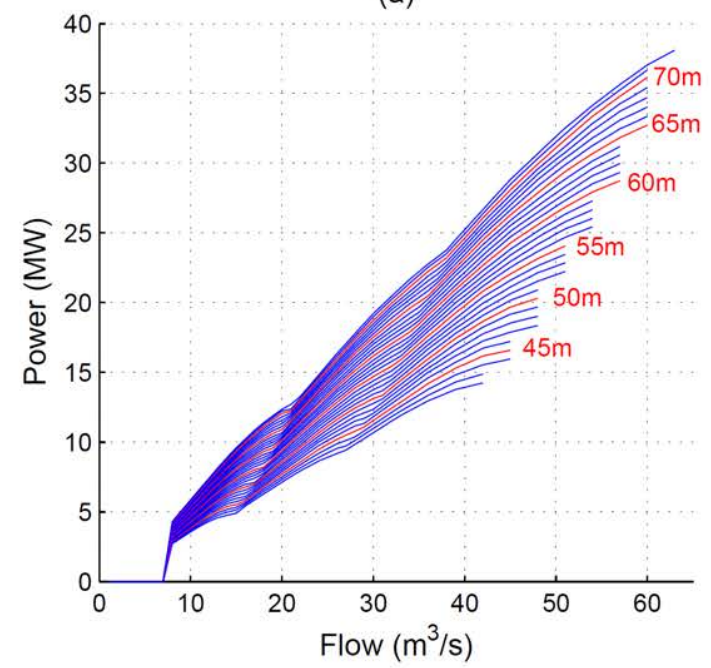

(b)

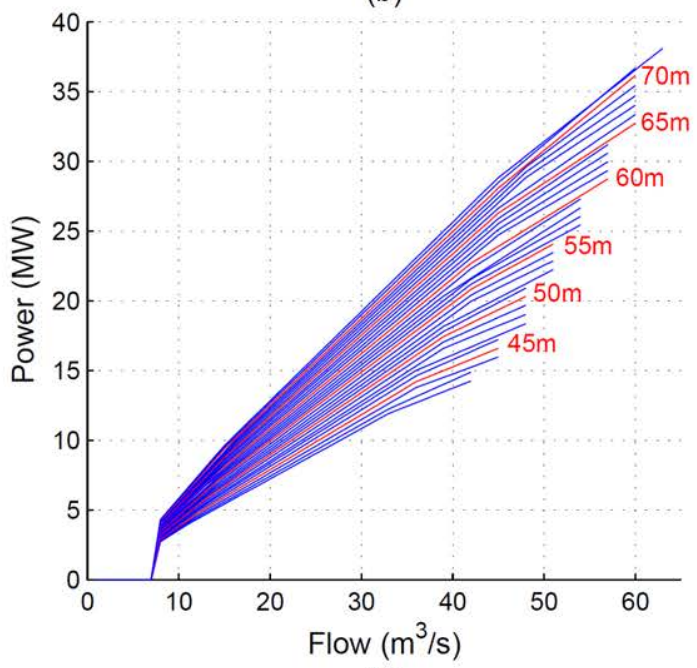

(d)

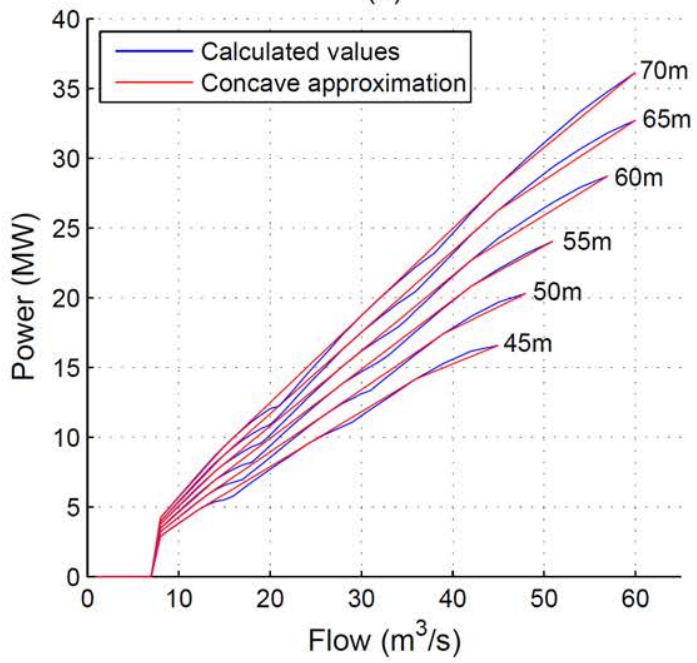

(c)

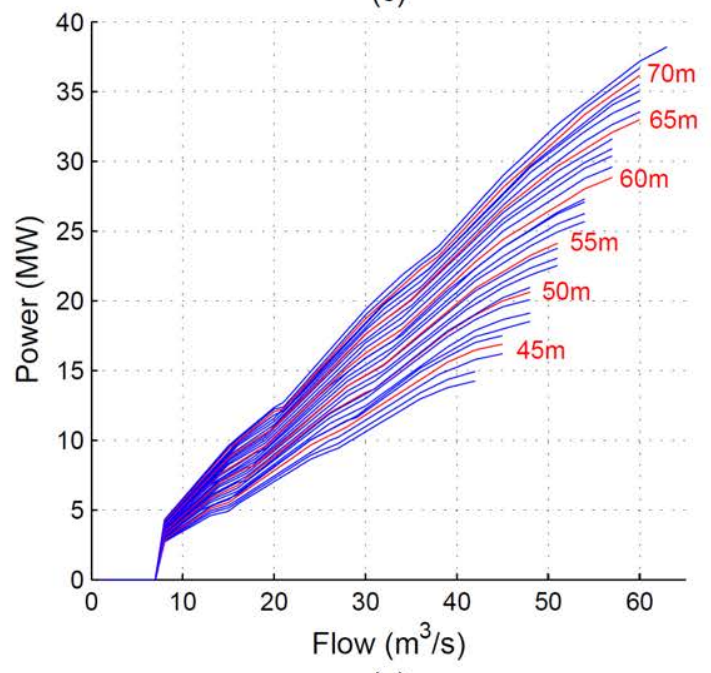

(e)

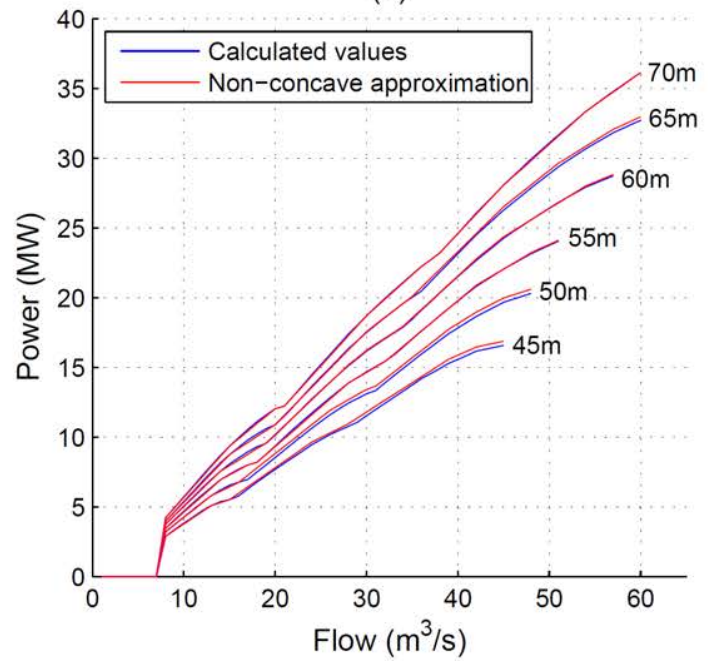

Figure B.8.: Performance curves without hydraulic losses of the plant III: (a) Calculated values; (b) Linear concave approximation; (c) Linear non-concave approximation; (d) Calculated values vs. Linear concave approximation; (e) Calculated values vs. Linear nonconcave approximation. 
(a)

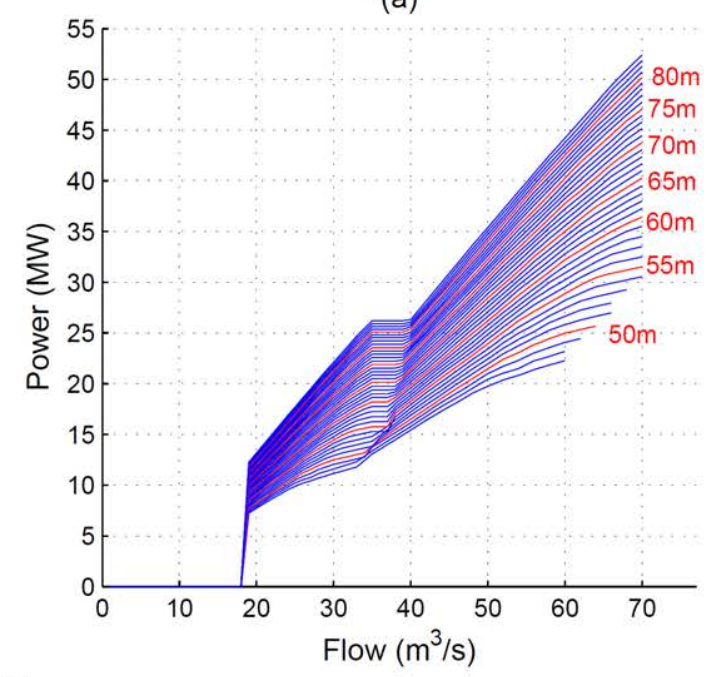

(b)

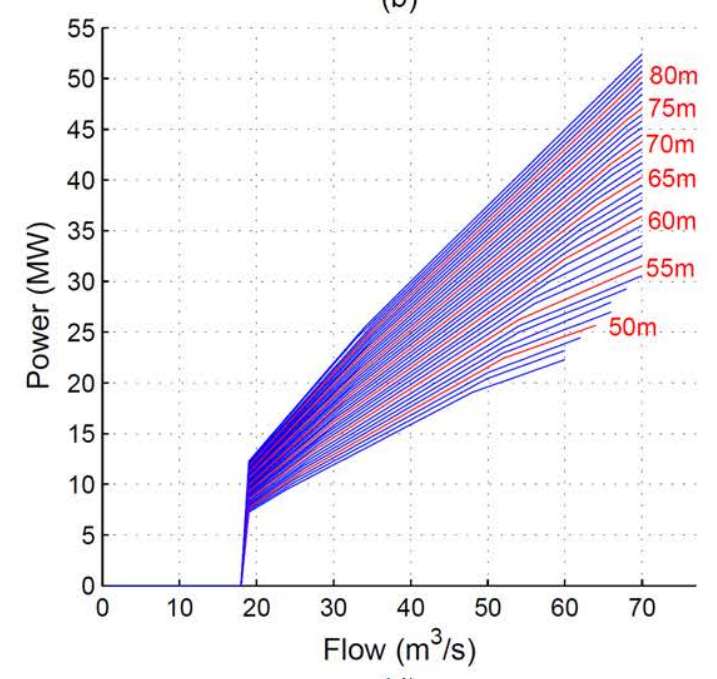

(d)

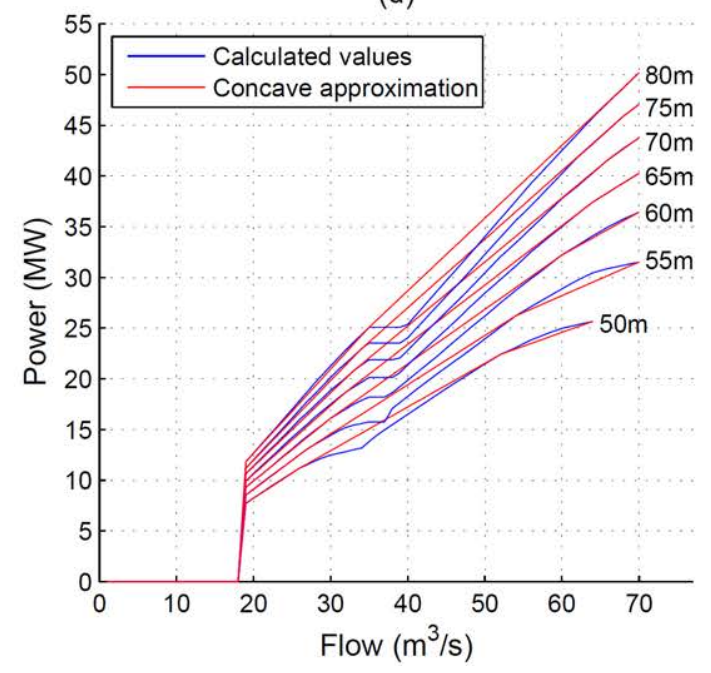

(c)

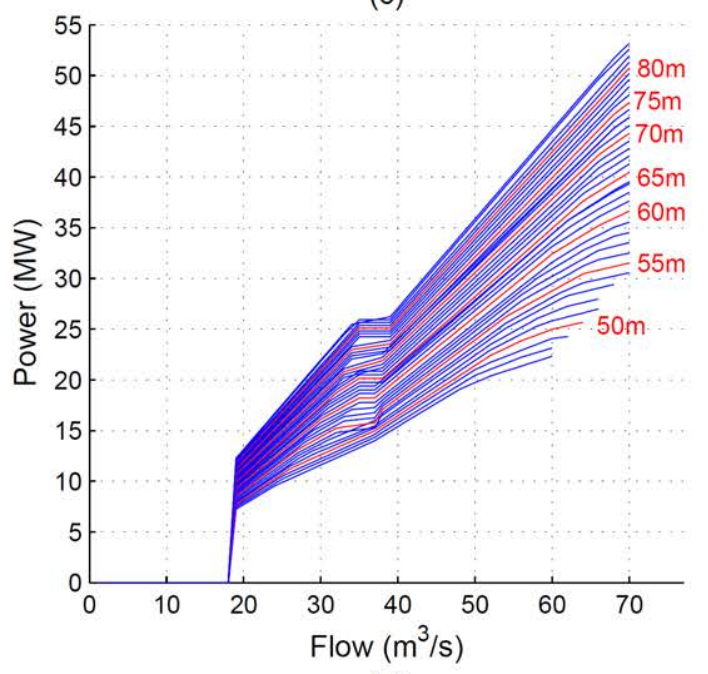

(e)

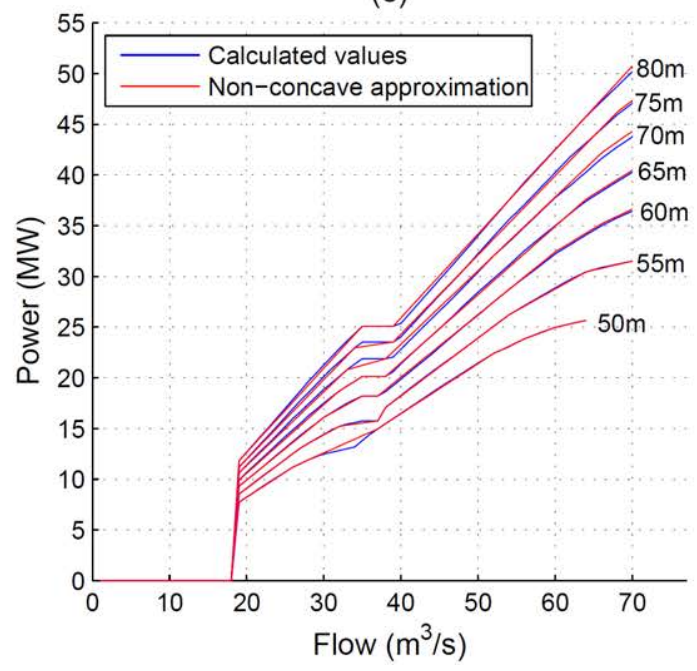

Figure B.9.: Performance curves without hydraulic losses of the plant IV: (a) Calculated values; (b) Linear concave approximation; (c) Linear non-concave approximation; (d) Calculated values vs. Linear concave approximation; (e) Calculated values vs. Linear nonconcave approximation. 
(a)

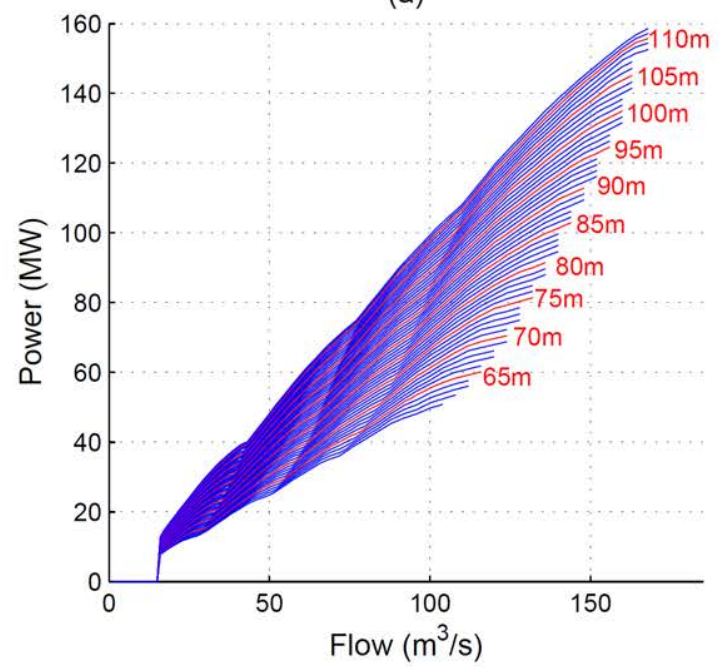

(b)

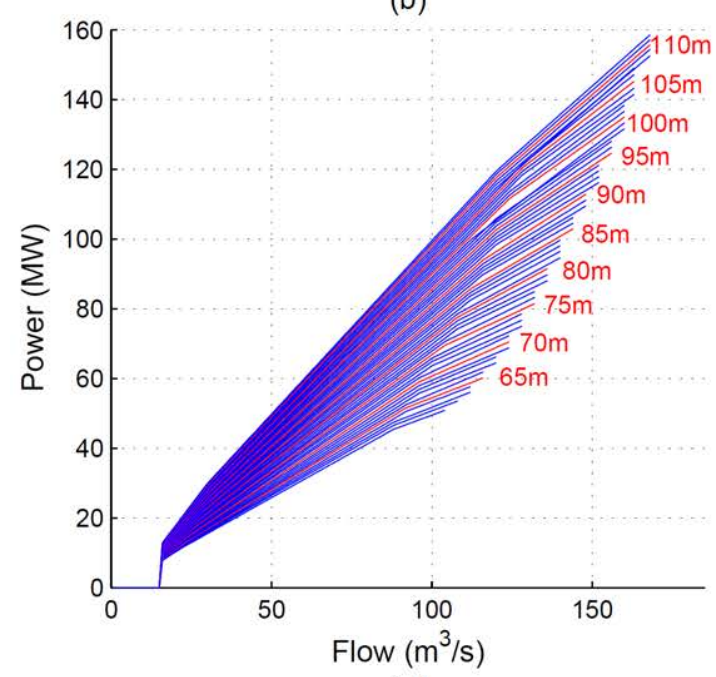

(d)

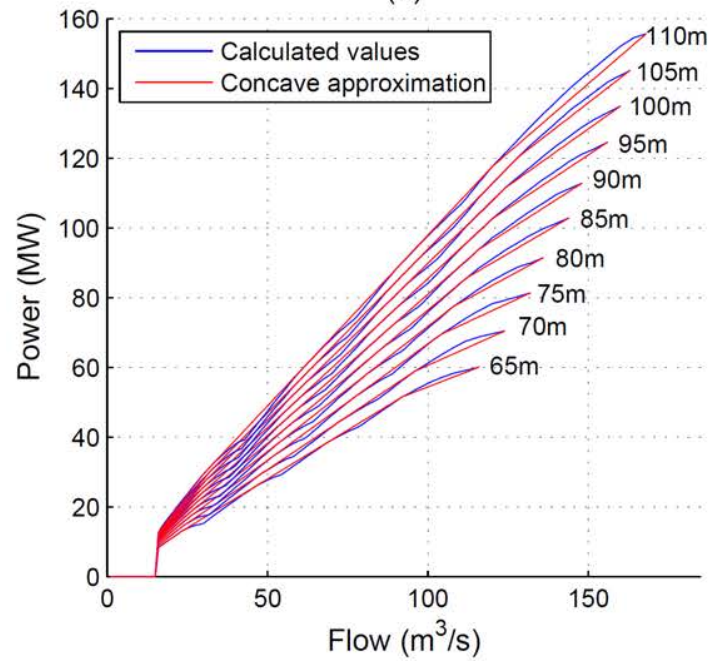

(c)

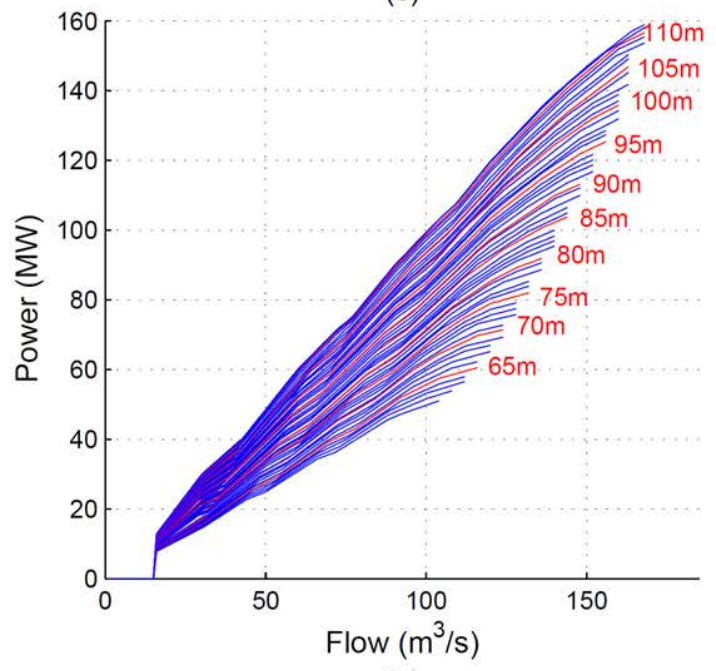

(e)

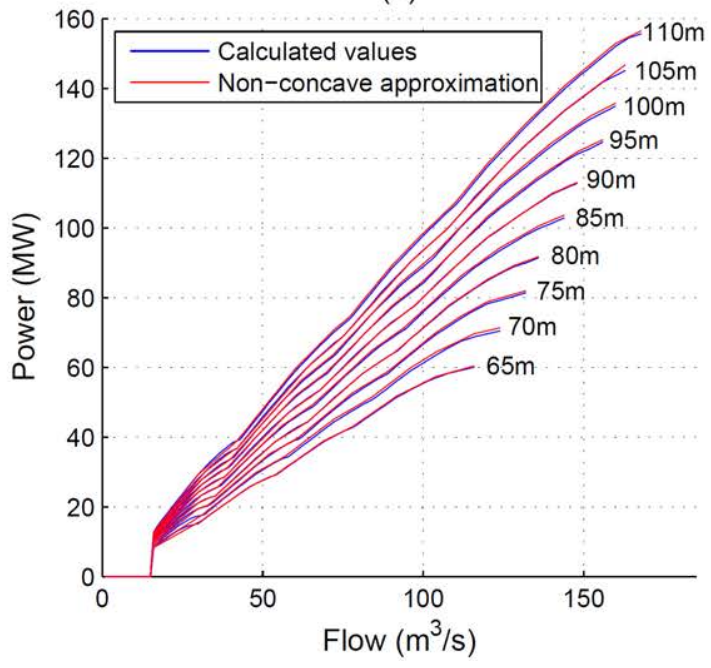

Figure B.10.: Performance curves without hydraulic losses of the plant V: (a) Calculated values; (b) Linear concave approximation; (c) Linear non-concave approximation; (d) Calculated values vs. Linear concave approximation; (e) Calculated values vs. Linear nonconcave approximation. 
(a)

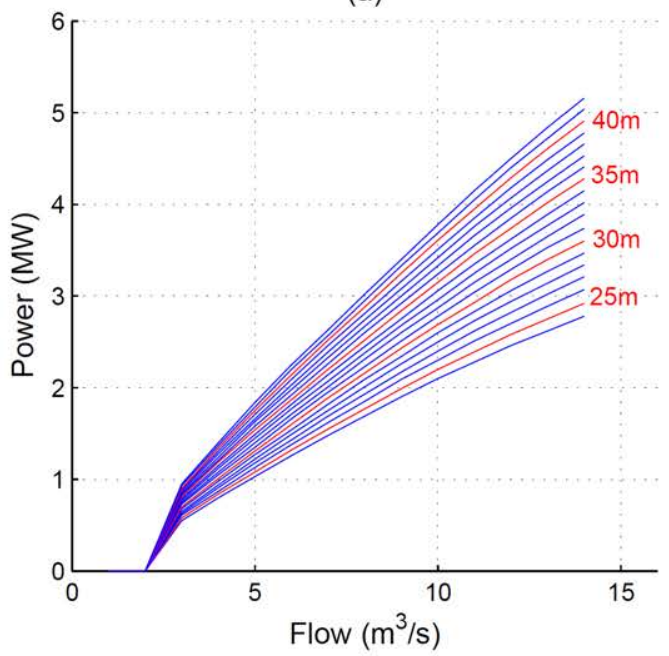

(b)

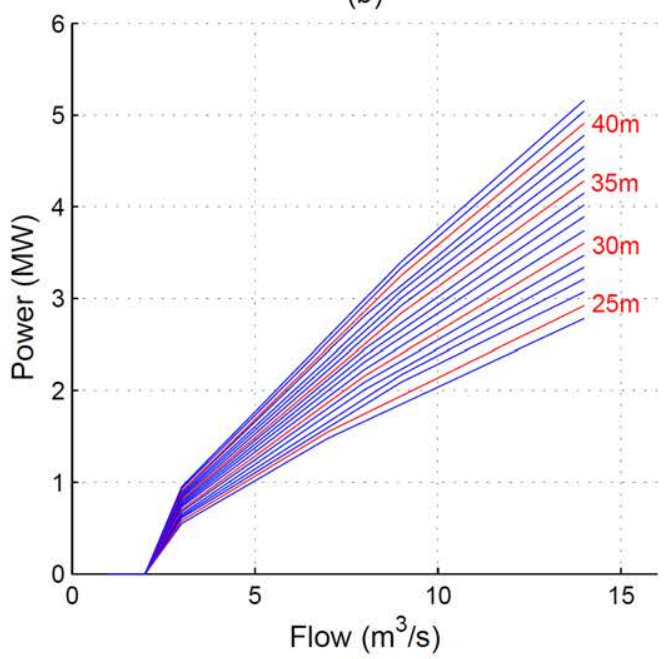

(c)

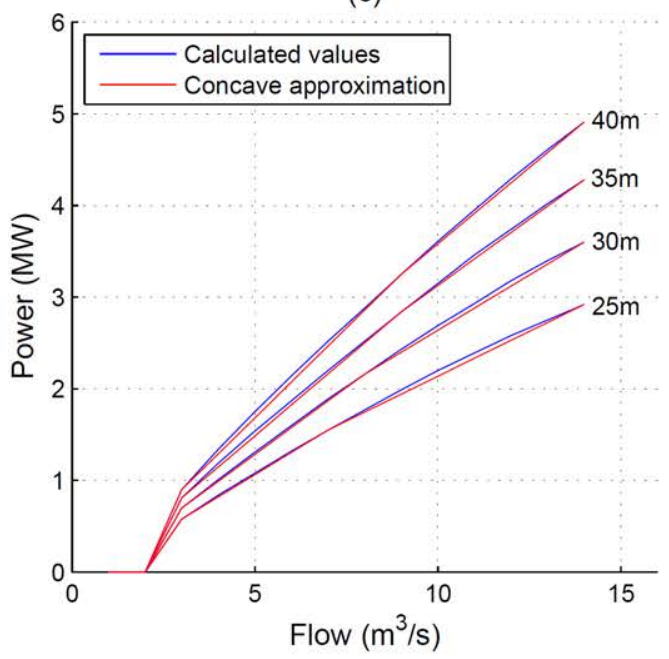

Figure B.11.: Performance curves without hydraulic losses of the plant VI: (a) Calculated values; (b) Linear concave approximation; (c) Calculated values vs. Linear concave approximation. (a)

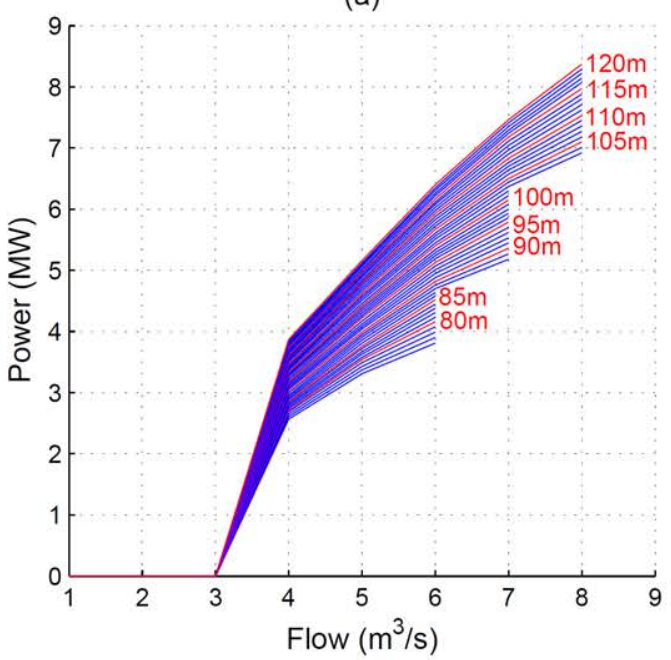

(b)

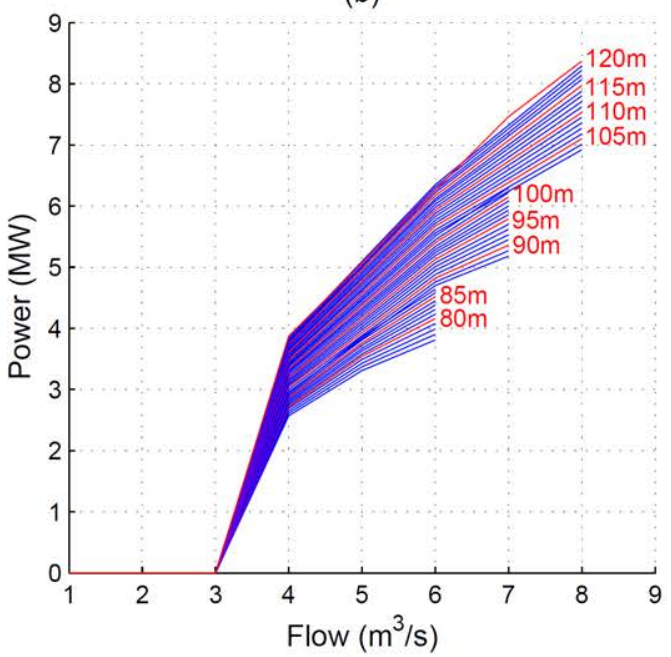

(c)

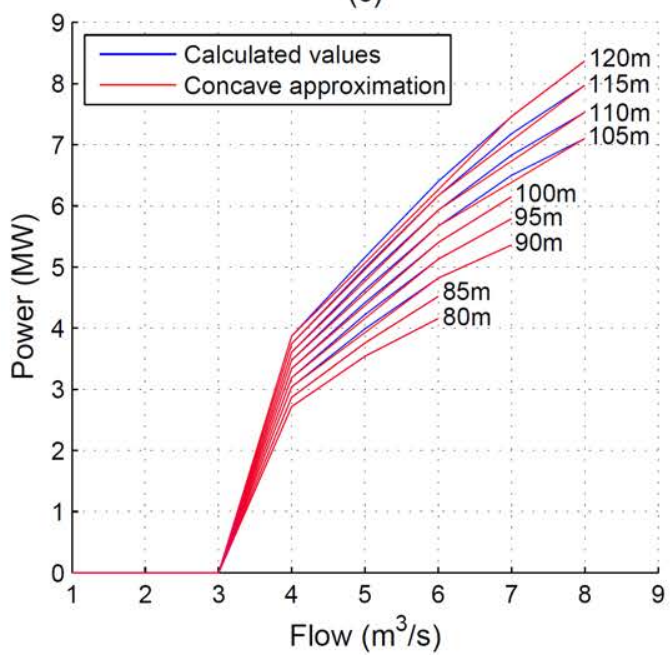

Figure B.12.: Performance curves without hydraulic losses of the plant VII: (a) Calculated values; (b) Linear concave approximation; (c) Calculated values vs. Linear concave approximation. 
(a)

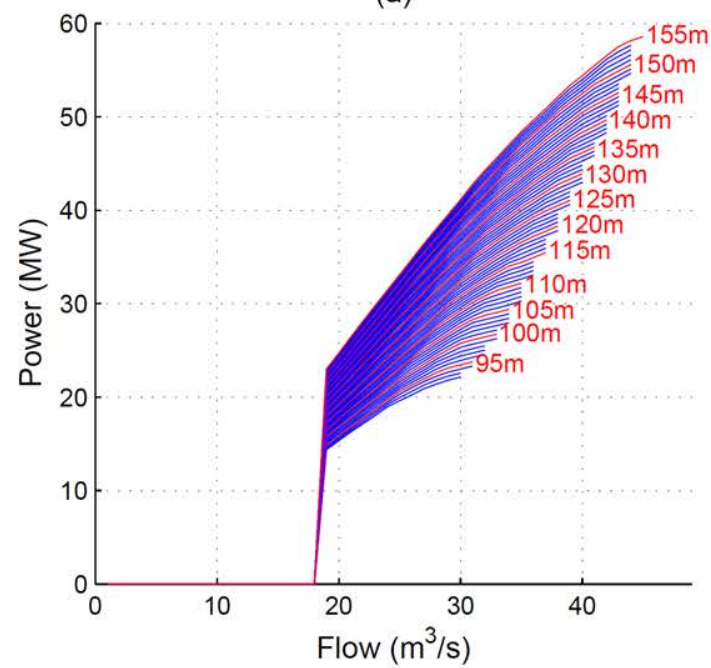

(b)

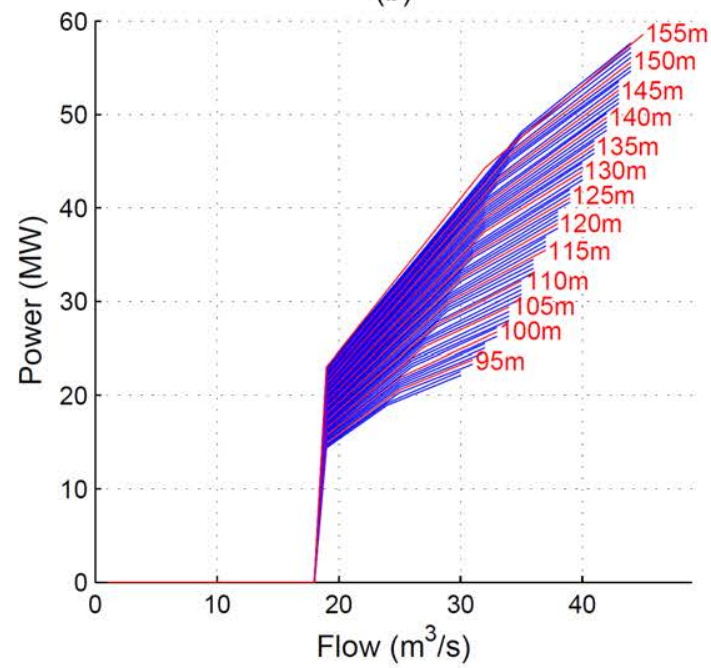

(c)

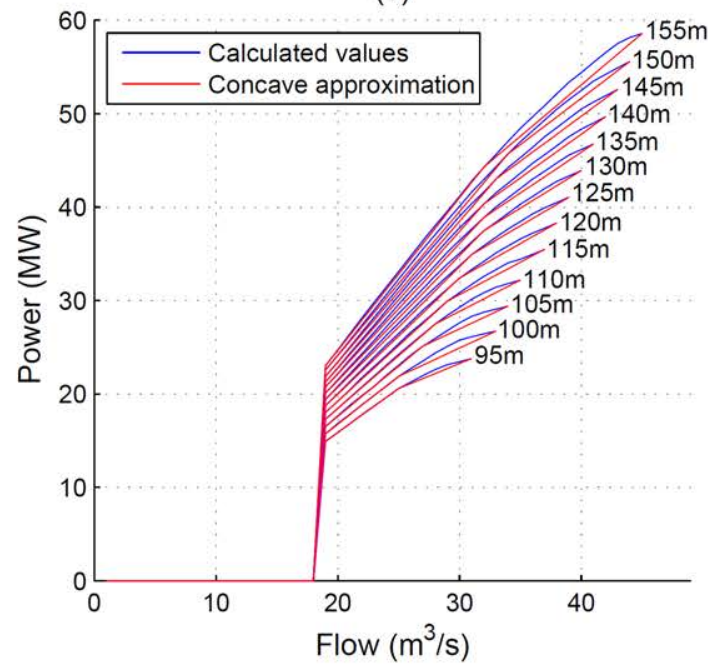

Figure B.13.: Performance curves without hydraulic losses of the plant VIII: (a) Calculated values; (b) Linear concave approximation; (c) Calculated values vs. Linear concave approximation. 
(a)

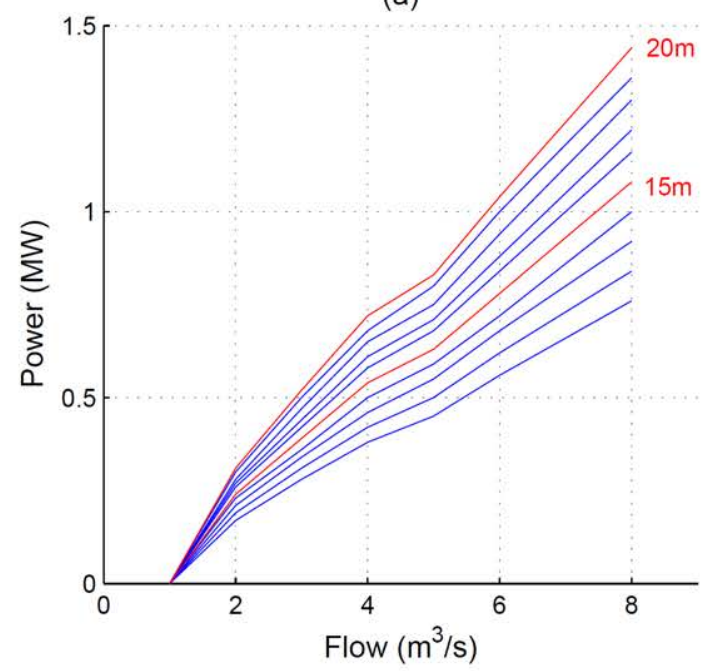

(b)

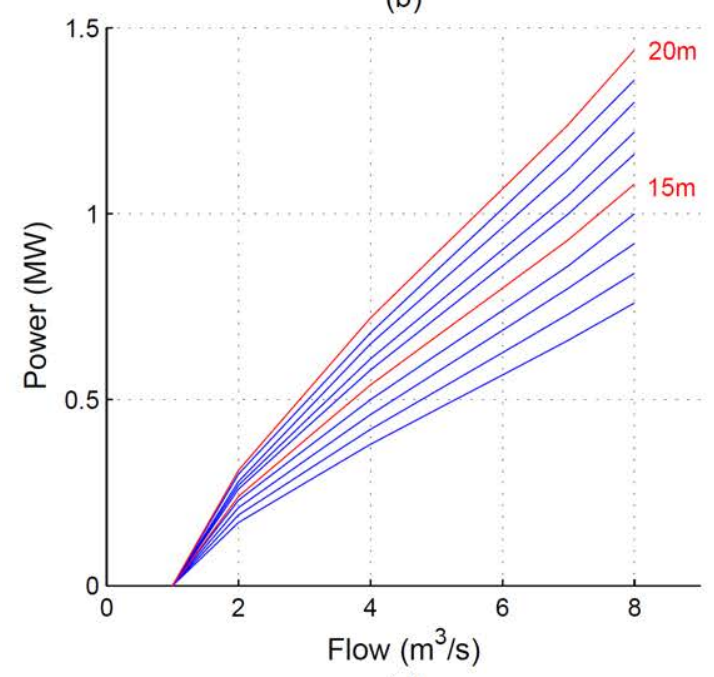

(d)

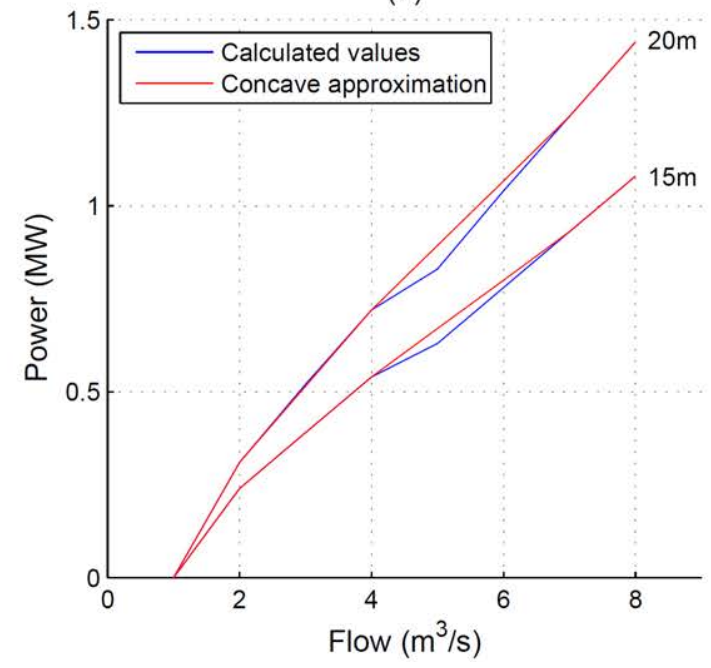

(c)

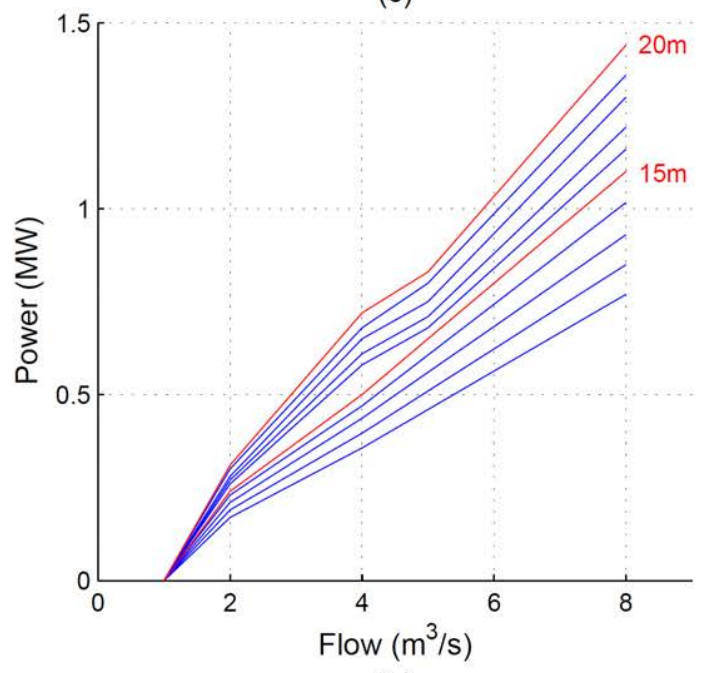

(e)

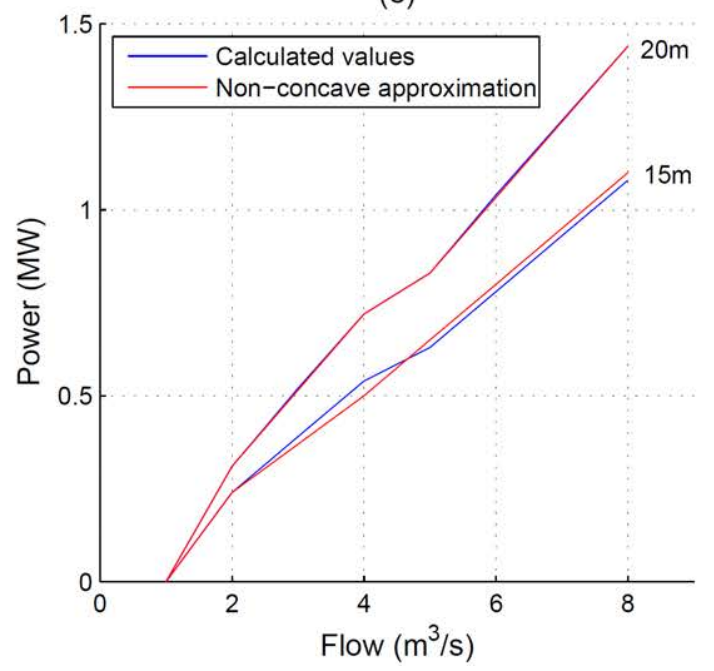

Figure B.14.: Performance curves without hydraulic losses of the plant IX: (a) Calculated values; (b) Linear concave approximation; (c) Linear non-concave approximation; (d) Calculated values vs. Linear concave approximation; (e) Calculated values vs. Linear nonconcave approximation. 
(a)

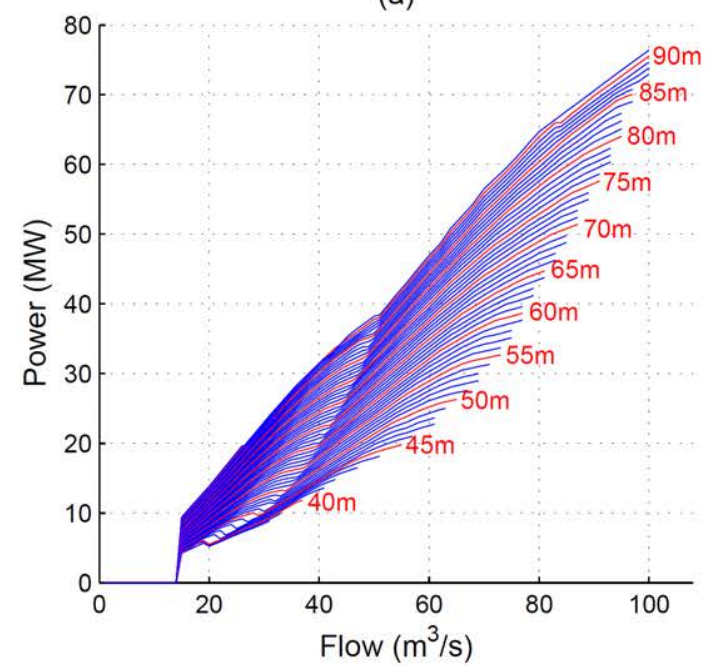

(b)

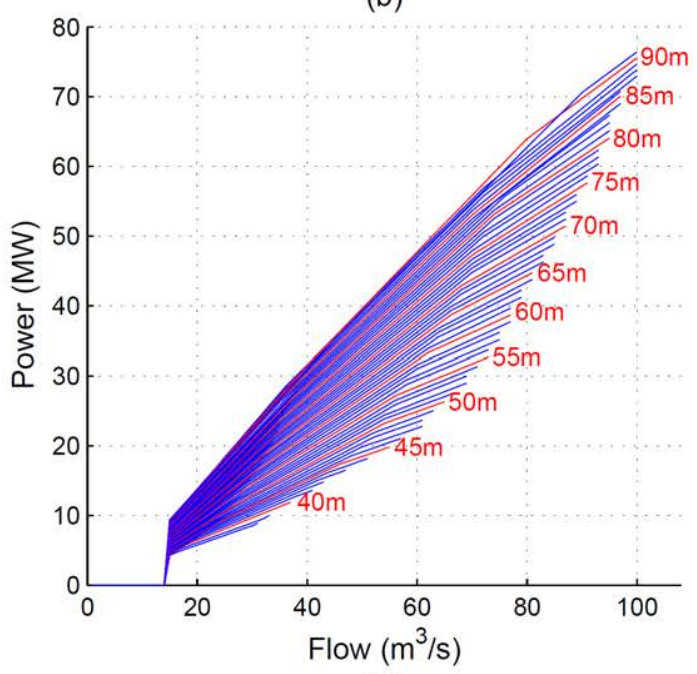

(d)

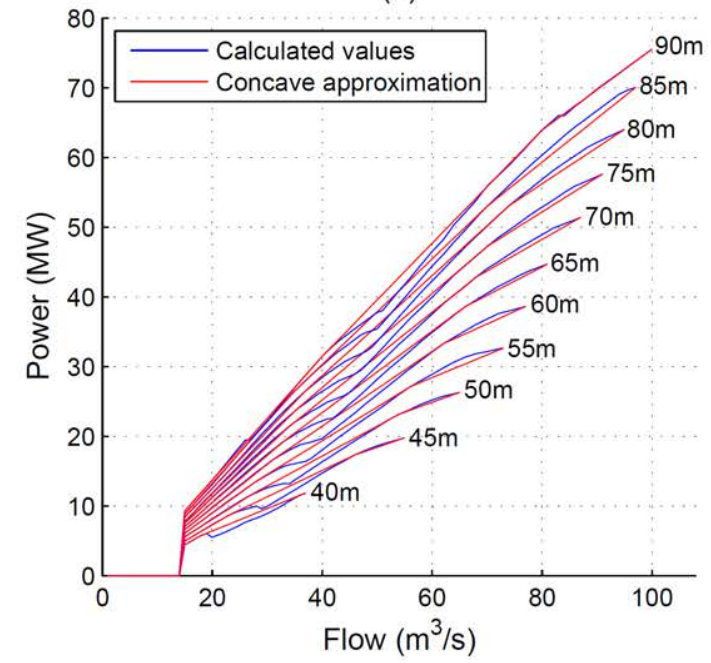

(c)

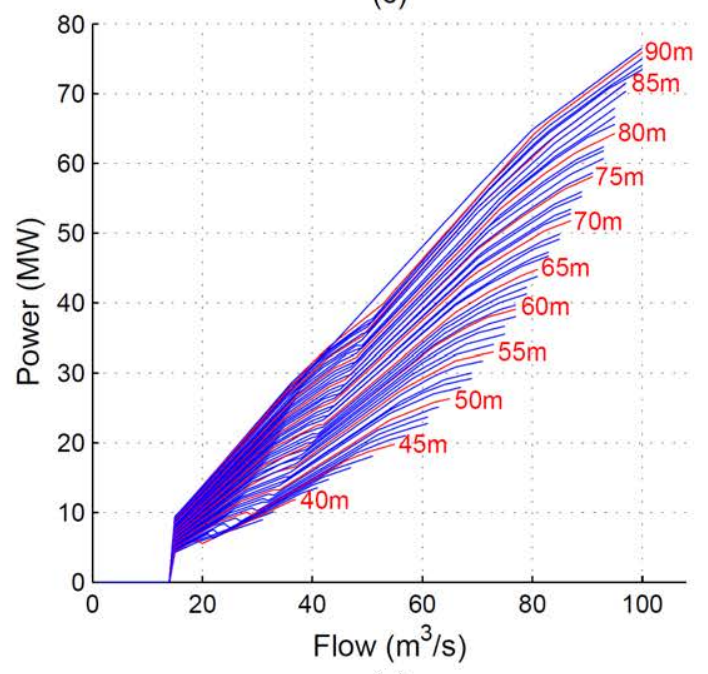

(e)

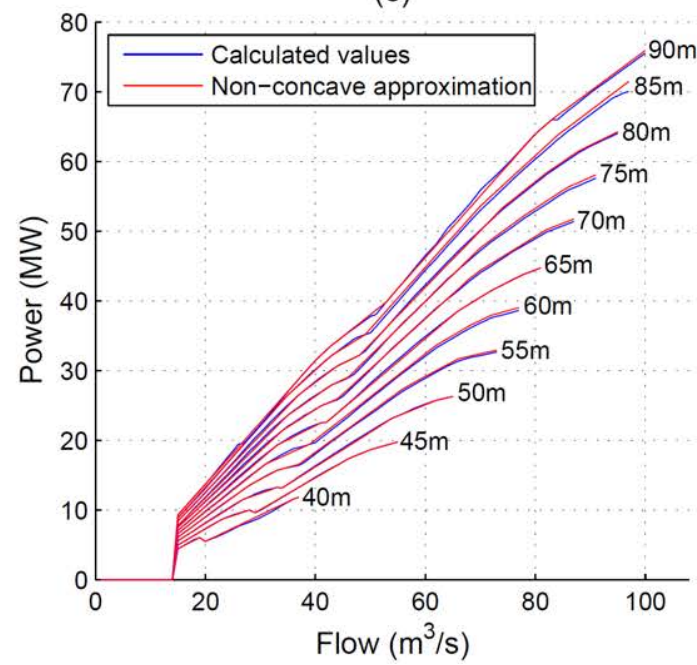

Figure B.15.: Performance curves without hydraulic losses of the plant X: (a) Calculated values; (b) Linear concave approximation; (c) Linear non-concave approximation; (d) Calculated values vs. Linear concave approximation; (e) Calculated values vs. Linear nonconcave approximation. 
(a)

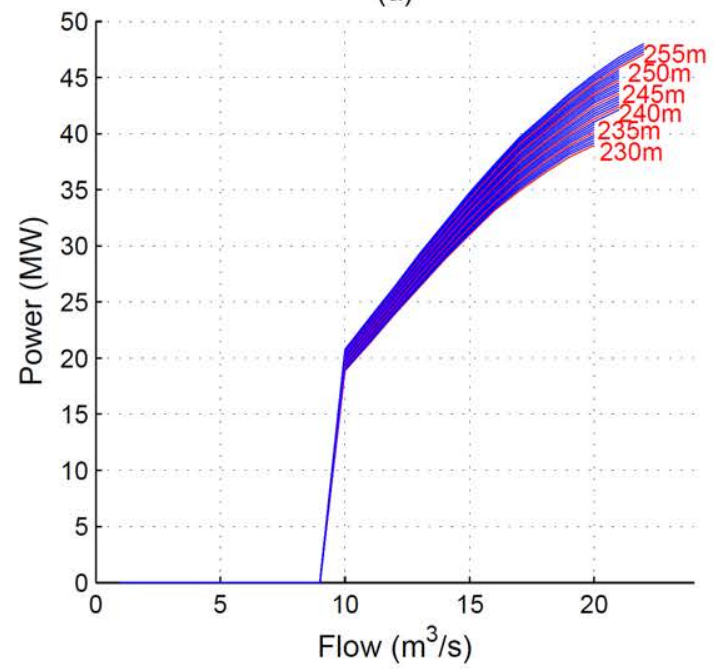

(b)

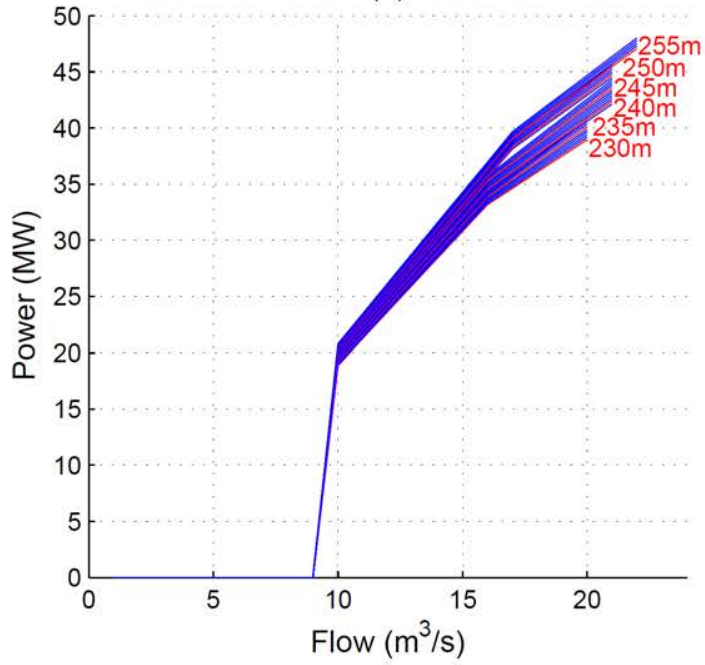

(c)

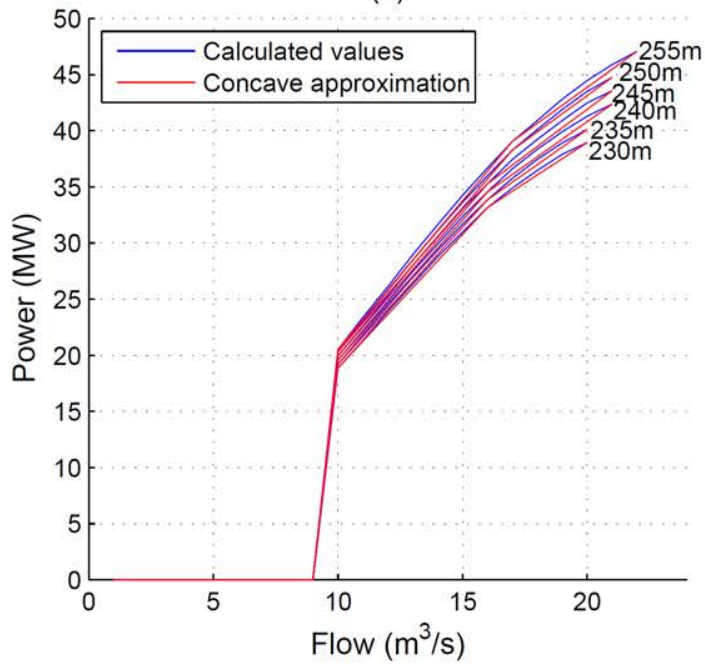

Figure B.16.: Performance curves without hydraulic losses of the plant A: (a) Calculated values; (b) Linear concave approximation; (c) Calculated values vs. Linear concave approximation. 
(a)

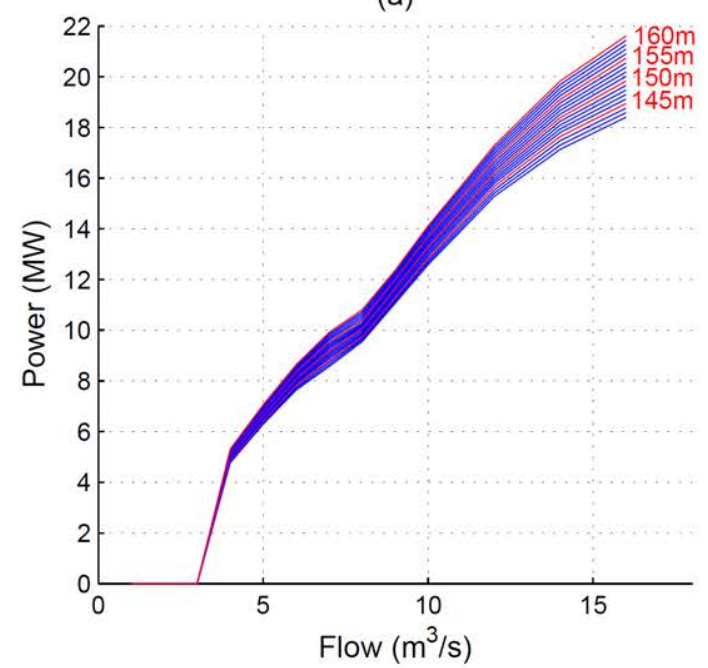

(b)

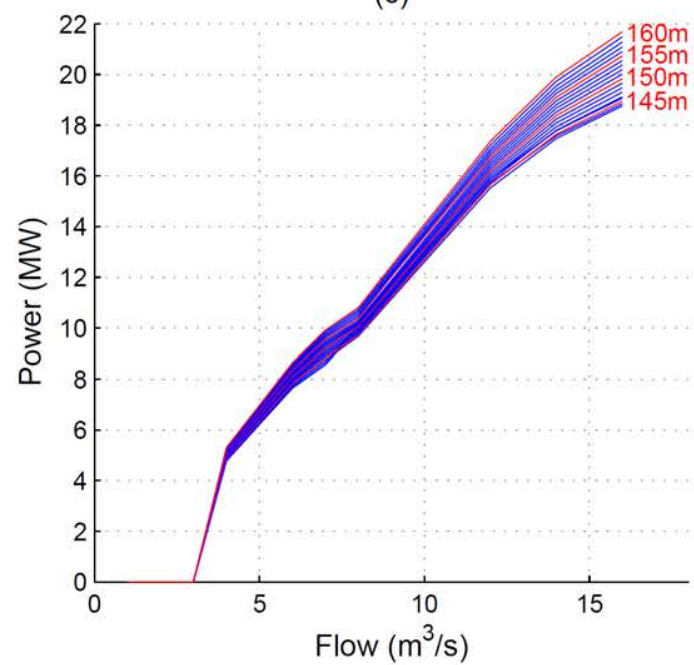

(e)
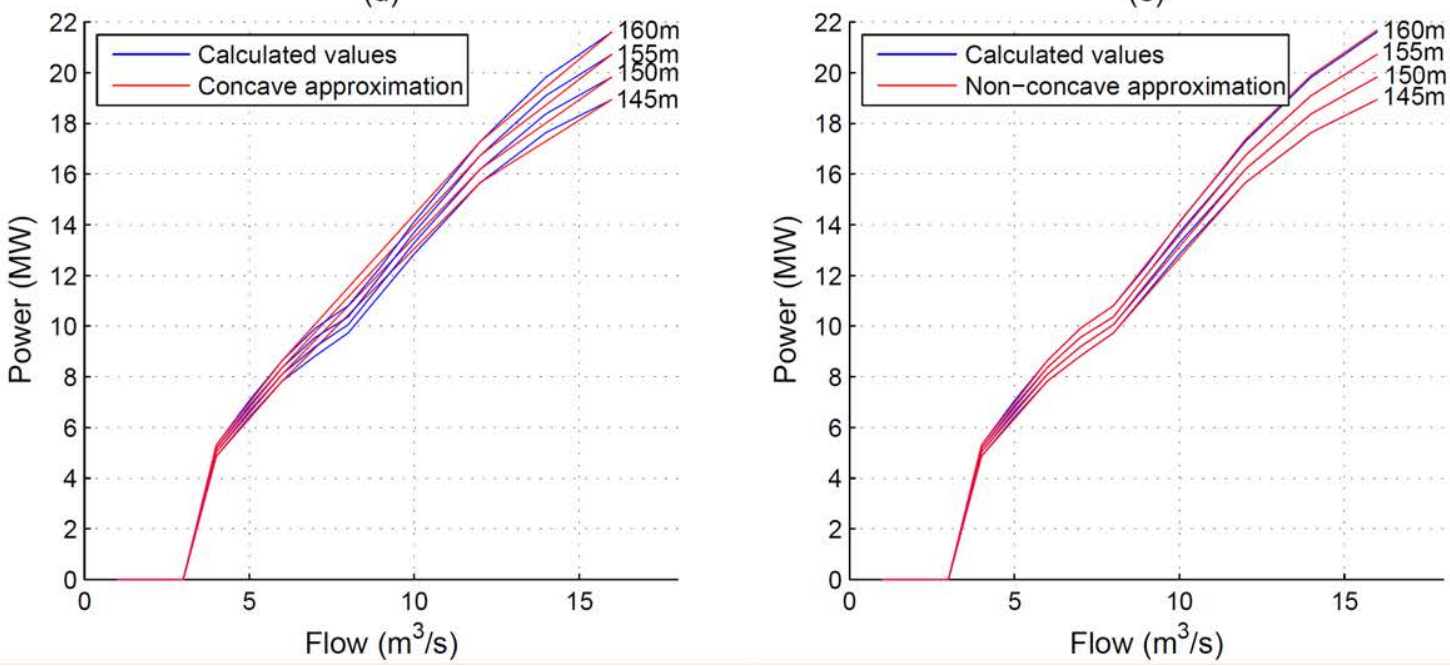

Figure B.17.: Performance curves without hydraulic losses of the plant B: (a) Calculated values; (b) Linear concave approximation; (c) Linear non-concave approximation; (d) Calculated values vs. Linear concave approximation; (e) Calculated values vs. Linear nonconcave approximation. 
(a)

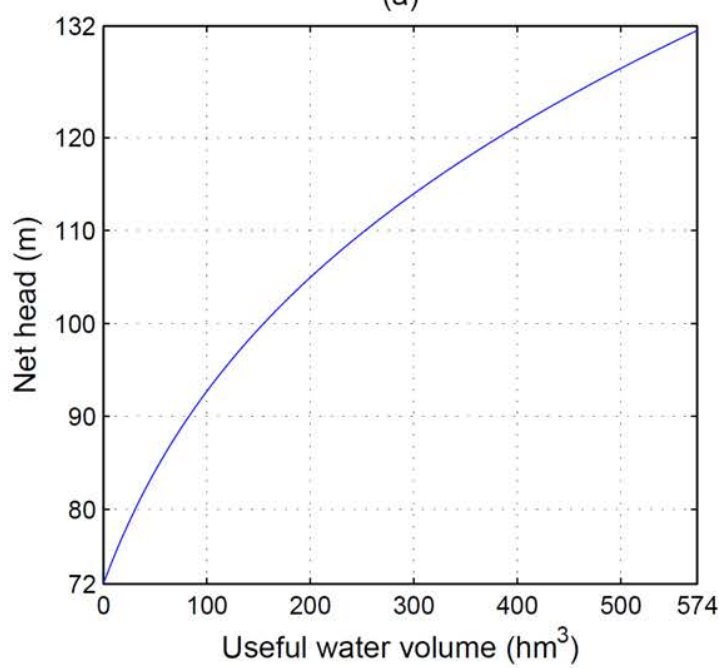

(c)

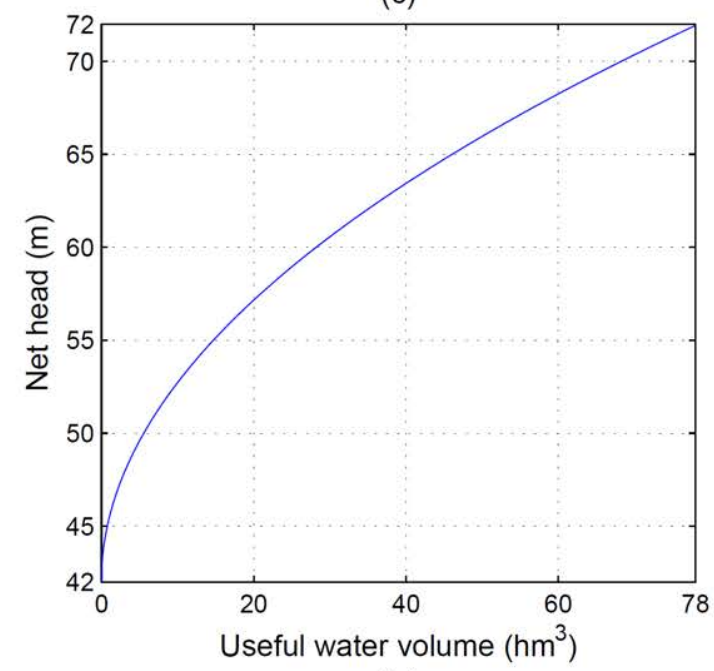

(e)

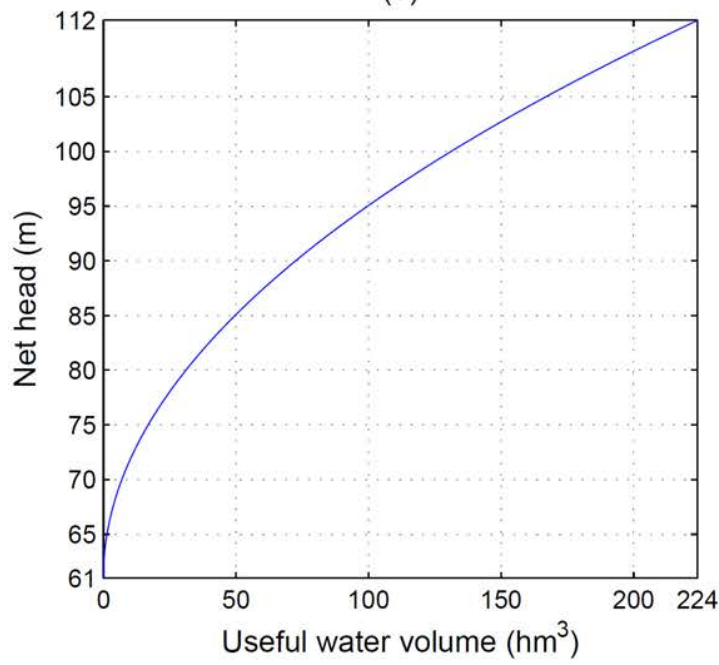

(b)

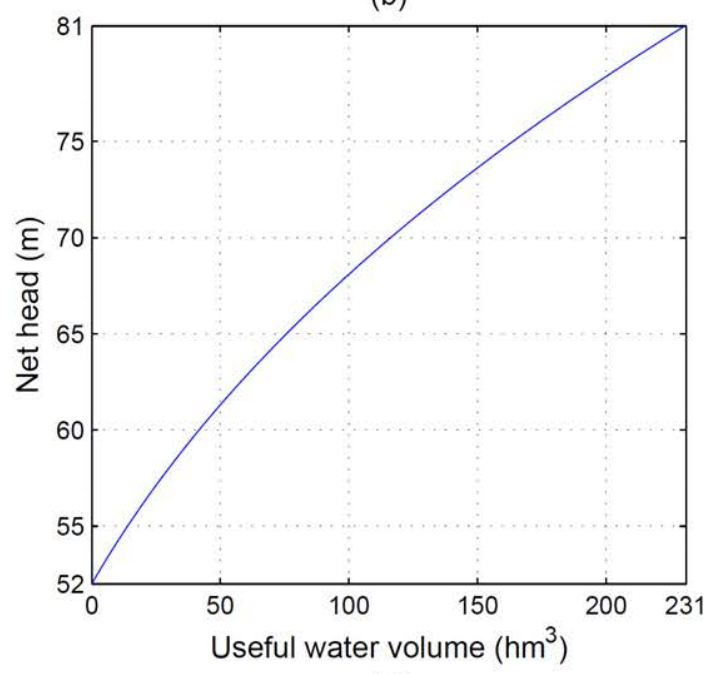

(d)

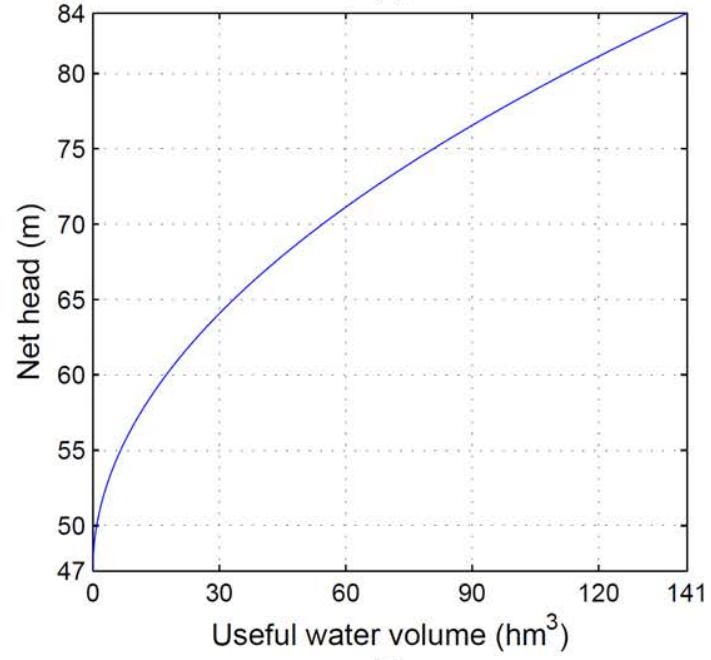

(f)

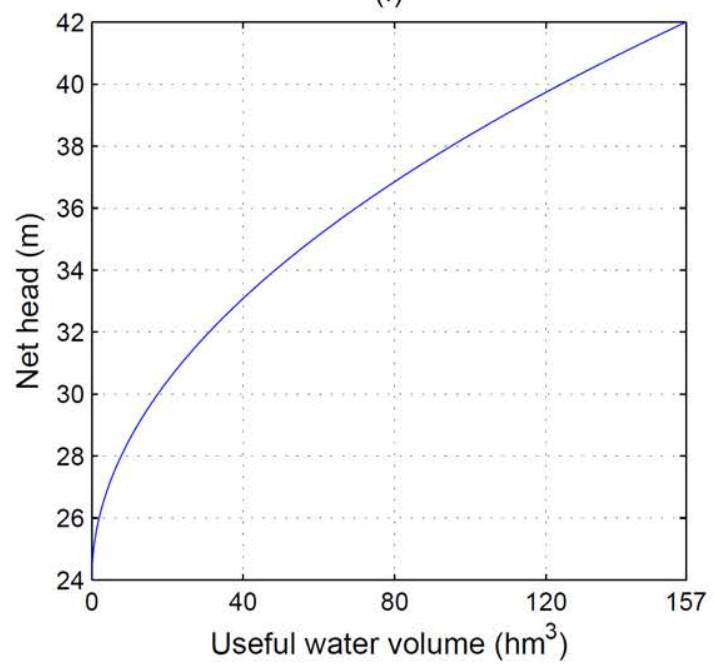

Figure B.18.: Head-storage curves of the plants: (a) I; (b) II; (c) III; (d) IV; (e) V; (f) VI. 
(a)

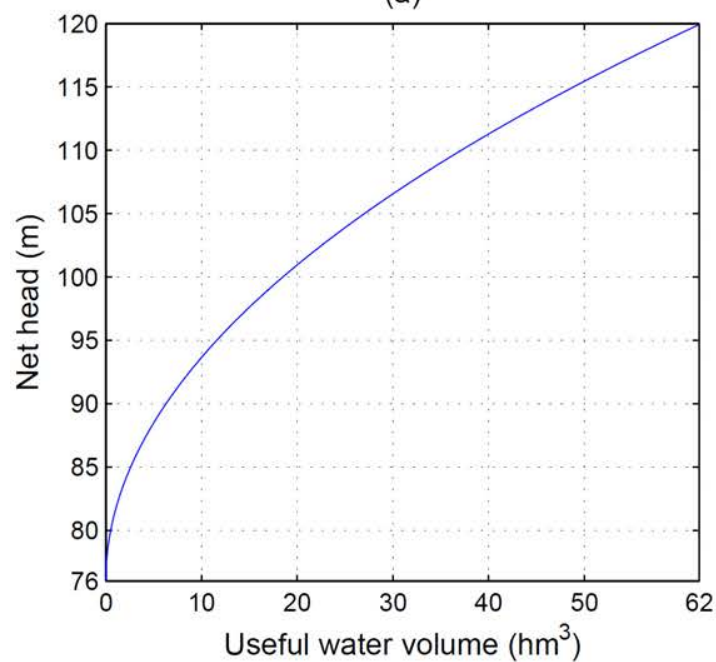

(c)

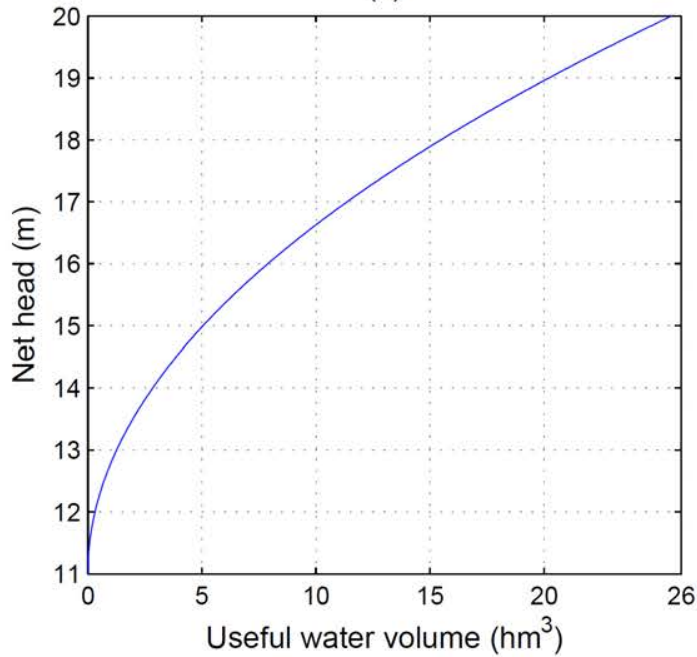

(e)

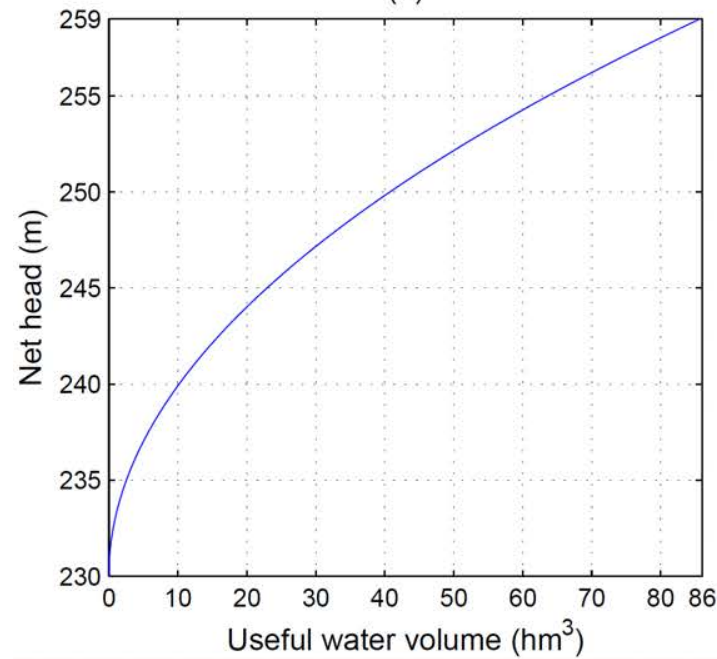

(b)

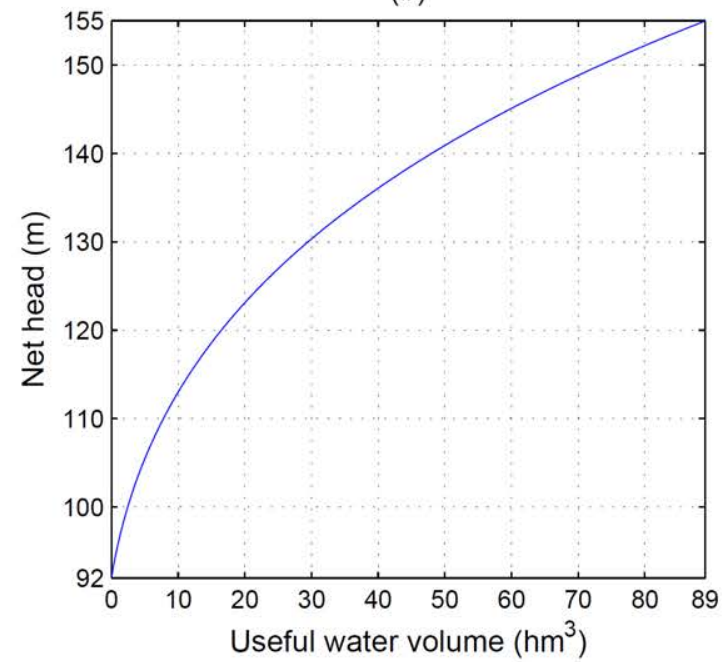

(d)

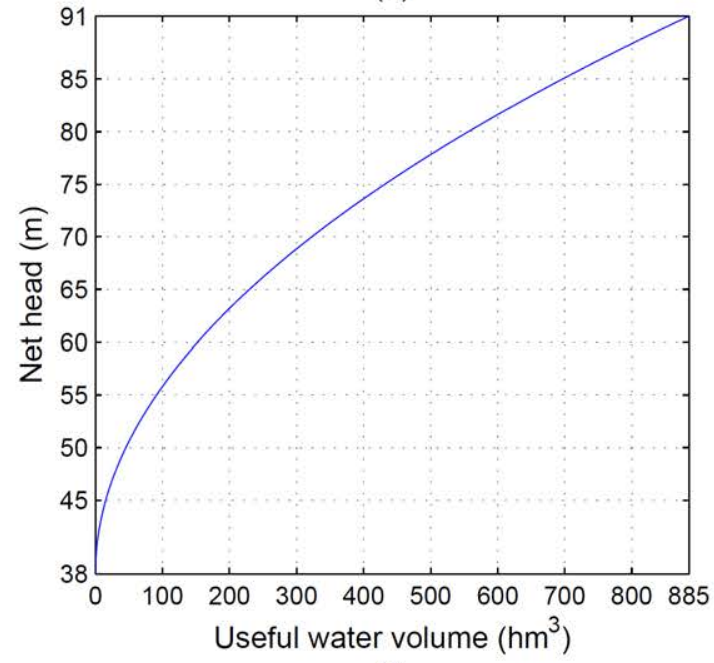

(f)

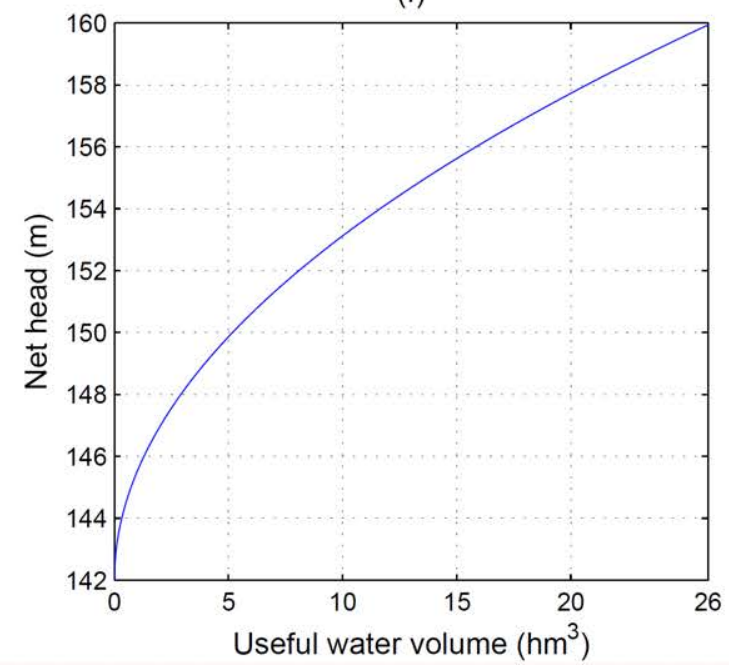

Figure B.19.: Head-storage curves of the plants: (a) VII; (b) VIII; (c) IX; (d) X; (e) A; (f) B. 
(a)

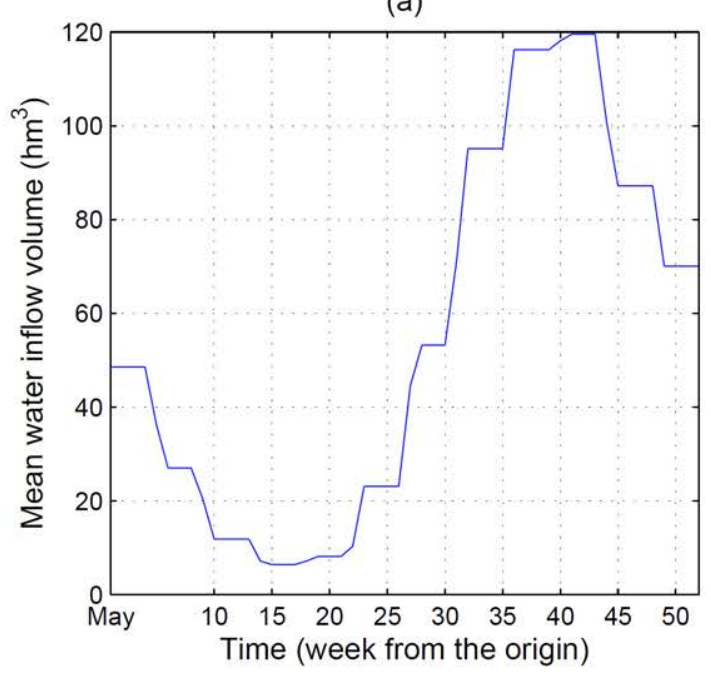

(c)

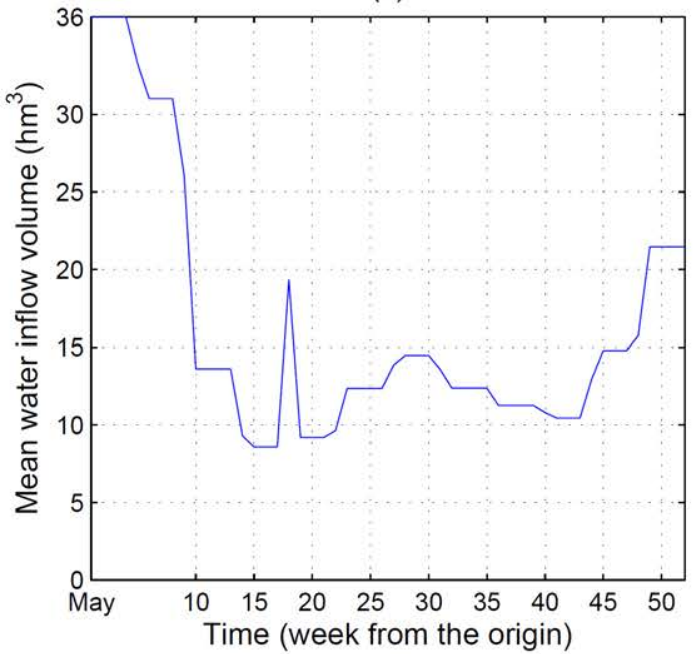

(e)

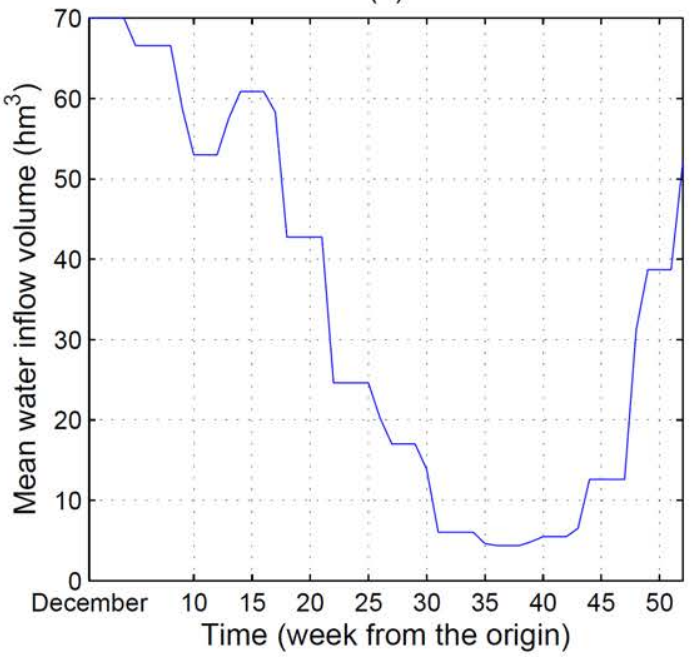

(b)

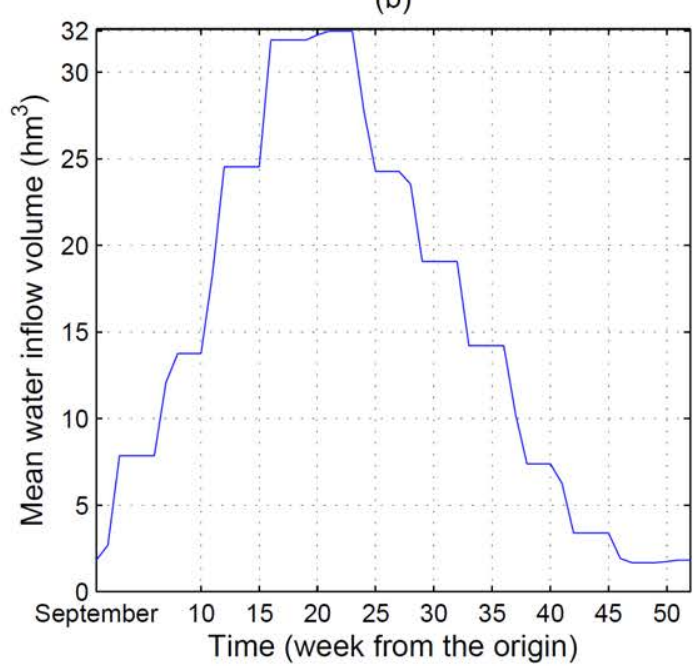

(d)

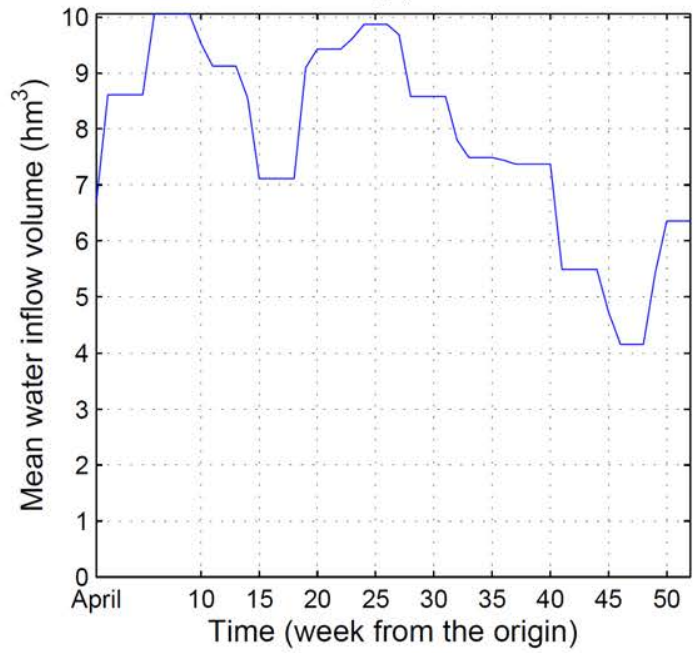

(f)

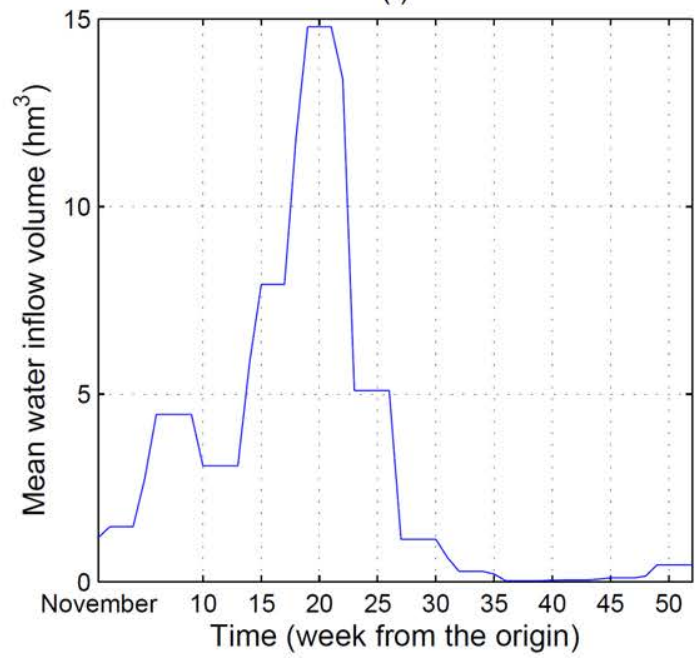

Figure B.20.: Mean water inflow volumes of the plants: (a) I; (b) II; (c) III; (d) IV; (e) V; (f) VI. 
(a)

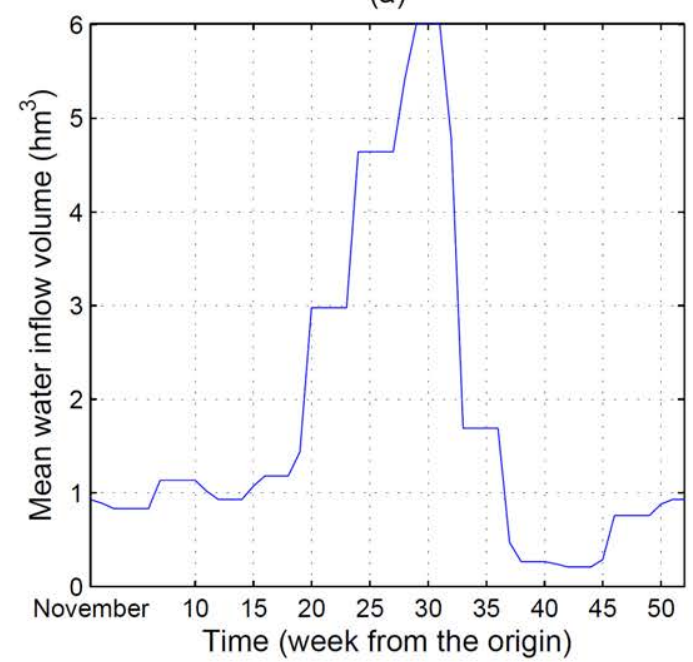

(c)

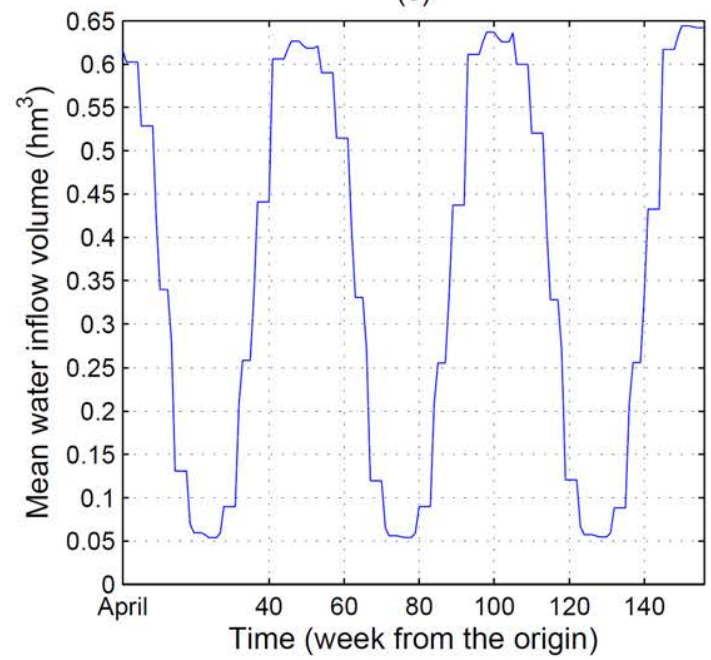

(e)

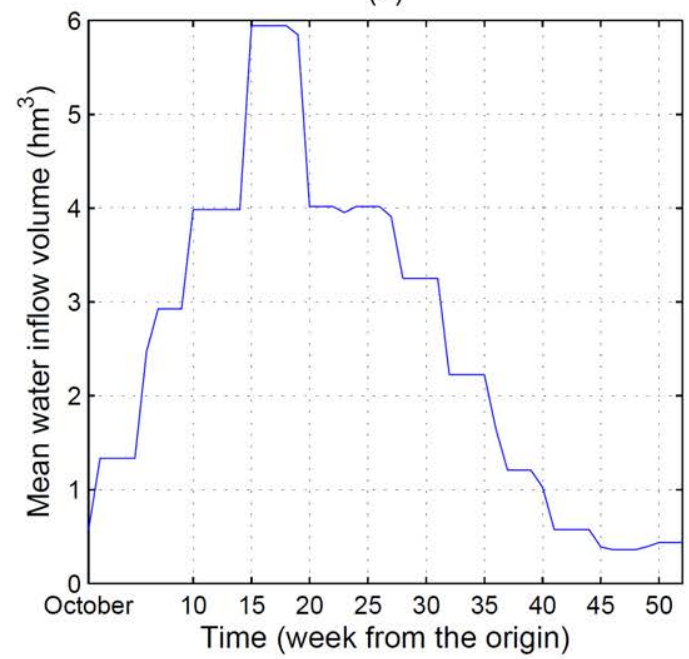

(b)

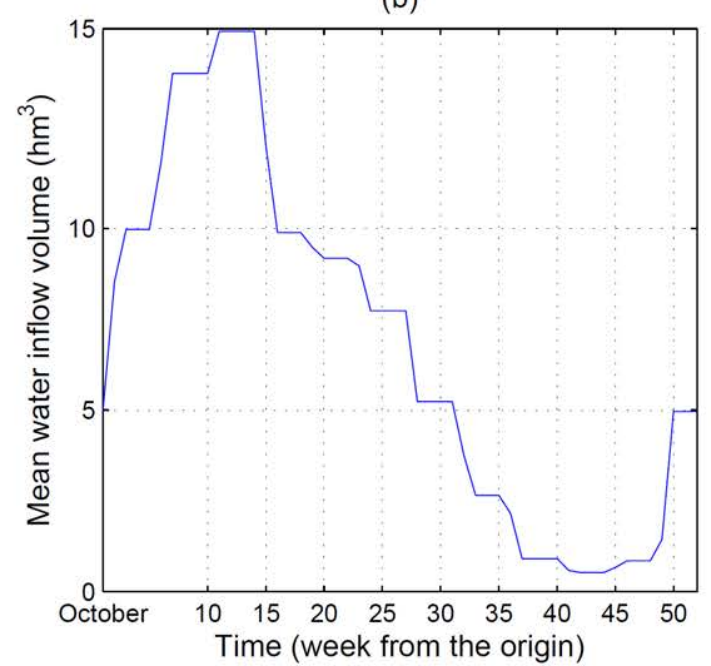

(d)

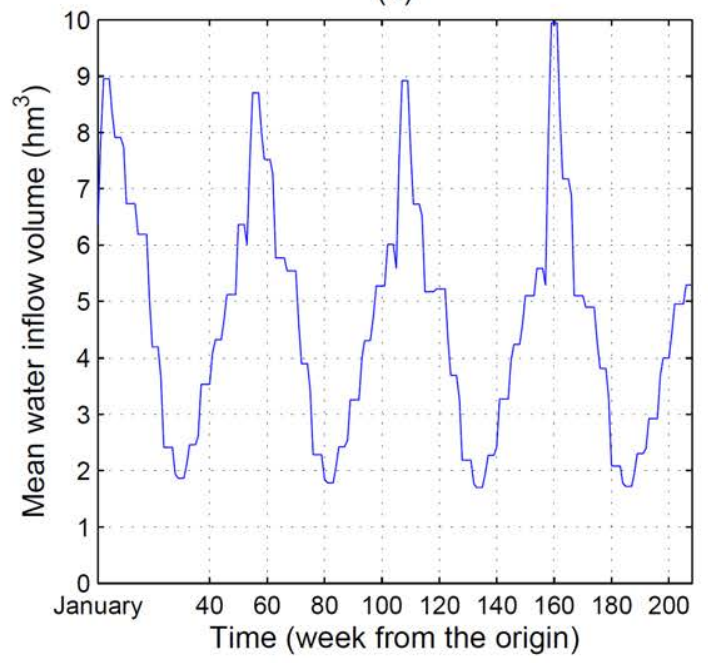

(f)

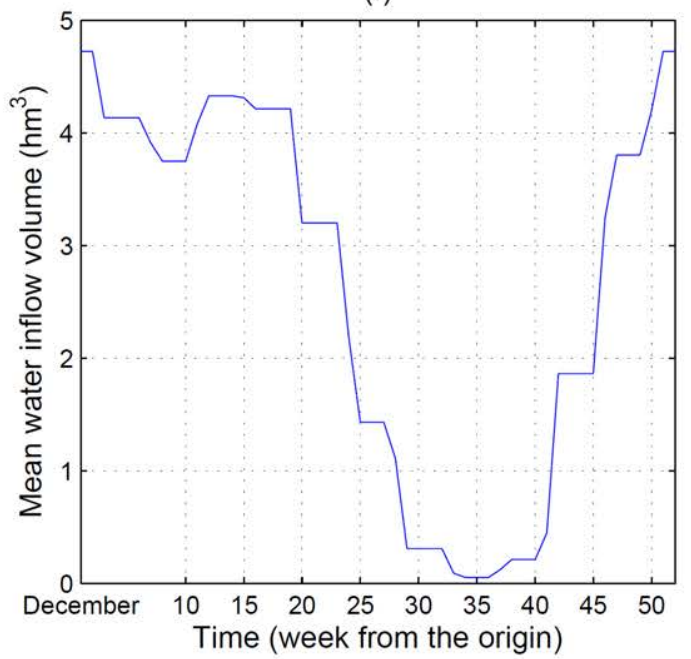

Figure B.21.: Mean water inflow volumes of the plants: (a) VII; (b) VIII; (c) IX; (d) X; (e) A; (f) B. 



\section{References}

Abgottspon, H., Andersson, G., 20-23 May 2012. "Approach of integrating ancillary services into a medium-term hydro optimization". In: 12th symposium of specialists in electric operational and expansion planning. SEPOPE, Rio de Janeiro, Brazil.

ACA, 2015. Water inflows data (from the Catalan Water Agency).

URL http://aca-web.gencat.cat/aca/appmanager/aca/aca?_nfpb=true\&_ pageLabel=P1232354461208201788774

Ahmed, K. M., Petersen, E. H., Arvanitides, N. V., 1965. Optimal operation and control of multi-dam hydroelectric power systems : an application of decision theory. Stanford University, Stanford, USA.

Ahuja, R. K., Magnanti, T. L., Orlin, J. B., 1993. Network flows: theory, algorithms, and applications. Prentice-Hall Incorporated, Upper Saddle River, USA.

Akaike, H., 2-8 September 1971. "Information theory and an extension of the maximum likelihood principle". In: 2nd international symposium on information theory. Akademinai Kiado, Tsahkadsor, Armenia, USSR.

Alemu, E. T., Palmer, R. N., Polebitski, A., Meaker, B., 2011. "Decision support system for optimizing reservoir operations using ensemble streamflow predictions". Journal of Water Resources Planning and Management 137 (1), 72-82.

Allen, R. B., Bridgeman, S. G., 1986. "Dynamic programming in hydropower scheduling". Journal of Water Resources Planning and Management 112 (3), 339-353.

Archibald, T. W., McKinnon, K. I. M., Thomas, L. C., 1997. "An aggregate stochastic dynamic programming model of multireservoir systems". Water Resources Research 33 (2), $333-340$.

Archibald, T. W., McKinnon, K. I. M., Thomas, L. C., 2006. "Modeling the operation of multireservoir systems using decomposition and stochastic dynamic programming". Naval Research Logistics 53 (3), 217-225.

Arroyo, J. M., Conejo, A. J., 2004. "Modeling of start-up and shut-down power trajectories of thermal units". IEEE Transactions on Power Systems 19 (3), 1562-1568. 
Atanassov, E. I., 2003. "A new efficient algorithm for generating the scrambled Sobol'sequence". In: Numerical methods and applications. Springer, Berlin, Germany, pp. 83-90.

Babayev, D. A., Mardanov, S. S., 1994. "Reducing the number of variables in integer and linear programming problems". Computational Optimization and Applications 3 (2), 99109.

Babel, M. S., Nguyen Dinh, C., Mullick, M. R. A., Nanduri, U. V., 2012. "Operation of a hydropower system considering environmental flow requirements: a case study in La Nga River basin, Vietnam". Journal of Hydro-environment Research 6 (1), 63-73.

Basharin, G. P., Langville, A. N., Naumov, V. A., 2004. "The life and work of A.A. Markov". Linear Algebra and its Applications 386, 3-26.

Baslis, C. G., Bakirtzis, A. G., 2011. "Mid-term stochastic scheduling of a price-maker hydro producer with pumped storage". IEEE Transactions on Power Systems 26 (4), 1856-1865.

Bayón, L., Grau, J. M., Ruiz, M. M., Suárez, P. M., 18-22 September 2008. "Influence of the elevation-storage curve in the optimization of hydro-plants". In: 2nd international conference on multidisciplinary design optimization and applications. ASMDO, Gijón, Spain.

Becker, L., Yeh, W. W.-G., 1974. "Optimization of real time operation of a multiple-reservoir system". Water Resources Research 10 (6), 1107-1112.

Bellman, R., 1954. "The theory of dynamic programming". Bulletin of the American Mathematical Society 60 (3), 503-515.

Bellman, R., 1957. Dynamic programming. Princeton University Press, Princeton, USA.

Bellman, R., Dreyfus, S. E., 1962. Applied dynamic programming. Princeton University Press, Princeton, USA.

Bellman, R., Kalaba, R. E., 1965. Dynamic programming and modern control theory. Academic Press, New York, USA.

Belsnes, M. M., Fosso, O. B., 16-18 March 2008. "Competitive optimal hydropower scheduling from strategy to operation". In: 8th IASTED international conference. IASTED, Phuket, Thailand.

Belsnes, M. M., Fosso, O. B., Røynstrand, J., Honve, I., Steinsland, I., Lund, M. W., Sandanger, M. I., Dorn, F., 2013. Building input for SHOP. SINTEF Energi AS, Trondheim, Norway.

Bensalem, A., Miloudi, A., Zouzou, S. E., Mahdad, B., Bouhentala, A., 2007. "Optimal short term hydro scheduling of large power systems with discretized horizon". Journal of Electrical Engineering 58 (4), 214. 
Bernholtz, B., Graham, L., 1960. "Hydrothermal economic scheduling: part I - Solution by incremental dynamic programming". IEEE Transactions on Power Apparatus and Systems 79 (3), 921-929.

Bertsekas, D. P., 1999. Nonlinear programming, 2nd Edition. Vol. 2 of Optimization and computation. Athena Scientific, Belmont, USA.

Bixby, R. E., 2012. "A brief history of linear and mixed-integer programming computation". Documenta Mathematica Extra volume: optimization stories, 107-121.

Bogardi, J. J., Budhakooncharoen, S., Shrestha, D. L., Nandalal, K. D. W., 20-22 July 1988. "Effect of state space and inflow discretization on stochastic dynamic programming based reservoir operation rules and system performance". In: 6th congress Asian and Pacific regional division. IAHR, Kyoto, Japan.

Bolch, G., Greiner, S., de Meer, H., Trivedi, K. S., 2006. Queueing networks and Markov chains: modeling and performance evaluation with computer science applications. John Wiley \& Sons, Inc., New York, USA.

Borghetti, A., D'Ambrosio, C., Lodi, A., Martello, S., 2008. "An MILP approach for shortterm hydro scheduling and unit commitment with head-dependent reservoir". IEEE Transactions on Power Systems 23 (3), 1115-1124.

Boshier, J. F., Manning, G. B., Read, E. G., 1983. "Scheduling releases from New Zealand's hydro reservoirs". Transactions of the Institution of Professional Engineers New Zealand: Electrical/Mechanical/Chemical Engineering Section 10 (2), 33-41.

Box, G. E. P., 1957. "Evolutionary operation: a method for increasing industrial productivity". Applied Statistics 6 (2), 81-101.

BP, 2016. "BP statistical review of world energy June 2016". Tech. rep., British Petroleum. URL http://www.bp.com/content/dam/bp/pdf/energy-economics/ statistical-review-2016/bp-statistical-review-of-world-energy-2016-full-report . pdf

Braga Jr, B. P. F., Yen, W. W.-G., Becker, L., Barros, M. T. L., 1991. "Stochastic optimization of multiple-reservoir-system operation". Journal of Water Resources Planning and Management 117 (4), 471-481.

Bras, R. L., Buchanan, R., Curry, K. C., 1983. "Real time adaptive closed loop control of reservoirs with the High Aswan Dam as a case study". Water Resources Research 19 (1), $33-52$.

Buchanan, L., O‘Connell, A., 2006. "A brief history of decision making". Harvard Business Review 84 (1), 32-41. 
Bunn, D. W., 2004. Modelling prices in competitive electricity markets. Wiley Finance. John Wiley \& Sons, Ltd, Chichester, England.

Carrión, M., Arroyo, J. M., 2006. "A computationally efficient mixed-integer linear formulation for the thermal unit commitment problem". IEEE Transactions on Power Systems $21(3), 1371-1378$.

Carvalho, M. C. A., Pinto, J. M., 2006. "A bilevel decomposition technique for the optimal planning of offshore platforms". Brazilian Journal of Chemical Engineering 23 (1), 67-82.

Castelletti, A., de Rigo, D., Rizzoli, A. E., Soncini-Sessa, R., Weber, E., 2007. "Neurodynamic programming for designing water reservoir network management policies". Control Engineering Practice 15 (8), 1031-1038.

Castelletti, A., Pianosi, F., Soncini-Sessa, R., 2008. "Water reservoir control under economic, social and environmental constraints". Automatica 44 (6), 1595-1607.

Catalão, J. P. S., Mariano, S. J. P. S., Mendes, V. M., Ferreira, L. A. F., 2010. "Nonlinear optimization method for short-term hydro scheduling considering head-dependency". European Transactions on Electrical Power 20 (2), 172-183.

CEDEX, 2013. Water inflows data (from the Centre for Public Works Studies and Experimentation).

URL http://ceh-flumen64.cedex.es/anuarioaforos/default.asp

Cervellera, C., Chen, V. C. P., Wen, A., 2006. "Optimization of a large-scale water reservoir network by stochastic dynamic programming with efficient state space discretization". European Journal of Operational Research 171 (3), 1139-1151.

Chang, G. W., Aganagic, M., Waight, J. G., Medina, J., Burton, T., Reeves, S., Christoforidis, M., 2001. "Experiences with mixed integer linear programming based approaches on short-term hydro scheduling". IEEE Transactions on Power Systems 16 (4), 743-749.

Chen, Z., Forsyth, P. A., 2008. Nonlinear models in mathematical finance: new research trends in option pricing. Vol. 1. Technical University of Berlin, Berlin, Germany, Ch. 9, pp. 275-304.

Cheng, C., Shen, J., Wu, X., Chau, K.-w., 2012. "Short-term hydroscheduling with discrepant objectives using multi-step progressive optimality algorithm". Journal of the American Water Resources Association 48 (3), 464-479.

CHMS, 2010. Maximum values of the environmental constraints (from the Miño-Sil River Basin District Authority).

URL http://www.chminosil.es/es/chms/planificacionhidrologica 
Chow, V. T., Maidment, D. R., Tauxe, G. W., 1975. "Computer time and memory requirements for DP and DDDP in water resource systems analysis". Water Resources Research $11(5), 621-628$.

Colorado State University, 2010. MODSIM 8.1: river basin management decision support system. User manual and documentation. Colorado State University, Fort Collins, USA.

Conejo, A. J., Arroyo, J. M., Contreras, J., Villamor, F. A., 2002. "Self-scheduling of a hydro producer in a pool-based electricity market". IEEE Transactions on Power Systems 17 (4), $1265-1272$.

Cristóbal, M. P., Escudero, L. F., Monge, J. F., 2009. "On stochastic dynamic programming for solving large-scale planning problems under uncertainty". Computers $\&$ Operations Research 36 (8), 2418-2428.

Crombecq, K., Laermans, E., Dhaene, T., 2011. "Efficient space-filling and non-collapsing sequential design strategies for simulation-based modeling". European Journal of Operational Research 214 (3), 683-696.

Cushman, R. M., 1985. "Review of ecological effects of rapidly varying flows downstream from hydroelectric facilities". North American Journal of Fisheries Management 5 (3A), $330-339$.

Cvitanovic, P., Dettmann, C. P., Mainieri, R., Vattay, G., 1999. "Trace formulae for stochastic evolution operators: smooth conjugation method". Nonlinearity 12 (4), 939.

D'Ambrosio, C., Lodi, A., Martello, S., 2010. "Piecewise linear approximation of functions of two variables in MILP models". Operations Research Letters 38 (1), 39-46.

Dantzig, G. B., July 1948. "Linear programming". In: Symposium on numerical methods. Previously invited paper. NBS, Los Angeles, USA.

Dantzig, G. B., 1954. "A comment on Edie's: traffic delays at toll booths". Journal of the Operations Research Society of America 2 (3), 339-341.

Deb, K., 1995. Optimization for engineering design. Algorithms and examples. PHI Learning Pvt. Ltd., New Delhi, India.

Deni, S. M., Jemain, A. A., 4-6 July 2012. "Comparison between mixed probability models and Markov chain models for weekly dry and wet spells in Peninsular Malaysia". In: World congress on engineering. IAENG, London, England.

Dias, B. H., Marcato, A. L. M., Souza, R. C., Soares, M. P., Silva Junior, I. C., Oliveira, E. J. d., Brandi, R., Ramos, T. P., 2010. "Stochastic dynamic programming applied to hydrothermal power systems operation planning based on the convex hull algorithm". Mathematical Problems in Engineering 2010, 390940-390959. 
Diniz, A. L., Souza, T. M., 2014. "Short-term hydrothermal dispatch with river-level and routing constraints". IEEE Transactions on Power Systems PP (99), 1-9.

Doorman, G. L., 2009. Hydro power scheduling. NTNU, Trondheim, Norway.

Doran, D. G., 1975. "An efficient transition definition for discrete state reservoir analysis: the divided interval technique". Water Resources Research 11 (6), 867-873.

Dragoni, W., Valigi, D., 1994. "Contributo alla stima dell evaporazione dalle superfici liquide nell Italia Centrale". Geologica Romana 30, 151-158.

Durán, H., Puech, C., Díaz, J., Sánchez, G., 1985. "Optimal operation of multireservoir systems using an aggregation-decomposition approach". IEEE Transactions on Power Apparatus and Systems 104 (8), 2086-2092.

Eckberg, D. K., 1986. "Cumulative impacts of hydropower development under NEPA". Environmental Law 16, 673-963.

Edwards, B. K., Flaim, S. J., Howitt, R. E., 1999. "Optimal provision of hydroelectric power under environmental and regulatory constraints". Land Economics 75 (2), 267-283.

Egré, D., Milewski, J. C., 2002. "The diversity of hydropower projects". Energy Policy 30 (14), $1225-1230$.

El-Hawary, M. E., Christensen, G. S., 1979. Optimal economic operation of electric power systems. Vol. 26. Academic Press, New York, USA.

EPRI, 2001. "Hydropower Technology Roundup Report: accommodating wear and tear effects on hydroelectric facilities operating to provide ancillary services: TR-113584-V4". Tech. rep., Electric Power Research Institute.

URL http://www.epri.com/abstracts/Pages/ProductAbstract. aspx?ProductId= 000000000001004047

Eschenbach, E. A., Magee, T., Zagona, E., Goranflo, M., Shane, R., 2001. "Goal programming decision support system for multiobjective operation of reservoir systems". Journal of Water Resources Planning and Management 127 (2), 108-120.

ESHA, 2012. "Small hydropower roadmap. Condensed research data for the EU-27". Tech. rep., European Small Hydropower Association.

URL http://streammap.esha.be/fileadmin/documents/Press_Corner_ Publications/SHPRoadmap_FINAL_Public.pdf

EU, 2007. "Water Framework Directive \& hydropower. Key conclusions". Tech. rep., European Union.

URL http://www.ecologic-events.de/hydropower/documents/key_conclusions.pdf 
Eurelectric, 2003. "Ancillary services. Unbundling electricity products. An emerging market". Tech. rep., Union of the Electricity Industry.

URL http://www . eurelectric.org/Download/Download. aspx?DocumentID=13934

Fan, H., Tarun, P. K., Chen, V. C. P., 2013. "Adaptive value function approximation for continuous-state stochastic dynamic programming". Computers $\&$ Operations Research 40 (4), 1076-1084.

Fang, K.-T., Wang, Y., Bentler, P. M., 1994. "Some applications of number-theoretic methods in statistics". Statistical Science 9 (3), 416-428.

Farhat, I. A., El-Hawary, M. E., 2009. "Optimization methods applied for solving the shortterm hydrothermal coordination problem". Electric Power Systems Research 79 (9), 1308 1320.

Fayaed, S. S., El-Shafie, A., Jaafar, O., 2013. "Reservoir-system simulation and optimization techniques". Stochastic Environmental Research and Risk Assessment 27 (7), 1751-1772.

Finardi, E. C., da Silva, E. L., 2005. "Unit commitment of single hydroelectric plant". Electric Power Systems Research 75 (2), 116-123.

Fletcher, R., 2000. Practical methods of optimization, 2nd Edition. John Wiley \& Sons, Ltd, Chichester, England.

Fleten, S. E., Keppo, J., Lumba, H., Weiss, V., 19-22 August 2009. "Derivative price information use in hydroelectric scheduling". In: EFA 36th annual meeting. EFA, Bergen, Norway.

Forsund, F. R., 2007. Hydropower economics. Vol. 112 of International series in operations research and management science. Springer, New York, USA.

Fosso, O. B., Belsnes, M. M., 21-24 November 2004. "Short-term hydro scheduling in a liberalized power system". In: 4th intenational conference on power system technology. IEEE, Singapore, Singapore.

Fosso, O. B., Gjelsvik, A., Haugstad, A., Mo, B., Wangensteen, I., 1999. "Generation scheduling in a deregulated system. The Norwegian case". IEEE Transactions on Power Systems 14 (1), 75-81.

Fourier, J. B. J., 1826. "Solution d une question particuliere du calcul des inégalités". Nouveau Bulletin des Sciences par la Société Philomatique de Paris 99, 100.

Fu, Y., Shahidehpour, M., 2007. "Fast SCUC for large-scale power systems". IEEE Transactions on Power Systems 22 (4), 2144-2151. 
García-González, J., Castro, G. A., 10-13 September 2001. "Short-term hydro scheduling with cascaded and head-dependent reservoirs based on mixed-integer linear programming". In: Power tech conference. IEEE, Porto, Portugal.

Gebrekiros, Y., Doorman, G., Jaehnert, S., Farahmand, H., 6-9 October 2013. "Bidding in the frequency restoration reserves (FRR) market for a hydropower unit". In: 4 th innovative smart grid technologies Europe. IEEE, Copenhagen, Denmark.

Gjelsvik, A., Belsnes, M. M., Haugstad, A., 28 June - 2 July 1999. "An algorithm for stochastic medium-term hydrothermal scheduling under spot price uncertainty". In: 13th power systems computation conference. PSCC, Trondheim, Norway.

Gjelsvik, A., Mo, B., Haugstad, A., 2010. "Long-and medium-term operations planning and stochastic modelling in hydro-dominated power systems based on stochastic dual dynamic programming". In: Handbook of power systems I. Springer, Berlin, Germany, pp. 33-55.

Golnaraghi, M. F., Kuo, B. C., 2003. Automatic control systems. Vol. 4. John Wiley \& Sons, Inc., New York, USA.

Gomory, R. E., 1960. "Outline of an algorithm for integer solutions to linear programs". Bulletin of the American Mathematical Society 28 (1), 30-44.

Goulter, I. C., Tai, F.-K., 1985. "Practical implications in the use of stochastic dynamic programming for reservoir operation". Journal of the American Water Resources Association $21(1), 65-74$.

Grattan-Guinness, I., 1970. "Joseph Fourier's anticipation of linear programming". Operational Research Quarterly 21 (3), 361-364.

Griffith, R. E., Stewart, R. A., 1961. "A nonlinear programming technique for the optimization of continuous processing systems". Management Science 7 (4), 379-392.

Grötschel, M., Jünger, M., Reinelt, G., 1985. "On the acyclic subgraph polytope". Mathematical Programming 33 (1), 28-42.

Grygier, J. C., Stedinger, J. R., 1985. "Algorithms for optimizing hydropower system operation". Water Resources Research 21 (1), 1-10.

Guan, X., Svoboda, A., Li, C., 1999. "Scheduling hydro power systems with restricted operating zones and discharge ramping constraints". IEEE Transactions on Power Systems $14(1), 126-131$.

Guisández, I., Pérez-Díaz, J. I., Wilhelmi, J. R., 2013. "Assessment of the economic impact of environmental constraints on annual hydropower plant operation". Energy Policy 61, $1332-1343$. 
Guisández, I., Pérez-Díaz, J. I., Wilhelmi, J. R., 8-10 April 2014. "Effects of the maximum flow ramping rates on the long-term operation decisions of a hydropower plant". In: International conference on renewable energies and power quality. ICREPQ, Córdoba, Spain.

Guisández, I., Pérez-Díaz, J. I., Wilhelmi, J. R., 17-18 September 2015. "Influence of the maximum flow ramping rates on the water value". In: 5th international workshop on hydro scheduling in competitive electricity markets. IWHSCEM, Trondheim, Norway.

Guisández, I., Pérez-Díaz, J. I., Wilhelmi, J. R., 2016a. "Approximate formulae for the assessment of the long-term economic impact of environmental constraints on hydropeaking". Energy 112, 629-641.

Guisández, I., Pérez-Díaz, J. I., Wilhelmi, J. R., 2016b. "Influence of the maximum flow ramping rates on the water value". Energy Procedia 87, 100-107.

Guisández, I., Pérez-Díaz, J. I., Wilhelmi, J. R., 2016c. "The influence of environmental constraints on the water value". Energies 9 (6).

Guise, J. W. B., Flinn, J. C., 1970. "The allocation and pricing of water in a river basin". American Journal of Agricultural Economics 52 (3), 411-421.

Habibollahzadeh, H., Bubenko, J. A., 1986. "Application of decomposition techniques to short-term operation planning of hydrothermal power system". IEEE Transactions on Power Systems 1 (1), 41-47.

Halton, J. H., 1960. "On the efficiency of certain quasi-random sequences of points in evaluating multi-dimensional integrals". Numerische Mathematik 2 (1), 84-90.

Hamilton, H., 1767. Philosophical essays: on the following subjects: I. On the ascent of vapours, the formation of clouds, rain and dew, and on several other phoenomena of air and water, II. Observations and conjectures on the nature of the aurora borealis, and the tails of comets, III. On the principles of mechanicks, 2nd Edition. John Nourse, London, England.

Hammersley, J. M., 1960. "Monte Carlo methods for solving multivariable problems". Annals of the New York Academy of Sciences 86 (3), 844-874.

Harbeck, G. E., 1962. "A practical field technique for measuring reservoir evaporation utilizing mass-transfer theory". U.S. Geological Survey 272-E, 101-105.

Harpman, D. A., 1999. "Assessing the short-run economic cost of environmental constraints on hydropower operations at Glen Canyon Dam". Land Economics 75 (3), 390-401.

Hatch Ltd., 2013. Vista DSS. Statement of qualifications for power and water optimization (PWO). Hatch Ltd., Mississauga, Canada. 
Helseth, A., Gjelsvik, A., Mo, B., Linnet, Ú., 2013. "A model for optimal scheduling of hydro thermal systems including pumped-storage and wind power". IET Generation, Transmission $\&$ Distribution 7 (12), 1426-1434.

Holland, J. H., 1975. Adaptation in natural and artificial systems. University of Michigan Press, Ann Arbor, USA.

Howard, C. D. D., 24-27 October 2006. "Hydroelectric system operations optimization". In: Great Wall world renewable energy forum and exhibition. CWEA, Beijing, China.

Howard, R. A., 1960. Dynamic programming and Markov processes. The M.I.T. Press, Cambridge, USA.

Howson, H. R., Sancho, N. G. F., 1975. "A new algorithm for the solution of multi-state dynamic programming problems". Mathematical programming 8 (1), 104-116.

Iliadis, N. A., Tilmant, A., Chabar, R., Granville, S., Pereira, M., 17-23 September 2008. "Optimal operation of hydro-dominated multireservoir systems in deregulated electricity markets". In: International conference for reservoir operation and river management. ICROM, Guanghzou and Three Gorges, China.

Illés, T., Terlaky, T., 2002. "Pivot versus interior point methods: pros and cons". European Journal of Operational Research 140 (2), 170-190.

INE, 1960. Temperature data (from the Spanish Statistical Office).

URL http://www.ine.es/inebaseweb/pdfDispacher.do; jsessionid= 09739D168C9F3EF056383E89BACE7A07 . inebaseweb01?td=183760

Jensen, S. G., Skytte, K., 2003. "Simultaneous attainment of energy goals by means of green certificates and emission permits". Energy Policy 31 (1), 63-71.

Jimoh, O. D., Webster, P., 1996. "The optimum order of a Markov chain model for daily rainfall in Nigeria". Journal of Hydrology 185 (1), 45-69.

Johnson, S. A., Stedinger, J. R., Shoemaker, C. A., Li, Y., Tejada-Guibert, J. A., 1993. "Numerical solution of continuous-state dynamic programs using linear and spline interpolation". Operations Research 41 (3), 484-500.

Juday, D. G., Brummer, J. E., Smith, D. H., 2011. "Use of alternative temperature expressions with Blaney-Criddle". Journal of Irrigation and Drainage Engineering 137 (9), 573-584.

Kall, P., Mayer, J., 2011. Stochastic linear programming. Models, theory, and computation. Vol. 156 of International Series in Operations Research \& Management Science. Springer, New York, USA.

Kapelan, Z., Savic, D. A., Walters, G. A., 2005. "Decision-support tools for sustainable urban development". Proceedings of the ICE-Engineering Sustainability 158 (3), 135-142. 
Karamouz, M., Houck, M. H., 1987. "Comparison of stochastic and deterministic dynamic programming for reservoir operating rule generation". Journal of the American Water Resources Association 23 (1), 1-9.

Karamouz, M., Zahraie, B., Araghinejad, S., 2005. "Decision support system for monthly operation of hydropower reservoirs: a case study". Journal of Computing in Civil Engineering 19 (2), 194-207.

Karmarkar, N., 30 April - 2 May 1984. "A new polynomial-time algorithm for linear programming". In: 16th annual symposium on theory of computing. ACM, Washington, USA.

Karush, W., 1939. Minima of functions of several variables with inequalities as side constraints. Ph.D. thesis, The University of Chicago, Chicago, USA.

Katz, R. W., 1981. "On some criteria for estimating the order of a Markov chain". Technometrics 23 (3), 243-249.

Keckler, W. G., Larson, R. E., 1968. "Dynamic programming applications to water resource system operation and planning". Journal of Mathematical Analysis and Applications 24 (1), 80-109.

Kelman, R., Barroso, L. A. N., Pereira, M. V. F., 2001. "Market power assessment and mitigation in hydrothermal systems". IEEE Transactions on Power Systems 16 (3), 354359 .

Kemeny, J. G., Snell, J. L., 1960. Finite Markov chains. Van Nostrand Company, Princeton, USA.

Keppo, J., 2002. "Optimality with hydropower system". IEEE Transactions on Power Systems $17(3), 583-589$.

Kim, Y. O., Palmer, R. N., 1997. "Value of seasonal flow forecasts in Bayesian stochastic programming". Journal of Water Resources Planning and Management 123 (6), 327-335.

Kirchhoff, G. R., 1845. "Ueber den Durchgang eines elektrischen Stromes durch eine Ebene, insbesondere durch eine kreisförmige". Annalen der Physik 140 (4), 497-514.

Kirkham, H., Nightingale, D., Koerner, T., 1981. "Energy management system design with dispersed storage and generation". IEEE Transactions on Power Apparatus and Systems 100 (7), 3432-3441.

Kishen, K., 1942. "On Latin and hyper-Graeco-Latin cubes and hyper-cubes". Current Science $11(3), 98-99$.

Klemeš, V., 1977. "Discrete representation of storage for stochastic reservoir optimization". Water Resources Research 13 (1), 149-158. 
Knittel, C. R., Roberts, M. R., 2005. "An empirical examination of restructured electricity prices". Energy Economics 27 (5), 791-817.

Kolmogoroff, A., 1936. "Zur theorie der markoffschen ketten". Mathematische Annalen 112 (1), $155-160$.

Koreneff, G., Seppala, A., Lehtonen, M., Kekkonen, V., Laitinen, E., Hakli, J., Antila, E., 3-5 March 1998. "Electricity spot price forecasting as a part of energy management in deregulated power market". In: International conference on energy management and power delivery. IEEE, Singapore.

Kosnik, L.-R. D., 2010. "Balancing environmental protection and energy production in the Federal hydropower licensing process". Land Economics 86 (3), 444-466.

Kotchen, M. J., Moore, M. R., Lupi, F., Rutherford, E. S., 2006. "Environmental constraints on hydropower: an ex post benefit-cost analysis of dam relicensing in Michigan". Land Economics 82 (3), 384-403.

Koutsoyiannis, D., Efstratiadis, A., Karavokiros, G., 2002. "A decision support tool for the management of multi-reservoir systems". Journal of the American Water Resources Association 38 (4), 945-958.

Kuhn, H. W., Tucker, A. W., 31 July - 12 August 1950. "Nonlinear programming". In: 2nd Berkeley symposium on mathematical statistics and probability. University of California, Berkeley, USA.

Kumar, N., Baliarsingh, F., 2003. "Folded dynamic programming for optimal operation of multireservoir system". Water Resources Management 17 (5), 337-353.

Labadie, J. W., 2004. "Optimal operation of multireservoir systems: state-of-the-art review". Journal of Water Resources Planning and Management 130 (2), 93-111.

Land, A. H., Doig, A. G., 1960. "An automatic method of solving discrete programming problems". Econometrica 28 (3), 497-520.

Larson, R., 1967. "A survey of dynamic programming computational procedures". IEEE Transactions on Automatic Control 12 (6), 767-774.

Lehner, B., Czisch, G., Vassolo, S., 2005. "The impact of global change on the hydropower potential of Europe: a model-based analysis". Energy Policy 33 (7), 839-855.

Li, T., Shahidehpour, M., 2005. "Price-based unit commitment: a case of Lagrangian relaxation versus mixed integer programming". IEEE Transactions on Power Systems 20 (4), $2015-2025$. 
Lima, R. M., Grossmann, I. E., 2011. "Computational advances in solving mixed integer linear programming problems". In: Chemical engineering greetings to prof. Sauro Pierucci on occasion of his 65th birthday. The Italian Association of Chemical Engineering, Milano, Italy, pp. 151-160.

Little, J. D. C., 1955. "The use of storage water in a hydroelectric system". Operations Research 3 (2), 187-197.

Liu, X., Guo, S., Liu, P., Chen, L., Li, X., 2011. "Deriving optimal refill rules for multi-purpose reservoir operation". Water Resources Management 25 (2), 431-448.

Löhndorf, N., Wozabal, D., Minner, S., 2013. "Optimizing trading decisions for hydro storage systems using approximate dual dynamic programming". Operations Research 61 (4), 810823.

Loucks, D. P., Stedinger, J. R., Haith, D. A., 1981. Water resource systems planning and analysis. Prentice-Hall Incorporated, Englewood Cliffs, USA.

Loucks, D. P., van Beek, E., 2005. Water resources systems planning and management. An introduction to methods, models and applications. UNESCO, Paris, France.

Lowry, W. P., Guthrie, D., 1968. "Markov chains of order greater than one". Monthly Weather Review 96 (11), 798-801.

Luenberger, D. G., 2003. Linear and nonlinear programming. Springer, Boston, USA.

MacCallum, R. C., Widaman, K. F., Zhang, S., Hong, S., 1999. "Sample size in factor analysis". Psychological Methods 4 (1), 84-99.

Mariño, M. A., Mohammadi, B., 1983. "Reservoir operation by linear and dynamic programming". Journal of Water Resources Planning and Management 109 (4), 303-319.

Markov, A. A., 1906. "Rasprostranenie zakona bol shih chisel na velichiny, zavisyaschie drug ot druga". Izvestiya Fiziko-matematicheskogo obschestva pri Kazanskom universitete 15 (2), $135-156$.

Markowitz, H. M., Manne, A. S., 1957. "On the solution of discrete programming problems". Econometrica 25 (1), 84-110.

Martin, Q. W., 1983. "Optimal operation of multiple reservoir systems". Journal of Water Resources Planning and Management 109 (1), 58-74.

Martino, J. P., 1993. Technological forecasting for decision making, 3rd Edition. McGraw-Hill, Inc., New York, USA.

Maruzewski, P., Rogeaux, C., Laurier, P., 19-23 August 2012. "Unit-sizing of hydro power plant". In: IOP conference series: earth and environmental science. IAHR, Beijing, China. 
Mazliak, L., 2007. "On the exchanges between Wolfgang Doeblin and Bohuslav Hostinsky". Revue d'histoire des mathématiques 13 (1), 155-180.

Meyn, S. S. P., Tweedie, R. L., 1993. Markov chains and stochastic stability. Springer-Verlag, London, England.

Mitchell, J. E., 2002. Handbook of applied optimization. Vol. 1. Oxford University Press, Oxford, England, Ch. 3.3, pp. 65-77.

Mo, B., Gjelsvik, A., Grundt, A., Kåresen, K., 10-13 September 2001. "Optimisation of hydropower operation in a liberalised market with focus on price modelling". In: Power tech conference. IEEE, Porto, Portugal.

Mo, B., Haugstad, A., Fosso, O. B., 30 June - 2 July 1997. "Integrating Long and Short-Term Modes for Hydro Scheduling". In: 3rd international conference on hydropower. NTNU, Trondheim, Norway.

Moran, P. A. P., 1959. The theory of storage. Metheun Publications, London, England.

Morgan, H. D., Doran, W. E., Mair, J., Edginton, R. H., Addison, H., Thornton, J. H., 1957. "Discussion on hydraulics paper no. 16: control of flow by gates and valves". ICE Proceedings 7 (3), 569-574.

Nandalal, K. D. W., Bogardi, J. J., 2007. Dynamic programming based operation of reservoirs. Applicability and limits. Cambridge University Press, Cambridge, England.

Niederreiter, H., 1992. Quasi-Monte Carlo methods. John Wiley \& Sons, Inc., New York, USA.

Nilsson, O., Sjelvgren, D., 1997a. "Hydro unit start-up costs and their impact on the short term scheduling strategies of Swedish power producers". IEEE Transactions on Power Systems 12 (1), 38-44.

Nilsson, O., Sjelvgren, D., 1997b. "Variable splitting applied to modelling of start-up costs in short term hydro generation scheduling". IEEE Transactions on Power Systems 12 (2), $770-775$.

Niu, S., Insley, M., 2010. "On the economics of ramping rate restrictions at hydro power plants: balancing profitability and environmental costs". Working paper, University of Waterloo.

URL affinity.uwaterloo.ca/ minsley/ramping $\% 20$ rate $\% 20$ paper $\% 20 \mathrm{Jan} \% 203 \%$ 202011.pdf

Niu, S., Insley, M., 2013. "An options pricing approach to ramping rate restrictions at hydro power plants". Working paper, University of Waterloo.

URL economics.ualberta.ca/en/EventsandSeminars/EMEE2013/ /media/economics/ EventsAndS/EMEE-2013/AP-Niu-Insley.pdf 
Nocedal, J., Wright, S. J., 2006. Numerical optimization, 2nd Edition. Operations Research and Financial Engineering. Springer, New York, USA.

Nogales, F. J., Contreras, J., Conejo, A. J., Espínola, R., 2002. "Forecasting next-day electricity prices by time series models". IEEE Transactions on Power Systems 17 (2), 342-348.

Norris, J. R., 1998. Markov chains. No. 2008. Cambridge University Press, Cambridge, England.

Olivares, M. A., 2008. Optimal hydropower reservoir operation with environmental requirements. Ph.D. thesis, The University of California, Davis, USA.

Olivares, M. A., Haas, J., Palma-Behnke, R., Benavides, C., 2015. "A framework to identify Pareto-efficient subdaily environmental flow constraints on hydropower reservoirs using a grid-wide power dispatch model". Water Resources Research 51 (5), 3664-3680.

Olivares, M. A., Lund, J. R., 2011. "Representing energy price variability in long-and mediumterm hydropower optimization". Journal of Water Resources Planning and Management 138 (6), 606-613.

OMIE, 2011. Day-ahead market data (from the Iberian Electricity Market Operator).

URL http://www.omel.es/files/flash/ResultadosMercado.swf

O’Neil, K., Bernstein, A., Steinschneider, S., Polebitski, A., Palmer, R., 20-22 May 2013. "Modeling the impact of climate change on hydropower operations in the Connecticut River basin". In: World environmental and water resources congress. ASCE, Cincinnati, USA.

Ortuño, M. F., Conejero, W., Moreno, F., Moriana, A., Intrigliolo, D. S., Biel, C., Mellisho, C. D., Pérez-Pastor, A., Domingo, R., Ruiz-Sánchez, M. C., Casadeus, J., Bonany, J., Torrecillas, A., 2010. "Could trunk diameter sensors be used in woody crops for irrigation scheduling? A review of current knowledge and future perspectives". Agricultural Water Management 97 (1), 1-11.

Padhy, N. P., 2004. "Unit commitment-a bibliographical survey". IEEE Transactions on Power Systems 19 (2), 1196-1205.

Paredes-Arquiola, J., Martinez-Capel, F., Solera, A., Aguilella, V., 2011. "Implementing environmental flows in complex water resources systems-case study: the Duero River basin, Spain". River Research and Applications 29 (4), 451-468.

Patten, D. T., Harpman, D. A., Voita, M. I., Randle, T. J., 2001. "A managed flood on the Colorado River: background, objectives, design, and implementation". Ecological Applications 11 (3), 635-643. 
Pearson, K., 1901. "On lines and planes of closest fit to systems of points in space". The London, Edinburgh, and Dublin Philosophical Magazine and Journal of Science 2 (11), $559-572$.

Pelc, R., Fujita, R. M., 2002. "Renewable energy from the ocean". Marine Policy 26 (6), $471-479$.

Pereira, M., 22-25 July 1985. "Optimal scheduling of hydrothermal systems-an overview". In: Symposium on planning and operation of electric energy systems. IFAC, Rio de Janeiro, Brazil.

Perekhodtsev, D., Lave, L., 2005. "Efficient bidding for hydro power plants in markets for energy and ancillary services". Working paper, Massachusetts Institute of Technology.

URL http://scholar.google.es/scholar?start=10\&q=\%22ancillary+services\%22+ hydropower\&hl=es\&as_sdt $=1,5 \& a s_{-}$vis $=1$

Perera, K. K. Y. W., 1969. A study of power system economic operation. Ph.D. thesis, The University of British Columbia, Vancouver, Canada.

Pérez-Díaz, J. I., Millán, R., García, D., Guisández, I., Wilhelmi, J. R., 2012. "Contribution of re-regulation reservoirs considering pumping capability to environmentally friendly hydropower operation". Energy 48 (1), 144-152.

Pérez-Díaz, J. I., Wilhelmi, J. R., 2010. "Assessment of the economic impact of environmental constraints on short-term hydropower plant operation". Energy Policy 38 (12), 7960-7970.

Pérez-Díaz, J. I., Wilhelmi, J. R., Arévalo, L. A., 2010. "Optimal short-term operation schedule of a hydropower plant in a competitive electricity market". Energy Conversion and Management 51 (12), 2955-2966.

Pérez González, M., Sanz Donaire, J., 2001. "Aspectos geográficos de las distribuciones estadísticas ajustadas a las series temporales rellenadas de totales anuales de precipitación española". Nimbus 7-8, 135-159.

Perrin, F., 1971. "Recent advances in energy systems". Annals of the New York Academy of Sciences 184 (1), 242-251.

Petreski, Z., 6-9 July 1999. "Waterpower optimization software: WOPRO 4.0-ALLOCATE 2.0". In: Waterpower conference 1999. ASCE, Las Vegas, USA.

Philbrick, C. R., Kitanidis, P. K., 1999. "Limitations of deterministic optimization applied to reservoir operations". Journal of Water Resources Planning and Management 125 (3), $135-142$.

Philbrick, C. R., Kitanidis, P. K., 2001. "Improved dynamic programming methods for optimal control of lumped-parameter stochastic systems". Operations Research 49 (3), 398-412. 
Philpott, A. B., Dallagi, A., Gallet, E., 2013. "On cutting plane algorithms and dynamic programming for hydroelectricity generation". In: Handbook of risk management in energy production and trading. Springer, Berlin, Germany, pp. 105-127.

Piekutowski, M. R., Litwinowicz, T., Frowd, R., 4-7 May 1993. "Optimal short-term scheduling for a large-scale cascaded hydro system". In: Power industry computer application conference. IEEE, Phoenix, USA.

Pousinho, H. M. I., Mendes, V. M. F., Catalão, J. P. S., 2012. "Scheduling of a hydro producer considering head-dependency, price scenarios and risk-aversion". Energy Conversion and Management 56, 96-103.

Preda, V., Balcau, C., 2008. "Homogeneous stationary multiple Markov chains with maximum Iosifescu-Theodorescu entropy". Revue Roumaine de Mathematiques Pures et Appliquees $53(1), 55-62$.

Pritchard, G., Philpott, A. B., Neame, P. J., 2005. "Hydroelectric reservoir optimization in a pool market". Mathematical Programming 103 (3), 445-461.

Raabe, J., 1985. Hydro power: the design, use, and function of hydromechanical, hydraulic, and electrical equipment. VDI-Verlag, Dusserldorf, Germany.

Rani, D., Moreira, M. M., 2010. "Simulation-optimization modeling: a survey and potential application in reservoir systems operation". Water Resources Management 24 (6), 11071138.

Ratnayake, U., Harboe, R., 2007. "Deterministic and stochastic optimization of a reservoir system". Water International 32 (1), 155-162.

Rauscher, B., Olason, T., Welt, F., Bridgeman, S. G., 29 July - 2 August 2002. "Environmental compliance and optimum hydroelectric system dispatch". In: HydroVision 2002 conference. HCI Publications, Inc., Portland, USA.

Reneses, J., Barquín, J., García-González, J., Centeno, E., 2015. "Water value in electricity markets". International Transactions on Electrical Energy Systems.

Rheinheimer, D. E., Yarnell, S. M., Viers, J. H., 2013. "Hydropower costs of environmental flows and climate warming in California's Upper Yuba River watershed". River Research and Applications 29 (10), 1291-1305.

Robitaille, A., Audette, L., Tinker, D., 17-20 October 2005. "Benefits in optimizing hydro plant operations while meeting sophisticated environmental requirements". In: Hydro 2005. Hydropower \& Dams and NetWork Events Ltd, Villach, Austria.

Rosenberry, D. O., Winter, T. C., Buso, D. C., Likens, G. E., 2007. "Comparison of 15 evaporation methods applied to a small mountain lake in the Northeastern USA". Journal of Hydrology 340 (3), 149-166. 
Rossi, G. B., 2012. "Toward an interdisciplinary probabilistic theory of measurement". IEEE Transactions on Instrumentation and Measurement 61 (8), 2095-2106.

Saad, M., Turgeon, A., 1988. "Application of principal component analysis to long-term reservoir management". Water Resources Research 24 (7), 907-912.

Sanguedo Baptista, R., De Aquino Galeano Massera Da Hora, M., 2013. "Estimated ecological flow of the Preto River by the wetted perimeter method". Global Journal of Researches in Engineering 13 (7).

Sauter, T., Lobashov, M., 2011. "End-to-end communication architecture for smart grids". IEEE Transactions on Industrial Electronics 58 (4), 1218-1228.

Schwarz, G., 1978. "Estimating the dimension of a model". The Annals of Statistics 6 (2), 461-464.

Schweppe, F. C., Tabors, R. D., Kirtley, J. L., Outhred, H. R., Pickel, F. H., Cox, A. J., 1980. "Homeostatic utility control". IEEE Transactions on Power Apparatus and Systems PAS-99 (3), 1151-1163.

Shafer, J. M., Labadie, J. W., 1978. "Synthesis and calibration of a river basin water management model". Tech. rep., Office of Water Research and Technology.

URL http://cwi.colostate.edu/publications/cr/89.pdf

Simon, H. A., 1956. "Dynamic programming under uncertainty with a quadratic criterion function". Econometrica 24 (1), 74-81.

Siu, T. K., Nash, G. A., Shawwash, Z. K., 2001. "A practical hydro, dynamic unit commitment and loading model". IEEE Transactions on Power Systems 16 (2), 301-306.

Smeers, Y., 2008. "Study on the general design of electricity market mechanisms close to real time". Tech. rep., Commission for Electricity and Gas Regulation.

URL http://www.creg.info/pdf/Etudes/F810UK.pdf

Smith, B. T., Jager, H. I., March, P., 23-26 July 2007. "Prospects for combining energy and environmental objectives in hydropower optimization". In: Waterpower 15th conference and exhibition. HCI Publications, Inc., Chattanooga, USA.

Soares, S., Carneiro, A. A. F. M., 1991. "Optimal operation of reservoirs for electric generation". IEEE Transactions on Power Delivery 6 (3), 1101-1107.

Sobol, I. M., 1967. "O raspredelenii tochek v kube i priblizhennom vychislenii integralov". ZH. vychisl. matem. i matem. fiz. 7 (4), 86-112.

Solow, A. R., Smith, W. K., 2006. "Using Markov chain successional models backwards". Journal of Applied Ecology 43 (1), 185-188. 
Stage, S., Larsson, Y., 1961. "Incremental cost of water power". Power Apparatus and Systems, Part III. Transactions of the American Institute of Electrical Engineers 80 (3), 361-364.

Stedinger, J. R., Faber, B. A., Lamontagne, J. R., 19-23 May 2013. "Developments in stochastic dynamic programming for reservoir operation optimization". In: World environmental and water resources congress 2013: showcasing the future. ASCE, Cincinnati, USA.

Stedinger, J. R., Sule, B. F., Loucks, D. P., 1984. "Stochastic dynamic programming models for reservoir operation optimization". Water Resources Research 20 (11), 1499-1505.

Steinschneider, S., Bernstein, A., Palmer, R., Polebitski, A., 2014. "Reservoir management optimization for basin-wide ecological restoration in the Connecticut River". Journal of Water Resources Planning and Management 140 (9).

Stillwater Sciences, Whittaker, D., Shelby, B., Toepel, K. A., Oetting, A. C., 2006. "Scientific Approaches for Evaluating Hydroelectric Project Effects". Tech. rep., Hydropower Reform Coalition.

URL http://www.hydroreform.org/sites/default/files/HRC-\%20Scientific\% 20 approaches $\% 20$ for $\% 20$ evaluating $\% 2$ hydroelectric $\% 20$ project $\% 20$ effects $\% 20-\%$ 20Stillwater\%20-\%202006.pdf

Takeuchi, K., Moreau, D. H., 1974. "Optimal control of multiunit interbasin water resource systems". Water Resources Research 10 (3), 407-414.

Tang, B., 1993. "Orthogonal array-based Latin hypercubes". Journal of the American Statistical Association 88 (424), 1392-1397.

Teixeira, A. S., Mariño, M. A., 2002. "Coupled reservoir operation-irrigation scheduling by dynamic programming". Journal of Irrigation and Drainage Engineering 128 (2), 63-73.

Terry, L. A., Pereira, M. V. F., Neto, T. A. A., Silva, L. F. C. A., Sales, P. R. H., 1986. "Coordinating the energy generation of the Brazilian national hydrothermal electrical generating system". Interfaces 16 (1), 16-38.

Torcellini, P. A., Long, N., Judkoff, R. D., 2004. "Consumptive water use for US power production.". ASHRAE Transactions 110 (1), 96-100.

Trussart, S., Messier, D., Roquet, V., Aki, S., 2002. "Hydropower projects: a review of most effective mitigation measures". Energy Policy 30 (14), 1251-1259.

Turgeon, A., 1980. "Optimal operation of multireservoir power systems with stochastic inflows". Water Resources Research 16 (2), 275-283.

Turgeon, A., 1982. "Incremental dynamic programing may yield nonoptimal solutions". Water Resources Research 18 (6), 1599-1604. 
UN, 1992. "Agenda 21: programme of action for sustainable development". Tech. rep., United Nations.

URL http://sustainabledevelopment.un.org/content/documents/Agenda21.pdf

University of Colorado, 2014. RiverWare. Technical documentation version 6.5. Optimization. University of Colorado, Boulder, USA.

Uritskaya, O. Y., Uritsky, V. M., 2015. "Predictability of price movements in deregulated electricity markets". Energy Economics 49, 72-81.

USBR, 1976. "Selecting hydraulic reaction turbines. A water resources technical publication engineering monograph no. 20". Tech. rep., United States Department of the Interior, Bureau of Reclamation.

URL http://www.usbr.gov/pmts/hydraulics_lab/pubs/EM/EM20.pdf

Vargas, L. S., Quintana, V. H., Vannelli, A., 1993. "A tutorial description of an interior point method and its applications to security-constrained economic dispatch". IEEE Transactions on Power Systems 8 (3), 1315-1324.

Ventura, E. S., Martinez, L., 28 August - 2 September 2011. "A comparative study of closedloop control policy for long term hydrothermal scheduling". In: World congress. IFAC, Milano, Italy.

Veselka, T. D., Portante, E. C., Koritarov, V., Hamilton, S., VanKuiken, J. C., McCoy, J. J., Paprockas, K. R., North, M. J., Kavicky, J. A., Guziel, K. A., Moulton, R., Poch, L. A., Folga, S. M., Tompkins, M. M., Sedlacek, J., Novickas, A. A., 1995. "Impacts of Western Area Power Administration's power marketing alternatives on electric utility systems". Tech. rep., Argonne National Laboratory, University of Chicago.

URL http://inis.iaea.org/search/search .aspx?orig_q=RN : 27052137

von Neumann, J., Morgenstern, O., 1944. Theory of games and economic behavior. Princeton University Press, Princeton, USA.

Wallace, R., Bassuk, E., 1991. "Housing famine and homelessness: how the low-income housing crisis affects families with inadequate supports". Environment and Planning A 23 (4), $485-498$.

Wallace, S. W., Fleten, S.-E., 2003. Handbooks in operations research and management science. Vol. 10. Elsevier, Amsterdam, Holland, Ch. 10, pp. 637-677.

Wang, J., Zhang, Y., 2012. "Short-term optimal operation of hydropower reservoirs with unit commitment and navigation". Journal of Water Resources Planning and Management $138(1), 3-12$. 
Warland, G., Haugstad, A., Huse, E. S., 14-18 July 2008. "Including thermal unit start-up costs in a long-term hydro-thermal scheduling model". In: 16th power systems computation conference. PSCC, Glasgow, Scotland.

Warnaka, K., Pochop, L., 1988. "Analyses of equations for free water evaporation estimates". Water Resources Research 24 (7), 979-984.

Warnick, C. C., 1984. Hydropower engineering. Prentice-Hall Incorporated, Englewood Cliffs, USA.

Weedy, B. M., 1972. Electric power systems. John Wiley \& Sons Ltd, London, England.

Wells, W. C., 1818. Two essays: one upon single vision with two eyes; the other on dew. A letter to the Right Hon. LLoyd, Lord Kenyon and an account of a female of the white race of mankind, part of whose skin resembles that of a negro;... by the late William Charles Wells,... with a memoir of his life, written by himself. Archibald Constable and Company, London, England.

Wilks, D. S., 2006. Statistical methods in the atmospheric sciences, 2nd Edition. Vol. 91 of International Geophysics Series. Elsevier, Burlington, USA.

Winter, T. C., Rosenberry, D. O., Sturrock, A. M., 1995. "Evaluation of 11 equations for determining evaporation for a small lake in the north central United States". Water Resources Research 31 (4), 983-993.

World Water Council, 2003. "The 3rd World Water Forum final report". Tech. rep., Secretariat of the 3rd World Water Forum.

URL http://www.worldwatercouncil.org/fileadmin/world_water_council/ documents/world_water_forum_3/3d_World_Water_Forum_FinalReport_BD.pdf

Wurbs, R. A., 1993. "Reservoir-system simulation and optimization models". Journal of Water Resources Planning and Management 119 (4), 455-472.

Xu, C. Y., Singh, V. P., 1998. "Dependence of evaporation on meteorological variables at different time-scales and intercomparison of estimation methods". Hydrological Processes $12(3), 429-442$.

Xu, C. Y., Singh, V. P., 2001. "Evaluation and generalization of temperature-based methods for calculating evaporation". Hydrological Processes 15 (2), 305-319.

Yakowitz, S., 1982. "Dynamic programming applications in water resources". Water Resources Research 18 (4), 673-696.

Yeh, W. W.-G., 1985. "Reservoir management and operations models: a state-of-the-art review". Water Resources Research 21 (12), 1797-1818. 
Yeh, W. W.-G., 16-20 May 2010. "Optimization of hydrosystem operation - A 40-year retrospective". In: World environmental and water resources congress 2010: challenges of change. ASCE, Providence, USA.

Yeh, W. W.-G., Chu, W.-S., Becker, L., 1979. "Real-time hourly reservoir operation". Journal of the Water Resources Planning and Management Division 105 (2), 187-203.

Yi, J., Labadie, J. W., Stitt, S., 2003. "Dynamic optimal unit commitment and loading in hydropower systems". Journal of Water Resources Planning and Management 129 (5), 388-398.

Yu, Z., Sparrow, F. T., Bowen, B. H., 1998. "A new long-term hydro production scheduling method for maximizing the profit of hydroelectric systems". Power Systems, IEEE Transactions on 13 (1), 66-71.

Zagona, E. A., Fulp, T. J., Shane, R., Magee, T., Goranflo, H. M., 2001. "Riverware: a generalized tool for complex reservoir system modelling". Journal of the American Water Resources Association 37 (4), 913-929.

Zambelli, M., Siqueira, T. G., Cicogna, M., Soares, S., 18-22 June 2006. "Deterministic versus stochastic models for long term hydrothermal scheduling". In: Power engineering society general meeting. IEEE, Montreal, Canada.

Zhang, M., Wei, X., Sun, P., Liu, S., 2012. "The effect of forest harvesting and climatic variability on runoff in a large watershed: the case study in the upper Minjiang River of Yangtze River basin". Journal of Hydrology 464 (1), 1-11.

Zhao, Y., Chen, X., Jia, Q.-S., Guan, X., Zhang, S., Jiang, Y., 2010. "Long-term scheduling for cascaded hydro energy systems with annual water consumption and release constraints". IEEE Transactions on Automation Science and Engineering 7 (4), 969-976. 


\section{Nomenclature}

\section{Abbreviations}

$\varphi$

p

F

V

\section{Acronyms}

DP

LP

Constants

$K_{c 1}$

$K_{c 2}$

\section{Indexes}

$a$

$b$

$c, \mathrm{c}$

$d$

ff

fv

if

$i v$

j

$k$

Minimum flows [\%].

Maximum ramping rates $[\mathrm{h}]$.

Flow released by the reservoir.

Water volume stored in the reservoir.

Dynamic programming.

Linear programming.

Conversion factor $\left[0.0036 \mathrm{hm}^{3} /\left(\mathrm{m}^{3} / \mathrm{s}\right)\right]$.

Conversion factor $\left[0.6048^{-1} h m^{3} /\left(\mathrm{m}^{3} / \mathrm{s}\right)\right]$.

Water inflow of the subproblem.

Average energy price of the subproblem.

Curve of the set of performance curves.

Volume segment of the reservoir storage-maximum technical plant flow.

Final $\mathrm{F}$ of the subproblem.

Final V of the subproblem.

Initial $\mathrm{F}$ of the subproblem.

Initial $\mathrm{V}$ of the subproblem.

Volume segment of the reservoir storage-water value curve.

Problem stage. 


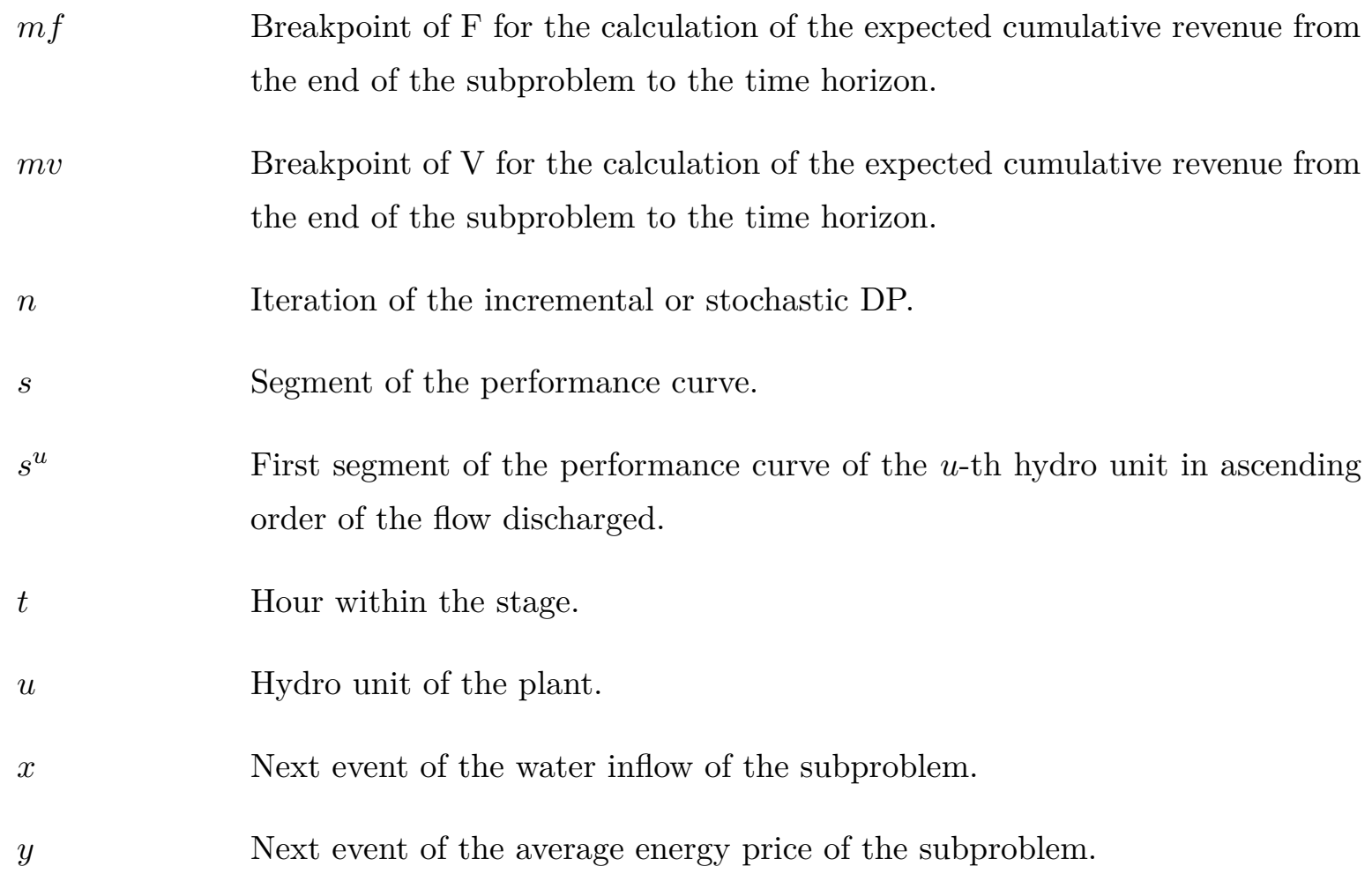

\section{Parameters}

Wear and tear costs of hydro units due to variations in the generated power $[€ / M W]$.

Shut-down and start-up costs for hydro units [€].

Virtual cost, neglected during the recalculations, of the bottom outlets and the spillways due to variations in the released flow $\left[€ /\left(\mathrm{m}^{3} / \mathrm{s}\right)\right]$.

$\rho^{\max } \quad$ Maximum value of $\rho$ to evaluate $\left[\left(\mathrm{m}^{3} / \mathrm{s}\right) / h\right]$.

$\rho_{k}^{\text {down }} \quad$ Down $\rho$ during the stage $k\left[\left(\mathrm{~m}^{3} / \mathrm{s}\right) / h\right]$.

$\rho_{k}^{u p} \quad$ Up $\rho$ during the stage $k\left[\left(\mathrm{~m}^{3} / \mathrm{s}\right) / h\right]$.

$\varphi^{\max } \quad$ Maximum value of $\varphi$ to evaluate $\left[\mathrm{m}^{3} / \mathrm{s}\right]$.

$\varphi_{k} \quad \varphi$ at the stage $k\left[\mathrm{~m}^{3} / \mathrm{s}\right]$.

$c^{\max } \quad$ Total number of performance curves of the subproblem.

$\overline{c^{\max }} \quad$ Maximum number of performance curves of the subproblem.

$E_{k} \quad$ Rate of hourly evaporated water volume per flooded area during the stage $k$ $\left[h \mathrm{~m}^{3} / \mathrm{km}^{2}\right]$.

$\bar{f}^{m f} \quad \mathrm{~F}$ at the $m f$-th breakpoint for the calculation of the expected cumulative revenue from the end of the subproblem to the time horizon $\left[\mathrm{m}^{3} / \mathrm{s}\right]$. 
$F_{0} \quad \mathrm{~F}$ at the beginning of the planning period $\left[\mathrm{m}^{3} / \mathrm{s}\right]$

$g^{\max } \quad$ Maximum hydropower plant power output of the subproblem $[M W]$.

$g^{\min } \quad$ Minimum hydropower plant power output according to the performance curve $[M W]$.

$g^{m i n, \varsigma} \quad$ Minimum hydropower plant power output according to the ç-th performance curve $[M W]$.

$H^{\%} \quad$ Percentage of the range of operating gross head that must be "covered" by each performance curve.

$H_{k}^{\max } \quad$ Maximum normal operating gross head during the stage $k[m]$.

$H_{k}^{\min } \quad$ Minimum normal operating gross head during the stage $k[m]$.

$j^{\max }$

Total number of segments of the reservoir storage-water value curve.

$k^{\max }$

Final stage of the problem.

$K_{b o 1}, K_{b o 2} \quad$ Coefficients of the linear approximation of the reservoir storage-maximum bottom outlet flow curve $\left[\left(\mathrm{m}^{3} / \mathrm{s}\right) / \mathrm{hm^{3 }} ; \mathrm{m}^{3} / \mathrm{s}\right]$.

$K_{e 1}, K_{e 2} \quad$ Coefficients of the linear approximation of the reservoir storage-flooded area curve $\left[\mathrm{km}^{2} / \mathrm{hm} \mathrm{m}^{3} ; \mathrm{hm}^{3}\right]$.

$K_{s p} \quad$ Coefficient of the linear approximation of the reservoir storage-maximum spillway flow curve $\left[\left(\mathrm{m}^{3} / \mathrm{s}\right) / \mathrm{hm}^{3}\right]$.

$m f^{\max } \quad$ Total number of breakpoints of $\mathrm{F}$ for the calculation of the expected cumulative revenue from the end of the subproblem to the time horizon.

$m v^{\max } \quad$ Total number of breakpoints of $\mathrm{V}$ for the calculation of the expected cumulative revenue from the end of the subproblem to the time horizon.

$p_{t}$

Energy price during the hour $t[€ / M W]$.

$P_{k} \quad$ Mean of the energy prices during the stage $k[€ / M W]$.

$q^{\max } \quad$ Maximum plant flow during subproblem $\left[\mathrm{m}^{3} / \mathrm{s}\right]$.

$q^{\max , s} \quad$ Maximum flow of the $s$-th segment of the performance curves $\left[\mathrm{m}^{3} / \mathrm{s}\right]$.

$q^{u} \quad$ Plant flow above which the $u$-th hydro unit starts up $\left[\mathrm{m}^{3} / \mathrm{s}\right]$.

$Q^{\max } \quad$ Maximum technical plant flow $\left[\mathrm{m}^{3} / \mathrm{s}\right]$.

$Q^{\max , d} \quad$ Plant flow corresponding to the $d$-th volume segment of the reservoir storagemaximum technical plant flow $\left[\mathrm{m}^{3} / \mathrm{s}\right]$. 
$Q^{\text {maxbo }} \quad$ Maximum flow through the bottom outlets $\left[\mathrm{m}^{3} / \mathrm{s}\right]$.

$Q^{\min } \quad$ Minimum technical plant flow $\left[\mathrm{m}^{3} / \mathrm{s}\right]$.

$r^{s}$

$r^{s, \varsigma}$

$s^{\max }$

Slope of the $s$-th segment of the performance curve $\left[M W /\left(\mathrm{m}^{3} / s\right)\right]$.

Slope of the $s$-th segment of the ç-th performance curve $\left[M W /\left(m^{3} / s\right)\right]$.

Total number of segments of the performance curves.

$t^{\max }$

Total number of hours per stage.

$u^{\max }$

Total number of hydro units of the plant.

$v^{c}$

$\mathrm{V}$ of the corresponding one to the $c$-th performance curve $\left[\mathrm{hm}^{3}\right]$.

$\bar{v}^{m v}$

$V^{\#}$

$\mathrm{V}$ at the $m v$-th breakpoint for the calculation of the expected cumulative revenue from the end of the subproblem to the time horizon $\left[\mathrm{hm}^{3}\right]$.

$V^{\text {dead }} \quad$ V below the bottom outlets $\left[\mathrm{hm}^{3}\right]$.

$\mathrm{V}$ at the beginning and end of the planning period $\left[\mathrm{hm}^{3}\right]$.

$V_{0} \quad \mathrm{~V}$ at the beginning of the planning period $\left[\mathrm{hm}^{3}\right]$.

$V_{k}^{\text {maxleg }} \quad$ Maximum legal $\mathrm{V}$ during the stage $k\left[\mathrm{hm}^{3}\right]$.

$V^{\text {maxtec }} \quad$ Maximum technical $\mathrm{V}$ above which it is not convenient or possible to generate power $\left[\mathrm{hm}^{3}\right]$.

$V^{\text {mintec }} \quad$ Minimum technical V below which it is not convenient or possible to generate power $\left[\mathrm{hm}^{3}\right]$.

$V^{s p} \quad$ V above which the spillways can operate $\left[\mathrm{hm}^{3}\right]$.

$V Q^{d} \quad$ Minimum $\mathrm{V}$ of the $d$-th segment of the reservoir storage-maximum technical plant flow $\left[\mathrm{hm}^{3}\right]$.

$V W V^{j} \quad$ V of the $j$-th segment of the reservoir storage-water value curve $\left[\mathrm{hm}^{3}\right]$.

$w_{t} \quad$ Water inflow to the reservoir during the hour $t\left[\mathrm{~m}^{3} / \mathrm{s}\right]$.

$W^{\text {avg }} \quad$ Average river flow during the year $\left[\mathrm{m}^{3} / \mathrm{s}\right]$.

$W V_{k+1}^{j}{ }^{a, b} \quad$ Water value of the $j$-th segment of the reservoir storage-water value curve at the beginning of the stage $k+1$ weighted by the transition probabilities corresponding to $\left(W_{k}^{a}, P_{k}^{b}\right)[€ / M W]$.

$\bar{z}^{m v, m f, a, b} \quad$ Expected cumulative revenue at $\left(V^{m v}, F^{m f}, W_{k}^{a}, P_{k}^{b}\right)$ from the stage $k$ to the time horizon $[€]$. 


\section{Sets}

$\mathbb{C}_{k}^{i v, f v} \quad$ Performance curves of the subproblem corresponding to the decision to go from $V_{k}^{i v}$ to $V_{k+1}^{f v}$.

$\mathbb{D}$

$\mathbb{J}$

$\mathbb{K}$

$\mathbb{M}^{F}$

$\mathbb{M}^{V}$

$\mathbb{S}$

$\mathbb{T}$

$\mathbb{U}$

$\mathbb{V}_{k}$

$\mathbb{V} \mathbb{F}_{k}$

$\mathbb{V} \mathbb{F} \mathbb{W} \mathbb{P}_{k}$

$\mathbb{V} \mathbb{W} \mathbb{P}_{k}$

Volume segments of the reservoir storage-maximum technical plant flow.

Segments of the reservoir storage-water value curve.

Stages of the problem.

Breakpoints of $\mathrm{F}$ for the calculation of the expected cumulative revenue from the end of the subproblem to the time horizon.

Breakpoints of $\mathrm{V}$ for the calculation of the expected cumulative revenue from the end of the subproblem to the time horizon.

Segments of the performance curves.

Hours of the stage.

Hydro units of the plant.

Feasible states within the discretisation of $\mathrm{V}$ during the stage $k$.

Feasible states within the discretisation of $\mathrm{V}$ and $\mathrm{F}$ during the stage $k$.

Feasible states within the discretisation of V, F, water inflow and energy price during the stage $k$.

Feasible states within the discretisation of V, water inflow and energy price during the stage $k$.

\section{Binary variables}

$b q^{m f} \quad=1$ if $\mathrm{F}$ at the hour $t^{\max }$ is within the interval $[m f, m f+1]$ for the calculation of the expected cumulative revenue from the end of the subproblem to the time horizon.

$b v^{m v} \quad=1$ if $\mathrm{V}$ at the hour $t^{m a x}$ is within the interval $[m v, m v+1]$ for the calculation of the expected cumulative revenue from the end of the subproblem to the time horizon.

$o l_{t}^{u} \quad=1$ if the $u$-th hydro unit is on-line during the hour $t$.

$p c_{t}^{c} \quad=1$ if $\mathrm{V}$ is above $v^{c}$ during the hour $t$.

$s d_{t}^{u} \quad=1$ if the $u$-th hydro unit is shut-down during the hour $t$.

$s p_{t} \quad=1$ if $\mathrm{V}$ is above $V^{s p}$ during the hour $t$. 
$s u_{t}^{u} \quad=1$ if the $u$-th hydro unit is started-up during the hour $t$.

$v q_{t}^{d} \quad=1$ if $\mathrm{V}$ is above $V Q^{d}$ during the hour $t$.

$w v^{j} \quad=1$ if $\mathrm{V}$ is above the minimum $\mathrm{V}$ of the $j$-th segment of the reservoir storagewater value curve.

\section{Non-negative variables}

$\varepsilon$

$\lambda_{t}$

$\mu_{t}$

$\theta_{k}^{a, x}$

$\theta_{k}^{b, y}$

$c q^{m f}$

$d_{t}$

$e_{t}$

$f_{t^{m a x}}^{d e c}$

$f_{t^{\max }}^{\text {inc }}$

$F_{k}^{f i n}$

$f_{t}$

$F_{k}^{i f}$

$F_{k+1}^{f f}$

$g_{t}$

$g_{t}^{d e c}$

$g_{t}^{i n c}$

$g_{t}^{v_{t}, f_{t}}$
Long-term economic impact caused by $\varphi$ and/or $\rho[\%]$.

Lagrange multiplier of an equality constraint during the hour $t[€]$.

Lagrange multiplier of an inequality constraint during the hour $t[€]$.

Probability that the water inflow at stage $k+1$ is $x$ given that at stage $k$ it was $a$.

Probability that the average energy price at stage $k+1$ is $y$ given that at stage $k$ it was $b$.

Variable whose maximum value is 1 corresponding to the $m f$-th breakpoint of $\mathrm{F}$ for the calculation of the expected cumulative revenue from the end of the subproblem to the time horizon.

Decision which corresponds to the hourly variation of $f_{t}$ during the hour $t$ $\left[\mathrm{m}^{3} / \mathrm{s}\right]$.

Evaporation flow during the hour $t\left[\mathrm{~m}^{3} / \mathrm{s}\right]$.

Decrease in flow released by the reservoir respect to the value obtained in the estimation step during hour $t^{\max }\left[\mathrm{m}^{3} / \mathrm{s}\right]$.

Increase in flow released by the reservoir respect to the value obtained in the estimation step during hour $t^{\max }\left[\mathrm{m}^{3} / \mathrm{s}\right]$.

F at the end of the stage $k\left[\mathrm{~m}^{3} / \mathrm{s}\right]$.

Flow released by the reservoir $\left[\mathrm{m}^{3} / \mathrm{s}\right]$.

F corresponding to the state if at the beginning of the stage $k\left[\mathrm{~m}^{3} / \mathrm{s}\right]$.

F corresponding to the state $f f$ at the beginning of the stage $k+1\left[\mathrm{~m}^{3} / \mathrm{s}\right]$.

Generated power during the hour $t[M W]$.

Decrease in generated power between the hours $t$ and $t+1[M W]$.

Increase in generated power between the hours $t$ and $t+1[M W]$.

Power generated by releasing $f_{t}$ at $v_{t}$ during the hour $t[M W]$. 
$G_{k}^{i v, f v} \quad$ Generated power corresponding to the decision to go from $V_{k}^{i v}$ to $V_{k+1}^{f v}$ linearly interpolated in the generation characteristic from the weekly average values of both the net head and the plant operating flow $[M W]$.

$G^{\text {max }, i v, v_{k}^{\min f, i v, a, b}}$ Average of the maximum generated powers corresponding to go from $V_{k}^{i v}$ to $v_{k}^{\min f, i v, a, b}[M W]$.

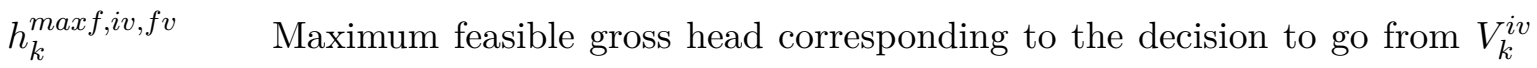
to $V_{k+1}^{f v}[m]$.

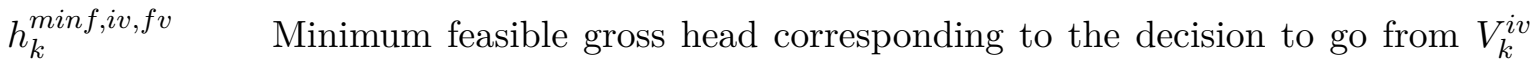
to $V_{k+1}^{f v}[m]$.

$H_{k}^{i v, f v} \quad$ Mean gross head of the reservoir corresponding to the decision to go from $V_{k}^{i v}$ to $V_{k+1}^{f v}[m]$.

$P_{k}^{b} \quad$ Average energy price of the stage $k$ corresponding to the state $b[€ / M W]$.

$q_{t} \quad$ Plant flow during the hour $t\left[\mathrm{~m}^{3} / \mathrm{s}\right]$.

$q_{t}^{b o} \quad$ Flow released through the bottom outlets during the hour $t\left[\mathrm{~m}^{3} / \mathrm{s}\right]$.

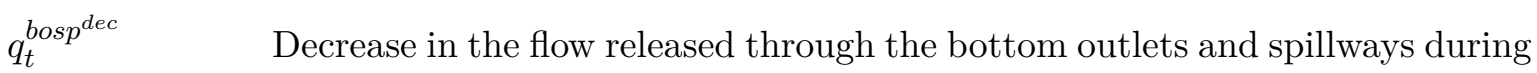
the hour $t\left[\mathrm{~m}^{3} / \mathrm{s}\right]$.

$q_{t}^{\text {bosp }}$ inc $\quad$ Increase in the flow released through the bottom outlets and spillways during the hour $t\left[\mathrm{~m}^{3} / \mathrm{s}\right]$.

$q_{1, k}^{o s} \quad$ Plant flow at the beginning of the stage $k$, according to the optimal solution obtained by $\bmod 3 V s t u 1$ or by $\bmod 3 V s t u 2\left[\mathrm{~m}^{3} / \mathrm{s}\right]$.

$q_{t^{\max }, k}^{o s} \quad$ Plant flow at the end of the stage $k$, according to the optimal solution obtained by $\bmod 3 V s t u 1$ or by $\bmod 3 V s t u 2\left[\mathrm{~m}^{3} / \mathrm{s}\right]$.

$q_{t}^{s} \quad$ Plant flow corresponding to the $s$-th segment of the performance curves during the hour $t\left[\mathrm{~m}^{3} / \mathrm{s}\right]$.

$q_{t}^{s p} \quad$ Flow released through the spillways during the hour $t\left[\mathrm{~m}^{3} / \mathrm{s}\right]$.

$T_{k}^{o p e, i v, f v} \quad$ Number of operating hours corresponding to the decision to go from $V_{k}^{i v}$ to $V_{k+1}^{f v}$.

$v_{t} \quad \mathrm{~V}$ at the end of the hour $t\left[\mathrm{hm}^{3}\right]$.

$v_{t}^{a b o s p} \quad \mathrm{~V}$ at the end of the hour $t$ above the spillways $\left[\mathrm{hm}^{3}\right]$.

$v_{t}^{\text {belsp }} \quad \mathrm{V}$ at the end of the hour $t$ below the spillways $\left[\mathrm{hm}^{3}\right]$. 
$v_{t^{m a x}}^{d e c} \quad$ Decrease in $\mathrm{V}$ of the reservoir respect to the value obtained by mod2stu3 during hour $t^{\max }\left[\mathrm{hm}^{3}\right]$.

$v_{t^{\max }}^{\text {inc }} \quad$ Increase in $\mathrm{V}$ of the reservoir respect to the value obtained by mod2stu3 during hour $t^{\max }\left[\mathrm{hm}^{3}\right]$.

$v_{k}^{\max f, i v, a, b} \quad$ Maximum feasible $\mathrm{V}$ from $\left(V_{k}^{i v}, W_{k}^{a}, P_{k}^{b}\right)$ at the end of the stage $k\left[\mathrm{hm}^{3}\right]$.

$v_{k}^{\max f, i v, f v} \quad$ Maximum feasible $\mathrm{V}$ corresponding to the decision to go from $V_{k}^{i v}$ to $V_{k+1}^{f v}$ $\left[h \mathrm{~m}^{3}\right]$.

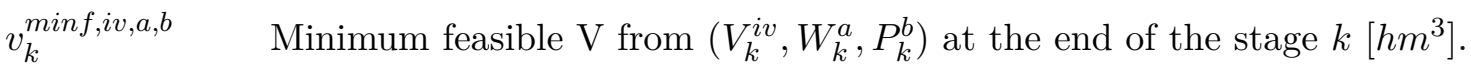

$V_{k}^{f i n} \quad \mathrm{~V}$ at the end of the stage $k\left[\mathrm{hm}^{3}\right]$.

$V_{k+1}^{f v} \quad \mathrm{~V}$ corresponding to the state $f v$ at the beginning of stage $k+1\left[\mathrm{hm}^{3}\right]$.

$V_{k}^{i v} \quad \mathrm{~V}$ corresponding to the state $i v$ at the beginning of the stage $k\left[\mathrm{hm}^{3}\right]$.

$V_{k}^{r e l, i v, f v} \quad$ Total released water volume corresponding to the decision to go from $V_{k}^{i v}$ to $V_{k+1}^{f v}\left[h m^{3}\right]$.

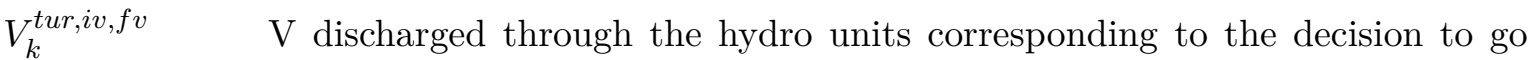
from $V_{k}^{i v}$ to $V_{k+1}^{f v}\left[h m^{3}\right]$.

$v w v^{j} \quad \mathrm{~V}$ in the $j$-th segment of the reservoir storage-water value curve $\left[\mathrm{hm}^{3}\right]$.

$W_{k}^{a} \quad$ Water inflow of the stage $k$ corresponding to the state $a\left[\mathrm{hm}^{3}\right]$.

$z_{k}^{i v, f v} \quad$ Revenue corresponding to the decision to go from $V_{k}^{i v}$ to $V_{k+1}^{f v}[€]$.

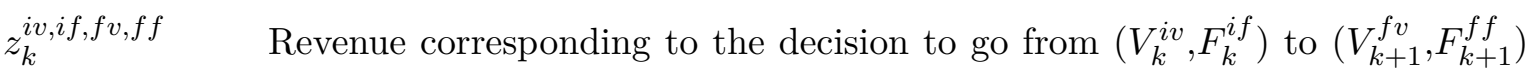
$[€]$.

$z_{k}^{i v, a, b, V_{k}^{f i n}} \quad$ Revenue corresponding to the decision to go from $\left(V_{k}^{i v}, W_{k}^{a}, P_{k}^{b}\right)$ to $V_{k}^{f i n}[€]$. $z_{k}^{i v, i f, a, b, V_{k}^{f i n}, F_{k}^{f i n}}$ Revenue corresponding to the decision to go from $\left(V_{k}^{i v}, F_{k}^{i f}, W_{k}^{a}, P_{k}^{b}\right)$ to $\left(V_{k}^{f i n}, F_{k}^{f i n}\right)[€]$.

$\bar{z}^{v_{t} \max } \quad$ Future expected revenue starting from $v_{t}^{\max }$ at the end of the planning period $[€]$.

$\bar{z}^{v_{t} \max , f_{t} \max } \quad$ Future expected revenue starting from $v_{t}^{\max }$ and $f_{t^{\max }}$ at the end of the planning period $[€]$.

$Z_{k}^{i v} \quad$ Optimum cumulative revenue at $V_{k}^{i v}$ from the stage $k$ to the time horizon $[€]$.

$Z_{k}^{i v, i f} \quad$ Optimum cumulative revenue at $\left(V_{k}^{i v}, F_{k}^{i f}\right)$ from the stage $k$ to the time horizon $[€]$. 
$Z)^{n} \quad$ Optimum cumulative revenue at the iteration $n$ of the incremental DP [€].

$\bar{Z}_{k}^{i v, a, b} \quad$ Optimum cumulative expected revenue at $\left(V_{k}^{i v}, W_{k}^{a}, P_{k}^{b}\right)$ from the stage $k$ to the time horizon $[€]$.

$\left.\bar{Z}_{k}^{i v, a, b}\right)^{n} \quad$ Optimum cumulative expected revenue at $\left(V_{k}^{i v}, W_{k}^{a}, P_{k}^{b}\right)$ from the stage $k$ to the time horizon of the iteration $n$ of the stochastic DP [€].

$\bar{Z}_{k}^{i v, i f, a, b} \quad$ Optimum cumulative expected revenue at $\left(V_{k}^{i v}, F_{k}^{i f}, W_{k}^{a}, P_{k}^{b}\right)$ from the stage $k$ to the time horizon $[€]$.

$\left.\bar{Z}_{k}^{i v, i f, a, b}\right)^{n} \quad$ Optimum cumulative expected revenue at $\left(V_{k}^{i v}, F_{k}^{i f}, W_{k}^{a}, P_{k}^{b}\right)$ from the stage $k$ to the time horizon of the iteration $n$ of the stochastic DP [€].

$\bar{Z}_{k+1}^{V_{k}^{f i n}, x, y} \quad$ Optimum cumulative expected revenue at $\left(V_{k}^{f i n}, W_{k+1}^{x}, P_{k+1}^{y}\right)$ from the stage $k+1$ to the time horizon $[€]$.

$\bar{Z}_{k+1}^{V_{k}^{f i n}, F_{k}^{f i n}, x, y}$ Optimum cumulative expected revenue at $\left(V_{k}^{f i n}, F_{k}^{f i n}, W_{k+1}^{x}, P_{k+1}^{y}\right)$ from the stage $k+1$ to the time horizon [€].

$\bar{Z}_{k+1}^{V_{k}^{f i n}(a, b} \quad$ Optimum cumulative expected revenue at $V_{k}^{f i n}$ from the stage $k+1$ to the time horizon weighted by the transition probabilities corresponding to $\left(W_{k}^{a}, P_{k}^{b}\right)[€]$.

$\bar{Z}_{k+1}^{V_{k}^{f i n}, F_{k}^{f i n}}\left({ }^{a, b}\right.$ Optimum cumulative expected revenue at $\left(V_{k}^{f i n}, F_{k}^{f i n}\right)$ from the stage $k+1$ to the time horizon weighted by the transition probabilities corresponding to $\left(W_{k}^{a}, P_{k}^{b}\right)[€]$. 